\title{
PARTICLE DISPLACEMENT AND VELOCITY MEASUREMENT USING HOLOGRAPHY
}

\author{
by \\ Phillip Herbert Malyak \\ Submitted in Partial Fulfillment \\ of the \\ Requirements for the Degree \\ DOCTOR OF PHILOSOPHY
}

Supervised by Provost Brian J. Thompson

The Institute of Optics

College of Engineering and Applied Science

University of Rochester

Rochester, New York 


\section{Curriculum Vitae}

The author was born in Lackawanna, New York on April 18, 1956. Following graduation from Lackawanna Senior High School in 1974, he attended The State University of New York at Buffalo and recieved the B.S. degree in Electrical Engineering in 1978 and the M.S. degree in Electrical Engineering in 1980.

The author began graduate studies at The Institute of Optics in September of 1980. His studies were supported by a Departmental Fellowship for the 1980-81 academic year, by a Research Assistantship for the years 1981 to 1984 and by a Link Foundation Engineering Fellowship for the 1984-85 academic year. The author's research at The Institute of Optics was supervised by Provost Brian J. Thompson . 


\section{Acknowledgements}

I wish to extend sincere thanks and appreciation to my advisor, Provost Brian J. Thompson. The insight and direction he provided during the course of this research was invaluable. In addition, his support and enthusiasm provided the inspiration that I needed from time to time.

I am grateful to faculty members and students at The Institute of Optics who have provided technical and material support throughout the course of this research. In particular, I would like to thank Prof. J.C. Dainty for allowing me to use his image digitizer and computing facilities. Dr. Bryan Brames was most helpful in familiarizing me to this system. I wish to extend thanks to Prof. G.M. Morris for giving me access to his image digitizing equipment following Prof. Dainty's move to Imperial College. It is also a pleasure to acknowledge helpful discussions with Dr. John Bortz, Dr. Bryan Brames, Dr. Joe Crane and Dr. Paul Dunn and Mr. Joseph Marron.

I am indebted to several individuals who have provided much needed emotional support over the past few years, especially my parents, my brother, Ms. Donna Winderl, Ms. Beverly Hecht, Mr. Frank Gerard and Mr. Steven Greene. I thank them all.

Finally, it is pleasure to acknowledge the generous support provided by The Link Foundation, NASA, and the Rochester Gas and Electric Corporation Energy Fund. 


\begin{abstract}
A method of measuring particle displacements and velocities in the transform plane of a double-exposure hologram is examined both theoretically and experimentally. The analysis begins with a study of the transform patterns obtained from single-exposure holograms (both in-line holograms and off-axis holograms are considered). Although the transform of an in-line hologram and an off-axis hologram are similar, the on-axis images of an in-line hologram are shown to produce a zone lens which is not observed in the transform plane of an off-axis hologram. The Fourier transform patterns of double-exposure holograms are then examined, and it is shown that each pair of particle images related by translation contributes a set of cosine fringes to the total diffraction pattern. The frequency and orientation of the fringes are related to the magnitude and direction, respectively, of a given particle's displacement or velocity. The transform patterns corresponding to 1- and 2-dimensional distributions of displacement are examined in detail. For the 1-dimensional case, a theoretical analysis indicates that the probability density function that characterizes the distribution of displacements may be determined by properly processing intensity data measured in the transform plane of a doubly exposed hologram. For the 2-dimensional case it is shown that the marginal density functions may be obtained if the orthogonal components of particle displacement are statistically
\end{abstract}


independent. Experimental results are presented, and good agreement between theory and experiment is observed. 
Table of Contents

$\begin{array}{lr} & \text { Page } \\ \text { CURRICULUM VITAE } & \text { i i } \\ \text { ACKNOWLEDGEMENTS } & \text { ii } \\ \text { ABSTRACT } & \text { iv } \\ \text { LIST OF FIGURES } & \mathbf{x} \\ \text { CHAPTER } & \end{array}$

I. BACKGROUND AND INTRODUCTORY REMARKS 1

1.1 Introduction 1

1.1.1 In-1ine far-field holography 2

1.1.2 Off-axis holography 9

1.2 Particle velocity measurement using holography 12

1.2.1 Applications 14

1.2.2 Automated readout 16

1.2.3 Digital vs. optical-digital processing
of data from particle field holograms

1.3 Other optical techniques for measuring particle velocities

1.3.1 Laser-Doppler velocimetry 21

1.3.2 Laser speckle photography 24

1.4 Thesis content $\quad 25$

II. FOURIER TRANSFORMS OF RECONSTRUCTED IMAGES FROM PARTICLE HOLOGRAMS 27

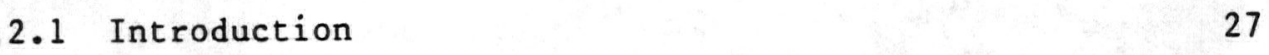


2.2 The Fourier transform of a singly exposed inline hologram

2.2.1 The transform of a hologram of a single particle

2.2.2 The transform of a hologram of many particles - case 1: Particles confined to a plane parallel to the hologram plane

2.2.3 Some aspects of speckle in the transform plane of a particle field hologram

2.2.4 The transform of a hologram of many particles - case 2: Particles distributed throughout a volume

2.2.5 The effects of random particle orientations and particle size distributions in the transform plane

2.3 The Fourier transform of the reconstructed image of a singly exposed off-axis hologram

2.3.1 The off-axis hologram and the Fourier transform of the reconstrucrted images assuming 1 inear recording

2.3.2 The off-axis hologram assuming nonlinear recording

III. DOUBLY EXPOSED HOLOGRAMS: PARTICLE DISPLACEMENT MEASUREMENT IN THE FOURIER PLANE

3.1 Introduction

3.2 The Fourier transform of a doubly exposed inline far-field hologram

3.3 Illustrative examples: Some specific velocity distributions and the corresponding Fourier transform patterns

3.3.1 All particles have the same displacement between exposures 
3.3.2 The effect of a uniform distribution of particle displacements

3.3.3 The effect of a Gaussian distribution of particle displacements

3.3.4 The effect of a Gaussian distribution of displacements in two dimensions

3.3.5 The relationship between the width of the density function and the ability to measure it from data obtained in the transform plane of a double-exposure hologram

3.4 The use of double-exposure off-axis holography for particle displacement and velocity measurement

108

3.5 A method for obtaining one-dimensional displacement and velocity distributions from intensity measurements in the transform plane of a double-exposure hologram

3.6 The effects of a limited number of particles

3.7 Measuring two-dimensional velocity and displacement distributions in the transform plane of a double-exposure hologram

3.8 Measuring two-dimensional velocity and displacement distributions when the horizontal and vertical components of displacement are independent

3.9 Summary

138

IV. MEASURING PARTICLE DISPLACEMENTS IN THE TRANSFORM PLANE OF A DOUBLE-EXPOSURE HOLOGRAM: EXPERIMENTAL RESULTS

$\begin{array}{lll}4.1 & \text { Introduction } & 140\end{array}$

$\begin{array}{ll}\text { 4.2 Data acquisition } & 140\end{array}$

4.2.1 The data acquistion system in detail 142 
4.3 Recording holograms with known particle displacements

4.3.1 Recording a transparency of the random array of dots

4.4 Experimentally obtained measurements of onedimensional displacement and velocity distributions

4.4.1 Results obtained by using holograms of random arrays of opaque discs as input to the hybrid processing system

4.4.2 Measuring the velocty distribution of particles falling in water

170

4.5 Two-dimensional distributions: measuring the marginal density function

4.6 Limitations on the width of the velocity distribution

4.7 Summary

V. CONCLUSIONS AND SUGGESTIONS FOR FUTURE STUDY
APPENDIX A - The Effects of Intermodulation Terms in the Transform Plane of an In-line Far-Field Hologram

APPENDIX B - Multiple-Exposure Holography

APPENDIX C - Object Motion During Exposure 


\section{List of Figures}

Figure

Page

1.1 (a) Geometry for recording a far-field hologram. (b) Hologram reconstruction.

1.2 (a) Optical arrangement for laser-Doppler velocimeter. (b) Enlarged view of probe volume showing interference fringes.

2.1 (a) Hologram recording geometry. (b) Geometry for forming the optical Fourier transform.

2.2 The zone lens in the transform plane of an in-line hologram results from the interference of the spherical waves produced by the real and virtual images.

2.3 Optical Fourier transform of an in-line hologram of $50 \mu$ glass microspheres. The object-to-hologram separation is $6 \mathrm{~cm}(\mathrm{~N}=12)$ and $\lambda=5145 \AA$.

2.4 Optical Fourier transform of an in-line hologram of $50 \mu$ glass microspheres. The object-to-hologram separation is $12 \mathrm{~cm}(\mathrm{~N}=24)$ and $\lambda=5145 \AA$.

2.5 Total far-field diffraction pattern produced by $50 \mu$ glass microspheres.

2.6 Hologram recording geometry. The particles are assumed to be uniformly distributed within the interval $z_{1}<z<z_{2}$.

2.7 Illustrating the $z$-dependent structure in the transform plane of an in-line far-field hologram. (a) Plot of $\left\langle I_{F}(u, v)\right\rangle / I_{0}(u, v)$ vs. the radial coordinate $\rho$ for $\lambda=514.5 \mathrm{~nm}, f=0.5 \mathrm{~m}, z_{1}=4.0 \mathrm{~cm}$ and $z_{2}=7.8 \mathrm{~cm}$. (b) Plot of $\left\langle I_{F}(u, v)\right\rangle / I_{0}(u, v)$ vs. the radial coordinate $\rho$ for $\lambda=514.5 \mathrm{~nm}, f=0.5 \mathrm{~m}, z_{1}=z_{2}=5.9 \mathrm{~cm}$.

2.8 The optical Fourier transform of $52.8 \mu$ polysytrene spheres suspended in water. The physical depth of the sample was $5 \mathrm{~cm}$. 
2.9 Illustrating the apparent sample volume depth in an in-line hologram. The sample volume has length $z_{0}$ and index of refraction $n$; the air gap between the sample and the recording plane has length $z_{1}$. Applying Snell's law in paraxial form ( $n i=n^{\prime} r$ ) reveals that a particle located at a distance $z_{0}$ from the interface appears to be located at $z_{0} / n$.

2.10 Theoretical intensity profile in the Fourier transform plane of an in-line hologram of $52.8 \mu$ particles. The particles were assumed to be uniformly distributed along the axial interval, $4.0 \mathrm{~cm}<z<7.8 \mathrm{~cm}$.

2.11 Measured intensity profile resulting from a single scan along a radius in the optical Fourier transform plane of an in-line hologram of $52.8 \mu$ particles. The particles were distributed along the axial interval, $4.0 \mathrm{~cm}<z<7.8 \mathrm{~cm}$.

2.12 Same as Fig. 2.11, except 25 radial scans were averaged to reduce the fluctuations due to speckle.

2.13 (a) Recording geometry for off-axis holography. (b) Image reconstruction and Fourier transformation.

2.14 The intensity pattern in the transform plane of an off-axis hologram. $50 \mu$ glass microspheres were used in recording the hologram.

2.15 (a) Real images from an off-axis hologram of $50 \mu$ particles. (b) False images from a nonlinearly recorded off-axis hologram of $50 \mu$ particles.

2.16 Fourier transform pattern obtained from a nonlinearly recorded off-axis hologram of $50 \mu$ particles. A zone lens is formed in the Fourier plane as a result of the in-line false images.

3.1 (a) Optical Fourier transform pattern produced by a double-exposure far-field hologram of $80 \mu$ particles. The displacement between exposures was $0.3 \mathrm{~mm}$. (b) Same as (a) except $50 \mu$ particles were used and the displacement was $1 \mathrm{~mm}$. (c) A photograph of reconstructed images obtained from a double-exposure farfield hologram of $80 \mu$ particles.

3.2 Optical Fourier transform obtained from a doubleexposure off-axis hologram of $50 \mu$ particles. 
3.3 Plot of $\bar{F}\{a\}$ vs. $a$ (the transform variable). This plot illustrates the final step in obtaining the density function $p(\cdot)$ from the intensity data measured in the transform plane of a double-exposure far-field hologram.

3.4 (a) Plot of the theoretical intensity profile in the transform plane of a double-exposure hologram. (b) Magnitude of the FFT of the intensity profile vs. discrete transform variable $n$.

3.5 (a) Plot of $\left|\operatorname{FFT}\left\{I_{F}\right\}\right|$ vs. $n$ after low pass filtering. (b) The envelope function $I_{0}$ is obtained by forming the inverse FFT of the data in (a).

3.6 (a) Plot of $I_{F} / I_{0}$ vs. position. The fluctuations near $n=5$ and $n=120$ are due to the fact that $I_{F}$ was assumed to be obtained with an 8-bit digitizer (see accompanying text). (b) Plot of $\left|\mathrm{FFT}\left\{I_{F} / I_{0}\right\}\right|$ vs. discrete transform variable $n$.

119

3.7 (a) Same as Fig. 3.6a except the fluctuations near $n=5$ and $n=120$ were eliminated by setting $I_{F} / I_{0}$ to unity at these points. (b) Magnitude of the FFT of the curve in (a).

3.8 (a) Theoretical plot of $I_{F} / I_{0}$. (b) Magnitude of the FFT of the curve in (a).

3.9 (a) Plot of $I^{\prime}{ }_{N}$ vs. position for $N=100$. (b) $\left|\mathrm{FFT}\left\{I^{\prime} N\right\}\right|$ vs. $n$ (the transform variable).

3.10 (a) Plot of $I_{N}^{\prime}$ vs. position for $N=500$. (b) |FFT $\left\{I^{\prime}{ }_{N}\right\} \mid$ vs. $n$.

3.11 (a) Plot of $I^{\prime}{ }_{N}$ vs. position for $N=1000$. (b) | FFT $\left\{I^{\prime}{ }_{N}\right\} \mid$ vs. $n$.

3.12 (a) Two-dimensional density function obtained by a computer simulation assuming no stop is present in the optical transform plane. (b) The "density function" obtained when the stop is present.

3.13 Plot of an axial slice of the two-dimensional FFT of $I_{k l}^{\prime}$. The solid curve illustrates the result obtained in the ideal case assuming no stop is present in the optical transform plane. The dotted curve illustrates the result obtained with the stop present. 
4.1 Optical-digital system used for data acquisition. (a) System for recording and monitoring the video signal.

(b) Interfacing the video signal to the computer.

4.2 (a) Plot of the intensity profile in the transform plane of a far-field hologram of a random array of opaque discs. The separation between disc-pairs is characterized by a Gaussian probability density function (pdf); $\mu=460 \mu \mathrm{m}$ and $\sigma=30 \mu \mathrm{m}$. (b) Magnitude of the FFT of the intensity profile vs. the discrete transform variable $n$.

4.3 (a) Plot of |FFT $\left\{I_{F}\right\} \mid$ vs. $n$ after low pass filtering.

(b) The envelope function $I_{0}$ is obtained by computing the inverse FFT of the data in (a).

4.4 (a) Plot of $I_{F} / I_{0}$ vs. position. (b) Same as (a) except the random fluctuations at the left and right portions of the curve were removed by setting $I_{F} / I_{0}$ to unity.

152

4.5 (a) Magnitude of FFT $\left\{I_{F} / I_{0}\right\}$ vs. the discrete transform variable $n$. (b) Plot of the computed (-) density function and the theoretical (--) density function vs. displacement.

4.6 (a) Plot of the intensity profile in the transform plane of a far-field hologram of a random array of opaque discs. The separation between disc-pairs is characterized by a Gaussian pdf; $\mu=420 \mu \mathrm{m}$ and $\sigma=40 \mu \mathrm{m}$. (b) Plot of the intensity profile, $I_{F}$, divided by the envelope $I_{0}$.

4.7 (a) Same as $4.6 \mathrm{~b}$ except the random fluctuations at the left and right portions of the curve were removed. (b) Plot of the computed (-) density function and the theoretical (--) density function vs. displacement.

4.8 (a) Plot of the intensity profile in the transform plane of a far-field hologram of a random array of opaque discs. The separation between disc-pairs is characterized by a Gaussian pdf; $\mu=400 \mu \mathrm{m}$ and $\sigma=50 \mu \mathrm{m}$. (b) Plot of the intensity profile, $I_{F}$, divided by the envelope $I_{0}$. 
4.9 (a) Same as $4.8 \mathrm{~b}$ except the random fluctuations at the left and right portions of the curve were removed. (b) Plot of the computed (-) density function and the theoretical (--) density function vs. displacement.

4.10 (a) Plot of the intensity profile in the transform plane of a far-field hologram of a random array of opaque discs. The separation between disc-pairs is characterized by a Gaussian pdf; $\mu=460 \mu \mathrm{m}$ and $\sigma=60 \mu \mathrm{m}$. (b) Plot of the intensity profile, $I_{F}$, divided by the envelope $I_{0}$.

4.11 (a) Same as $4.10 \mathrm{~b}$ except the random fluctuations at the left and right portions of the curve were removed. (b) Plot of the computed (-) density function and the theoretical (--) density function vs. displacement.

4.12 (a) Plot of the intensity profile in the transform plane of a far-field hologram of a random array of opaque discs. The separation between disc-pairs is characterized by a Gaussian pdf; $\mu=490 \mu \mathrm{m}$ and $\sigma=70 \mu \mathrm{m}$. (b) Plot of the intensity profile, $I_{F}$, divided by the envelope $I_{0}$.

4.13 (a) Same as $4.12 \mathrm{~b}$ except the random fluctuations at the left and right portions of the curve were removed. (b) Plot of the computed (-) density function and the theoretical (--) density function vs. displacement.

4.14 (a) Same as $4.12 \mathrm{~b}$ except a relatively small portion of the random fluctuations at the left and right extremes of the curve were removed. (b) Plot of the computed ( - ) density function and the theoretical (--) density function vs. displacement.

4.15 (a) Plot of the intensity profile in the transform plane of a far-field hologram of a random array of opaque discs. The separation between disc-pairs is uniformly distributed between $385 \mu \mathrm{m}$ and $535 \mu \mathrm{m}$. (b) Plot of the intensity profile, $I_{F}$, divided by the envelope $I_{0}$. 
4.16 (a) Same as $4.15 \mathrm{~b}$ except the random fluctuations at the left and right portions of the curve were removed. (b) Plot of the computed (-) density function and the theoretical (-) density function vs. displacement.

4.17 (a) Intensity profile in the transform plane of a double-exposure far-field hologram of $79 \mu \mathrm{m}$ particles falling in water. (b) Plot of the intensity profile, $I_{F}$, divided by the envelope $I_{0}$.

4.18 (a) Same as $4.17 \mathrm{~b}$ except the random fluctuations at the left and right portions of the curve were removed. (b) Plot of the experimentally obtained density function which characterizes the velocity distribution.

4.19 (a) Plot of the intensity profile in the transform plane of a far-field hologram of a random array of opaque discs. The separation between disc pairs is Gaussian in two-dimensions; $\mu_{1}=\mu_{2}=450 \mu \mathrm{m}$ and $\sigma_{1}=\sigma_{2}=$ $35 \mu \mathrm{m}$. (b) Plot of the intensity profile, $I_{F}$, divided by the envelope, $I_{0}$.

4.20 (a) Same as $4.19 \mathrm{~b}$ except the random fluctuations at the left and right portions of the curve were removed. (b) Plot of the computed (-) marginal density function and the theoretical (-) density function vs. displacement.

5.1 (a) Plot of the computed ( $\longrightarrow$ ) density function and the theoretical (--) density function vs. displacement. This plot was obtained by using the procedure described in Chapter 4. (b) Plot of the approximate (-) density function obtained by computing the FFT of the of the intensity profile in the transform plane of a doubly exposed hologram. (The theoretical (--) density function is also included.)

5.2 (a) Real images obtained from a far-field hologram of glass microspheres. (b) Real images obtained from a far-field hologram of glass microballoons. Note that the images of the microballoons exhibit interference fringes.

B.1 Reconstructed images obtained from a four-exposure hologram of $80 \mu \mathrm{m}$ pollen particles. 
B.2. (a) Optical Fourier transform of a double-exposure hologram. (b) Optical Fourier transform of a fourexposure hologram.

C.1 (a) Reconstructed images obtained from a hologram of $50 \mu \mathrm{m}$ glass microspheres. The particles were translated horizontally by $d / 10$ during exposure. (b) Same as (a) except the particle translation was $d / 5$.

C.2 Same as Fig. C.1 except the particle translation was (a) $d / 2$ and (b) $d$. 


\section{Chapter I}

\section{Background and Introductory Remarks}

\subsection{Introduction}

Holography is well suited to the study of dynamic threedimensional particle fields and has been used extensively for this purpose. The ability to record an image in three dimensions, as provided by holography, offers a powerful method of particulate characterization. Particles throughout a sample volume can be recorded and the resulting hologram provides information on both size and positon for all particles in the sample at a given instant in time. In addition, particle velocities may be obtained if multiple exposures (or multiple frames) are recorded.

Both in-line and of $\mathrm{f}$-axis recording geometries have been used to produce particle field holograms. For the in-line geometry, the sample is coherently illuminated and thus a portion of the incident light is diffracted by the particles. The remaining portion of the incident illumination is undeviated from its original direction of propagation and forms the reference wave. The hologram is produced by recording the interference pattern which results from the interaction of the reference wave with the diffracted light. Typically, the recording plane is located in the far-field of any given particle in the sample, but within the near-field of the 
sample volume as a whole. Applying the far-field condition ensures a relatively large separation between the in-line real and virtual images. Thus, in the plane of the real image, the contribution from the virtual image appears as a uniform background, allowing the real image to be clearly observed. In the off-axis method of holography, a separate reference wave is added; hence, the real and virtual images are located on separate axes. Since the images are separate, there is clearly no need to record in the far field.

In the following sections both in-line far-field holography and off-axis holography are briefly reviewed. Each method has its advantages and disadvantages, therefore, the user must choose the appropriate geometry based on the requirements imposed by a particular application.

\subsubsection{In-line far-field holography}

In-line far-field holography has been and continues to be an important method in the study of particle fields. The first practical application involved the study of naturally occurring fog droplets having a range in size from $4 \mu$ to $200 \mu$ (Silverman et al. [1964], Thompson etal. [1966]). Since the initial application, a wide variety of particle fields have been analyzed using far-field holography including aerosols, marine plankton and gas bubbles in liquids. The success of the technique is indicated by the significant body of literature which has developed since the early 1960 's. The literature has been reviewed on several occasions 
(Thompson [1974], [1982], Trolinger [1975] and Thompson and Dunn [1980]) and the references contained in these articles provide a reasonably complete bibliography of the field up to 1982 . From 1982 up to the present, new results and applications have continued to appear in the 1iterature (see, e.g., Vikram and Billet [1983], [1984a], [1984b], Grabowski [1983], S1imani etal. [1984] and Stanton etal. [1984]). The procedures for recording a far-field hologram and subsequently forming the reconstructed image are relatively straightforward to implement. Hologram formation and image reconstruction are illustrated in Figs. 1.1a and b, respectively. For simplicity, plane wave illumination is assumed for both recording and image reconstruction although spherical wave illumination may be used to introduce magnification or demagnification into the system (Fourney etal. [1969], Bexon [1973], Cartwright [1980]). As mentioned in the introduction to this section, a far-field hologram is formed by coherently illuminating a particle sample and recording the intensity pattern that results from the interference of the light diffracted by the particles with the undeviated portion of the incident beam. The separation between a given particle and the recording medium, $z$, is chosen so that the far-field condition is satisfied, i.e.,

$$
z>\frac{(2 a)^{2}}{\lambda}
$$

where $2 a$ is the characteristic width of the particle and $\lambda$ is the 

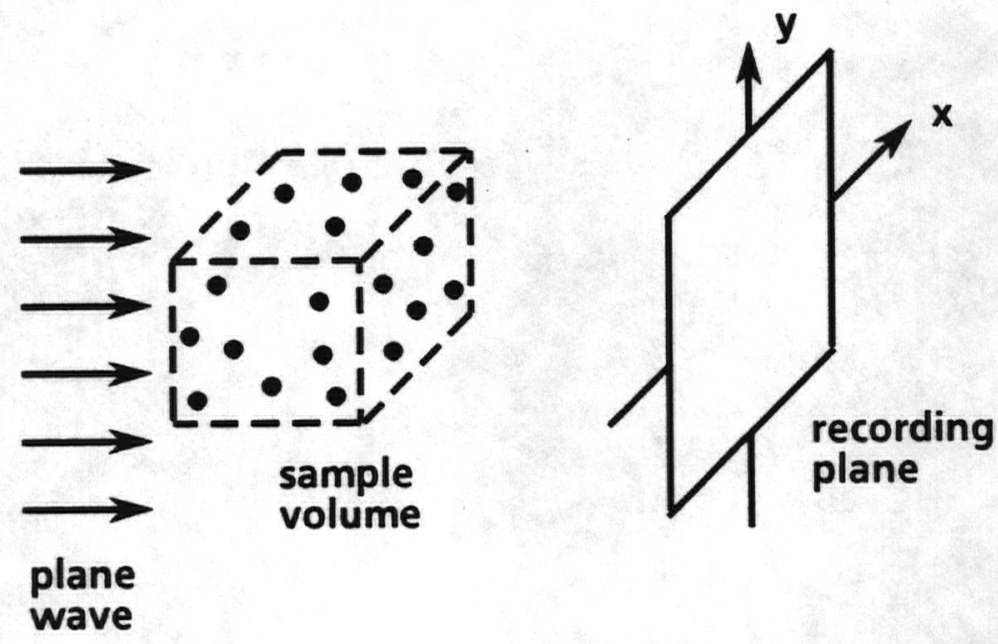

(a)

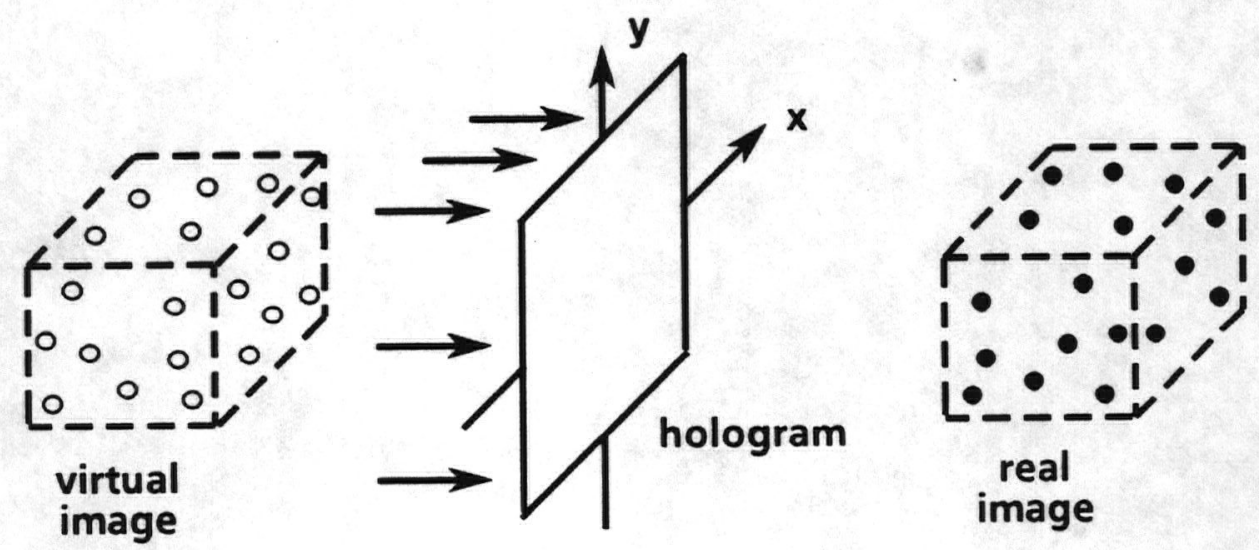

(b)

Fig. 1.1. (a) Geometry for recording a far-field hologram. (b) Hologram reconstruction. 
wavelength of the illuminating beam. It is often convenient to express the far-field condition in a slightly different manner namely,

$$
z=N \frac{(2 a)^{2}}{\Lambda}
$$

where $N$ is a constant greater than unity. The quantity $(2 a)^{2} / \lambda$ represents a distance of one "far field," thus, Equation (1.2) allows the object-to-hologram separation to be expressed as a multiple of this distance.

The individual particle images are formed by illuminating the hologram with a collimated beam of coherent 1 ight; real and virtual images of the particles comprising the sample are produced as shown in Fig. 1.1b. Clearly, either the real or virtual images may be viewed, however, the real image is primarily used in practice. Typically, a closed-circuit TV system is used to view the particle images, either by placing the vidicon directly in the plane of a given particle's image or by using a lens to relay the image (with magnification, if desired) to the vidicon.

The mathematical analysis describing the formation of a farfield hologram and the image reconstruction has been given by Tyler and Thompson [1976] and will not be repeated here. However, it is instructive to examine their expression for the transmittance function $t(x, y)$ of a hologram produced by a single particle: 


$$
\begin{aligned}
t(x, y)=B^{2}\left\{1-\frac{2}{\lambda z}\left[\sin \left(\frac{\pi \rho^{2}}{\lambda z}\right)\right.\right. & \left.\operatorname{Re} \tilde{A}\left(\frac{x}{\lambda z}, \frac{y}{\lambda z}\right)+\cos \left(\frac{\pi \rho^{2}}{\lambda z}\right) \operatorname{Im} \tilde{A}\left(\frac{x}{\lambda z}, \frac{y}{\lambda z}\right)\right] \\
& \left.+\frac{1}{\lambda^{2} z^{2}} \tilde{A}\left(\frac{x}{\lambda z}, \frac{y}{\lambda z}\right) \tilde{A}^{*}\left(\frac{x}{\lambda z}, \frac{y}{\lambda z}\right)\right\},
\end{aligned}
$$

where $x$ and $y$ are the coordinates in the hologram plane, $B$ is the amplitude of the plane wave used to illuminate the particle, $\lambda$ is the wavelength of the illumination, $z$ denotes the distance between the object and the recording plane and $\rho^{2}=x^{2}+y^{2}$. In addition, $\bar{A}(\cdot)$ is the Fourier transform of the object function $A(\xi, \eta)$, i.e.,

$$
\tilde{A}\left(\frac{x}{\lambda z}, \frac{y}{\lambda z}\right)=\iint_{-\infty}^{+\infty} A(\xi, \eta) \exp \left[-i \frac{2 \pi}{\lambda z}(x \xi+y \eta)\right] d \xi d \eta
$$

For example, the object function for an opaque spherical particle of radius $a$ has a value of unity within the circular cross-section of the particle and is zero otherwise. The corresponding Fourier transform of this object function is (see, e.g., Goodman [1968])

$$
\tilde{A}\left(\frac{x}{\lambda z}, \frac{y}{\lambda z}\right)=\pi a^{2}\left[\frac{2 J_{1}\left(\frac{2 \pi a \rho}{\lambda z}\right)}{\left(\frac{2 \pi a \rho}{\lambda z}\right)}\right]
$$

where $J_{1}$ denotes a Bessel function of the first kind and order one. Therefore, the transmittance of a hologram of an opaque spherical particle may be obtained by substituting Equation (1.5) into (1.3) yielding 


$$
\begin{aligned}
t(x, y)=1-\frac{2 \pi a^{2}}{\lambda z} \sin \left(\frac{\pi \rho^{2}}{\lambda z}\right)\left[\frac{2 J_{1}\left(\frac{2 \pi a \rho}{\lambda z}\right)}{\left(\frac{2 \pi a \rho}{\lambda z}\right)} \mid\right. \\
+\frac{\pi^{2} a^{4}}{\lambda^{2} z^{2}}\left|\frac{2 J_{1}\left(\frac{2 \pi a \rho}{\lambda z}\right)}{\left(\frac{2 \pi a \rho}{\lambda z}\right)}\right|^{2},
\end{aligned}
$$

For simplicity, the unimportant constant $B$ was set to unity in going from Equation (1.3) to (1.6).

The imaging properties of the far-field hologram may be readily understood by examining the above expression. It should be noted that the transmittance function of a zone lens is given by $1+b \sin \left(c p^{2}\right)$ where the coefficients $b$ and $c$ are constants for an ordinary zone lens (and $-1<b<1$ since the transmittance is assumed to be non-negative). The first two terms of Equation (1.6) are of the form of the zone lens transmittance function, however, the coefficient $b$ is not constant (it is proportional to $2 J_{1}(\cdot) /(\cdot)$ ). Thus a far-field hologram is essentially a modulated zone lens; the envelope function which modulates the sinusoidal zone lens is the Fourier transform of the object function. Illuminating an unmodulated zone lens with a plane wave will, of course, cause the incident illumination to diffract into two components: one component is a spherical wave converging to a real focus and the second is a spherical wave diverging from a virtual focus. The 
focal points are symmetrically located about the plane of the zone lens and lie on an axis perpendicular to that plane. As mentioned, the zone lens in a far-field hologram is modulated by the transform of the object function. Consequently, the hologram will diffract an incident plane wave in much the same way as a zone lens; however, the hologram will produce real and virtual images of the original object instead of the real and virtual point images produced by an unmodulated zone lens.

Another important aspect of in-line far-field holography involves the resolution requirements of the medium used to record the hologram. Since the argument of the zone lens term is proportional to $\rho^{2}$, the fringe frequency increases linearly with increasing values of $\rho$. Hence, neglecting other limiting factors, the frequency response of the recording medium will effectively limit the spatial extent of the zone lens. That is, the aperture of the zone lens will be finite. As with a conventional lens, the resolution in the image plane is determined (for a given focal length) by the size of the lens aperture. Thus, the resolution required of the recording medium may be obtained by determining the radial extent of the hologram which must be recorded in order to achieve an acceptable image. In most cases, an acceptable image is obtained if three side lobes of the envelope function, $\bar{A}(\cdot)$, are recorded. Therefore, the highest spatial frequency which must be recorded is equal to the frequency of the zone lens evaluated at the radial coordinate corresponding to the location of the third side 
1obe. For example, assuming a spherical particle of radius $a$, Cartwright etal. [1980] have shown that a film resolution of at least $2 / a$ lines per $\mathrm{mm}$ (where $a$ is measured in millimeters) is required to record a far-field hologram using the three side-lobe criterion.

In addition to the resolution requirement, good fringe visibility is required to form a hologram which will yield an acceptable reconstructed image. The high frequency fringes to be recorded (i.e., those within the third side lobe of the envelope function) will generally have a much lower visibility than the low frequency fringes within the central maximum (Dunn and Thompson [1982]). Hence, even if the film has a sufficiently high cut-off frequency, the low contrast response may prevent the high frequency fringes from being recorded. It is also important to note that, for a given particle, the fringe visibility in the hologram plane decreases as the object-to-hologram distance increases (Dunn and Thompson [1982]). Therefore, it is usually desirable to keep the hologram plane as close to the object as possible without violating the far-field condition when recording a hologram.

\subsubsection{Off-axis holography}

The off-axis geometry of interest is obtained by introducing a separate off-axis reference wave to the in-1ine hologram forming geometry shown in Fig. 1.1a. The object wave in this case consists of the light diffracted by the particles in addition to the undeviated portion of the plane wave used to illuminate the sample 
volume. Assuming linear recording, illuminating the hologram with the reference wave will reproduce the original object wave.

The separate real and virtual images obtained by using offaxis holography can be of advantage; however, any advantage must be weighed against the increased resolution required to record an offaxis hologram as compared to an in-1ine far-field hologram. To illustrate, it will be assumed for the moment that an off-axis hologram is recorded with no particles present in the sample volume. Thus, the resulting hologram is just a sine-wave grating resulting from the interference of the plane reference wave with the plane object wave. It may readily be shown that the spatial frequency of the sinusoidal fringes is given by

$$
f_{0}=\frac{\sin \theta}{\lambda}
$$

where $\theta$ denotes the angle of propagation of the reference wave with respect to the object wave and $\lambda$ is the wavelength of both beams. Using typical values of $30^{\circ}$ for $\theta$ and $500 \mathrm{~nm}$ for $\lambda$ yields a spatial frequency of 1000 lines per $\mathrm{mm}$. Hence, recording the planar object wave holographically requires a film cut-off frequency of at least 1000 lines per mm. When a particle is present, the cut-off frequency necessary to record an off-axis hologram increases substantially. For example, let us assume that an off-axis hologram of a single particle of width $2 a$ is to be formed and that the recording plane is in the far-field of the object. Clearly, a zone 
lens term is present in the off-axis case, but only an off-axis portion of that zone lens is recorded. The amount by which the zone lens is displaced from the axis is represented in frequency-space by the quantity $f_{0}$ given in Equation (1.7). To provide a direct comparison with the in-line far-field system, the three side lobe condition will be applied. Hence, the highest spatial frequency to be recorded is given by the sum of $f_{0}$ and the highest frequency necessary to record a far-field hologram out to the third side lobe of the envelope function, i.e.,

$$
\frac{\sin \theta}{\lambda}+\frac{2}{a}
$$

Thus, to record an off-axis hologram of a given particle, the cutoff frequency of the film must be $(\sin \theta) / \lambda$ lines per $\mathrm{mm}$ greater than that required to record an in-line hologram of the same particle.

Nonetheless, in certain applications involving the analysis of particle fields, the use of off-axis holography may be preferable to using in-line far-field holography. One example occurs when the particles comprising a given sample are characterized by a wide size distribution. Such a situation is encountered in the study of cavitation bubble fields where the bubble size may vary from about $10 \mu$ up to $1 \mathrm{~mm}$ (Haussmann and Lauterborn [1980]). To form a farfield hologram of such a sample, the recording plane would be placed at a suitable location in the far field of the largest particle contained in the sample volume. This, of course, implies that the 
recording plane would be located well within the far field of the smallest particle. Hence, the visibility of the fringes corresponding to the smallest particle would be very low and therefore difficult to record. Thus, the far-field condition cannot be satisfied for the largest particles in the sample if the smallest ones are to be recorded at the same time. Off-axis holography would be of advantage in this particular application since it is not necessary to apply the far-field condition.

off-axis holography is also of interest when the total Fraunhofer diffraction pattern of the reconstructed particle field is to be studied. The diffraction pattern of an in-line hologram has distinct differences when compared to the diffraction pattern of an off-axis hologram and this topic is addressed in Chapter 2.

\subsection{Particle velocity measurement using holography}

The measurement of particle velocities is of interest in many technical fields; some applications may require the velocity spectrum only, while others may require the size and position of the individual particles as well. In any case, a double (or multiple) exposure hologram can be of value since it contains size, position and displacement information for all particles within a given sample volume. Clearly, the velocity information may be obtained if the time interval between exposures is known.

Literature describing the use of far-field holography for velocimetry applications began to appear in the 1960's. Trolinger 
etal. [1968], [1969] and Fourney etal. [1969] outline the basic technique for recording a doubly exposed far-field hologram, including the requirements of the laser source with regard to producing a doublepulsed output. Shofner et al. [1969] and Menzel and Shofner [1970] discuss several methods for extracting the velocity information from a double-exposure hologram. In addition to the straightforward approach of measuring the particle displacements from the reconstructed holographic images, these authors discuss a method of analyzing the holographic record directly to obtain the required data as well as a method using optical processing. To understand how the holographic record may be interpreted directly, a doubleexposure far-field hologram of a single moving particle is considered. The holographic record in this case consists of two individual particle holograms, that is, one hologram corresponding to each exposure. The displacement between exposures may be obtained by measuring the center-to-center distance between the individual particle holograms. Clearly, this measurement gives the component of displacement in a plane parallel to the hologram plane. To obtain the component of displacement in the perpendicular direction (i.e., the $z$-direction), the holographic zone lens must be considered. Since the fringe spacing of the zone lens term is $z-$ dependent (see Equation (1.3)), the $z$-coordinate of the particle during each exposure may be calculated if the zeros of the respective zone lenses are measured. The displacement, $\Delta z$, is then obtained by taking the difference of the $z$ coordinate measured from 
the first exposure with that measured from the second exposure. In practice, however, Menzel and Shofner [1970] have found that $\Delta z$ may be measured to within $\pm 1 \mathrm{~mm}$ which is clearly too large an error for most applications. Since the resolution in the plane parallel to the hologram is inherently much better, the authors suggest the simultaneous recording of two, double-exposure holograms which are positioned at some angle relative to each other to obtain all three spatial coordinates with high accuracy.

\subsubsection{Applications}

A number of applications using in-line far-field holography as well as off-axis holography to measure particle velocities have been reported. Boettner and Thompson [1973] and LeBaron and Boettner [1980] have used double-exposure far-field holography to study various types of moving particulate matter including talc, asbestos and other pollutants. Studies on the relationship between particle shape and settling rate and the manner in which the various particles move through the atmosphere were carried out to better understand the nature of these pollutants. Carder [1979] used an in-line geometry to study particles found in ocean water. Rather than using multiple exposures, Carder recorded multiple frames to investigate the sizes, shapes and settling rates of the various particles. The shape and velocity of methanol droplets emerging from a nozzle were investigated by Uyemura etal. [197.8] using doubleexposure far-field holography. An off-axis arrangement was used by 
Hinsch etal. [1974] to study the dynamics of cavitation bubble fields. The authors recorded two separate holograms on a single plate by using a separate reference beam for each exposure. Acousto-optic cells were used to quickly switch from one reference beam to the next so that the exposures could be made in rapid succession.

When a double-exposure hologram of a sample containing a large number density of particles is reconstructed, it may be difficult to pair up a given particle image with the corresponding image related by translation. To overcome this problem Murakami and Ishikawa [1978] developed a far-field holographic system using two lasers operating at different wavelengths. A combination of mirrors and beam splitters were used to make the beam paths colinear, allowing the sample volume to be illuminated in the same direction by both lasers. The lasers were fired consecutively with a preselected time interval between exposures. Instead of using the conventional inline far-field recording geometry, interference filters were used to separate the two wavelengths and two far-field holograms were recorded. After development, each holographic plate is illuminated with collimated laser light of a different wavelength and the resulting images are superimposed. As a result, each particle of a given particle pair is of a diffferent color, hence, it is relatively easy to select the particle pairs related by tranlsation. 


\subsubsection{Automated readout}

Analyzing the reconstructed images from a particle field hologram can become tedious and time consuming when the sample volume of interest contains a large number of particles, thus there is a need for automated readout. Automatic analysis of the hologram would be desirable not only to facilitate data acquisition, but to handle situations intractable by manual analysis, thereby extending the usefulness of the holographic method itself.

Automatic assessment of particle field holograms has been investigated by a number of authors. For example, Bexon et al. [1976] described a method in which a digital image analyzer is programmed to measaure the size and location of all particles in the reconstructed image volume of a particle field hologram. Ewan [1979a], [1979b] described an optical-digital processing method in which the optical Fourier transform of a doubly exposed far-field hologram is analyzed by digital means to obtain the velocity distribution. Also, Shofner etal. [1969] described a method employing optical matched filtering to determine the position of a given particle directly from a far-field hologram. To implement this technique, a filter which is matched to a far-field hologram produced by an individual particle is generated. This filter may then be used to detect the presence of all such particle holograms present in a composite far-field hologram produced by a sample containing many particles. It is important to note that even if a 
hologram of a sample containing indentical particles is used as input to the matched filtering system, the output of the system will not necessarily produce correlation peaks corresponding to each individual particle hologram. Since the transmittance function of a far-field hologram is $z$-dependent (see Equation (1.3)), the output of the optical processor will yield a correlation peak for only those holograms having the $z$-location to which the filter is matched. Thus, in this manner, optical processing may be used to measure the individual particle locations.

Among the methods of automated readout outlined above, the technique employing the matched filters is clearly the least practical since a prohibitively large set of filters would, in general, be required to obtain the positions of all particles within a given sample volume. In fact, it is likely that manual readout could be performed more efficiently in most cases than the optical processing method. In comparison, the digital and optical-digital methods have the potential to provide relatively fast and reliable means of data acquisition.

\subsubsection{Digital vs. optical-digital processing of data from particle field holograms}

In performing a digital analysis of the holographic reconstruction of a particle field, the computer is used to emulate the human observer in locating and classifying the individual particle images. The apparatus used to process the holographic data typically includes a TV camera, image digitizer, an $x-y-z$ translator 
on which the hologram is mounted and, of course, a computer. The reconstructed sample volume is scanned automatically by operating the translator under computer control while the image digitizer allows the computer to "see" that portion of the reconstruction which is imaged onto the vidicon. Individual particle images will go in and out of focus as the sample volume is scanned; hence, it is necessary to include software which allows the computer to decide when a particle image is brought into focus. Once the particle image is in focus, appropriate software can be used to obtain the parameters of interest including particle size and position (see, for example, Bexon etal. [1976]).

In addition to Bexon, other workers have applied digital methods to the analysis of particle field holograms. For example, Haussmann and Lauterborn [1980] used digital image processing to analyze holographic images of gas bubbles in liquid. Although neither of these authors used the technique to make velocity measurements, Payne et al. [1984] did succeed in applying digital analysis to obtain particle velocities from the reconstructed images of a sequence of far-field holograms. The procedure used by Payne et al. was semiautomatic in that the particle images were manually brought into focus. The size, shape and position of each particle image were obtained with the help of a computer. By analyzing a sequence of holograms in this manner, these authors were then able to compute the displacement of a given particle between successive frames and hence obtain its velocity. 
The optical-digital (or hybrid) system first reported by Ewan [1979a], [1976b] for measuring particle velocity distributions is fundamentally different from the digital approach described above. Whereas the displacements of the particles are individually computed when using the digital technique, the hybrid system computes the distribution of displacements from the optical Fourier transform of a doubly exposed far-field hologram without directly considering the individual particle displacements. To understand how the hybrid system works, it is perhaps easiest to consider a doubly exposed far-field hologram of a sample for which all particles have the same velocity (in both magnitude and direction) and the direction of motion is parallel to the hologram plane. Thus, the holographic record consists of two identical far-field holograms in which one hologram is shifted from the other. In the Fourier transform plane of the doubly exposed hologram, a set of consinusoidal fringes is produced as a result of two-beam interference (each beam being produced by one of the holograms). Clearly, the spacing of the fringes is directly related to the displacement of the particles between exposures. (In addition to these fringes, other structure is present in the transform plane and is discussed in detail in Chapters 2 and 3.$)$ If the particles exhibit a distribution of velocities, the resultant fringe pattern is described by a summation of cosinusoidal terms in which the frequency of a given cosinusoid corresponds to the displacement of a given particle. Therefore, an 
analysis of the intensity pattern in the transform plane can, in principle, yield the velocity distribution function.

The hybrid system has several advantages over the digital approach; one advantage is that data acquisition is inherently faster with the optical-digital method since the particle displacements are not measured individually. Also, when measuring the particle displacements (manually, or digitally) from the holographic reconstruction, it will many times be difficult to pair up the particle images related by translation. Of course, the twowavelength method employed by Murakami and Ishikawa [1978] (see Section 1.2.1) may be used to help distinguish the particle pairs. However, the operation of the hybrid readout system depends only on the correlation of the particle field from one exposure to the next (Ewan [1979a]); thus, the velocity distribution is obtainable without having to determine which particle pairs are related by translation.

Finally, it should be noted that the hybrid system has at least one potential disadvantage, namely, spatial information regarding the individual particles is effectively lost. Obtaining the optical transform expresses the velocity information in a readily interpretable form, however, the random particle locations produce a speckle pattern in the transform plane from which the spatial information is not readily recovered. 


\subsection{Other optical techniques for measuring particle velocities}

Laser-Doppler velocimetry and laser speckle photography are other important optical methods for obtaining velocity measurements and are briefly reviewed in the following sections. The capabilities of these techniques as compared to the holographic method are also addressed.

\subsubsection{Laser-Doppler velocimetry}

Laser-Doppler velocimetry (LDV) is a well-established method for measuring particle velocities and, with particle seeding, it may be used to measure a wide variety of flows. (Durst et al. [1976], Stevenson et al. [1977]). The manner in which the LDV measures particle velocities is quite different from that of the holographic methods previously described. To compare the two techniques, it is probably best to begin by describing the method of operation of the LDV.

The optical arrangement for a typical LDV consists of two mutually coherent beams which intersect as shown in Fig. 1.2; the region of overlap of the two beams is called the probe volume. of course, interference fringes are formed within the probe volume and a straightforward calculation gives an expression for the fringe spacing:

$$
s=\frac{\lambda}{2 \sin \theta / 2}
$$




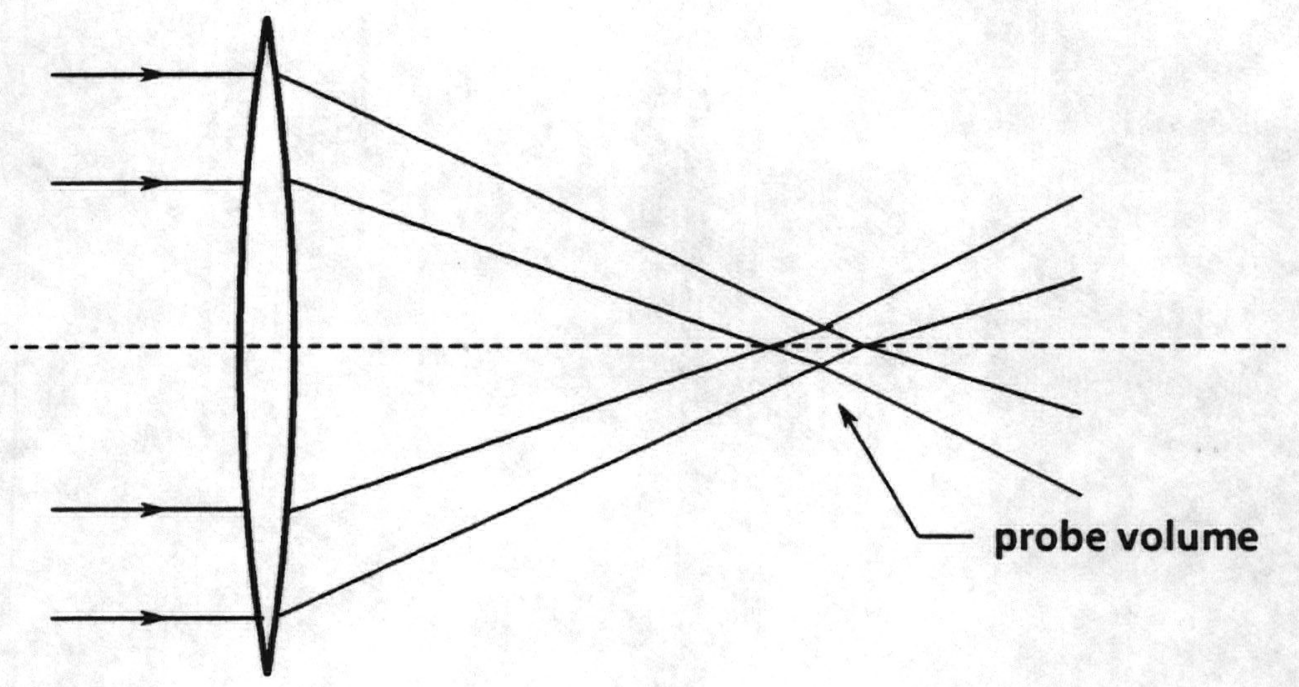

(a)

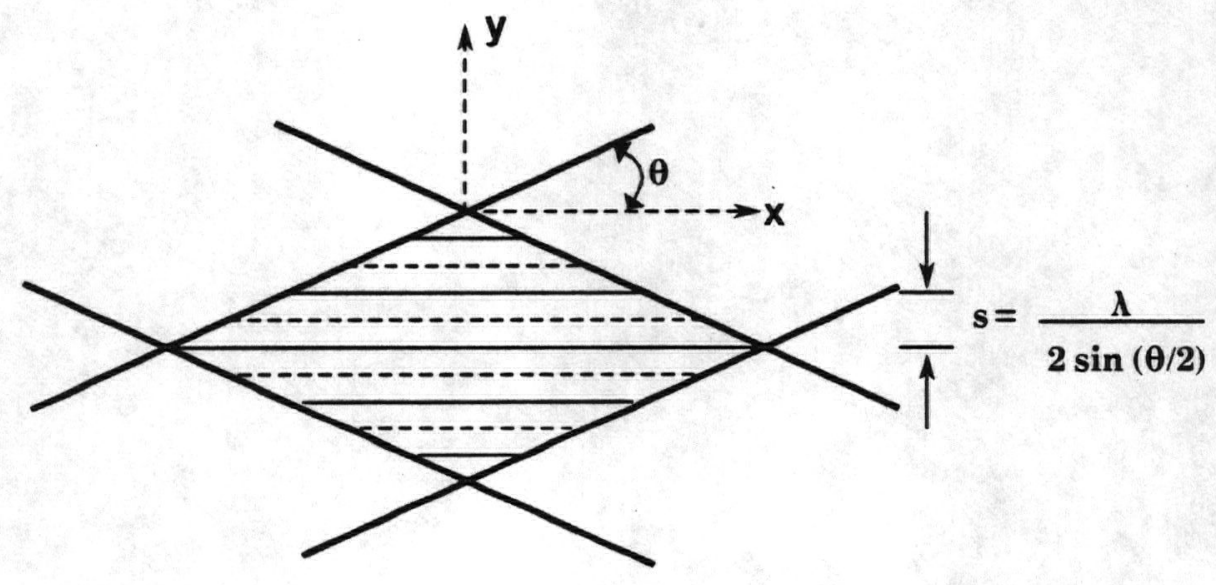

(b)

Fig. 1.2. (a) Optical arrangement for laser-Doppler velocimeter.

(b) Enlarged view of probe volume showing interference fringes. 
where $\lambda$ is the wavelength of the illumination and $\theta$ is the angle of intersection of the two beams. As a particle passes through the probe volume, it will scatter a maximum amount of 1 ight when it is in the region of a bright fringe, and correspondingly, it will scatter a minimum amount of light in the region of a dark fringe. Clearly, the intensity of the scattered light will fluctuate at a rate which is directly proportional to the $y$-component of the particle's velocity (the fringes are parallel to the $x$-axis in Fig. 1.2). If the frequency of the intensity fluctuations is denoted by $\Delta v$, the $y$-component of velocity is equal to the product of the fringe spacing and $\Delta v$, i.e.,

$$
v_{y}=s \Delta v=\frac{\lambda \Delta v}{2 \sin \theta / 2}
$$

(It should be noted that the above expression could also be derived by considering the Doppler shift of the scattered light from each beam as the particle passes through the probe volume (see, for example, Stevenson [1977]). Thus, velocity measurement is straightforward; once the system parameters $\theta$ and $\lambda$ are established, the appropriate electronics to measure $\Delta v$ are all that is required.

The light scattered by a given particle passing through the probe volume has properties which are particle-size dependent (as well as velocity dependent) and this has led to the interesting possibility of making simultaneous size and velocity measurements (Farmer [1972|, Bachalo and Houser [1984|). 
From the preceding discussion, it should be apparent that the laser-Doppler velocimeter measures particle velocities (and sizes) at a given locaton in a flow field as a function of time. In contrast, a double-exposure hologram contains size and velocity information for all particles within the recorded sample volume at a given instant in time. Therefore, these methods are not merely two ways of performing the same task; the choice of which method to use clearly depends on the type of measurement required.

\subsubsection{Laser speckle photography}

Laser speckle photography (Dudderar and Simpkins [1977], Simpkins and Dudderar [1978]) is similar to double-exposure holography in that instantaneous velocities throughout a region of a flow field are obtained. The speckle technique is implemented by first illuminating a planar region of a particle field with a laser whose output beam is expanded with the help of a cylindrical lens to form a "sheet of light." Since the particles are moving, they produce a time varying speckle pattern. A camera is focussed on the plane defined by the sheet of 1 ight and a double-exposure photograph of the resulting speckle pattern is then recorded. It is assumed that the time interval between exposures is short enough so that the speckle patterns remain correlated from one exposure to the next. The velocity information is obtained from the developed film by illuminating one portion at a time with laser light, forming the Fraunhofer diffraction pattern, and evaluating the spacing and 
orientation of the resulting parallel fringes. In this manner, a two-dimensional map of the velocity field may be obtained.

In comparing laser speckle photography to double-exposure holography, is has already been noted that each technique provides a means for performing velocity measurements throughout a flow field at a given instant in time. Since the double-exposure hologram and the speckle photograph can be analyzed in a similar manner (i.e., using transform methods) to obtain the velocity information, the data reduction time is probably comparable for both methods. However, if particle shape, size and location are also of interest, a speckle photograph does not contain this information whereas the hologram does. Clearly, the choice of which method to use depends on the application.

\subsection{Thesis content}

In this thesis particle velocimetry using double-exposure holography is investigated. In particlar, the optical-digital method proposed by Ewan is re-examined; a theoretical description which is more complete than the original analysis (Ewan [1979a]) is presented and new experimental results are given.

In chapter 2 , the theory of a single-exposure particle field hologram and the Fourier transform of the reconstructed images is presented. Both in-line and off-axis holograms are considered. It is shown that a zone lens appears in the transform plane of an inline hologram as a result of the on-axis images. Since the real and 
virtual images produced by an off-axis hologram do not lie on the same axis, a zone lens is not observed in the transform plane. However, it is shown that the off-axis hologram must be recorded linearly since nonlinear recording can produce in-line false images which yield a zone lens in the transform plane.

In Chapter 3, the theory developed in Chapter 2 is extended to describe the optical Fourier transform of a double-exposure hologram. A method for determining the distribution of particle displacements from intensity data measured in the transform plane is then presented and experimental results are given in Chapter 4

Finally, conclusions and suggestions for further research are given in Chapter 5. 


\section{Chapter II}

\section{Fourier Transforms of Reconstructed Images \\ from Particle Holograms}

\subsection{Introduction}

In this chapter, Fourier transforms of the reconstructed images from singly-exposed particle holograms are examined. While a single-exposure hologram does not contain particle velocity information, it is useful to discuss the single exposure hologram and the corresponding Fourier transform since the analysis can be readily extended to describe the double-exposure case (which, of course, does contain velocity information if the time interval between exposures is known).

Furthermore, the Fourier transform of the reconstructed images of a singly-exposed hologram is important in its own right since such a hologram may be used as input to a diffraction-based particle sizer. For example, the idea of using a hologram as a calibrated input to a sizing instrument has been suggested by Thompson [1984] . Various diffraction-based particle sizers have been described in the literature (see, for example, Dodge [1984], Cornillault [1972], Wertheimer et al. [1978] and Swithenbank et al. [1977]) and sizing instruments manufactured by Leeds and Northrup and Malvern Instruments are commercially available. Particle sizing in these 
instruments is accomplished by forming the composite (or total) diffraction pattern of a polydisperse field of particles. The Fraunhofer diffraction pattern is then analyzed electronically to yield the particle size distribution.

As noted by Ewan [1980] and Ewan et al. [1984], using a holographic record in place of the actual particle field allows for the measurement of size distributions of transient fields. Thus, the time resolution of the particle sizer is increased; it is effectively equal to the pulse length of the laser used to record the hologram. Ewan successfully applied this technique to the measurement of aerosol particles associated with a fuel injector nozzle, however, he observed that applying an in-line far-field hologram as input to the particle sizer is not entirely equivalent to using an actual particle sample as input. Ewan noted that the self-modulation term in the holographic record (i.e., $\bar{A}^{(\cdot)} \bar{A}^{*}(\cdot)$ in Equation (1.1)) provides an additional contribution to the composite diffraction pattern which, of course, is not present when an actual particle sample is used. In addition, a zone lens is present in the Fourier transform of an in-line hologram which does not appear in the total Fraunhofer diffraction pattern of a particle field. However, the analysis given by Ewan does not account for the zone lens. It is shown in section 2.2 that the zone lens is a direct result of the twin, on-axis images characteristic of in-line holography, which suggests that the zone lens will be absent if offaxis holography is used. This leads to section 2.3 in which the 
off-axis hologram and the corresponding Fourier transform are analyzed. It is shown that the zone lens is not present in the transform plane if the hologram is recorded linearly.

\subsection{The Fourier transform of a singly-exposed in-line hologram}

Two approaches may be considered when computing the Fourier transform of the reconstructed image of an in-line hologram. One approach is to treat the hologram as a transparency with a transmittance function $t(x, y)$ and then the transform of $t(x, y)$ can be evaluated. Hence, the imaging properties of the hologram are not explicitly considered in this situation. An in-line hologram will, of course, yield real and virtual images which lie on an axis perpendicular to the hologram plane. Therefore, the second approach is to evaluate the Fourier transform based on the knowledge of the twin images and their respective locations. Thus, for calculation purposes, the hologram is effectively replaced by the images it produces. The two methods are not entirely equivalent since, as mentioned in Section 2.1 , the holographic record contains a selfmodulation term in addition to the image forming terms. The former approach automatically accounts for the self-modulation term while the latter does not. Hence, the former will be used in the analysis to follow.

2.2.1 The transform of a hologram of a single particle

The theory is probably best understood if the Fourier transform of an in-line hologram of a single, opaque particle is 
analyzed first. The analysis will then be extended in the following section to describe the transform of a hologram of many particles. The configuration used for recording an in-line hologram is shown in Fig. 2.1a. The object consists of a single particle that is illuminated with a collimated beam of coherent light. The recording (or hologram) plane is located a distance $z$ away from the object. The complex field distribution, $\Psi_{H}(x, y)$, in the hologram plane is determined by the Huygens-Fresnel Principle and is given by

$$
\begin{aligned}
\Psi_{H}(x, y)= & -\frac{i}{\lambda z} \exp [i k z] \\
& \times \iint_{-\infty}^{+\infty}[1-A(\xi, \eta)] \exp \left\{i \frac{k}{2 z}\left[(x-\xi)^{2}+(y-\eta)^{2}\right]\right\} d \xi d \eta .
\end{aligned}
$$

Since the particle is assumed to be opaque, the function $A(\xi, \eta)$ describes the particle's cross-sectional shape. In addition, $\xi$ and $\eta$ denote the coordinates in the object plane; $x$ and $y$ denote the coordinates in the hologram plane. $k$ is the propagation constant which, for free space, is equal to $2 \pi / \lambda$ where $\lambda$ is the wavelength of light used to form the hologram. After a straightforward integration, equation (2.1) may be rewritten as

$$
\begin{aligned}
\Psi_{H}(x, y) & =\exp [i k z] \\
& +i \frac{\exp (i k z)}{\lambda z} \iint_{-\infty}^{+\infty} A(\xi, \eta) \exp \left\{i \frac{k}{2 z}\left[(x-\xi)^{2}+(y-\eta)^{2}\right]\right\} d \xi d \eta
\end{aligned}
$$




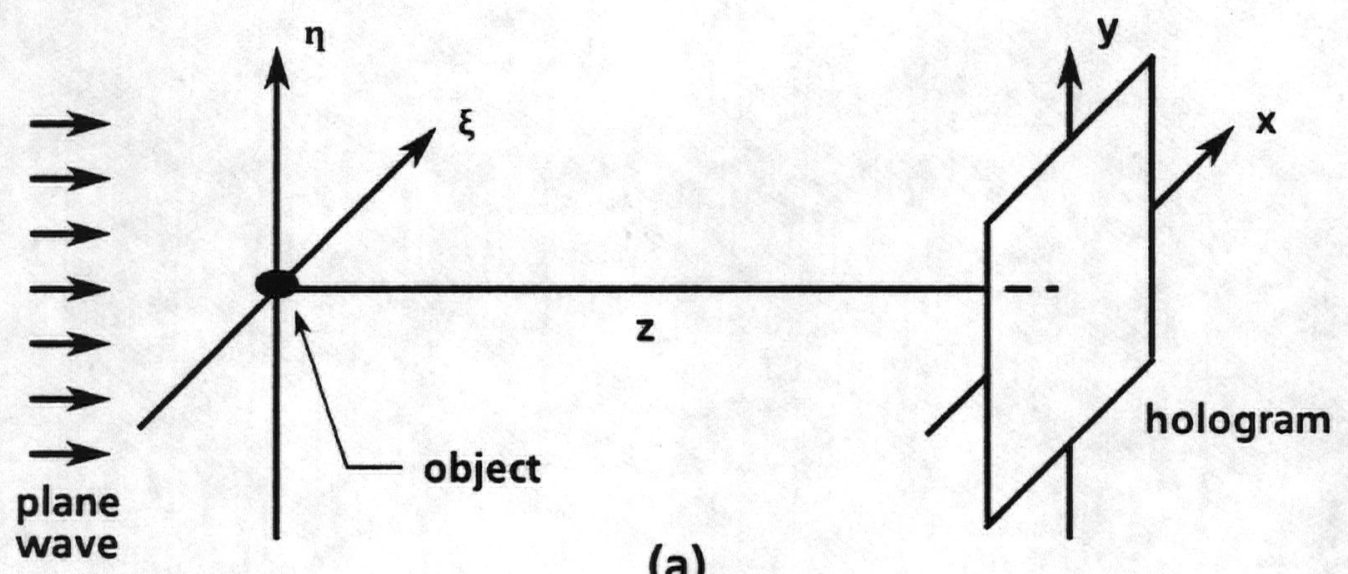

(a)

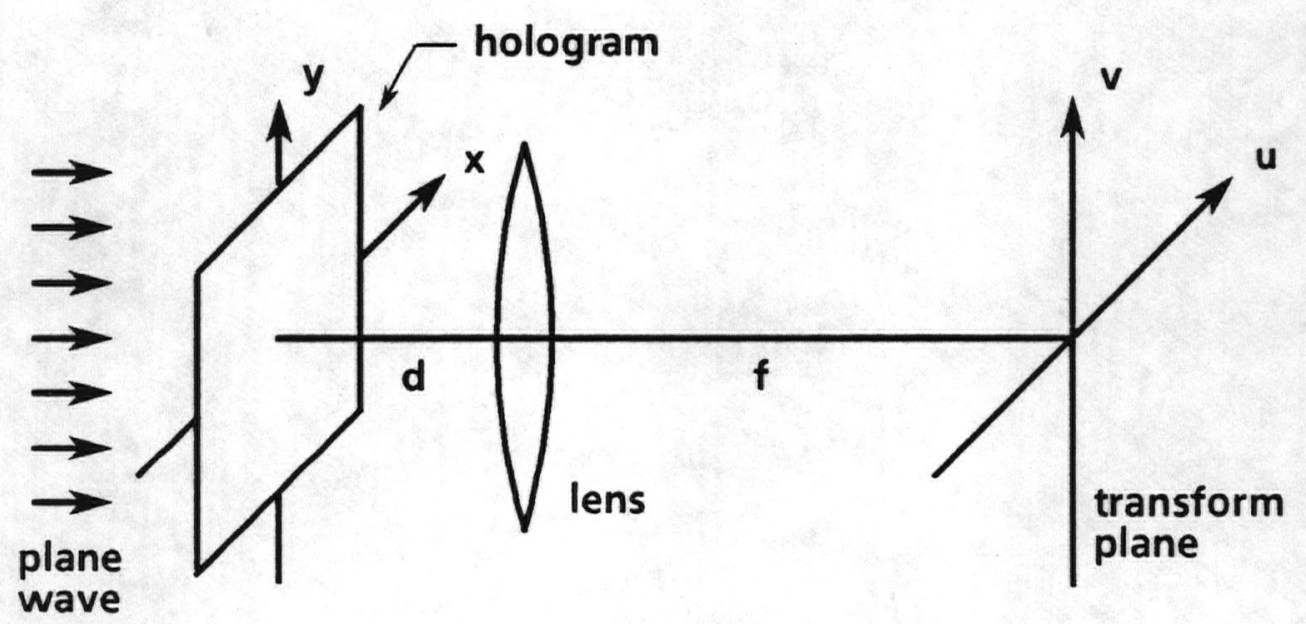

(b)

Fig. 2.1. (a) Hologram recording geometry. (b) Geometry for forming the optical Fourier transform. 
If the hologram is formed in the far field of the object, the integral in Equation (2.2) can be approximated by applying the farfield condition (Tyler and Thompson [1976]). However, Equation (2.2) is valid in the Fresnel (near) zone as well as the the Fraunhofer (far) zone and this more general form will be used in the Fourier transform calculations which follow. Indeed it will be shown that the transform of an in-line hologram has the same functional form regardless of whether it was recorded in the Fresnel zone or the Fraunhofer zone. However, it will be shown that it is usually necessary to record in the far zone in order to obtain useful data from the intensity pattern in the transfrom plane of the hologram. Furthermore, if the reconstructed holographic image and the Fourier transform are both needed for a particular application, it would clearly be advantageous to record the hologram in the far field to prevent confusion of the real image with the contribution from the virtual image.

To compute the Fourier transform of the hologram, the amplitude transmittance function of the hologram must first be calculated. The hologram is assumed to be recorded linearly, therefore, the amplitude transmittance, $t(x, y)$, will be equal to the intensity, $I_{H}(x, y)$, in the recording plane to within a multiplicative constant. The constant of proportionality is unimportant and is set to unity for convenience. Hence, the transmittance function is

$$
t(x, y)=I_{H}(x, y)=\Psi_{H}(x, y) \Psi_{H}^{*}(x, y)
$$


where the asterisk denotes a complex conjugate. Substituting Equation (2.1) into Equation (2.3) gives an expression for the transmittance in terms of the parameters used in recording the hologram, i.e.,

$$
\begin{aligned}
t(x, y) & =1+\frac{i}{\lambda z} \iint_{-\infty}^{+\infty} A(\xi, \eta) \exp \left\{i \frac{\pi}{\lambda z}\left[(x-\xi)^{2}+(y-\eta)^{2}\right]\right\} d \xi d \eta \\
& -\frac{i}{\lambda z} \iint_{-\infty}^{+\infty} A^{*}(\xi, \eta) \exp \left\{-i \frac{\pi}{\lambda z}\left[(x-\xi)^{2}+(y-\eta)^{2}\right]\right\} d \xi d \eta \\
& +\frac{1}{\lambda^{2} z^{2}} \iiint \int_{-\infty}^{+\infty} A(\xi, \eta) A^{*}\left(\xi^{\prime}, \eta^{\prime}\right) \\
& \times \exp \left\{i \frac{\pi}{\lambda z}\left[(x-\xi)^{2}+(y-\eta)^{2}-\left(x-\xi^{\prime}\right)^{2}-\left(y-\eta^{\prime}\right)^{2}\right]\right\} d \xi d \xi d \eta d \eta^{\prime}
\end{aligned}
$$

The Fourier transform of the hologram is to be performed optically as shown in Fig. 2.1b. The expression relating the field in the transform plane, $\Psi_{F}(u, v)$, to the holgram transmittance, $t(x, y)$, is (Goodman [1968])

$$
\Psi_{F}(u, v)=\frac{\exp \left[i \frac{k}{2 f}\left(1-\frac{d}{f}\right)\left(u^{2}+v^{2}\right)\right]}{i \lambda f} \iint_{-\infty}^{+\infty} t(x, y) \exp \left[-i \frac{2 \pi}{\lambda f}(u x+v y)\right] d y d x,(2.5)
$$

where $u$ and $v$ are coordinates in the transform plane, $d$ is the separation between the hologram and the transform lens and $f$ is the focal length of the transform lens. (In writing Equation (2.5), it is assumed that a plane wave of unit amplitude is used to illuminate 
the hologram.) The field in the transform plane is obtained by substituting Equation (2.4) into (2.5) yielding

$$
\Psi_{F}(u, v)=T_{1}+T_{2}+T_{3}+T_{4}
$$

That is, $\Psi_{F}(u, v)$ is a sum of four terms corresponding to the four terms of Equation (2.4). The computations are lengthy, therefore, each term will be treated individually to make the problem manageable. Furthermore, the quadratic phase term of Equation (2.5) will be dropped since the intensity in the transform plane is the quantity of interest.

The first term, $T_{1}$, is essentially the two-dimensional Fourier transform of unity which, of course, is a two-dimensional Dirac delta function. Explicitly,

$$
T_{1}=-i \lambda f \delta(u, v)
$$

The second term is given by the following expression:

$$
T_{2}=\frac{1}{\lambda^{2} f z} \iiint \int_{-\infty}^{+\infty} A(\xi, \eta) \exp \{\phi\} d \xi d \eta d x d y
$$

where

$$
\phi=i \frac{\pi}{\lambda z}\left[(x-\xi)^{2}+(y-\eta)^{2}\right]-i \frac{2 \pi}{\lambda f}(u x-v y) .
$$

The argument of the exponential, $\phi$, can be written as 


$$
\begin{aligned}
\phi=i \frac{\pi}{\lambda z}\left\{\left[x-\left(\xi+\frac{z u}{f}\right)\right]^{2}+\left[y-\left(\eta+\frac{z v}{f}\right)\right]^{2}\right. \\
\left.-\left(\frac{2 z u \xi}{f}+\frac{2 z v \eta}{f}\right)-\frac{z^{2}}{f^{2}}\left(u^{2}+v^{2}\right)\right\} .
\end{aligned}
$$

Furthermore, making the change of variables,

$$
\begin{aligned}
& x^{\prime}=x-\xi-\frac{z u}{f} \quad \text { and } \\
& y^{\prime}=y-\eta-\frac{z v}{f},
\end{aligned}
$$

and interchanging the order of integration in Equation (2.8) yields

$$
\begin{aligned}
T_{2}= & \frac{\exp \left[-i \frac{\pi z}{\lambda f^{2}}\left(u^{2}+v^{2}\right)\right]}{\lambda^{2} f z} \iiint \int_{-\infty}^{+\infty} A(\xi, \eta) \\
& \times \exp \left[-i \frac{2 \pi}{\lambda f}(u \xi+v \eta)+i \frac{\pi}{\lambda z}\left(x^{\prime 2}+y^{\prime 2}\right)\right] d x^{\prime} d y^{\prime} d \xi d \eta
\end{aligned}
$$

The integrals with respect to $x^{\prime}$ and $y^{\prime}$ in the above equation can be expressed in terms of Fresnel integrals (Abramowitz and Stegun [1972] pp. $300-301)$. Explicitly,

$$
\ell=\int_{-\infty}^{+\infty} \exp \left[-i \frac{\pi}{\lambda z} x^{\prime 2}\right] d x^{\prime}=2 \int_{0}^{\infty} \exp \left[-i \frac{\pi}{\lambda z} x^{\prime 2}\right] d x^{\prime}
$$




$$
=\sqrt{2 \lambda z} \int_{0}^{\infty} \cos \frac{\pi}{2} t^{2} d t-i \sqrt{2 \lambda z} \int_{0}^{\infty} \sin \frac{\pi}{2} t^{2} d t
$$

where $t=[2 / \lambda z]^{\frac{1}{2}} x^{\prime}$. Since the upper 1 imit on both Fresnel integrals is infinity, they can be evaluated in closed form yielding

$$
\ell=\left[\frac{\lambda z}{2}\right]^{\frac{1}{2}}(1-i)
$$

Following this procedure once again gives the same result for the integration with respect to $y^{\prime}$. Thus, Equation (2.11) reduces to

$$
T_{2}=\frac{i}{\lambda f} \exp \left[-i \frac{\pi z}{\lambda f^{2}}\left(u^{2}+v^{2}\right)\right] \tilde{A}\left(\frac{u}{\lambda f}, \frac{v}{\lambda f}\right)
$$

where $\bar{A}(\cdot)$ denotes a Fourier transform as defined in Equation $(1.4)$

The third term of Equation (2.6) can be evaluated in a similar manner to yield

$$
T_{3}=\frac{i}{\lambda f} \exp \left[i \frac{\pi z}{\lambda f^{2}}\left(u^{2}+v^{2}\right)\right] \iint_{-\infty}^{+\infty} A^{*}(\xi, \eta) \exp \left[-i \frac{2 \pi}{\lambda f}(u \xi+v \eta)\right] d \xi d \eta
$$

Since it is assumed that $A(\xi, \eta)$ is real, $A(\xi, \eta)=A^{*}(\xi, \eta)$ and therefore,

$$
T_{3}=\frac{i}{\lambda f} \exp \left[i \frac{\pi z}{\lambda f^{2}}\left(u^{2}+v^{2}\right)\right] \tilde{A}\left(\frac{u}{\lambda f}, \frac{v}{\lambda f}\right) .
$$

The fourth term of Equation $(2.6)$ is 


$$
T_{4}=\frac{1}{i \lambda^{3} f z^{2}} \iiint \iiint_{-\infty}^{+\infty} A(\xi, \eta) A\left(\xi, \eta^{\prime}\right) \exp [a] d \xi d \xi^{\prime} d \eta d \eta^{\prime} d x d y
$$

where $A(\xi, \eta)$ is again assumed to be real and

$$
a=i \frac{\pi}{\lambda z}\left[(x-\xi)^{2}+(y-\eta)^{2}-\left(x-\xi^{\prime}\right)^{2}-\left(y-\eta^{\prime}\right)^{2}\right]-i \frac{2 \pi}{\lambda f}(x u+y v)
$$

The argument of the exponential can be rewritten as

$$
a=i \frac{\pi}{\lambda z}\left[2 x\left(\xi^{\prime}-\xi-\frac{z u}{f}\right)+2 y\left(\eta^{\prime}-\eta-\frac{z v}{f}\right)+\xi^{2}+\eta^{2}-\xi^{2}-\eta^{\prime 2}\right]
$$

The integrations with respect to $x$ and $y$ are performed first using the delta function indentity,

$$
\delta(\beta)=\int_{-\infty}^{+\infty} \exp [i 2 \pi f \beta] d f
$$

Integrating with respect to $\xi^{\prime}$ and $\eta^{\prime}$ then yields

$$
\begin{aligned}
T_{4}=\frac{i}{\lambda f} \exp \left[-i \frac{\pi z}{\lambda f^{2}}\left(u^{2}+v^{2}\right)\right] \int & \int_{-\infty}^{+\infty} A(\xi, \eta) A\left(\xi+\frac{z u}{f}, \eta+\frac{z v}{f}\right) \\
& \times \exp \left[-i \frac{2 \pi}{\lambda f}(u \xi+v \eta)\right] d \xi d \eta .
\end{aligned}
$$

The quantities $T_{2}$ and $T_{3}$ can be combined, allowing the field in the transform plane to be written as 


$$
\Psi_{F}(u, v)=-i \lambda f \delta(u, v)+\frac{2 i}{\lambda f} \cos \left(\frac{\pi z \rho^{2}}{\lambda f^{2}}\right) \bar{A}\left(\frac{u}{\lambda f}, \frac{v}{\lambda f}\right)+T_{4},
$$

where $\rho=\left[u^{2}+v^{2}\right]^{\frac{1}{2}}$. The significance of each of the terms in the above expression will now be discussed.

The delta function represents the portion of the light which is undiffracted by the hologram. It is clearly an idealization which results from ignoring the finite aperture effects of the optical system. However, assuming the clear aperture of the optical transform system is sufficiently large, a delta-like contribution will be present in practice. That is, a narrow, intense distribution of light (which is the Fourier transform of the aperture of the optical system) will be observed at the origin of the transform plane.

The second term in Equation (2.21) is of primary interest since it is essentially the Fraunhofer diffraction pattern of the original object. More precisely, it is a cosinusoidal zone lens term modulated by the Fourier transform of the original object function, $A(\xi, \eta)$. Needless to say, if the original object had been used in place of the hologram in the transform geometry of Fig. $2.1 \mathrm{~b}$, the zone lens would not be present. The presence of the zone lens may be explained with the help of Fig. 2.2. As a simplification, the object recorded on the hologram is assumed to be a point scatterer. The points $P_{0}$ and $P_{1}$ correspond to the virtual and real images, respectively, resulting from an in-line hologram of 


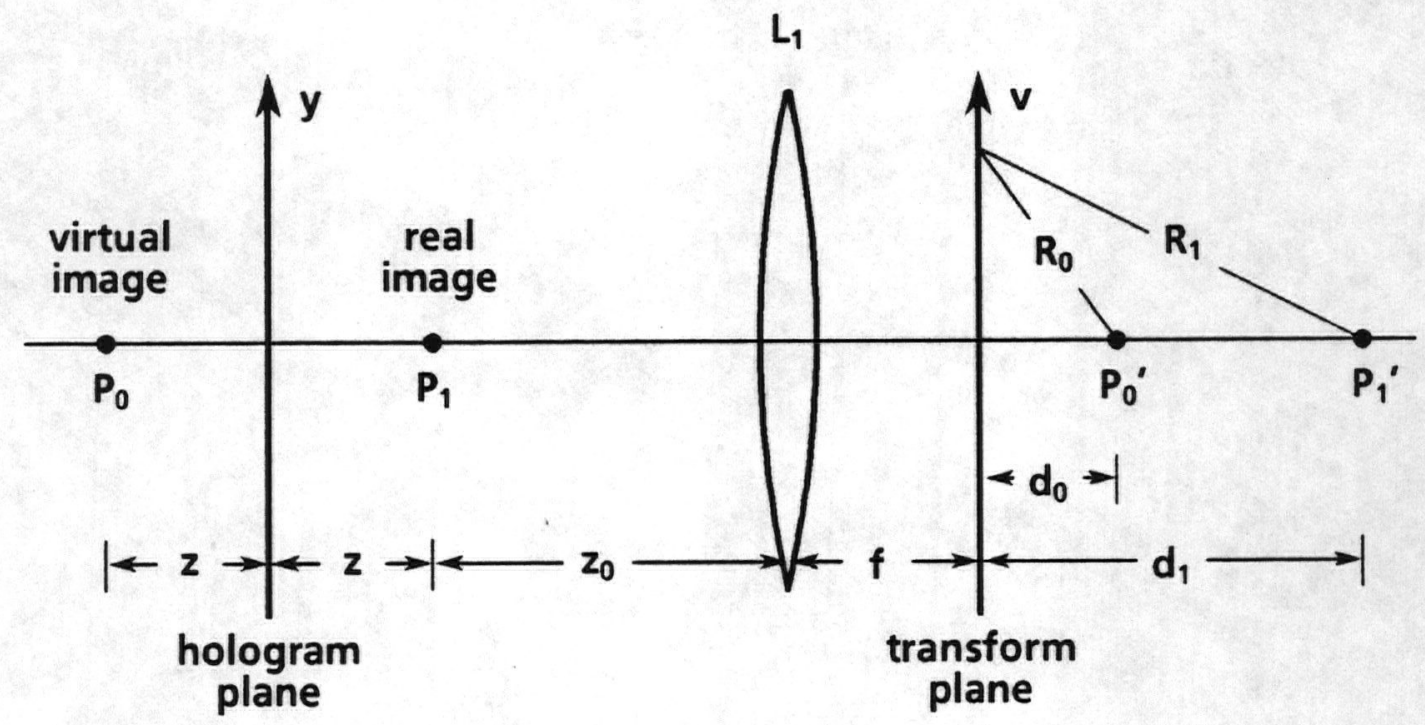

Fig. 2.2. The zone lens in the transform plane of an in-line hologram results from the interference of the spherical waves produced by the real and virtual images. 
a single point object. The separation between images is $2 z$ where $z$ is the original object-to-hologram separation. Lens $L_{1}$, which corresponds to the transform lens of Fig. 2.1b, will re-image $P_{0}$ to $P_{0}^{\prime}$ and $P_{1}$ to $P_{1}^{\prime}$ in accordance with the lens law of geometrical optics. Assuming perfect imaging, the 1 ight waves converging to the image points $P_{0}^{\prime}$ and $P_{1}^{\prime}$ will be spherical and their respective radii of curvature are denoted by $R_{0}$ and $R_{1}$ in Fig. 2.2. The interference between the two spherical waves produces an intensity pattern in the form of a zone lens. In the focal plane of lens $L_{1}$, the intensity may be written as

$$
I(\rho)=I_{0}\left[1+\cos \frac{2 \pi}{\lambda}\left(R_{1}-R_{0}\right)\right],
$$

where $I_{0}$ is a constant and $\rho$ is the radial coordinate $\left(\rho^{2}=u^{2}+v^{2}\right)$. An important characteristic of a zone lens is that its spatial frequency increases with increasing distance from its center. The frequency, $v(\rho)$, of the zone lens at any distance $\rho$ from the origin of the transform plane may be found by differentiating the argument of the cosine function and dividing by $2 \pi$ (Collier, Burckhardt and $\operatorname{Lin}[1971]$, p. 67), i.e.,

$$
v(\rho)=\frac{1}{2 \pi} \frac{\partial}{\partial \rho}\left[\frac{2 \pi}{\lambda}\left(R_{1}-R_{0}\right)\right]
$$

Expressions for $R_{0}$ and $R_{1}$ in terms of $\rho$ are readily obtained by inspection of Fig. 2.2, namely, 


$$
\begin{aligned}
& -41- \\
& R_{0}=d_{0}\left[1+\left(\frac{\rho}{d_{0}}\right)^{2}\right]^{\frac{1}{2}} \approx d_{0}+\frac{\rho^{2}}{2 d_{0}}, \\
& R_{1}=d_{1}\left[1+\left(\frac{\rho}{d_{1}}\right)^{2}\right]^{\frac{1}{2}} \approx d_{1}+\frac{\rho^{2}}{2 d_{1}} .
\end{aligned}
$$

Substituting Equations (2.24) into (2.23) yields

$$
v(\rho)=\frac{1}{\lambda} \frac{\left(d_{1}-d_{0}\right) \rho}{d_{1} d_{0}}
$$

The distances $d_{0}$ and $d_{1}$ may be found by applying the lens law, i.e.,

$$
\begin{gathered}
\frac{1}{2 z+z_{0}}+\frac{1}{d_{0}+f}=\frac{1}{f}, \\
\frac{1}{z_{0}}+\frac{1}{d_{1}+f}=\frac{1}{f} .
\end{gathered}
$$

Thus, solving the above equations for $d_{0}$ and $d_{1}$ and substituting into Equation (2.25) yields the frequency of the zone lens,

$$
v(\rho)=\frac{2 z \rho}{\lambda f^{2}}
$$

This result is equal to the frequency of the zone lens term of Equation (2.21). Initially, there may appear to be a discrepancy since the frequency obtained from Equation (2.21) by differentiating the argument and dividing by $2 \pi$ is equal to $\rho z / \lambda f^{2}$. However, it must 
be remembered that Equation (2.21) describes the amplitude in the transform plane, $\Psi_{F}(u, v)$, whereas the frequency of the zone lens is properly obtained from the intensity expression $\Psi_{F} \Psi_{F}{ }^{*}$. Since the results do agree in both cases, it is concluded that the zone lens results from interference between the contributions from the real and virtual images.

Finally, the remaining term, $T_{4}$, of Equation $(2.21)$ is examined. As a matter of formal interest, it is observed that this quantity resembles the ambiguity function which is encountered in radar signal processing (Woodward [1953]). $T_{4}$ results from Fourier transforming the self-modulation term of an in-line hologram and its contribution to the resulting diffraction pattern is usually undesirable. However, as shown in the following analysis, its effect can be minimized by properly choosing the recording distance when forming the hologram.

The behavior of the integral,

$$
\iint_{-\infty}^{+\infty} A(\xi, \eta) A\left(\xi+\frac{z u}{f}, \eta+\frac{z v}{f}\right) \exp \left[-i \frac{2 \pi}{\lambda f}(u \xi+v \eta) \mid d \xi d \eta\right.
$$

must be examined since $T_{4}$ is proportional to this quantity (see Equations (2.20) and (2.21)). The limits of integration formally extend to infinity, however, the actual area of integration is determined by the area of overlap of the object function, $A(\xi, \eta)$, with a shifted version of itself, $A(\xi+z u / f, \eta+z v / f)$. In this respect, the above expression is similar to the autocorrelation integral. 
Clearly, the shifted version of the object function is displaced from the unshifted one by $-z u / f$ units along the $\xi$-axis and by $-z v / f$ units along the $\eta$-axis. Once the area of overlap becomes zero, the above integral and, hence, $T_{4}$ will vanish. Since $u$ and $v$ are the coordinates in the transform plane, it follows that $T_{4}$ will not contribute to the intensity in the transform plane once $u$ and $v$ become large enough to cause the area of overlap to become zero. To determine when, in practice, $T_{4}$ might be expected to vanish, the specific example of a spherical particle of diameter $2 a$ is considered. The object in this case is described by the circ function, i.e., $A(\xi, \eta)=\operatorname{circ}\left\{\left[\xi^{2}+\eta^{2}\right]^{1 / 2} / a\right\}$ where

$$
\operatorname{circ}\left\{\left[\xi^{2}+\eta^{2}\right]^{1 / 2} / a\right\}= \begin{cases}1 & \text { for }\left[\xi^{2}+\eta^{2}\right]^{1 / 2}<a \\ 0 & \text { otherwise }\end{cases}
$$

The object function will just fail to overlap with its shifted version when

$$
\rho_{0}=\frac{2 a f}{z}
$$

where $\rho$ is the radial coordinate in the optical transform plane, $f$ is the focal length of the transform lens and $z$ is the object-tohologram separation. Substituting $z=N(2 a)^{2} / \lambda$ into Equation (2.28) yields 


$$
\rho_{0}=\frac{\lambda f}{2 N a}
$$

where $N$ denotes the number of the far-field distances. It should be recalled that the Fraunhofer diffraction pattern of a spherical object is described by the Airy function and the radial distance to the first dark ring is

$$
\rho_{A}=1.22 \frac{\lambda f}{2 a}
$$

Hence, the width of the second term in Equation (2.21) is $1.22 \mathrm{~N}$ times that of the last term, $T_{4}$. The contribution from $T_{4}$ will therefore be localized about the origin of the transform plane if $N$ is sufficiently large. That is, the object-to-hologram separation should be at least 10 far-field distances to minimize the contribution from $T_{4}$ in the transform plane of the hologram. It will be assumed that this condition is always satisfied.

\subsubsection{The transform of a hologram of many particles - case 1: Particles confined to a} plane parallel to the hologram plane.

The analysis of the preceding section will now be extended to describe the Fourier transform of an in-line hologram of many particles. The present discussion is limited to the case in which all particles are located in a plane parallel to the recording plane. It should be noted that the "hologram" is actually a recording of many individual particle holograms. That is, the 
diffracted light from each particle will interfere with the undiffracted background illumination to produce an interference pattern which is recorded on the holographic plate. In addition, the diffracted light from any given particle will interfere with diffracted light from all other particles in the sample, producing intermodulation terms in the resulting holographic record. However, the contributions from the intermodulation terms are small and will be ignored (see Appendix A). Thus, the complex field amplitude in the transform plane of the composite hologram is just a summation of the contributions from each of the individual particle holograms. The expression for the field in the transform plane of a hologram of a single particle is given in Equation 2.21; therefore, by inspection, the field produced by a hologram of $N$ particles may be written as

$$
\Psi_{\mathrm{F}}(u, v)=\sum_{n=1}^{N} i \frac{2}{\lambda f} \cos \left(\frac{\pi z \rho^{2}}{\lambda f^{2}}\right) \tilde{A}_{n}\left(\frac{u}{\lambda f}, \frac{v}{\lambda f}\right)
$$

where $\tilde{A}_{n}(\cdot)$ denotes the Fourier transform of the $n^{\text {th }}$ particle. It should be noted that the first and last terms of Equation (2.21) have been dropped in going to (2.31) and hence, the above expression is valid only at points away from the origin of the transform plane. If all particles in a given sample are identical, then $\bar{A}_{n}(\cdot)$ will differ from $\tilde{A}_{n^{\prime}}(\cdot)$ (where $n \neq n^{\prime}$ ) by a linear phase factor. This relationship results from applying the shift theorem of Fourier analysis, namely, if $\left(\xi_{n}, \eta_{n}\right)$ denotes the location of the $n$th particle 
with respect to the origin of the object plane, then

$$
\begin{aligned}
\tilde{A}_{n}\left(\frac{u}{\lambda f}, \frac{v}{\lambda f}\right) & =F\left\{A\left(\xi-\xi_{n}, \eta-\eta_{n}\right)\right\} \\
& =\exp \left[-i 2 \pi\left(u \xi_{n}+v \eta_{n}\right)\right] F\{A(\xi, \eta)\},
\end{aligned}
$$

where $F\{\cdot\}$ denotes a Fourier transform. Combining Equations (2.31) and (2.32) yields

$$
\Psi_{F}(u, v)=\tilde{A}\left(\frac{u}{\lambda f}, \frac{v}{\lambda f}\right) \sum_{n=1}^{N} \cos \left(\frac{\pi z \rho^{2}}{\lambda f^{2}}\right) \exp \left[-i 2 \pi\left(u \xi_{n}+v \eta_{n}\right)\right],
$$

where the unnecessary multiplicative constant $2 i / \lambda f$ is omitted and $F\{A(\xi, \eta)\}$ is replaced by the symbol $\vec{A}(\cdot)$. The intensity, $I_{F}(u, v)$, in the transform plane is

$$
I_{F}(u, v)=\Psi_{F} \Psi_{F}^{*}=\left|\tilde{A}\left(\frac{u}{\lambda f}, \frac{v}{\lambda f}\right)\right|^{2} \cos ^{2}\left(\frac{\pi z \rho^{2}}{\lambda f^{2}}\right) \sum_{n=1}^{N} \sum_{m=1}^{N} \exp \left[i\left(\Phi_{n}-\phi_{m}\right)\right],
$$

where $\phi_{n}=2 \pi\left(u \xi_{n}+v \eta_{n}\right)$. In general, the particles are randomly located throughout the object plane, i.e., the $\phi_{n}{ }^{\prime} s$ and $\phi_{m}{ }^{\prime} s$ are random variables. As a result, the double summation term in Equation (2.34) is random, but $|\tilde{A}(\cdot)|^{2}$ and $\cos ^{2}(\cdot)$ clearly are not. Thus the intensity in the transform plane is composed of a deterministic envelope modulating a random component (the double summation term). Naturally, the same type of result is encountered when analyzing the diffraction pattern which results from using an 
actual particle sample in place of the hologram. The only difference is that the zone lens appears in the transform plane of the hologram but not in the the transform plane of the actual particle sample.

Since the intensity $I_{F}(u, v)$ is random, its statistics are of interest, particularly the expected value and variance. At this point, it should be mentioned that the statistical characteristics of composite far-field diffraction patterns produced by particle fields are documented in the 1iterature (see, for example, Anderson [1982]). Also, Goodman [1984] gives an analysis of speckle patterns produced by a surface comprised of numerous isolated scattering areas, which is closely related to the problem at hand.

The expected value of the intensity in the transform plane of an in-line hologram may readily be obtained based on the results of Goodman and Anderson. For convenience, the following quantities are defined:

$$
\begin{gathered}
S=\sum_{n=1}^{N} \exp \left[-i \phi_{n}\right]=S_{1}-i S_{2} \\
S_{1}=\sum_{n=1}^{N} \cos \left[\phi_{n}\right] \\
S_{2}=\sum_{n=1}^{N} \sin \left[\phi_{n}\right]
\end{gathered}
$$

The $\phi_{n}$ 's are assumed to be uniformly distributed in the interval 
ranging from 0 to $2 \pi$ and statistically independent of one another. Usually, it is not overly restrictive to assume a uniform distribution of phase since large phase variations, greatly exceeding $2 \pi$, will occur in general. Thus our assumption only requires that the phase variations yield a distribution which is uniform on the fundamental interval ranging from 0 to $2 \pi$. The particle positions are not required to be uniformly distributed even though each $\phi_{n}$ is a function of the location of the $n^{\text {th }}$ particle. With the above assumptions in mind, the following expected values are easily calculated:

$$
\begin{aligned}
<S_{1}>= & <S_{2}>=<S_{1} S_{2}>=<S>=0, \\
& <S_{1}^{2}>=<S_{2}^{2}>=\frac{N}{2} .
\end{aligned}
$$

Therefore, $\left\langle S S^{*}\right\rangle=N$ and the expected value of Equation (2.34) immediately follows, i.e.,

$$
<I_{F}(u, v)>=N\left|\tilde{A}\left(\frac{u}{\lambda f}, \frac{v}{\lambda f}\right)\right|^{2} \cos ^{2}\left(\frac{\pi z \rho^{2}}{\lambda f^{2}}\right),
$$

To illustrate this result, two holograms of $50 \mu$ glass spheres were produced. Prior to recording the holograms, the particles were placed on a glass slide, thereby confining them to a plane. The first hologram was formed using an object-to-recording plane separation of $6 \mathrm{~cm}$; for the second hologram, the separation was $12 \mathrm{~cm}$. These figures correspond to 12 and 24 far-field distances, 
respectively, for $\lambda=5145 \AA$. Photographs of the optical Fourier transform patterns resulting from these holograms are shown in Figs. 2.3 and 2.4. For comparison, the far-field diffraction pattern produced directly by the same particle sample used in forming the holograms is given in Fig. 2.5. A zone lens is readily observed in the transform plane of each of the holograms (Figs. 2.3 and 2.4) but is, of course, absent in Fig. 2.5. The fringe frequency of the zone lens in Fig. 2.3 is lower than that of Fig. 2.4 since the object-torecording plane separation was smaller for the hologram used in obtaining the former figure. The overall envelope, $|\dot{A}(\cdot)|^{2}$, is an Airy pattern since the particles are spherical in shape.

\subsubsection{Some aspects of speckle in the transform plane of a particle-field hologram.}

As expected, speckle is observed in Figs. 2.3-2.4 (in addition to the Airy pattern and the zone lens) as a result of the random particle locations. For a large number of particles, the central limit theorem may be applied to obtain the statistical characteristics of the speckle pattern; as the number of particles approaches infinity, the random variables $S_{1}$ and $S_{2}$ (see Equations (2.35)) become asymptotically Gaussian (Anderson [1982], p. 99). The usual techniques for transforming random variables may then be applied to show that the intensity in the transform plane, which is proportional to $S S^{*}$, is described by a negative exponential probability distribution function (Goodman [1984], p. 16). 


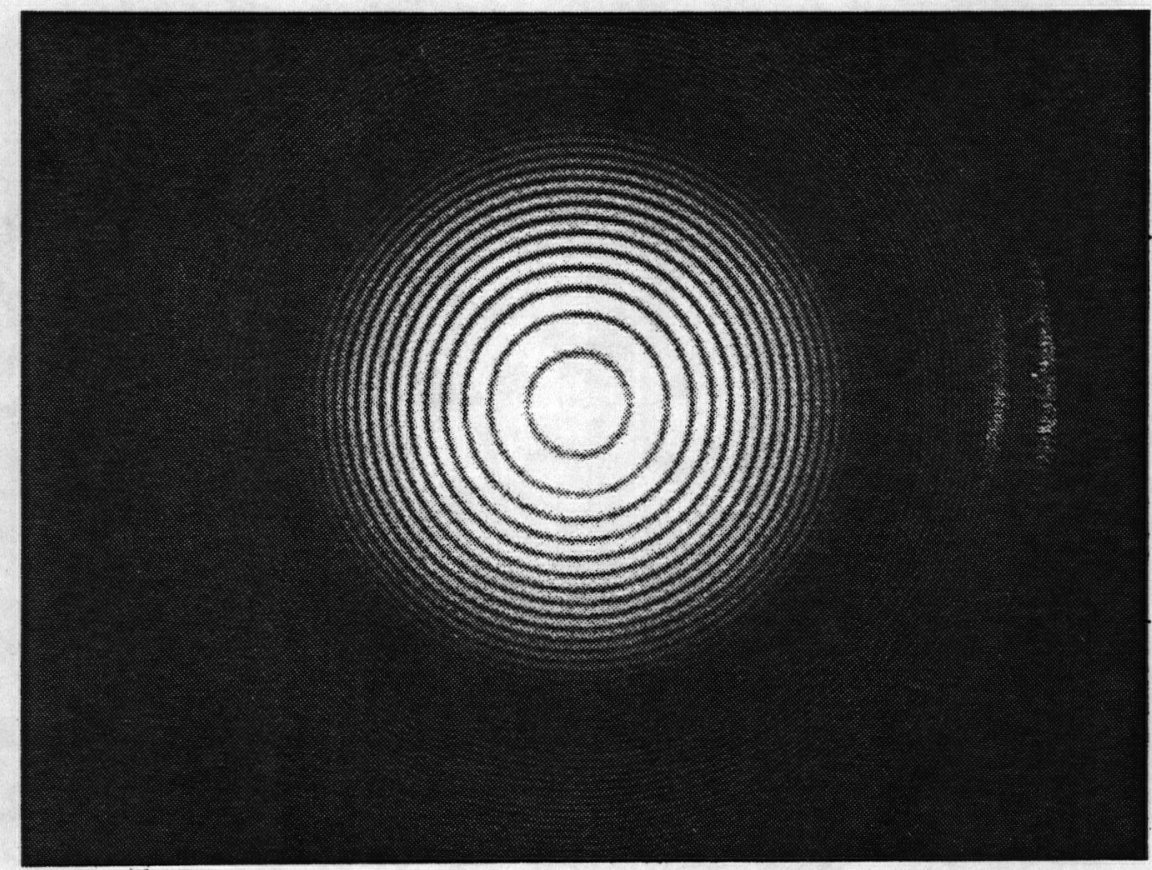

Fig. 2.3. Optical Fourier transform of an in-line hologram of $50 \mu$ glass microspheres. The object-to-hologram separation is $6 \mathrm{~cm}(\mathrm{~N}=$ 12) and $\lambda=5145 \AA$. 


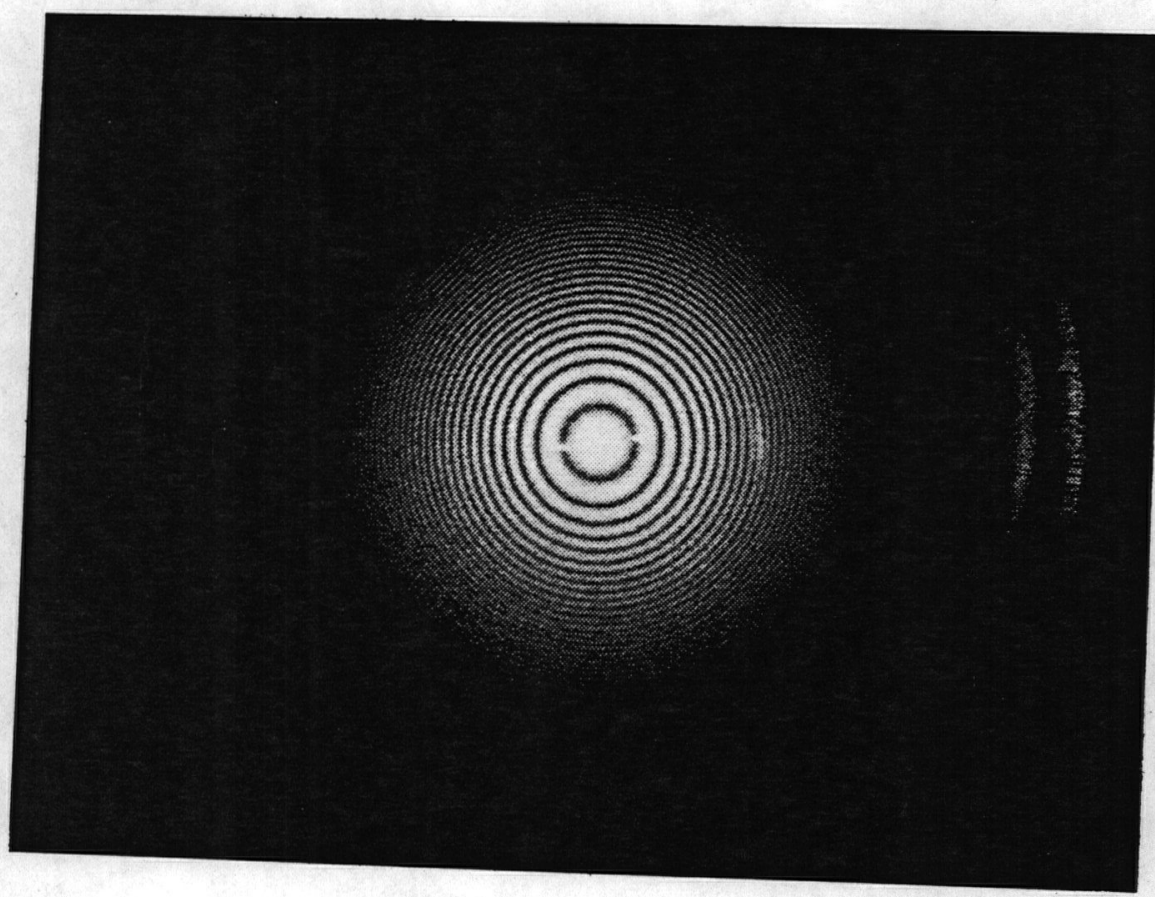

Fig. 2.4. Optical Fourier transform of an in-line hologram of $50 \mu$ glass microspheres. The object-to-hologram separation is $12 \mathrm{~cm}$ ( $N=$ 24) and $\lambda=5145 \AA$. 
$-52-$

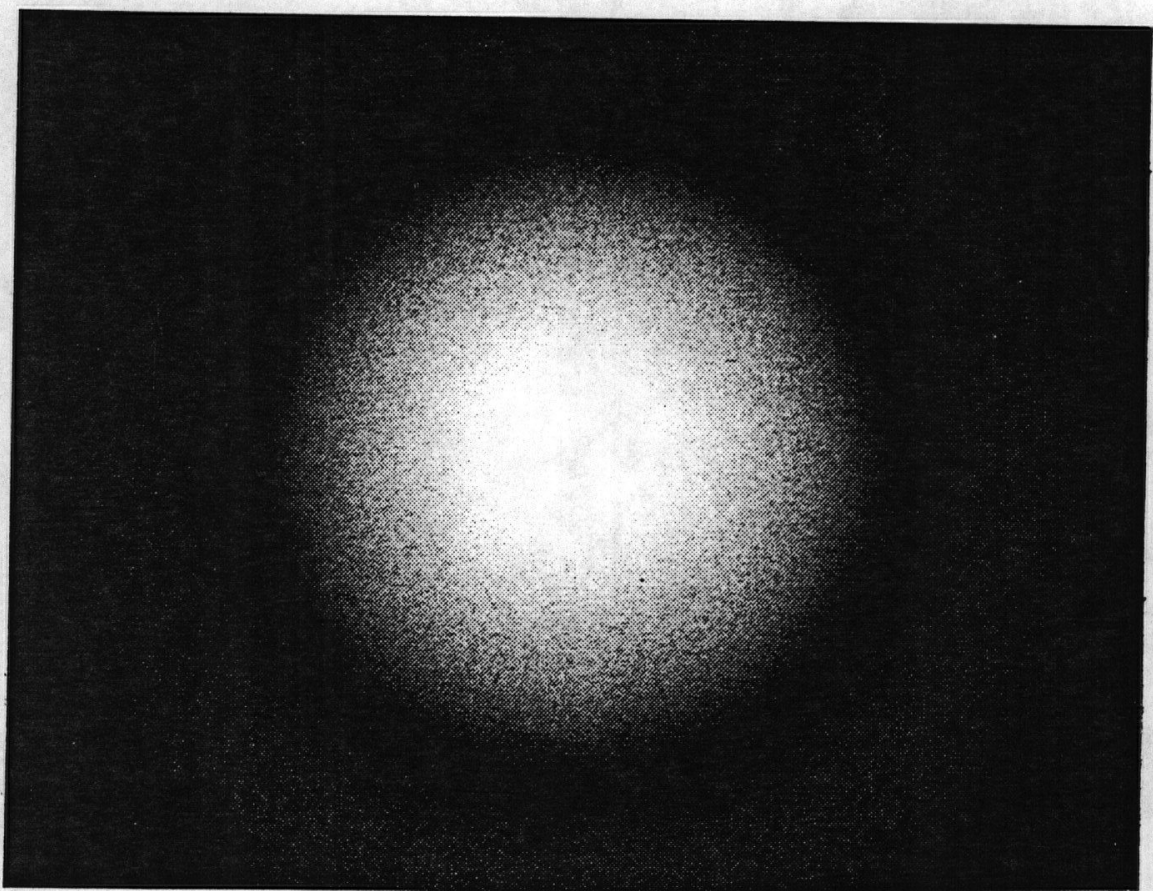

2.5. Total far-field diffraction pattern produced by $50 \mu$ glass microspheres. 
A detailed analysis of speckle is beyond the scope of this thesis; however, two aspects, speckle contrast and speckle size, are quite relevant and will be discussed briefly. The speckle contrast is treated first.

Speckle contrast is defined as the ratio of the standard deviation of intensity to the mean intensity. As mentioned above, the intensity in the transform plane is described by a negative exponential probability density function ( $p d f$ ), explicitly,

$$
p(I)=\frac{1}{2 \sigma^{2}} \exp \left(-\frac{I}{2 \sigma^{2}}\right) \quad \text { for } \quad I>0
$$

and is 0 otherwise. $\sigma^{2}$ denotes the variance of the Gaussian pdf which characterizes the real and imaginary parts of the complex field amplitude (Anderson [1982]). The following moments, which are needed to calculate the speckle contrast, are readily computed by using integration by parts:

$$
\begin{aligned}
& <I>=\int_{0}^{\infty} I p(I) d I=2 \sigma^{2}, \\
& <I^{2}>=\int_{0}^{\infty} I^{2} p(I) d I=8 \sigma^{4}
\end{aligned}
$$

The variance is then obtained by applying the well-known identity, $\sigma_{I}^{2}=\left\langle I^{2}\right\rangle-\langle I\rangle^{2}$; therefore, the speckle contrast is 


$$
C=\frac{\sigma_{I}}{\langle I\rangle}=1 .
$$

The high contrast of the speckle pattern indicates that averaging will be necessary to extract reliable data from measurements of intensity in the transform plane. Depending on the application, temporal and/or spatial averaging may be employed. For particle sizers in which the composite diffraction pattern of a dynamic particulate sample is analyzed, temporal averaging occurs naturally. The moving particles produce a diffraction pattern having an envelope which is stationary in time (the envelope, of course, contains the size information). However, the motion of the particles produces a speckle pattern which is constantly varying, thus, properly choosing the integration time of the detector (or detectors) in the transform plane will average out the speckle effects. If the Fourier transform of a hologram is to be analyzed, the resulting speckle pattern is obviously stationary in time, hence, some type of spatial averaging must be employed. In the simplest situation, the finite active area of a detector (i.e., the pixel size) will locally average the signal, thereby smoothing the measured intensity data. More sophisticated methods of smoothing have also been employed in connection with particle sizing. For example, Stark [1982] and Stark etal. [1975] have treated the smoothing process as a convolution of the actual intensity with a "spectral window" and then proceed to find a window which yields optimum 
results. In any case, the speckle size is clearly important since smoothing must be carried out over an area that is some multiple of the average speckle size.

The "average width" of a speckle is defined as the width of the following correlation function:

$$
R_{I}\left(u_{1}, v_{1} ; u_{2}, v_{2}\right)=\left\langle I_{F}\left(u_{1}, v_{1}\right) I_{F}\left(u_{2}, v_{2}\right)\right\rangle \quad,
$$

where $I_{F}\left(u_{1}, v_{1}\right)$ denotes the intensity in the transform plane at the point $\left(u_{1}, v_{1}\right)$, and similarly, $I_{F}\left(u_{2}, v_{2}\right)$ denotes the intensity at $\left(u_{2}, v_{2}\right)$. The computation is tedious and will not be repeated here (see Goodman [1984]). However, performing the correlation reveals that the average speckle width is of the order of

$$
\delta=\frac{\lambda f}{R}
$$

where $R$ is the radius of the area of illumination of the hologram. As usual, $\lambda$ denotes the wavelength of the illuminating beam and $f$ is the focal length of the transform lens. It is thus observed that the average speckle width is the order of the diameter of the Airy disc of the optical transform system. Furthermore, the speckle size is independent of the number of particles, $N$, provided that $N$ is sufficiently large. 
2.2.4 The transform of a hologram of many particles - case 2: Particles distributed throughout a volume.

The analysis given in Section 2.2 .2 is overly restrictive in at least one respect; namely, the particles were assumed to be located in a plane parallel to the hologram plane. Naturally, in situations of practical interest, the particles are likely to be distributed throughout a volume. Therefore, a more general analysis is required.

The expression describing the field in the transform plane of a hologram of $N$ identical particles confined to a plane was derived in the previous section (see Equation (2.33)) and is rewritten below:

$$
\Psi_{F}(u, v)=\bar{A}\left(\frac{u}{\lambda f}, \frac{v}{\lambda f}\right) \sum_{n=1}^{N} \cos \left(\frac{\pi z \rho^{2}}{\lambda f^{2}}\right) \exp \left|-i 2 \pi\left(u \xi_{n}+u \eta_{n}\right)\right| \ldots
$$

The quantity $z$, denoting the separation between the object and recording planes, is the same for all particles in the sample. However, if the particles are assumed to be distributed throughout a volume, then a unique $z$-coordinate will be associated with each particle in general. In this case, the field in the transform plane of the hologram is

$$
\Psi_{F}(u, v)=\dot{A}\left(\frac{u}{\lambda f}, \frac{v}{\lambda f}\right) \sum_{n=1}^{N} \cos \left(\frac{\pi z_{n} \rho^{2}}{1 f^{2}}\right) \exp \left|-i 2 \pi\left(u \xi_{n}+v \eta_{n}\right)\right|,
$$

where $z_{n}$ denotes the axial distance between the $n^{\text {th }}$ particle and the 
recording plane. The intensity is given by

$$
\begin{aligned}
I_{F}(u, v)=\Psi_{F}(u, v) \Psi_{F}^{*}(u, v) & =\left|\tilde{A}\left(\frac{u}{\lambda f}, \frac{v}{\lambda f}\right)\right|^{2}\left\{\sum_{n=1}^{N} \cos ^{2}\left(\frac{\pi{ }_{n} \rho^{2}}{\lambda f^{2}}\right)\right. \\
& +\sum_{m=1}^{N} \sum_{\substack{m=1 \\
m \neq n}}^{N} \cos \left(\frac{\pi z_{m} \rho^{2}}{\lambda f^{2}}\right) \cos \left(\frac{\pi z_{n} \rho^{2}}{\lambda f^{2}}\right) \\
& \times \exp \left[-i \frac{2 \pi}{\lambda f}\left(u\left(\xi_{m}-\xi_{n}\right)+v\left(\eta_{m}-\eta_{n}\right)\right) \mid\right\}
\end{aligned}
$$

Clearly $I_{F}(u, v)$ is a random variable since it depends upon the random particle coordinates $\left(\xi_{n}, \eta_{n}, z_{n}\right)$ and thus it is appropriate to consider the expected value,

$$
\begin{aligned}
<I_{F}(u, v)>= & \left|\dot{A}\left(\frac{u}{\lambda f}, \frac{v}{\lambda f}\right)\right|^{2}\left\{<\sum_{n=1}^{N} \cos ^{2}\left(\frac{\pi z_{n} \rho^{2}}{\lambda f^{2}}\right)>\right. \\
& +<\sum_{m=1}^{N} \sum_{n=1}^{N} \cos \left(\frac{\pi z_{m} \rho^{2}}{\lambda f^{2}}\right) \cos \left(\frac{\pi z_{n} \rho^{2}}{\lambda f^{2}}\right) \\
& \times \exp \left[-i \frac{2 \pi}{\lambda f}\left(u\left(\xi_{m}-\xi_{n}\right)+v\left(n_{m}-\eta_{n}\right)\right) \mid>\right\}
\end{aligned}
$$

By expressing the cosines in terms of exponentials, the quantity within the double summation may be rewritten as 


$$
\begin{gathered}
\frac{1}{4}\left\{\exp \left[i\left(\phi_{1 m}-\phi_{1 n}\right)\right]+\exp \left[i\left(\phi_{1 m}-\phi_{2 n}\right)\right]+\exp \left[i\left(\phi_{2 m}-\phi_{1 n}\right)\right]\right. \\
\left.+\exp \left[i\left(\phi_{2 m}-\phi_{2 n}\right)\right]\right\}
\end{gathered}
$$

where

$$
\begin{aligned}
& \phi_{1 m}=\frac{\pi z_{m} \rho^{2}}{\lambda f^{2}}-2 \pi\left(u \xi_{m}+v \eta_{m}\right) \quad \text { and } \\
& \phi_{2 m}=-\frac{\pi z_{m} \rho^{2}}{\lambda f^{2}}-2 \pi\left(u \xi_{m}+v \eta_{m}\right)
\end{aligned}
$$

The $\phi_{1 m}$ 's and the $\phi_{2 m}$ 's are assumed to be uniformly distributed in the interval from 0 to $2 \pi$ and statistically independent of one another. Hence, the expected value of the double summation term in Equation (2.45) is identically zero, in analogy with the results leading to Equation (2.37). The average intensity in the transform plane is therefore,

$$
<I_{F}(u, v)>=\left|\tilde{A}\left(\frac{u}{\lambda f}, \frac{v}{\lambda f}\right)\right|^{2}<\sum_{n=1}^{N} \cos ^{2}\left(\frac{\pi z_{n} \rho^{2}}{\lambda f^{2}}\right)>.
$$

It is observed that a cosinusoidal zone lens only appears in the Fraunhofer diffraction pattern when all the $z_{n}$ 's are identical; that is, when all particles are located in a plane. When the particles are distributed throughout a volume, the contributions from the real 
and virtual images of the $n^{\text {th }}$ particle will interfere to produce a zone lens whose frequency is proportional to the distance, $z_{n}$. As a result, the total diffraction pattern is composed of an envelope, $|\tilde{A}(\cdot)|^{2}$, modulating a sum of zone lens terms, each having a different frequency.

Without any knowledge of the statistics of the $z_{n}^{\prime} s$, the expected value of Equation (2.47) cannot be evaluated. In many situations encountered in practice, however, it is reasonable to assume that the $z_{n}$ 's are independent random variables described by a common probability density function, $f(z)$. If so, Equation (2.47) may be rewritten as

$$
\left\langle I_{F}(u, v)\right\rangle=N\left|\dot{A}\left(\frac{u}{\lambda f}, \frac{v}{\lambda f}\right)\right|^{2} \int_{-\infty}^{+\infty} f(z) \cos ^{2}\left(\frac{\pi z \rho^{2}}{\lambda f^{2}}\right) d z
$$

The above equation indicates that the Fraunhofer diffraction pattern of an in-line hologram contains information characteristic of the manner in which the particles are distributed along the $z$-axis, in addition to particulate size information.

In many cases of interest, the particles to be studied are located in a chamber or a cell and thus the boundaries of the sample volume are well-defined. As an example, it is asumed that the particles are located between two planes, $z=z_{1}$ and $z=z_{2}$ (see Fig. 2.6) and are uniformly distributed in the interval $z_{2}<z<z_{1}$, i.e., 


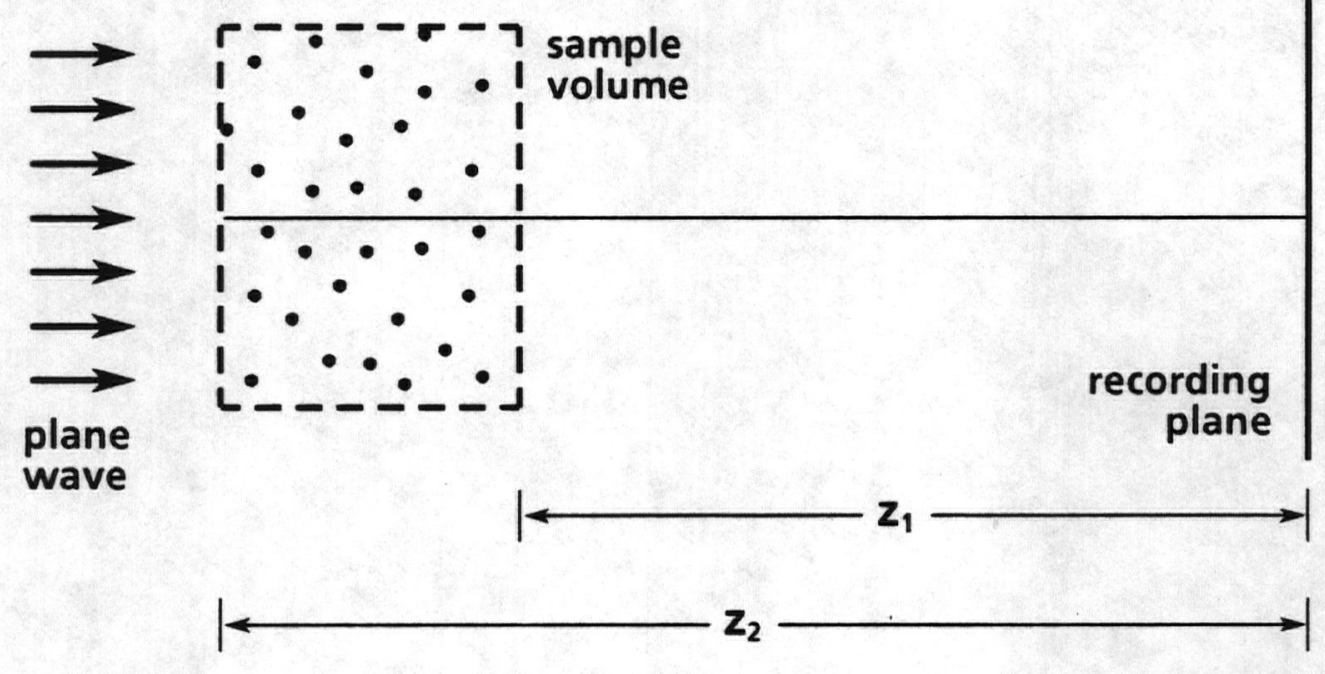

Fig. 2.6. Hologram recording geometry. The particles are assumed to be uniformly distributed within the interval $z_{1}<z<z_{2}$. 


$$
f(z)=\left\{\begin{array}{cl}
1 /\left(z_{2}-z_{1}\right) & \text { for } z_{2}<z<z_{1} \\
0 & \text { otherwise }
\end{array} .\right.
$$

Substituting the above expression into Equation (2.48) yields

$$
\begin{aligned}
<I_{F}(u, v)> & =N\left|\tilde{A}\left(\frac{u}{\lambda f}, \frac{v}{\lambda f}\right)\right|^{2} \\
& \times\left\{\frac{1}{2}+\frac{1}{2\left(z_{2}-z_{1}\right)} \frac{\lambda f^{2}}{2 \pi \rho^{2}}\left[\sin \left(\frac{2 \pi z_{2} \rho^{2}}{\lambda f^{2}}\right)-\sin \left(\frac{2 \pi z_{1} \rho^{2}}{\lambda f^{2}}\right) \mid\right\} .\right.
\end{aligned}
$$

Applying the trigonometric identity,

$$
\sin a-\sin \beta=2 \cos \frac{a+\beta}{2} \sin \frac{a-\beta}{2},
$$

yields

$$
<I_{F}(u, v)>=I_{0}(u, v)\left\{\frac{1}{2}+\frac{1}{2} \cos \left(\frac{2 \pi \rho^{2}}{\lambda f^{2}} \frac{\left(z_{2}+z_{1}\right)}{2}\right) \operatorname{sinc}\left(\frac{2 \rho^{2}}{\lambda f^{2}} \frac{\left(z_{2}-z_{1}\right)}{2}\right)\right\},
$$

where

$$
I_{0}(u, v)=N\left|\bar{A}\left(\frac{u}{\lambda f}, \frac{v}{\lambda f}\right)\right|^{2}
$$

and $\operatorname{sinc}(x) \equiv \sin (\pi x) /(\pi x)$. As expected, the quantity in braces (i.e., $\left.\left\langle I_{F}(u, v)\right\rangle / I_{v}(u, v)\right)$ describes the transmittance of a zone lens only when the sample depth, $z_{2}-z_{i}$, approaches zero. When the sample 
depth is non-zero, the cosine in the above expression is multiplied by a sinc function whose characteristic width is inversely related to the sample depth. To illustrate, the quantity $\left\langle I_{F}(u, v)>/ I_{0}(u, v)\right.$ is plotted versus the radial coordinate, $\rho$, for two different sets of values of $z_{1}$ and $z_{2}$. Figure 2.7 a corresponds to using $z_{1}=4.0 \mathrm{~cm}$ and $z_{2}=7.8 \mathrm{~cm}$; Fig. $2.7 \mathrm{~b}$ corresponds to using $z_{1}=z_{2}=5.9 \mathrm{~cm}$. In obtaining both plots, the values used for the wavelength and for the focal length of the transform lens are $\lambda=5145 \AA$ and $f=0.5 \mathrm{~m}$, respectively. It should be noted that $\left(z_{1}+z_{2}\right) / 2$ is the same in both cases; therefore, the only difference between the quantities plotted is the argument of the sinc function, which is directly related to the depth of the sample volume.

To experimentally demonstrate the effect of sample depth on the transform pattern, a hologram of particles contained in a cubeshaped volume was recorded. The sample volume consisted of a cell, made of $5 \mathrm{~cm} \times 5 \mathrm{~cm}$ glass plates, which was filled with distilled water. Polystyrene microspheres $(52.8 \mu$ in diameter) were then added to the water to produce a number density of roughly $10^{3}$ particles per $\mathrm{cm}^{3}$. To distribute the particles as uniformly as possible, the mixture was agitated and then allowed to sit momentarily until the swirling motion of the water ceased. The hologram was then recorded using a cw Argon-ion laser operating at $5145 \AA$. The resulting optical Fourier transform of the hologram is shown in Fig. 2.8. 

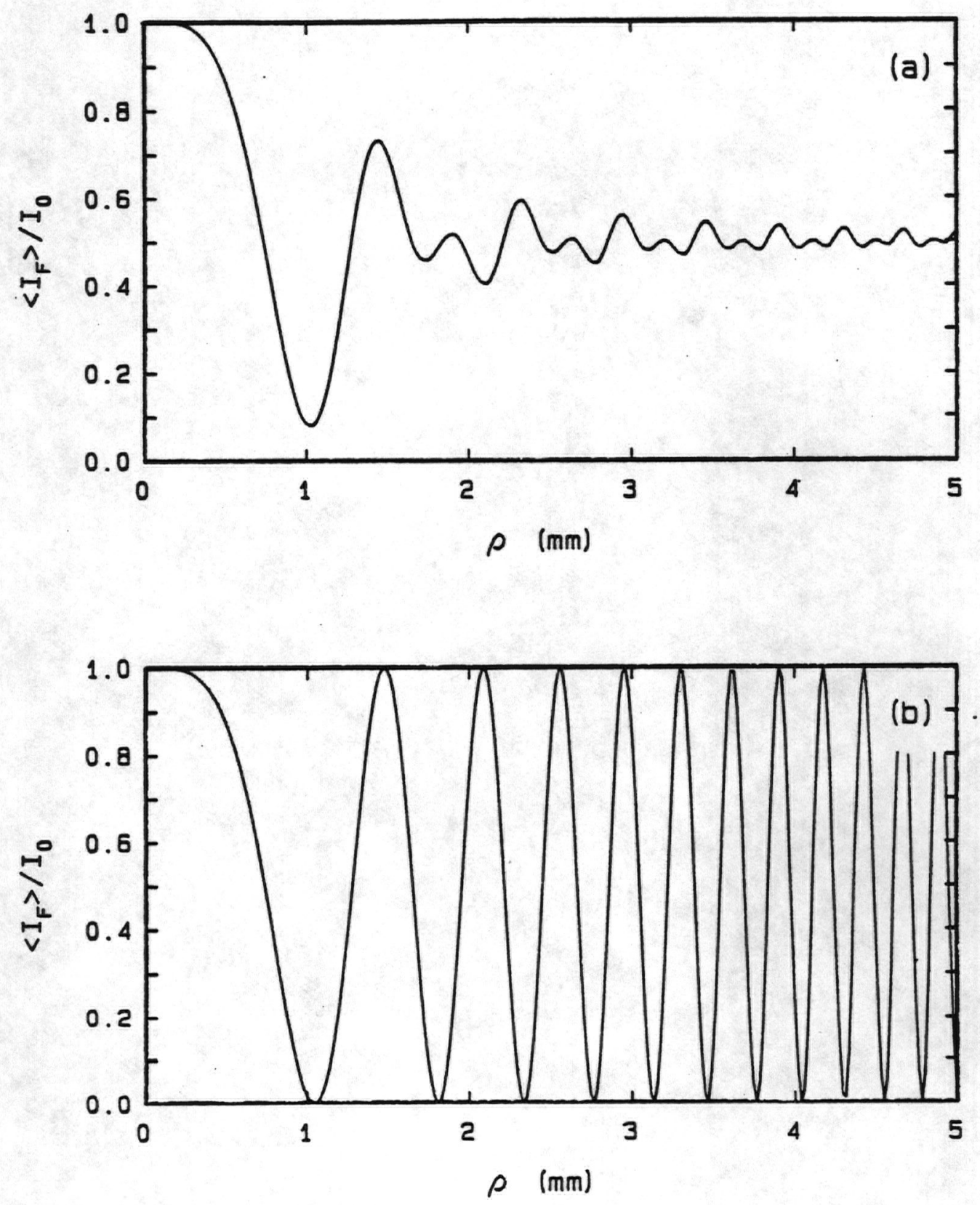

Fig. 2.7. Illustrating the $z$-dependent structure in the transform plane of an in-line far-field hologram. (a) Plot of $\left\langle I_{F}(u, v)\right\rangle / I_{0}(u, v)$ vs. the radial coordinate $\rho$ for $l=514.5 \mathrm{~nm}, f=0.5 \mathrm{~m}, z_{1}=4.0 \mathrm{~cm}$ and $z_{2}=7.8 \mathrm{~cm}$. (b) Plot of $\left\langle I_{F}(u, v)\right\rangle / I_{0}(u, v)$ vs. the radial coordinate $\rho$ for $\lambda=514.5 \mathrm{~nm}, f=0.5 \mathrm{~m}, z_{1}=z_{2}=5.9 \mathrm{~cm}$. (See Equation (2.52).) 


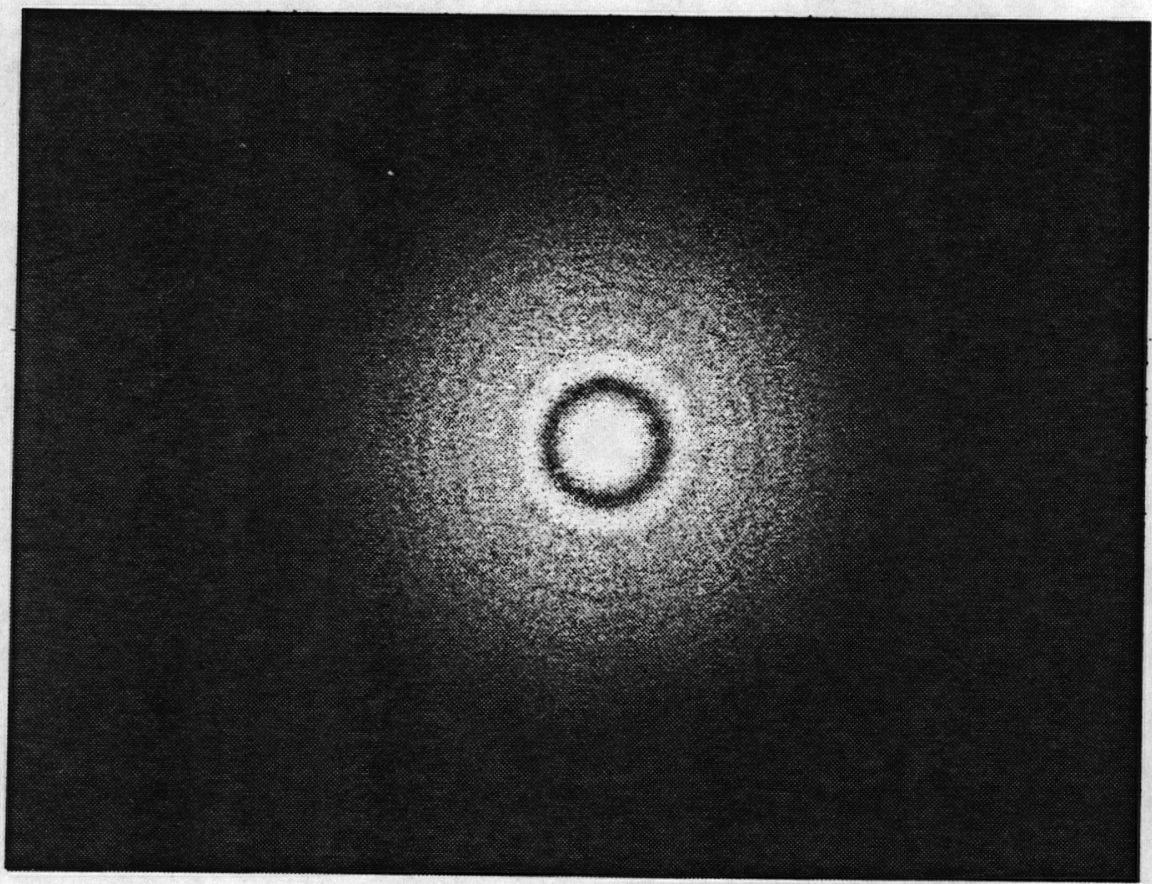

Fig. 2.8. The optical Fourier transform of $52.8 \mu$ polysytrene spheres suspended in water. The physical depth of the sample was $5 \mathrm{~cm}$. 
At this point, a brief digression is appropriate in order to discuss the relationship between the physical depth of the sample volume compared to the apparent depth as measured from the holographic reconstruction. In reference to Fig. 2.9, the depth of the sample is denoted by $z_{0}$ and the separation between the sample and the recording medium is given by $z_{1}$. If the hologram is played back, it is observed that the depth of the image of the sample volume is smaller than that of the original volume by a factor of $1 / n$ (where $n$ is the refractive index of the liquid in the sample volume). This result may be obtained by two successive applications of the Huygens-Fresnel Principle: first, to describe the wave propagation from the object plane to the boundary and second, to describe the propagation from the boundary to the recording plane. If a single particle, described by $A(\xi, \eta)$ is considered, then the complex field amplitude at the boundary is

$$
\begin{aligned}
\Psi_{B}\left(\xi^{\prime}, \eta^{\prime}\right) & =-i \frac{n}{\lambda z_{0}} \exp \left(i k n z_{0}\right) \\
& \times \iint_{-\infty}^{+\infty}[1-A(\xi, \eta)] \exp \left\{i \frac{k n}{2 z_{0}}\left[\left(\xi^{\prime}-\xi\right)^{2}+\left(\eta^{\prime}-\eta\right)^{2}\right]\right\} d \xi d \eta,
\end{aligned}
$$

where $\xi^{\prime}$ and $\eta^{\prime}$ are the coordinates in the boundary plane (see Fig. 2.9). Furthermore, $k$ denotes the propagation constant in free space and thus $k n$ denotes the propagation constant in a medium with a refractive index of $n$. The second application of the HuygensFresnel Principle gives the field in the recording plane, i.e., 


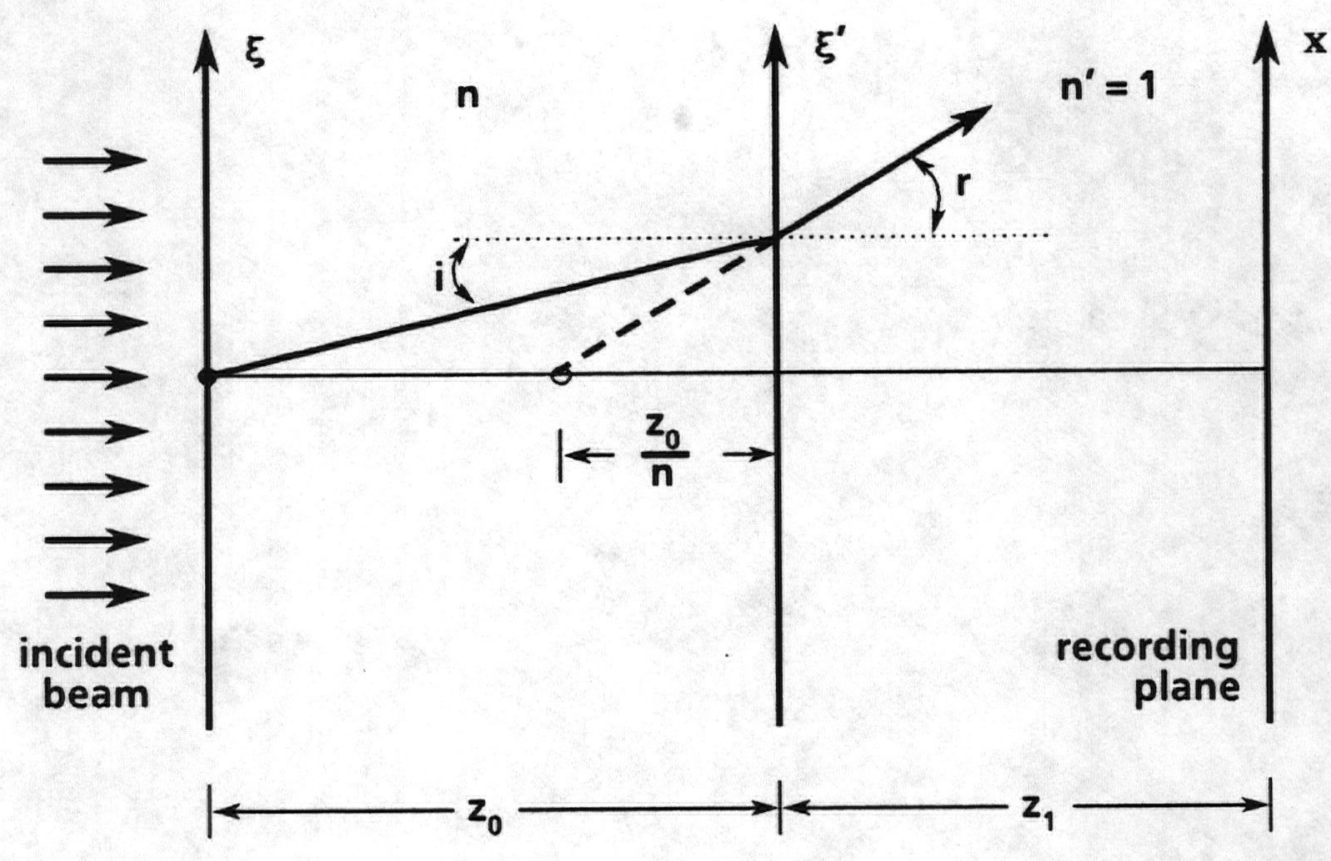

Fig. 2.9. Illustrating the apparent sample volume depth in an inline hologram. The sample volume has length $z_{0}$ and index of refraction $n$; the air gap between the sample and the recording plane has length $z_{1}$. Applying Snell's law in paraxial form ( $n i=n^{\prime} r$ ) reveals that a particle located at a distance $z_{0}$ from the interface appears to be located at $z_{0} / n$. 


$$
\begin{aligned}
& \Psi_{H}(x, y)=-i \frac{1}{\lambda z_{1}} \exp \left(i k z_{1}\right) \\
& \quad \times \iint_{-\infty}^{+\infty} \Psi_{B}\left(\xi^{\prime}, \eta^{\prime}\right) \exp \left\{i \frac{k}{2 z_{1}}\left[\left(x-\xi^{\prime}\right)^{2}+\left(y-\eta^{\prime}\right)^{2}\right]\right\} d \xi d \eta^{\prime}
\end{aligned}
$$

Substituting Equation (2.53) into (2.54) and performing the integration with respect to the primed coordinates yields

$$
\begin{aligned}
& \Psi_{H}(x, y)=-i \frac{1}{\lambda\left(\frac{z_{0}}{n}+z_{1}\right)} \exp \left[i k\left(n z_{0}+z_{1}\right)\right] \\
& \quad \times \iint_{-\infty}^{+\infty}[1-A(\xi, \eta)] \exp \left\{i \frac{k}{2\left(\frac{z_{0}}{n}+z_{1}\right)}\left[(x-\xi)^{2}+(y-\eta)^{2}\right]\right\} d \xi d \eta
\end{aligned}
$$

Upon comparing Equations (2.55) and (2.1), it is observed that the former may be interpreted as the complex field amplitude resulting from an object located at a distance $z_{0} / n+z_{1}$ from the recording plane. Thus, a sample volume having a physical depth of $z_{0}$ would appear to have a depth of $z_{0} / n$ in the holographic image.

Alternatively, a geometrical optics approach may be used to provide an intuitive explanation of the reduced sample depth. If a typical particle in the sample volume is treated as a point scatterer, then the scattered 1 ight is clearly a spherical wave and is easily handled by ray-tracing. By applying Snell's law (in 
paraxial form) to a "scattered ray", it is observed from Fig. 2.9 that a particle located at a point $z_{0}+z_{1}$ from the recording plane appears to be located at the point $z_{0} / n+z_{1}$.

Returning to the main theme, it will be recalled that a sample depth of $5 \mathrm{~cm}$ was used in recording the hologram from which the optical Fourier transform, shown in Fig. 2.8, was obtained. Since the particles were suspended in water having a refractive index of roughly 1.33 , the apparent sample depth is $3.8 \mathrm{~cm}$ which corresponds to the depth used in obtaining the plot shown in Fig. 2.7a. In addition, the wavelength and focal length parameters used to produce Fig. 2.7a are the same as those used in forming the optical transform of the actual hologram. Therefore, the plot shown in Fig. 2.7a corresponds to the transform pattern in Fig. 2.8 in a quantitative sense. However, the envelope function, $|\tilde{A}(\cdot)|^{2}$ in Equation (2.52) was not included in this plot in order to emphasize the effects of the sample depth; it will now be included to allow a more complete comparison between theory and experiment. For a spherical particle of radius $a$, the object function is given by

$$
A(\xi, \eta)=\operatorname{circ} \frac{\sqrt{\xi^{2}+\eta^{2}}}{a} .
$$

The corresponding Fourier transform is (Goodman [1968]) 


$$
\overline{\mathrm{A}}\left(\frac{u}{\lambda f}, \frac{v}{\lambda f}\right)=\pi a^{2}\left[\frac{2 J_{1}\left(\frac{2 \pi a \rho}{\lambda f}\right)}{\frac{2 \pi a \rho}{\lambda f}}\right],
$$

where $\rho^{2}=u^{2}+v^{2}$ and $J_{1}$ is the Bessel function of the first kind and of order one. Substituting the above expression into Equation (2.52) yields

$$
\begin{aligned}
<I_{F}(\rho)> & =N \pi^{2} a^{4}\left[\frac{2 J_{1}\left(\frac{2 \pi a \rho}{\lambda f}\right)}{\frac{2 \pi a \rho}{\lambda f}}\right]^{2} \\
& \times\left\{\frac{1}{2}+\frac{1}{2} \cos \left(\frac{2 \pi \rho^{2}}{\lambda f^{2}} \frac{\left(z_{2}+z_{1}\right)}{2}\right) \operatorname{sinc}\left(\frac{2 \rho^{2}}{\lambda f^{2}} \frac{\left(z_{2}-z_{1}\right)}{2}\right)\right\} .
\end{aligned}
$$

A plot of $\left\langle I_{F}(\rho)>\right.$ vs. $\rho$ is given in Fig. 2.10. In obtaining the plot, the parameter $2 a$ was assigned a value of $52.8 \mu$, corresponding to the diameter of the particles used in the actual experiment. The remaining parameters $\left(\lambda, f, z_{1}\right.$ and $\left.z_{2}\right)$ have the same values as those used in obtaining Fig. 2.7a.

The intensity profile of the optical transform shown in Fig. 2.8 is plotted against radial position in Fig. 2.11. The data for this plot was obtained by placing a TV camera in the transform plane of the hologram and using an image digitizer to scan the pattern along a radius. The intensity profile shown in Fig. 2.11 is noisy due to speckle. Hence, it is necessary to average several scans 
taken along different radii before comparing the theoretical result to the experimental result. The average of 25 such scans is shown in Fig. 2.12 and it is observed that the random fluctuations have been reduced considerably. (For comparison, a plot of the theoretical intensity profile has been included in Fig. 2.12).

On comparing the two curves in Fig. 2.12, it is observed that there is good agreement between theory and experiment. The most distinguishing feature, the minimum of intensity near $\rho=1 \mathrm{~mm}$ is presesnt in both plots. Some discrepancies are observed, especially for $\rho>2.0 \mathrm{~mm}$, and are probably due to the assumption that the particles are uniformly distributed throughout the sample volume. The actual distribution is undoubtedly more complicated. Nonetheless, the simplified model based on the uniform distribution is quite useful in that it gives a good indication of how sample depth affects the intensity pattern in the transform plane.

2.2.5 The effects of random particle orientations and particle-size distributions in the Fourier plane

The analysis up to this point has been limited to a rather specific class of particle field: one in which all particles were assumed to be identical in size, shape and orientation. However, the intent was to illustrate similarities and differences between the total diffraction pattern of a particle sample compared to the Fourier transform of a hologram of the same sample. Clearly, the above assumptions allowed for the comparison in a straightforward manner. A more general analysis, based on theory developed for 


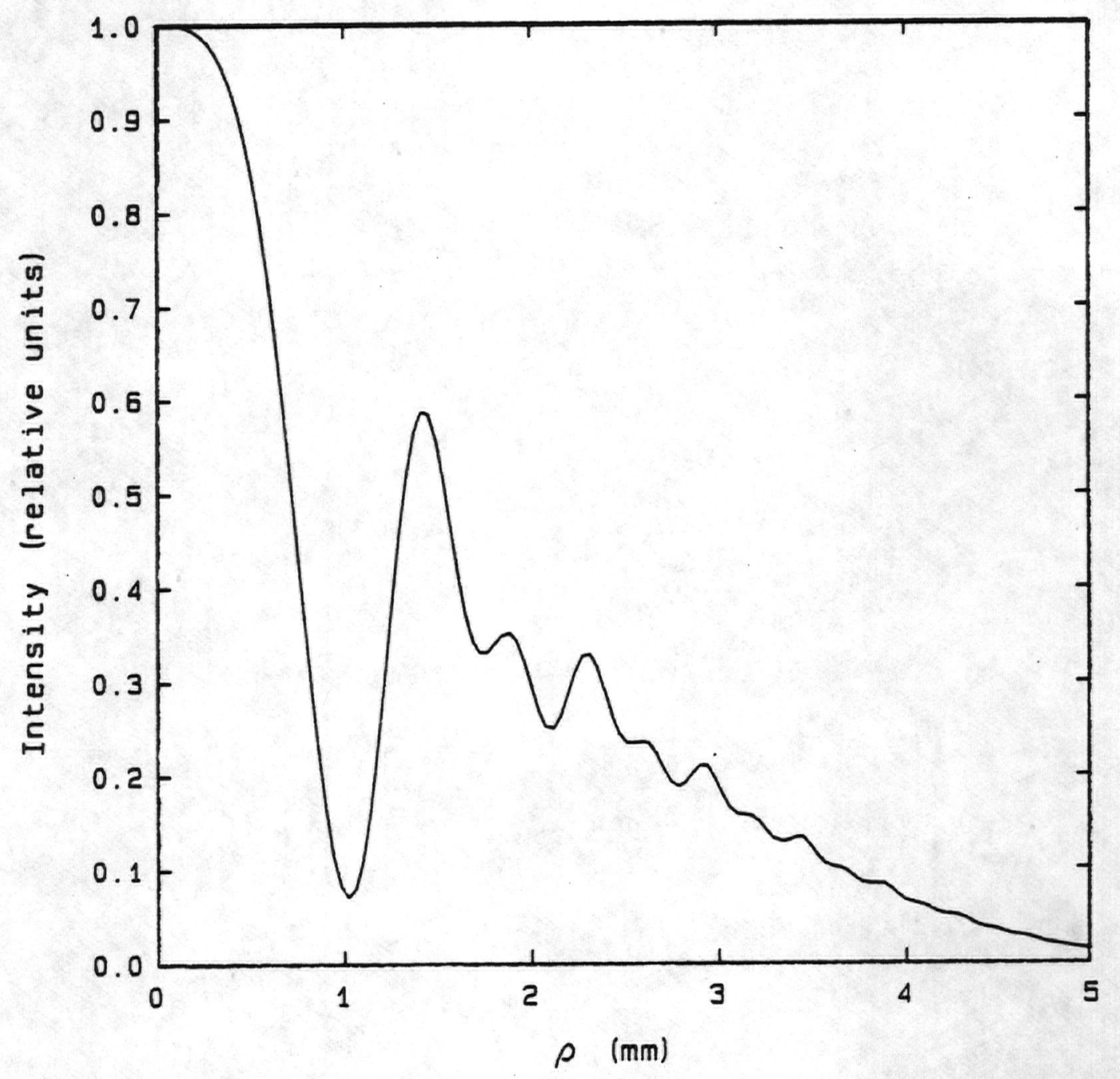

Fig. 2.10. Theoretical intensity profile in the Fourier transform plane of an in-line hologram of $52.8 \mu$ particles. The particles were assumed to be uniformly distributed along the axial interval, $4.0 \mathrm{~cm}<z<7.8 \mathrm{~cm}$. 


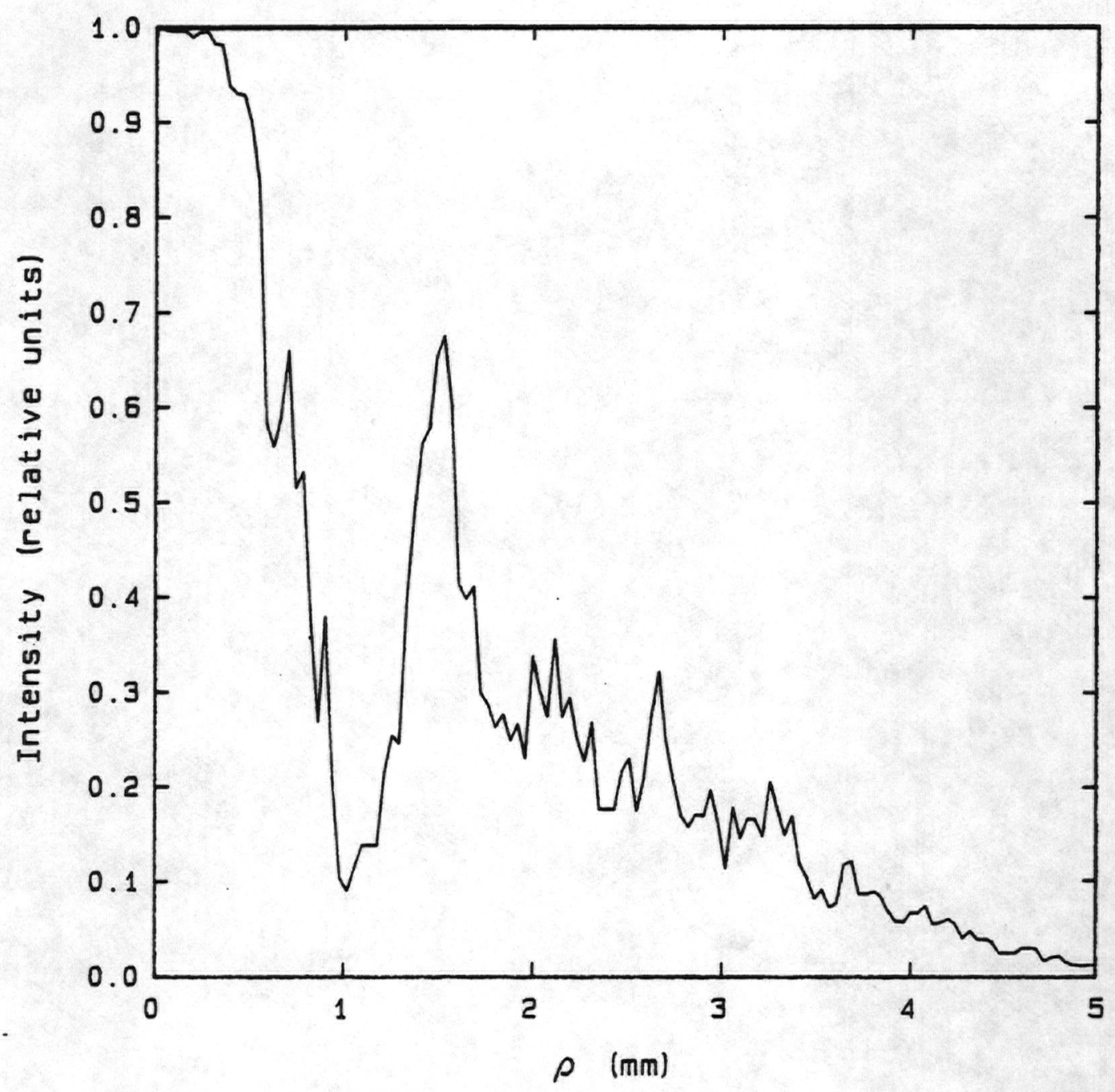

Fig. 2.11. Measured intensity profile resulting from a single scan along a radius in the optical Fourier transform plane of an in-line hologram of $52.8 \mu$ particles. The particles were distributed along the axial interval, $4.0 \mathrm{~cm}<z<7.8 \mathrm{~cm}$. 


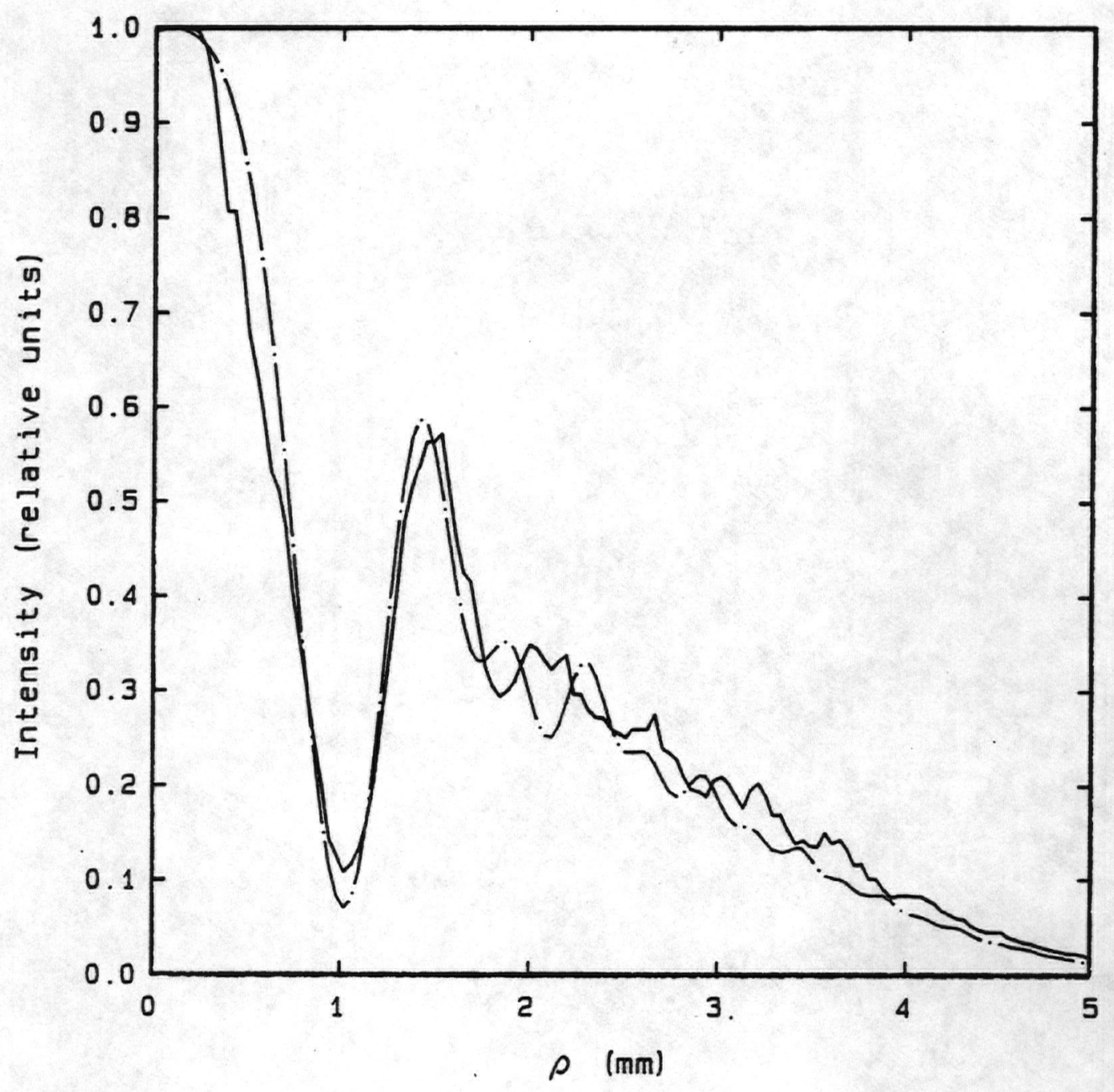

Fig. 2.12. Same as Fig. 2.11, except 25 radial scans were averaged to reduce the fluctuations due to speckle. 
sizing and counting particles from the total diffraction pattern, is presented in this section.

Anderson and Beissner [1971] have studied the intensity pattern in the Fourier transform plane of a collection of particles which are randomly oriented and have different sizes. Assuming for the moment that the orientations are random but the particles are otherwise identical, Anderson and Beissner have shown that the expected value of the intensity in the transform plane is

$$
\langle I(\rho)\rangle=N \int_{0}^{2 \pi}|\tilde{A}(\rho, \phi)|^{2} d \phi \quad,
$$

where $\tilde{A}(\rho, \phi)$ is the Fourier transform of the object function, $\tilde{A}(\xi, \eta)$, expressed in polar coordinates. $N$, of course, is the number of particles in the sample. The above result indicates that the averaged intensity is proportional to the angular average of the intensity pattern produced by a single particle, and hence, it is independent of the polar angle $\phi$. That is, the far-field diffraction pattern of a single particle is, in general, $\phi-$ dependent, but the random orientations of the particles comprising the sample will cause the angular dependence to average out in the total diffraction pattern.

To allow for different sizes and shapes, the particles are divided into $M$ classes or subpopulations. Random orientations are allowed but the particles are otherwise assumed to be identical within a given class. If a typical particle from the $j^{\text {th }}$ class is 
described by the object function $A_{j}(\xi, \eta)$, the resulting expression for the intensity in the transform plane is (Anderson and Beissner [19711)

$$
\left\langle I(\rho)>=\sum_{j=1}^{M} N_{j}<G_{j}(\rho)>\right.
$$

where

$$
\left\langle G_{j}(\rho)\right\rangle=\int_{0}^{2 \pi}\left|\tilde{A}_{j}(\rho, \phi)\right|^{2} d \phi \quad .
$$

and $N_{j}$ denotes the number of particles belonging to the $j^{\text {th }}$ class. Thus, the resulting intensity pattern is the sum of the intensity patterns produced by the individual subpopulations.

The above equations may be combined with the results from Sections 2.2 .2 and 2.2.4 to describe the intensity in the transform plane of a hologram of a polydisperse field of particles. For example, the average intensity in the transform plane of a hologram of $N$ identical particles is given by Equation (2.37) which is rewritten below:

$$
<I_{F}(u, v)>=N\left|\tilde{A}\left(\frac{u}{\lambda f}, \frac{v}{\lambda f}\right)\right|^{2} \cos ^{2}\left(\frac{\pi z \rho^{2}}{\lambda f^{2}}\right) .
$$

For $M$ classes of particles, the corresponding expression would be 


$$
<I_{F}(\rho)>=\cos ^{2}\left(\frac{\pi z \rho^{2}}{\lambda f^{2}}\right) \sum_{j=1}^{M} N_{j} G_{j}(\rho)
$$

On comparing Equations (2.60a) and (2.61), it is observed that the only difference is the zone lens term which results when forming the Fourier transform of an in-line hologram. Since the above expression accounts for particles of different sizes, it can be of value in developing a hologram-based sizing instrument.

\subsection{The Fourier transform of the reconstructed image of a singly-exposed off-axis} hologram

It was shown in Section 2.2 that, in addition to particulate size information, the transform pattern produced by an in-line hologram contains structure that is dependent on the manner in which the particles are distributed along the $z$-axis. If the particles are all located in a plane prior to recording the hologram, this structure is just a cosinusoidal zone lens. In some cases, it may be necessary to remove the zone lens. For example, it is necessary to remove the zone lens when attempting to measure particle velocities from intensity data obtained in the transform plane of a double-exposure hologram (see Chapter 3 ). Since a detector having finite resolution will invariably be used in the transform plane, increasing the spatial frequency of the zone lens beyond the resolution limit of the detector will effectively eliminate the zone lens. Hence, properly choosing the recording distance will serve to eliminate the zone lens since its spatial frequency is directly 
proportional to the recording distance (i.e., the quantity $z$ in Equation 2.37).

Alternatively, off-axis holography may be used since the zone lens in the in-line case results from interference of the fields corresponding to. the real and virtual images. In a properly recorded off-axis hologram, the real and virtual images are located on separate axes, thereby prohibiting any interaction between them. Therefore, a zone lens does not appear in the transform plane.

\subsubsection{The off-axis hologram and the Fourier transform of the reconstructed image} assuming linear recording

A variety of recording geometries are possible with off-axis holography, however, the configuration shown in Fig. 2.13a is wellsuited for Fourier plane analysis of the reconstructed image. The recording geometry shown is equivalent to the in-line configuration (Fig 2.1a) combined with an off-axis reference wave. In recording the hologram, a collimated beam is used to illuminate the object and a second, off-axis collimated beam forms the reference wave. Some workers using off-axis holography for studying particulates have chosen to illuminate the sample with diffuse light (see, for example, Haussmann and Lauterborn [1980] and Trolinger [1975]). In such cases, a ground glass diffuser would be placed between the direct laser beam and the sample, producing diffuse illumination which subsequently makes the reconstructed image easier to view with the unaided eye. However, non-diffuse illumination is clearly 


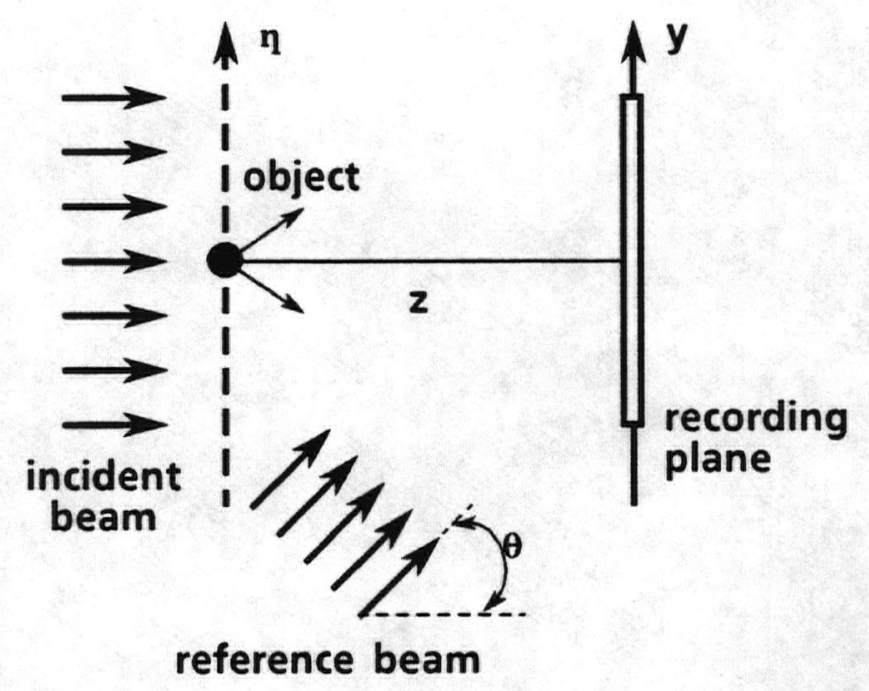

(a)

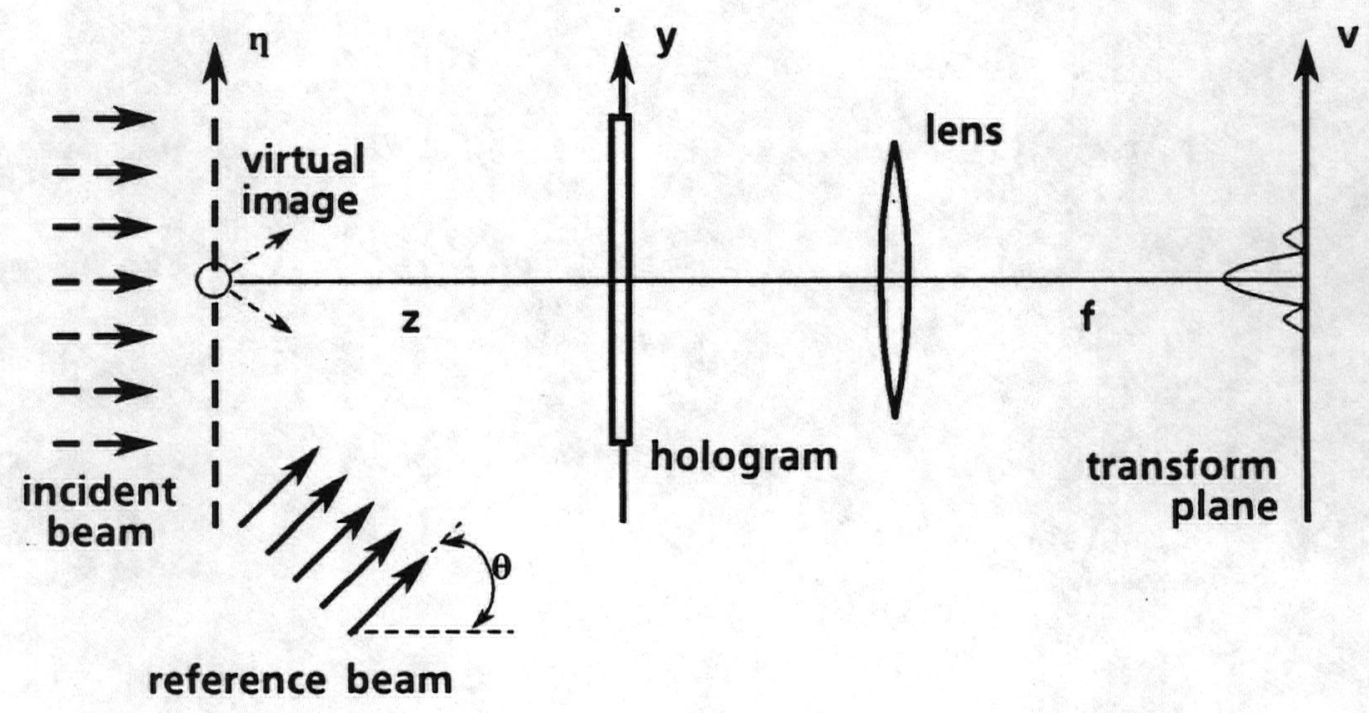

(b)

Fig. 2.13. (a) Recording geometry for off-axis holography. (b) Image reconstruction and Fourier transformation. 
preferable when a Fourier plane analysis of the reconstructed image is to be performed.

Assuming that a collimated beam is used to illuminate the sample, the object wave will consist of two components: (1) the diffracted light produced when the incident beam interacts with the object and (2), the undiffracted portion of the incident beam. The reference wave will, of course, interfere with the object wave and the resulting interference pattern is recorded, yielding a hologram. If the intensity pattern is recorded linearly, re-illuminating the hologram with the original reference beam will reproduce the original object wave. Thus, forming the Fourier transform of the reconstructed image (see Fig. 2.13b) is equivalent to obtaining the transform of the original object. This is illustrated in Fig. 2.14 which shows a photograph of the intensity pattern in the transform plane of the reconstructed image from an off-axis hologram. The particle sample used in recording the hologram was similar to that used in previous experiments, namely, $50 \mu$ glass spheres distributed on a $5 \mathrm{~cm} \times 5 \mathrm{~cm}$ glass slide. As expected, the transform pattern is indistinguishable from the total diffraction pattern produced directly by a similar particle sample (cf. Fig. 2.5). However, it is shown in the following section that if the hologram is recorded nonlinearly, false images are produced in the reconstruction which result in a zone lens in the transform plane. 


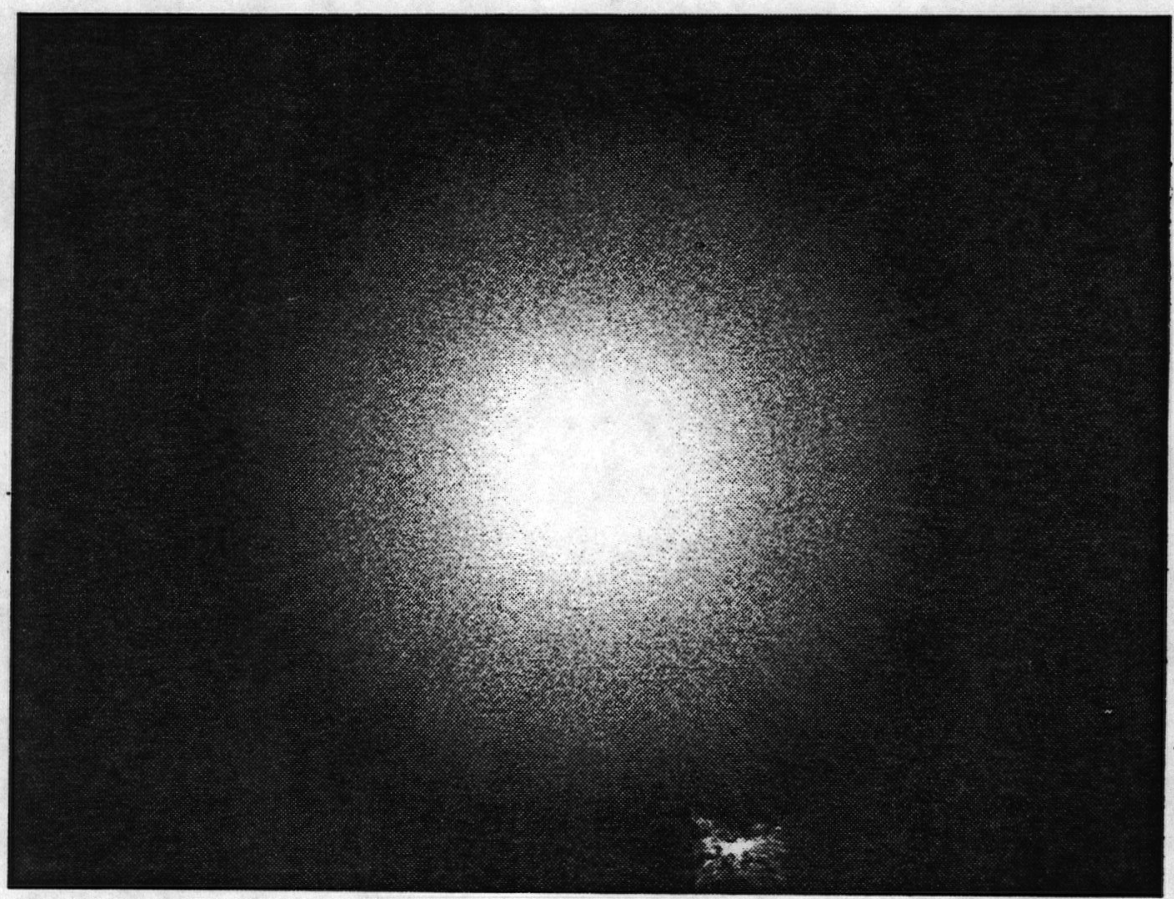

Fig. 2.14. The intensity pattern in the transform plane of an offaxis hologram. $50 \mu$ glass microspheres were used in recording the hologram. 


\subsubsection{The off-axis hologram assuming nonlinear recording}

The effects of film nonlinearities in holography haved been examined by a number of authors (see, for example, Kozma [1968], Bryngdahl and Lohmann [1968] and the references contained therein). One result of nonlinear recording is the production of false images which may exist at various locations with respect to the real and virtual images. It is difficult to generalize as to the exact nature and location of the false images since they are dependent on a number of factors including the recording geometry, the illumination (diffuse or non-diffuse) and the properties of the object itself. Given these parameters, however, the properties of the false images may be obtained by choosing a suitable model for the recording nonlinearity and performing the appropriate calculations.

In reference to Figs. $2.13 a$ and $b$, it is observed that a linearly recorded hologram of an object at a distance $z$ to the left of the recording plane produces a virtual image (at a distance $z$ to the left of the hologram plane) when the hologram is illuminated with the original reference wave. Of course, the undiffracted portion of the incident beam used to illuminate the object is also reproduced. As mentioned, nonlinear recording can lead to a series of false images. Of major concern is the false image which occurs at a distance $z$ to the right of the hologram plane, in line with the virtual image. When this occurs, the reconstructed images are 
similar to those produced by an in-line hologram and as a result, a zone lens is produced in the transform plane. At this point, it is not obvious (from the results given by other authors) that such a false image will occur; nevertheless, it has been observed in practice and the experimental results are presented later in this section. First, however, a theoretical explanation is given.

To begin the analysis, the complex field amplitude in the hologram plane must be obtained. The reference wave in the recording plane is denoted by $r(x, y)$ where

$$
r(x, y)=r_{0} \exp [i k y \sin \theta],
$$

and $\theta$ denotes the angle of propagation (see Fig. 2.13a). The object wave in the recording plane is determined by the Huygens-Fresnel Principle and is given by

$$
\begin{aligned}
a(x, y)= & -i \frac{a_{0}}{\lambda z} \exp [i k z] \\
& \times \iint_{-\infty}^{+\infty}[1-A(\xi, \eta)] \exp \left\{i \frac{k}{2 z}\left[(x-\xi)^{2}+(y-\eta)^{2}\right]\right\} d \xi d \eta
\end{aligned}
$$

where $\xi$ and $\eta$ denote the coordinates in the object plane, $x$ and $y$ denote the coordinates in the hologram plane and $A(\xi, \eta)$ describes the object's cross-sectional shape. In addition, $a_{0}$ represents the amplitude of the plane wave incident on the object. It is observed that the object wave for the off-axis hologram is given by the same expression that describes the complex field distribution in the 
recording plane of an in-1ine hologram (cf. Equation (2.3)) because the recording geometries are similar in both cases. After a straightforward integration, Equation (2.63) may be rewritten to express the object wave in terms of diffracted and undiffracted components, i.e.,

$$
a(x, y)=a_{0} \exp [i k z]\left[1+\frac{i}{\lambda z} \bar{A}(x, y)\right],
$$

where

$$
\bar{A}(x, y)=\iint_{-\infty}^{+\infty} A(\xi, \eta) \exp \left\{i \frac{\pi}{\lambda z}\left[(x-\xi)^{2}+(y-\eta)^{2}\right]\right\} d \xi d \eta
$$

The complex field distribution in the hologram plane $\Psi_{H}(x, y)$, is just the sum of the object and reference waves:

$$
\Psi_{H}(x, y)=a(x, y)+r(x, y)
$$

Therefore, the intensity in the recording plane is

$$
\begin{aligned}
I_{H}(x, y) & =\left|\Psi_{H}(x, y)\right|^{2} \\
& =r_{0}^{2}+a(x, y) a^{*}(x, y)+a(x, y) r^{*}(x, y)+a^{*}(x, y) r(x, y)
\end{aligned}
$$

The recording medium, of course, responds to the exposure which is given by

$$
E=\imath I_{H}=\imath\left[r_{0}^{2}+a a^{*}+a r^{*}+a^{*} r\right],
$$


where $\tau$ is the exposure time. Although the functional dependence of $a$ and $r$ is not explicitly shown in the above expression, it is understood that both quantities are functions of $x$ and $y$. The variation of exposure about the bias point may be obtained by subtracting the bias exposure, $\tau r_{0}{ }^{2}$, from both sides of Equation (2.67), i.e.,

$$
E^{\prime}=E-\tau r_{0}^{2}=\tau\left[a a^{*}+a r^{*}+a^{*} r\right] .
$$

The response of the recording medium is given by its transmittance-exposure curve which, for the purpose of analysis, is approximated by a polynomial, i.e.,

$$
t\left(E^{\prime}\right)=c_{0}+c_{1} E^{\prime}+c_{2} E^{\prime 2}+\cdots+c_{n} E^{\prime n},
$$

where $t$ denotes the transmittance of the processed film, $c_{0}$ is the bias transmittance due to the reference beam alone and $n$ is the number of terms required to give a suitable approximation to the actual curve. Goodman and Knight [1968] have observed that an excellent approximation of the transmittance-exposure curve may be obtained by using $n=3$; however, a two-term polynomial representation will be sufficient to account for the occurrence of the in-line false image. Hence the following quadratic approximation will be used:

$$
t\left(E^{\prime}\right)=c_{0}+c_{1} E^{\prime}+c_{2} E^{\prime 2}
$$


Using Equation (2.68), $c_{2} E^{\prime 2}$ may be expressed in terms of the reference and object waves as follows:

$$
\begin{aligned}
c_{2} E^{\prime 2}=c_{2} c^{2}\left[a^{2} a^{* 2}+a^{2} r^{* 2}+\right. & a^{* 2} r^{2}+2 a^{2} a^{*} r^{*} \\
& \left.+2 a a^{* 2} r+2 a a^{*} r r^{*}\right] .
\end{aligned}
$$

If the hologram is illuminated by the original reference wave $r$, the complex field distribution immediately to the right of the hologram plane is given by the product $r\left(E^{\prime}\right)$. Therefore, the individual terms in the above expression together with the remaining terms in Equation (2.70) will each contribute to the total complex field distribution. The component of the field distribution due to the fourth term in Equation (2.71) is given by

$$
\Psi_{4 H}=r c_{2} \tau^{2}\left[2 a^{2} a^{*} r^{*}\right]=2 c_{2} \tau^{2} r_{0}^{2} a^{2} a^{*}
$$

This quantity is of particular interest since it accounts for the in-line false image.

Substituting the expression for $a(x, y)$ from Equation (2.64a) into the above equation yields

$$
\begin{aligned}
\Psi_{4 H}(x, y) & =2 c_{2} \tau a_{0}^{3} r_{0}^{2} \exp [i k z]\left[1+\frac{i}{\lambda z} \bar{A}(x, y)\right]^{2}\left|1-\frac{i}{\lambda z} \bar{A}^{*}(x, y)\right| \\
& =2 c_{2} \tau a_{0}^{3} r_{0}^{2} \exp [i k z]\left[1-\frac{i}{\lambda z} \bar{A}^{*}(x, y)+\text { other terms } \mid\right.
\end{aligned}
$$

The contribution of $\Psi_{4 H}(x, y)$ in a plane located a distance $z$ to the 
right of the hologram plane may be obtained by applying the HuygensFresnel Principle, i.e.,

$$
\begin{aligned}
\Psi_{4 I}\left(\xi^{\prime}, \eta^{\prime}\right) & =-\frac{i}{\lambda z} \exp [i k z] \\
& \times \iint_{-\infty}^{+\infty} \Psi_{4 H}(x, y) \exp \left\{i \frac{k}{2 z}\left[\left(\xi^{\prime}-x\right)^{2}+\left(\eta^{\prime}-y\right)^{2}\right]\right\} d x d y \quad,(2.74)
\end{aligned}
$$

where $\xi^{\prime}$ and $\eta^{\prime}$ denote the coordinates in this plane. Substituting Equation (2.73) into (2.74) yields, after a straightforward caclulation,

$$
\Psi_{4 I}\left(\xi^{\prime}, \eta^{\prime}\right)=2 c_{2} \tau a_{0}^{3} r_{0}^{2} \exp [i 2 k z]\left[1-A\left(\xi^{\prime}, \eta^{\prime}\right)+\text { other terms }\right]
$$

The above equation indicates that the field distribution in the plane of the original object is reproduced in the $\xi^{\prime} \cdot \eta^{\prime}$ plane, which is located a distance $2 z$ away from the virtual image plane. Hence, the resulting false image occupies the same location as the real image produced by an in-line hologram. It is of interest to note that a false image would not occur at this location if the object was replaced by its complement (i.e., a hole in an otherwise black screen). In this case, the undiffracted component of the object wave would be absent and thus Equation (2.73) reduces to a single term, 


$$
\Psi_{4 H}(x, y)=2 c_{2} \tau a_{0}^{3} r_{0}^{2} \exp [i k z]\left[\frac{i}{(\lambda z)^{3}} \bar{A}^{2} \bar{A}^{*}\right]
$$

which clearly does not yield an image in the $\xi^{\prime}-\eta^{\prime}$ plane. It should be pointed out that Bryngdahl and Lohmann [1968] have shown that false images are dependent on the nature of the object (i.e., a hole vs. a particle), however, their analysis does not explicitly account for the in-1ine false image described above.

To illustrate the results of nonlinear recording, an off-axis hologram of $50 \mu$ particles was recorded onto a Kodak High Resolution Plate Type 1A, using a reference-to-object-beam ratio of approximately $4: 1$. A photograph of a portion of the real image plane is shown in Fig. 2.15a. As expected, dark images on a bright background are observed. A photograph of a portion of the false image plane is shown in Fig. 2.15b; the images are weak but nevertheless distinguishable from the background. Unlike the real images, it is observed that the false images are brighter than the background. The relative intensity of the false images with respect to the background may be explained with the help of the polynomial which describes the transmittance-exposure curve (see Equation $(2.70))$. For a negative transparency, the coefficient, $c_{1}$, of the linear term is clearly a negative quantity, whereas the sign of the quadratic coefficient, $c_{2}$, depends upon the nature of the nonlinearity. If $c_{2}$ is positive, then it may be shown that the 


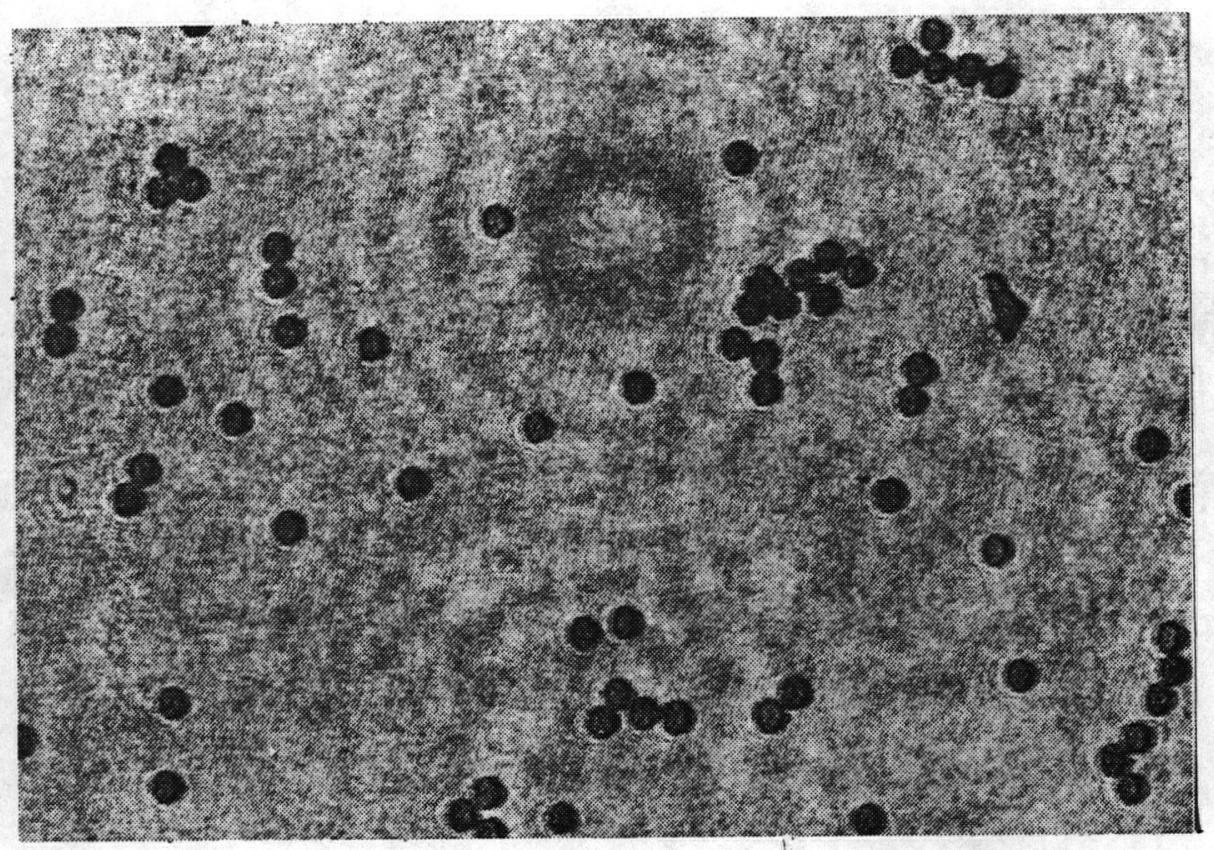

(a)

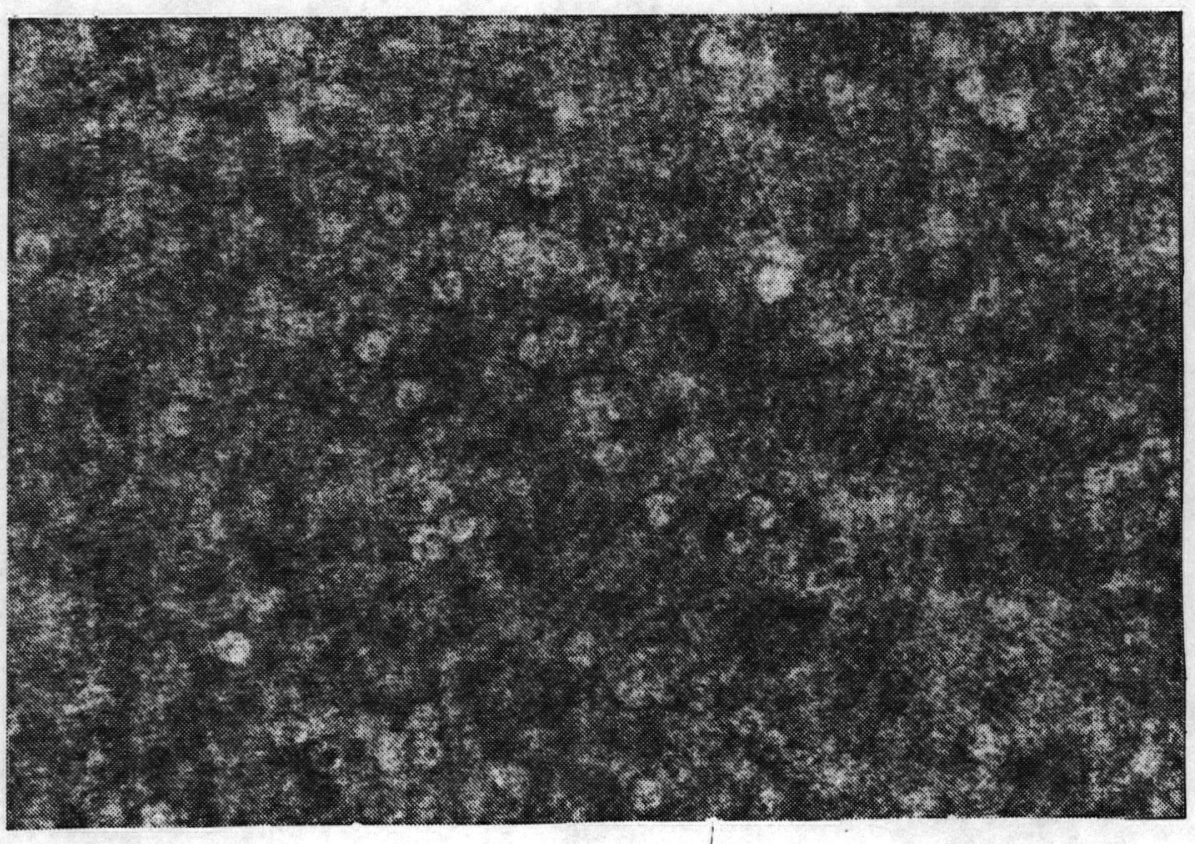

(b)

Fig. 2.15. (a) Real images from an off-axis hologram of $50 \mu$ particles. (b) False images from a nonlinearly recorded off-axis hologram of $50 \mu$ particles. 
intensity of the false images is greater than the background intensity.

As a final example, the optical Fourier transform obtained from an off-axis hologram is shown in Fig. 2.16. In fact, the transform pattern was produced from the same nonlinear hologram used to obtain the reconstructed images shown in Figs. 2.15a and b. It is observed that the zone lens in the transform plane is quite distinct even though the false images in Fig. 2.15b are weak. Hence, if the reason for using off-axis holography is to eliminate the zone lens in the transform plane, the recording process must be 1inear.

\subsection{Summary}

The transform patterns of the reconstructed images from inline and off-axis holograms were examined. It was observed that the transform pattern of an in-line hologram is similar to the total diffraction pattern produced by an actual particle sample. However, due to the on-axis images characteristic of an in-line hologram, additional structure is present in the transform plane of the hologram; i.e., structure dependent on the distribution of particles along the $z$-axis. This structure was shown to be a zone lens if the particles are located in a plane parallel to the hologram plane. If the particles are distributed throughout a volume, then the structure is more complicated since it is dependent on the 


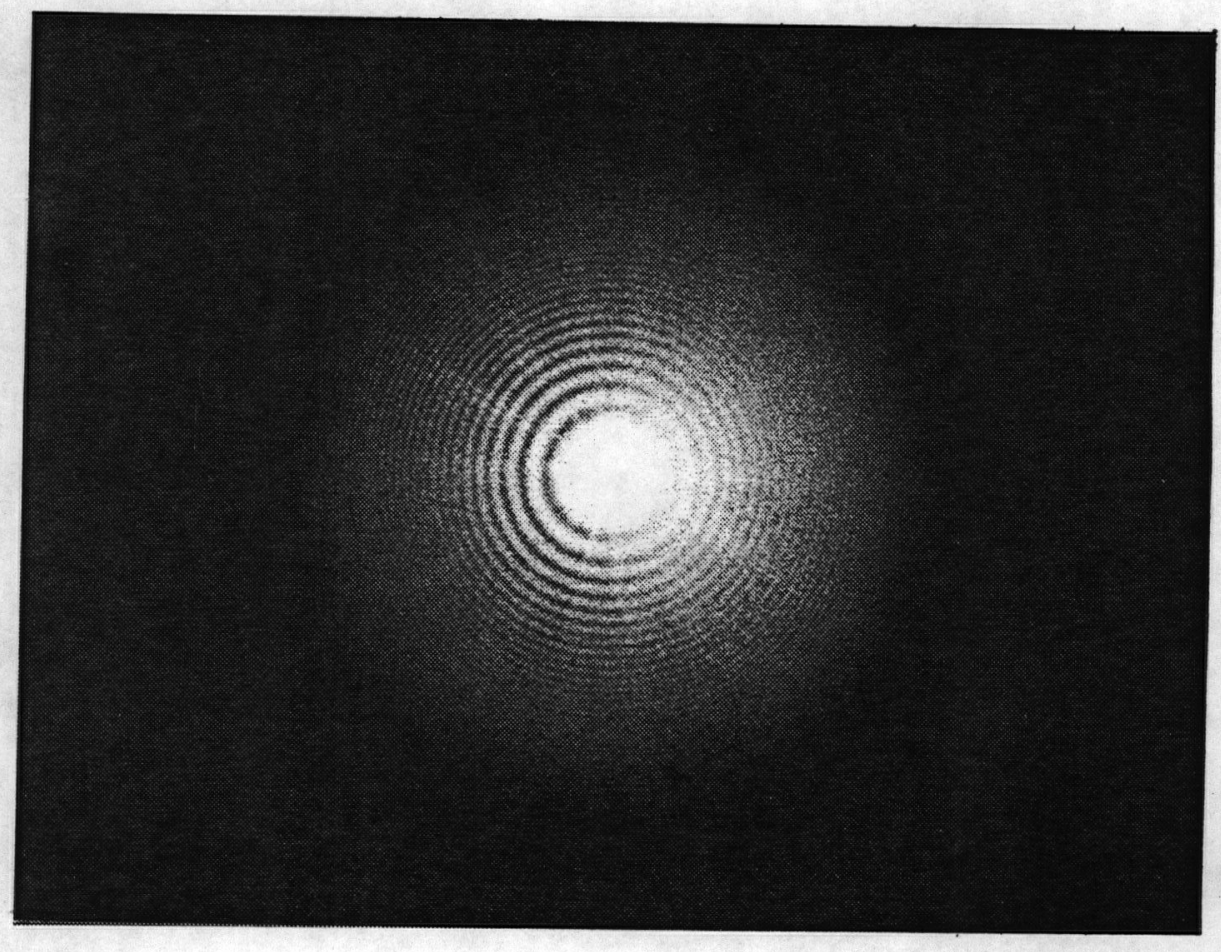

Fig. 2.16. Fourier transform pattern obtained from a nonlinearly recorded off-axis hologram of $50 \mu$ particles. A zone lens is formed in the Fourier plane as a result of the in-line false images. 
probability density function that characterizes the distribution of particles along the $z$-axis.

In situations where the $z$-dependent structure is a problem, off-axis holography may be used. Since the real and virtual images produced by an off-axis hologram are not located on the same axis, the transform pattern produced by an off-axis hologram is, in principle, equivalent to that produced by an actual particle sample. However, as shown in Section 2.3.2., the off-axis hologram must be recorded linearly, otherwise, false images are produced which cause the intensity in the transform plane to resemble the transform pattern produced by an in-line hologram. 


\section{Chapter III}

\section{Doubly Exposed Holograms: Particle Displacement \\ Measurement in the Fourier Plane}

\subsection{Introduction}

As discussed in Chapter 1, a double-exposure hologram of a particle field contains displacement (and, hence, velocity) information for all particles within a given sample volume. In practice, of course, it may be difficult to obtain the velocity information directly from the holographic reconstruction of the sample volume. However, it was noted that the velocity information is displayed in a form suitable for automatic analysis in the transform plane of the reconstructed particle images.

In this chapter, the theoretical aspects of doubly exposed holograms (in-line and off-axis) and the corresponding Fourier transforms are first examined. The effects of 1- and 2- dimensional velocity distributions are then addressed and a procedure for measuring the velocity distribution from the intensity pattern in the transform plane is developed.

3.2 The Fourier transform of a doubly exposed in-line far-field hologram

In chapter 2, the transform patterns produced by singleexposure holograms were studied and the results obtained will now be 
generalized to describe the double-exposure case. The field distribution in the Fourier transform plane of a single-exposure far-field hologram is given in Equation (2.31) and is rewritten below in a slightly different form:

$$
\Psi_{F}(u, v)=i \frac{2}{\lambda f} \cos ^{2}\left(\frac{\pi z \rho^{2}}{\lambda f^{2}}\right) \sum_{n=1}^{N} F\left\{A\left(\xi-\xi_{n}, \eta-\eta_{n}\right)\right\}
$$

where $\xi_{n}$ and $\eta_{n}$ denote the coordinates of the $n^{\text {th }}$ particle in the object plane and $F\{\cdot\}$ denotes a Fourier transform. In comparing Equation (2.31) to (3.1), it is observed that the only difference between the two expressions is the manner in which the Fourier transform of the $n^{\text {th }}$ particle is designated. In Equation (3.1), the particle coordinates appear explicitly (unlike-Equation (2.31)) and this representation will be useful when considering the transform pattern resulting from a double-exposure hologram. Furthermore, it should be remembered that the above expression is valid away from the origin; near the origin other contributions will be present (see Section 2.21).

When forming a double-exposure hologram, each of the particles in the sample will undergo a displacement between exposures. The displacement of the $n^{\text {th }}$ particle along the $\xi$-axis is denoted by $\Delta \xi_{n}$ and, similarly, the displacement along the $\eta$-axis is denoted by $\Delta \eta_{n}$. As a result of the displacement, each particle will be recorded twice, i.e., once for each exposure. The hologram thus formed is effectively a recording of $2 N$ particles (where the sample volume is 
assumed to contain $N$ particles). Hence, the field in the transform plane of a doubly exposed in-line hologram may be obtained from Equation ( 3.1 ) by including the additional contributions which result from the second exposure, namely,

$$
\begin{aligned}
\Psi_{F}(u, v)=i \frac{2}{\lambda f} \cos ^{2}\left(\frac{\pi z p^{2}}{\lambda f^{2}}\right)\left[\sum_{n=1}^{N} F\left\{A\left(\xi-\xi_{n}, \eta-\eta_{n}\right)\right\}\right. \\
\left.+\sum_{n=1}^{N} F\left\{A\left(\xi-\xi_{n}-\Delta \xi_{n}, \eta-\eta_{n}-\Delta \eta_{n}\right)\right\}\right]
\end{aligned}
$$

As a simplification, it is assumed that the motion of the individual particles between exposures is purely translational for nonspherical particles and that all particles are identical in size and shape. Applying the shift theorem of Fourier analysis (see Equation $(2.32))$ to evaluate the transforms in Equation (3.2) and then taking the squared modulus yields an expression for the the intensity in the transform plane:

$$
I_{F}(u, v)=\left|\bar{A}\left(\frac{u}{\lambda f}, \frac{v}{\lambda f}\right)\right|^{2} \cos ^{2}\left(\frac{\pi z \rho^{2}}{\lambda f^{2}}\right)\left[D_{1}+D_{2}+D_{3}+D_{4} \mid\right.
$$

where an unessential constant factor has been omitted and

$$
\begin{aligned}
& D_{1}=\sum_{n=1}^{N} \sum_{m=1}^{N} \exp \left\{-i \frac{2 \pi}{\lambda f}\left[u\left(\xi_{n}-\xi_{m}\right)+v\left(\eta_{n}-\eta_{m}\right)\right]\right\} \\
& D_{2}=\sum_{n=1}^{N} \sum_{m=1}^{N} \exp \left\{-i \frac{2 \pi}{\lambda f}\left[u\left(\xi_{n}+\Delta \xi_{n}-\xi_{m}-\Delta \xi_{m}\right)+v\left(\eta_{n}+\Delta \eta_{n}-\eta_{m}-\Delta \eta_{m}\right)\right]\right\}
\end{aligned}
$$




$$
\begin{aligned}
& D_{3}=\sum_{n=1}^{N} \sum_{m=1}^{N} \exp \left\{-i \frac{2 \pi}{\lambda f}\left[u\left(\xi_{n}-\xi_{m}-\Delta \xi_{m}\right)+v\left(\eta_{n}-\eta_{m}-\Delta \eta_{m}\right)\right]\right\}, \\
& D_{4}=\sum_{n=1}^{N} \sum_{m=1}^{N} \exp \left\{-i \frac{2 \pi}{\lambda f}\left[u\left(\xi_{n}+\Delta \xi_{n}-\xi_{m}\right)+v\left(\eta_{n}+\Delta \eta_{n}-\eta_{m}\right)\right]\right\}
\end{aligned}
$$

The intensity $I_{F}(u, v)$ is, of course, a random variable since the coordinates of the $n^{\text {th }}$ particle, $\xi_{n}$ and $\eta_{n}$, are random in general. Hence, the expected value of the intensity distribution is of interest, i.e.,

$$
\begin{aligned}
&\left\langle I_{F}(u, v)>=\left|\tilde{\mathrm{A}}\left(\frac{u}{\lambda f}, \frac{v}{\lambda f}\right)\right|^{2} \cos ^{2}\left(\frac{\pi z \rho^{2}}{\lambda f^{2}}\right)\right. \\
&\left.\times\left|\left\langle D_{1}\right\rangle+<D_{2}\right\rangle+\left\langle D_{3}\right\rangle+<D_{4}\right\rangle \mid .
\end{aligned}
$$

The expected values of $D_{1}, D_{2}, D_{3}$ and $D_{4}$ may be evaluated by following the procedure given in Section 2.2.2 in which the expected value of the intensity distribution in the transform plane of a single-exposure hologram was obtained. Thus,

$$
\begin{aligned}
& <D_{1}>=N \\
& <D_{2}>=N \\
& <D_{3}>=\sum_{n=1}^{N} \exp \left\{+i \frac{2 \pi}{\lambda f}\left(u \Delta \xi_{n}+v \Delta \eta_{n}\right)\right\},
\end{aligned}
$$




$$
<D_{4}>=\sum_{n=1}^{N} \exp \left\{-i \frac{2 \pi}{\lambda f}\left(u \Delta \xi_{n}+v \Delta \eta_{n}\right)\right\},
$$

and

$$
\begin{aligned}
&<I_{F}(u, v)>=\left|\tilde{A}\left(\frac{u}{\lambda f}, \frac{v}{\lambda f}\right)\right|^{2} \cos ^{2}\left(\frac{\pi z \rho^{2}}{\lambda f^{2}}\right) \\
& \times\left\{2 N+2 \sum_{n=1}^{N} \cos \left[\frac{2 \pi}{\lambda f}\left(u \Delta \xi_{n}+v \Delta \eta_{n}\right) \mid\right\}\right.
\end{aligned}
$$

The above equation is the central result governing the displacement measurement problem. As indicated by the summation term in Equation (3.6), each particle pair related by translation contributes a set of cosinusoidal fringes of a particular frequency to the total diffraction pattern. The fringe frequency and orientation are directly related to the magnitude and direction, respectively, of the displacement of a given particle. In addition to the term describing the cosinusoidal fringes, the expression for the intensity in the transform plane (Equation (3.6)) contains two other terms, $|\bar{A}(\cdot)|^{2}$ and $\cos ^{2}(\cdot)$. It should be recalled from the analysis describing the transform pattern produced by a single-exposure farfield hologram (Section 2.2.2) that $\bar{A}(\cdot)$ denotes the Fourier transform of of a typical particle's cross-sectional shape and $\cos ^{2}(\cdot)$ describes a zone lens. (While a zone lens is usually described by $a+b \cos \left(c p^{2}\right)$ where $a, b$ and $c$ are constants, it should 
be recalled that $2 \cos ^{2} \theta=1+\cos 2 \theta$ and thus the second term in Equation (3.6) does indeed describe a zone lens.) Hence, the intensity pattern in the transform plane of a double-exposure hologram is similar to that produced by a single-exposure hologram; the difference, of course, is that the set of cosinusoidal fringes only appears in the double-exposure case.

Typically, the particle displacements in Equation (3.6) are random and may be characterized in terms of a probability density function. Since our objective is to determine the distribution of displacements based on measurements of the intensity pattern in the transform plane, it is necessary to establish the relationship between the density function and Equation (3.6). First, it should be noted that the sample mean of $N$ random variables, $c_{1}, c_{2}, \ldots, c_{N}$, is defined as the arithmetic average (Davenport and Root [1958]), i.e.,

$$
\bar{m}=\frac{1}{N} \sum_{n=1}^{N} c_{n}
$$

For the problem at hand,

$$
c_{n}=\cos \left[\frac{2 \pi}{\lambda f}\left(u \Delta \xi_{n}+v \Delta \eta_{n}\right)\right] .
$$

For large $N$, it may be shown that the sample mean converges in probability to the actual mean (Davenport and Root [1958]), i.e., 


$$
\bar{m}=\iint_{-\infty}^{+\infty} p\left(s_{1}, s_{2}\right) \cos \left[\frac{2 \pi}{\lambda f}\left(u s_{1}+v s_{2}\right)\right] d s_{1} d s_{2}
$$

where $p\left(s_{1}, s_{2}\right)$ is the density function describing the particle displacements. Assuming that $N$ is sufficiently large, Equations $(3.7)-(3.9)$ can be combined to yield

$$
\sum_{n=1}^{N} \cos \left[\frac{2 \pi}{\lambda f}\left(u \Delta \xi_{n}+v \Delta \eta_{n}\right)\right]=N \iint_{-\infty}^{+\infty} p\left(s_{1}, s_{2}\right) \cos \left[\frac{2 \pi}{\lambda f}\left(u s_{1}+v s_{2}\right)\right] d s_{1} d s_{2} \cdot(3.10)
$$

Combining Equation (3.6) with the above equation gives the desired expression for the intensity in the transform plane:

$$
<I_{F}(u, v)>=I_{0}(u, v)\left\{1+\iint_{-\infty}^{+\infty} p\left(s_{1}, s_{2}\right) \cos \left[\frac{2 \pi}{\lambda f}\left(u s_{1}+v s_{2}\right)\right] d s_{1} d s_{2}\right\},
$$

where

$$
I_{0}(u, v)=2 N\left|\tilde{A}\left(\frac{u}{\lambda f}, \frac{v}{\lambda f}\right)\right|^{2} \cos ^{2}\left(\frac{\pi z \rho^{2}}{\lambda f^{2}}\right) .
$$

If the particle motion is confined to one direction, then a onedimensional density function, $p\left(s_{1}\right)$, will be sufficient to describe the distribution of displacements. Thus, the intensity in the transform plane may be written as

$$
<I_{F}(u, v)>=I_{0}(u, v)\left\{1+\int_{-\infty}^{+\infty} p\left(s_{1}\right) \cos \left(\frac{2 \pi u s_{1}}{\lambda f}\right) d s_{1}\right\} .
$$


3.3 Illustrative examples: Some specific velocity distributions and the corresponding Fourier transform patterns

In the previous section, a rather general expression for the intensity in the transform plane of a double-exposure far-field hologram was obtained (Equation 3.11)). However, to illustrate how a distribution of particle displacements affects the transform pattern, it is perhaps helpful to consider several specific distributions.

\subsubsection{All particles have the same displacement between expsoures}

It is first assumed that all particles undergo the same displacement between expsoures. The magnitude of the displacement is denoted by $\Delta$ and all particles are assumed to move parallel to the horizontal axis. Thus, the density function is given by

$$
p\left(s_{1}\right)=\delta\left(s_{1}-\Delta\right)
$$

The intensity in the transform plane of the resulting far-field hologram may then be obtained by substituting the above expression into Equation (3.13), yielding

$$
<I_{F}(u, v)>=I_{0}(u, v)\left[1+\cos \left(\frac{2 \pi u \Delta}{\lambda f}\right) \mid .\right.
$$

Thus, the displacement information is represented by the cosinusoidal term in the above expression; specifically, the frequency of the cosine is directly proportional to the particle 
displacement. This particular example may readily be demonstrated experimentally by forming a double exposure hologram of particles suspended on a glass plate. The plate is then translated between exposures, thereby displacing all particles by an equal amount. The optical Fourier transforms of two far-field holograms produced in this manner are shown in Figs. 3.1a and b. (A photograph of reconstructed images obtained from a doubly exposed far-field hologram is shown in Fig 3.1c.) All the features described by Equations (3.15) and (3.12) are observed in the photographs, i.e., the overall envelope $|\tilde{A}(\cdot)|^{2}$, the zone lens, and the set of cosinusoidal fringes. It may appear that the theoretical description is incomplete since multiple zone lenses are present in Fig $3.1 \mathrm{~b}$, however, the additional zone lenses are a result of the Moiré effect. This may be verified by using standard trigonometric identities to rewrite the expression describing the intensity in the transform plane (Equation (3.15) as

$$
\begin{aligned}
<I_{F}(u, v)>=N\left|\overline{\mathrm{A}}\left(\frac{u}{\lambda f}, \frac{v}{\lambda f}\right)\right|^{2}\left\{1+\cos \frac{2 \pi u \Delta}{\lambda f}+\cos \frac{2 \pi z \rho^{2}}{\lambda f^{2}}\right. \\
-\frac{1}{2} \cos \left[\frac{2 \pi z}{\lambda f^{2}}\left[\left(u+\frac{f \Delta}{2 z}\right)^{2}+v^{2} \mid-\left(\frac{f \Delta}{2 z}\right)^{2}\right]\right. \\
\left.+\frac{1}{2} \cos \left[\frac{2 \pi z}{\lambda f^{2}}\left[\left(u-\frac{f \Delta}{2 z}\right)^{2}+v^{2}\right]-\left(\frac{f \Delta}{2 z}\right)^{2}\right]\right\},
\end{aligned}
$$

where $u$ and $v$ are the coordinates in the transform plane and 


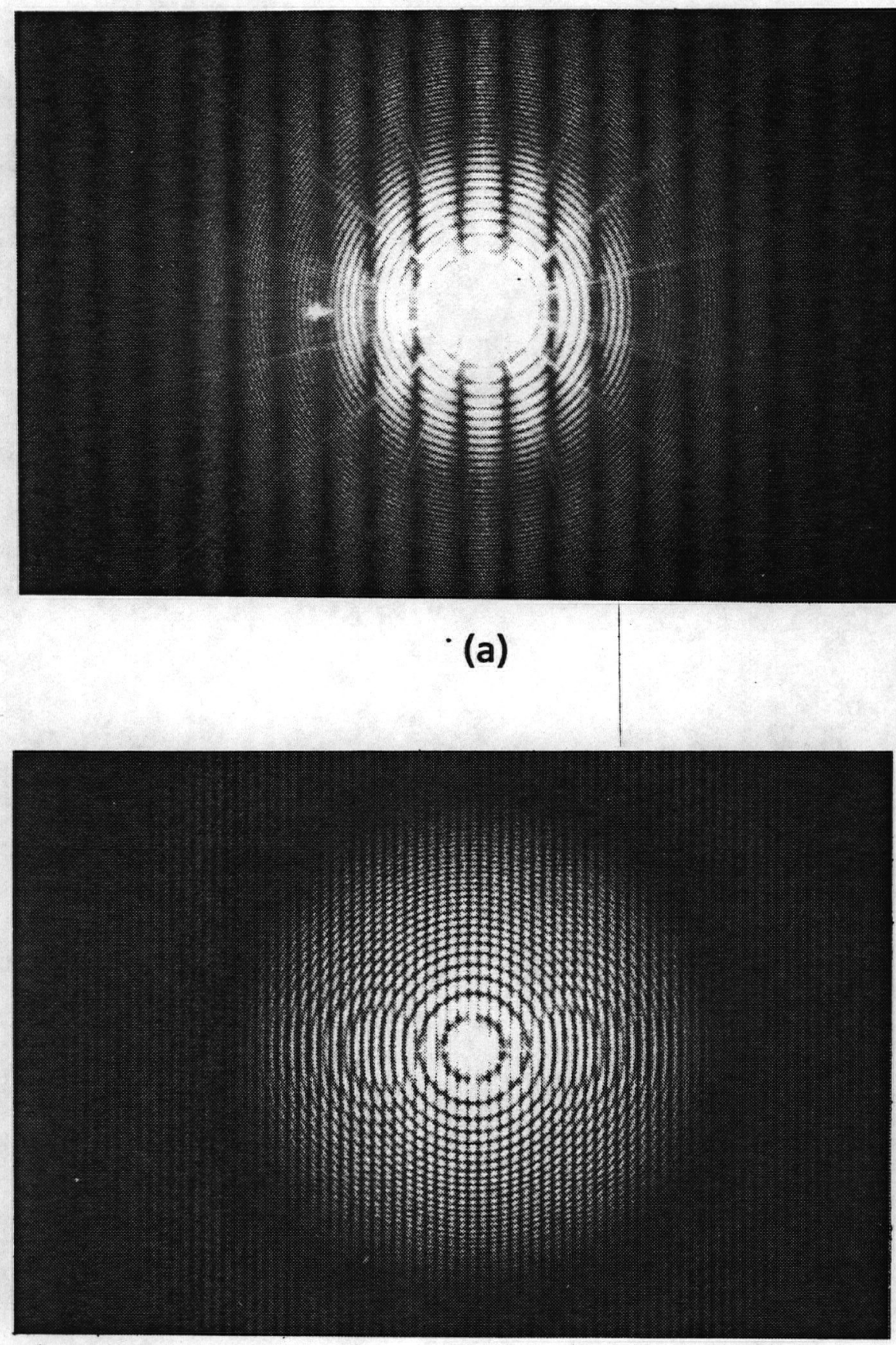

(b)

Fig. 3.1. (a) Optical Fourier transform pattern produced by a double-exposure far-field hologram of $80 \mu$ particles. The displacement between exposures was $0.3 \mathrm{~mm}$. (b) Same as (a) except $50 \mu$ particles were used and the displacement was $1 \mathrm{~mm}$. 


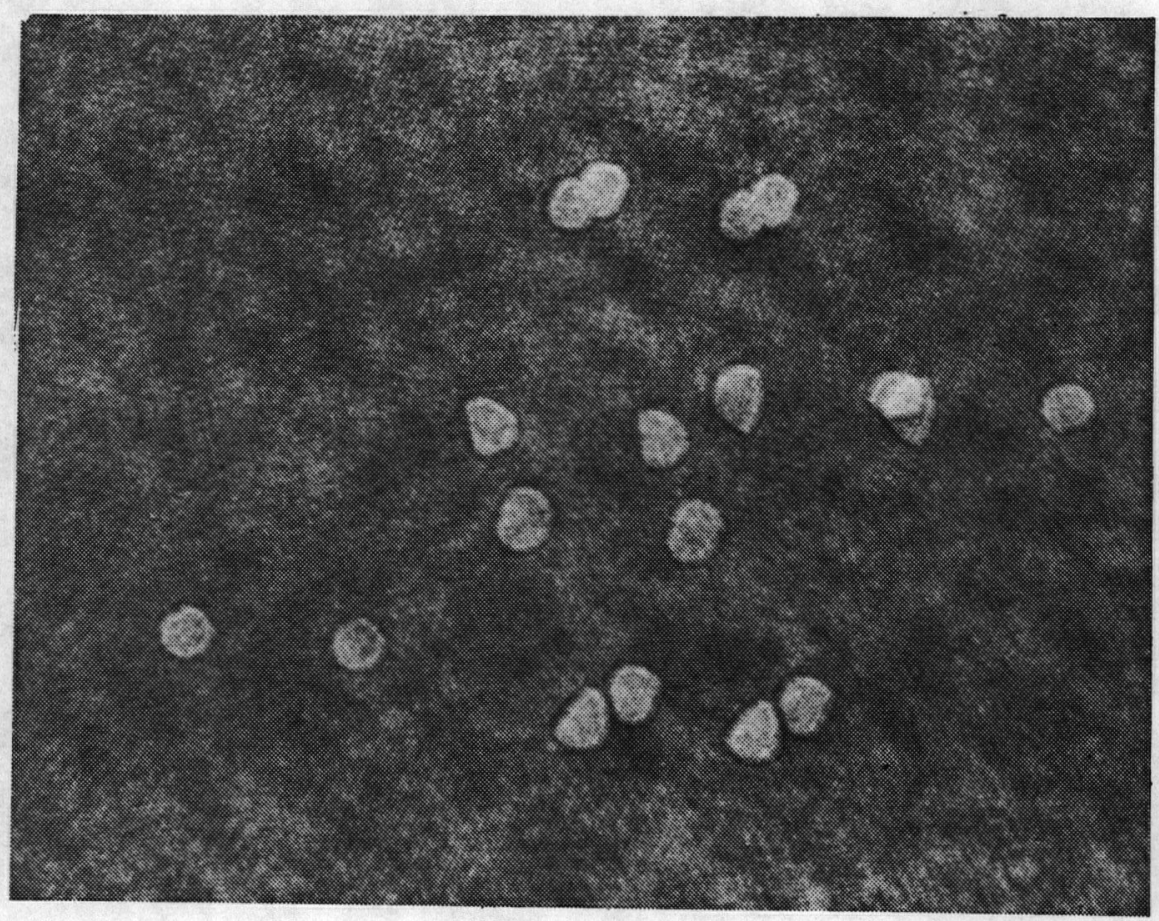

Fig. 3.1. (c) A photograph of reconstructed images obtained from a double-exposure far-field hologram of $80 \mu$ particles. 
$\rho^{2}=u^{2}+v^{2}$. The above equation indicates that three zone lens patterns will be present: one is centered at $(u=0, v=0)$, another at $(u=-f \Delta / 2 z, v=0)$ and the third is located at $(u=+f \Delta / 2 z, v=0)$. There is still some discrepancy between theory and experiment since five zone lens patterns are observed in Fig. 3.1b while Equation (3.15) accounts for only three of these. However, the additional zone lens patterns result from nonlinearities introduced when photographing the transform pattern.

\subsubsection{The effect of a uniform distribution of particle displacements}

In this example, the direction of particle motion is assumed to be parallel to the horizontal axis and the magnitudes of the displacements are assumed to be uniformly distributed over the interval, $b<s_{1}<c$. Thus, the density function is given by

$$
p\left(s_{1}\right)=\frac{1}{b-c} \quad \text { for } \quad b<s_{1}<c
$$

Substituting the above expression into Equation (3.13) yields an expression for the intensity in the transform plane,

$$
<I_{F}(u, v)>=I_{0}(u, v)\left[1+\operatorname{sinc}\left(\frac{2 u}{\lambda f} \frac{(c-b)}{2}\right) \cos \left(\frac{2 \pi u}{\lambda f} \frac{(c+b)}{2}\right)\right],
$$

where $\operatorname{sinc}(x)=\sin (\pi x) /(\pi x)$. Thus, a cosinusoidal term is once again present, however, the frequency is determined by the mean particle displacement. In addition, the cosine is modulated by a sinc function whose width is determined by the width of the density 
function. That is, the width of the density function determines the visibility of the fringes in the transform plane. If the particle displacements are known to be uniformly distributed over some interval, then that interval may be determined directly from measurements of the frequency and visibility of the fringes in transform plane of the doubly exposed hologram.

\subsubsection{The effect of a Gaussian distribution of particle displacements}

The direction of particle motion is again assumed to be parallel to the horizontal axis but the displacements are assumed to be characterized by a Gaussian probability density function, i.e.,

$$
p\left(s_{1}\right)=\frac{1}{\sqrt{2 \pi} \sigma} \exp \left[\frac{\left(s_{1}-\mu\right)^{2}}{2 \sigma^{2}}\right],
$$

where $\mu$ and $\sigma$ denote the mean and standard deviation, respectively. Substituting this density function into Equation (3.13) yields the intensity in the transform plane,

$$
<I_{F}(u, v)>=I_{0}(u, v)\left\{1+\exp \left[-\frac{2 \pi^{2} \sigma^{2} u^{2}}{\lambda^{2} f^{2}}\right] \cos \left(\frac{2 \pi \mu u}{\lambda f}\right)\right\} .
$$

The above result is similar to that obtained for the case of a uniform distribution in that the frequency of the cosine is determined by the mean particle displacement and the visibility of the fringes is determined by the width of the density function. 


\subsubsection{The effect of a Gaussian distribution of displacements in two dimensions}

Each of the previous examples assumes that all particles under investigation travel in a given direction between exposures. However, it is also of interest to consider the case in which the particles are allowed to move in any direction within a plane parallel to the hologram plane; that is, a two-dimensional distribution of displacements. In particular, it will be assumed that the density function describing the particle displacements is given by

$$
p\left(s_{1}, s_{2}\right)=\frac{1}{2 \pi \sigma_{1} \sigma_{2}} \exp \left\{-\frac{1}{2}\left[\frac{\left(s_{1}-\mu_{1}\right)^{2}}{\sigma_{1}^{2}}+\frac{\left(s_{2}-\mu_{2}\right)^{2}}{\sigma_{2}^{2}}\right]\right\},
$$

i.e., the random variables describing the particle displacements in the object plane are jointly normal and independent. The quantities $\mu_{1}$ and $\sigma_{1}$ denote the mean and standard deviation of displacement along the horizontal $(\xi-)$ axis in the object plane and similarly, $\mu_{2}$ and $\sigma_{2}$ denote the mean and standard deviation of displacement along the vertical $\left(\eta^{-}\right)$axis. In practice, this density function (with $\mu_{2}=0$ ) might be used to describe a conical-type flow.

Substituting the above density function into Equation (3.11) yields the intensity in the transform plane of the far-field hologram, 


$$
<I_{F}(u, v)>=I_{0}(u, v)\left\{1+\exp \left[-\frac{2 \pi^{2}}{\lambda^{2} f^{2}}\left(\sigma_{1}^{2} u^{2}+\sigma_{2}^{2} v^{2}\right)\right] \cos \left[\frac{2 \pi}{\lambda f}\left(\mu_{1} u+\mu_{2} v\right)\right]\right\} .
$$

Once again a cosinusoidal term carrying the velocity information is present in the transform plane. In this case, the frequency and orientation of the cosine depend on both the mean horizontal displacement, $\mu_{1}$, and the mean vertical displacement, $\mu_{2}$. The visibility of the fringes, which is determined by the exponential term, depends on both $\sigma_{1}$ and $\sigma_{2}$. Since the exponential term is a function of the coordinates $u$ and $v$, the spatial extent of the fringe pattern is limited to a region centered about the origin of the transform plane. In contrast, a one-dimensional distribution of displacements limits the extent of the fringe pattern in one direction only $(c f$. Equation $(3.20))$.

3.3.5 The relationship between the width of the density function and the ability to obtain it from intensity data measured in the transform plane of a doubleexposure hologram

From the examples presented, it is observed that there is an inverse relationship between the width of the density function which describes the distribution of particle displacements and the visibility of the fringes in the transform plane of the hologram. Specifically, for a given mean displacement, fewer and fewer fringes remain visible as the width of the density function increases, implying that there is an upper limit to the distribution width which may be measured with this technique. This inverse 
relationship may be understood by examining the general expression that describes the intensity in the transform plane of a doubleexposure far-field hologram (i.e., Equation (3.13)) for onedimensional distributions or Equation (3.11) for two-dimensional distributions). For example, in the case of a one-dimensional distribution, the displacement information in the transform plane is described by the term in braces in Equation (3.13) which is rewritten below:

$$
\left\{1+\int_{-\infty}^{+\infty} p\left(s_{1}\right) \cos \left(\frac{2 \pi u s_{1}}{\lambda f}\right) d s_{1}\right\} .
$$

Clearly, the above integral is the real part of the Fourier transform of $p\left(s_{1}\right)$ (assuming that $p\left(s_{1}\right)$ is real) and it is known from Fourier transform theory that the width of the function being transformed is inversely related to the width of the transform of that function (see, for example, Born and Wolf [1975]). Hence, if the density function is excessively wide, it cannot be determined from intensity data measured in the transform plane of a doubly exposed hologram.

The above discussion presented a rather formal explanation regarding the maximum allowable distribution width which may be measured; however, the reason for this upper limit may be understood on an intuitive basis. For example, if all particles in the sample undergo the same displacement between exposures, there is obviously a high degree of correlation between the hologram recorded during 
the first exposure and the hologram recorded during the second exposure. Thus, the optical Fourier transform of the resulting double-exposure far-field hologram contains fringes of high contrast. When the individual particles undergo slightly different displacements between exposures, the degree of correlation between the successive holograms (which together comprise the doubleexposure hologram) is reduced, yielding a transform pattern which exhibits fringes having relatively lower contrast. As the particle displacements become increasingly random (i.e., the density function describing the displacements becomes wider), the amount of correlation between the successive holograms becomes negligible. Hence, fringes are no longer visible in the transform plane of the doubly exposed hologram and displacement measurement is not possible.

3.4 The use of double-exposure off-axis holography for particle displacement and velocity measurement

Double-exposure off-axis holography can, of course, be used to measure particle displacements. A number of off-axis recording geometries are possible, however, the geometry of interest is the one described in Chapter 2 in connection with single-exposure particle field holography (see Fig. 2.13a). The experimental arrangement for obtaining the optical Fourier transform is shown in Fig. 2.13b.

As shown in the previous chapter (Section 2.3.1), the fundamental difference between the Fourier transform produced by a 
single-exposure off-axis hologram when compared to that produced by a single-exposure in-line hologram is the zone lens term. It should be recalled that the zone lens does not appear in transform pattern produced by a linearly recorded off-axis hologram. Thus, the field in the transform plane of a single-exposure off-axis hologram is given by

$$
\Psi_{F}(u, v)=\sum_{n=1}^{N} F\left\{A\left(\xi-\xi_{n}, \eta-\eta_{n}\right)\right\}
$$

which, apart from the zone lens term, is the same as the expression that describes the field in the transform plane of a single-exposure in-line hologram (see Equation (3.1)). As usual, $\xi_{n}$ and $\eta_{n}$ denote the coordinates of the $n^{\text {th }}$ particle, $F\{\cdot\}$ denotes a Fourier transform and $A(\cdot)$ is the object function which describes the cross-sectional shape of a typical particle.

The field in the transform plane of a doubly exposed off-axis hologram may be obtained from Equation (3.23) by including the additional contributions which results from the second exposure, i.e.,

$$
\Psi_{F}(u, v)=\sum_{n=1}^{N} F\left\{A\left(\xi-\xi_{n}, \eta-\eta_{n}\right)\right\}+\sum_{n=1}^{N} F\left\{A\left(\xi-\xi_{n}-\Delta \xi_{n}, \eta-\eta_{n}-\Delta \eta_{n}\right)\right\}
$$

It should be noted that the above expression was obtained in a manner completely analogous to the analysis leading to Equation (3.2); the difference between Equation (3.2) and (3.24) is, of 
course, the zone lens term. Hence, the analytical results given in the previous sections to describe the intensity in the transform plane of a double-exposure far-field hologram will apply to the offaxis case if the zone lens term is omitted. For example, if the particle displacements are characterized by a one-dimensional density function $p\left(s_{1}\right)$, the intensity in the transform plane of a double-exposure far-field hologram is given by Equation (3.13); the corresponding result for the off-axis case is

$$
\left\langle I_{F}(u, v)\right\rangle=2 N\left|\tilde{\mathrm{A}}\left(\frac{u}{\lambda f}, \frac{v}{\lambda f}\right)\right|^{2}\left\{1+\int_{-\infty}^{+\infty} p\left(s_{1}\right) \cos \left(\frac{2 \pi u s_{1}}{\lambda f}\right) d s_{1}\right\} .
$$

To compare theory with experiment, a double-exposure off-axis hologram was recorded and the resulting optical Fourier transform is shown in Fig. 3.2. $50 \mu$ particles were used in forming the hologram and all particles had the same displacement between exposures. As expected, there is no zone lens present but the transform pattern is otherwise similar to that produced by a doubly exposed in-line farfield hologram (see Figs. 3.1a and 3.2b).

Since the transform patterns produced by double-exposure inline and off-axis holograms are so similar, it is unnecessary to continually develop results for both cases independently. The inline far-field hologram will be considered exclusively in all remaining calculations with the understanding that the corresponding result for an off-axis hologram may be obtained by omitting the zone lens term. 


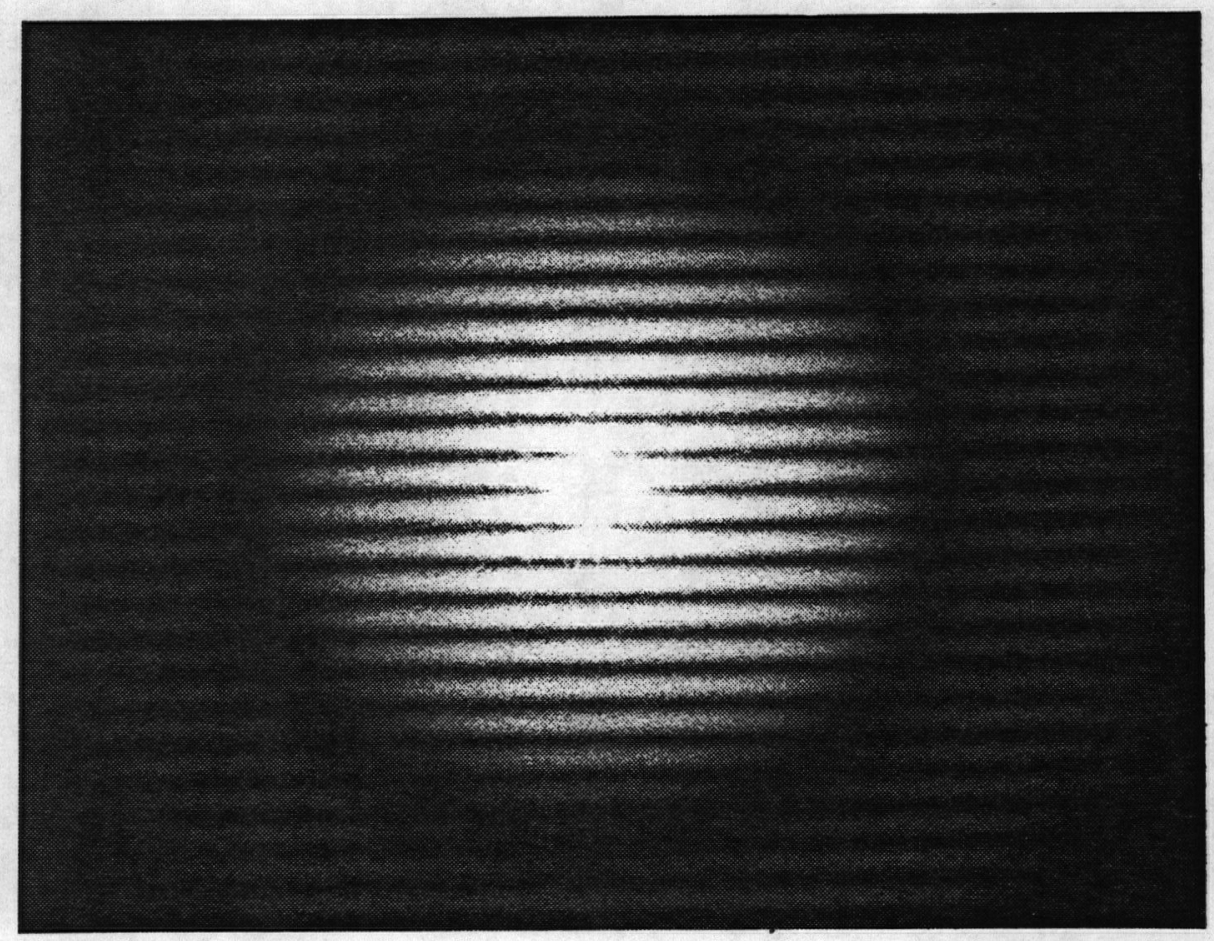

Fig. 3.2. Optical Fourier transform obtained from a double-exposure off-axis hologram of $50 \mu$ particles. 
3.5 A method for obtaining one-dimensional displacement and velocity distributions from intensity measurements in the transform plane of a double-exposure hologram

The examples presented in Sections $3.2 .2-3.2 .4$ indicate that the mean and standard deviation of displacement may be obtained by measuring the spacing and visibility, respectively, of the fringes produced in the transform plane of a double-exposure far-field hologram. This result was obtained by assuming a particular density function and then evaluating the expression for the intensity in the transform plane. However, the form of the density function is usually not known a priori. Hence, it would be desirable to develop a more general approach, one that allows the distribution of displacements to be measured directly from the intensity data in the transform plane of a double-exposure hologram. Such a method is presented in this section.

An expression for the intensity in the transform plane of a double-exposure far-field hologram was derived in Section 3.2 and is given by

$$
<I_{F}(u, v)>=I_{0}(u, v)\left\{1+\int_{-\infty}^{+\infty} p\left(s_{1}\right) \cos \left(\frac{2 \pi u s_{1}}{\lambda f}\right) d s_{1}\right\},
$$

where it is assumed that the particle displacements can be characterized by a one-dimensional density function $p\left(s_{1}\right)$. Clearly, $p\left(s_{1}\right)$ is the desired quantity; the goal is to develop a means 
of obtaining it from the measured intensity data. The first step in obtaining $p\left(s_{1}\right)$ is to divide both sides of the above equation by $I_{0}(u, v)$ yielding

$$
I^{\prime}(u)=\frac{<I_{F}(u, v)>}{I_{0}(u, v)}=1+\int_{-\infty}^{+\infty} p\left(s_{1}\right) \cos \left(\frac{2 \pi u s_{1}}{\lambda f}\right) d s_{1}
$$

For the present discussion it will be assumed that $I_{0}(u, v)$ is known. It should be recalled that $I_{0}(u, v)$ contains a zone lens term (see Equation (3.12)) which, of course, exhibits numerous zeros in the $u, v$-plane. Thus, there appears to be a problem in dividing Equation (3.27) by $I_{0}(u, v)$ due to the zeros of the zone lens. In practice, however, there is no difficulty since the zone lens may effectively be removed from the transform pattern by increasing its frequency (by increasing the object-to-hologram separation) beyond the resolution limit of the detector used to measure $\left\langle I_{F}(u, v)\right\rangle$. Alternatively, off-axis holography may be used to eliminate the zone lens entirely.

The density function may be obtained from Equation (3.27) by taking the Fourier transform of $I^{\prime}(u)$, i.e.,

$$
F\left\{I^{\prime}(u)\right\}=\int_{-\infty}^{+\infty} I^{\prime}(u) \exp [-i 2 \pi u a] d u
$$

where $a$ is the transform variable. Substituting the right-hand side of Equation (3.27) into the above expression yields 


$$
F\left\{I^{\prime}(u)\right\}=\delta(a)+\frac{\lambda f}{2} \int_{-\infty}^{+\infty} p\left(s_{1}\right) \delta\left(s_{1}+\lambda f a\right) d s_{1}+\frac{\lambda f}{2} \int_{-\infty}^{+\infty} p\left(s_{1}\right) \delta\left(s_{1}+\lambda f a\right) d s_{1}
$$

where the $\delta$-function identity,

$$
\delta(a)=\int_{-\infty}^{+\infty} \exp [ \pm i 2 \pi \beta a] d \beta
$$

was used in evaluating the integrations with respect to $u$. Performing the integrations in Equation (3.29) yields

$$
F\left\{I^{\prime}(u)\right\}=\bar{F}(a)=\delta(a)+\frac{\lambda f}{2} p(-\lambda f a)+\frac{\lambda f}{2} p(\lambda f a)
$$

It will be helpful to discuss the above equation in terms of the graphical representation shown in Fig. 3.3 in which $F\left\{I^{\prime}(u)\right\}$ is plotted as a function of the transform variable, $a$. A bell-shaped curve is used to represent the density function $p(\cdot)$. It is observed that $p(\cdot)$ appears at two different locations in the plot; physically, this results from the ambiguity in determining the absolute direction of particle motion from a double-exposure hologram. The separation between the peak value of $p(-\lambda f a)$ and $p(\lambda f a)$ clearly depends on the mean particle displacement. It is therefore desirable to have the mean displacement large enough to assure that $p(-\lambda f a)$ and $p(\lambda f a)$ are well-separated from one another to prevent aliasing. Thus, the density function can, in principle, be obtained by measuring the intensity in the transform plane of a double-exposure hologram and processing the intensity data as 


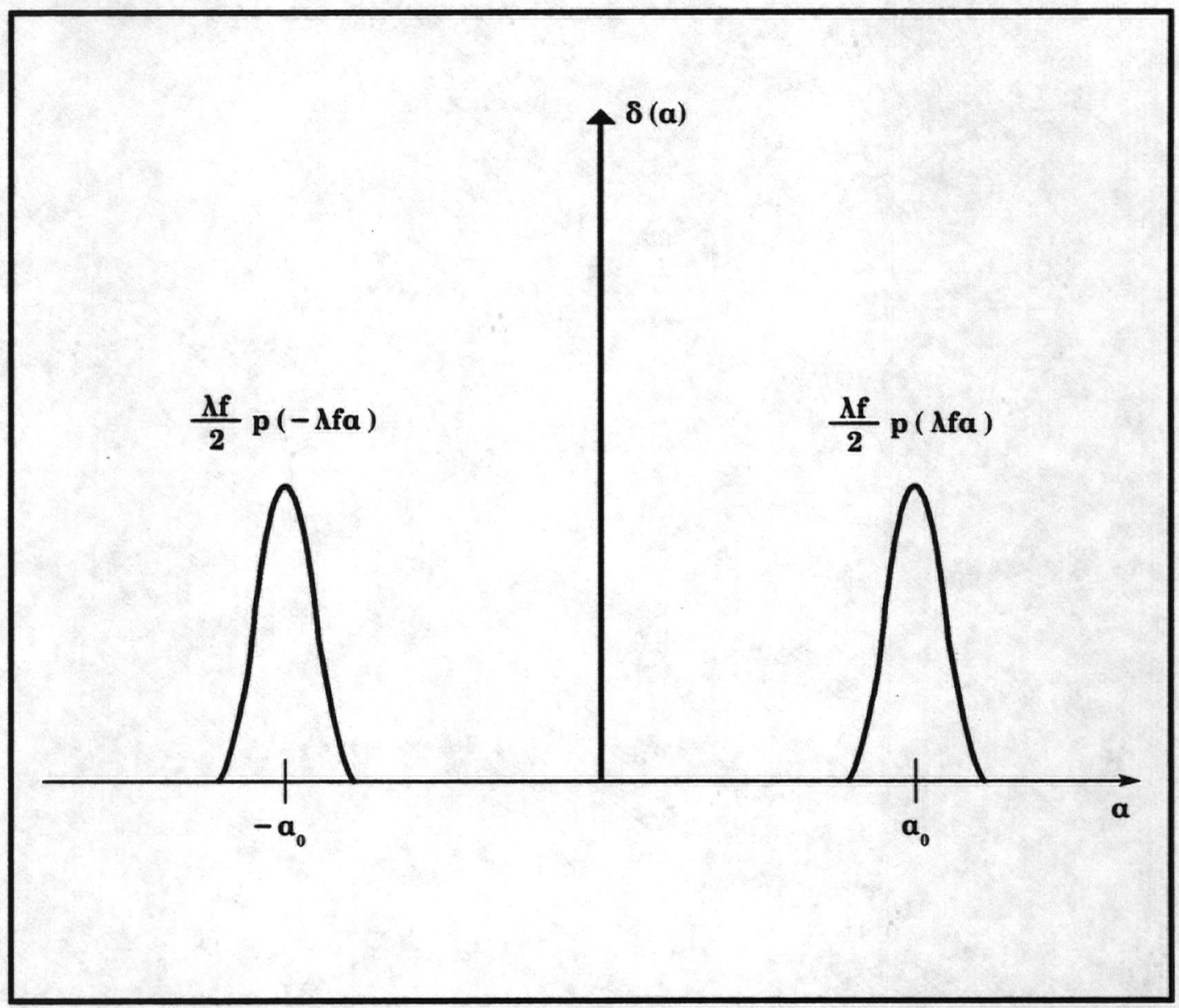

Fig. 3.3. Plot of $\bar{F}(a)$ vs. $a$ (the transform variable). This plot illustrates the final step in obtaining the density function $p(\cdot)$ from the intensity data measured in the transform plane of a doubleexposure far-field hologram. 
outlined above. A computer simulation was carried out to illustrate this method of measuring particle displacements; the results obtained are presented in Figs. $3.4-3.8$ and are discussed below.

A plot of the theoretical intensity profile in the transform plane of a double-exposure hologram is shown in Fig. 3.4a. In obtaining the plot of the intensity profile, it was assumed that the density function describing the particle displacements is Gaussian and that the particles are spherical in shape. Hence, the intensity in the transform plane is described by Equation (3.20) which is shown below for the special case of spherical particles:

$$
<I_{F}(u, v)>=\pi a^{2}\left[\frac{2 J_{1}\left(\frac{2 \pi a \rho}{\lambda f}\right)}{\frac{2 \pi a \rho}{\lambda f}}\right]\left\{1+\exp \left[-\frac{2 \pi^{2} \sigma^{2} u^{2}}{\lambda^{2} f^{2}}\right] \cos \left(\frac{2 \pi \mu u}{\lambda f}\right)\right\}
$$

As usual, $J_{1}(\cdot)$ denotes a Bessel function of the first kind and of order one and $a$ denotes the particle radius. The zone lens term was not included in the above expression since it is assumed that the object-to-hologram separation is large enough to effectively eliminate the zone lens if in-line far-field holography is to be used. The values selected for the various parameters in the above expression are:

$$
\begin{aligned}
& 2 a \text { (particle diameter) }=45 \mu, \\
& \mu \text { (mean displacement) }=365 \mu, \\
& \sigma \text { (standard deviation of displacement) }=60 \mu, \\
& \lambda \text { (wavelength) }=5145 \AA, \\
& f \text { (focal length of transform lens) }=0.5 \mathrm{~m} .
\end{aligned}
$$



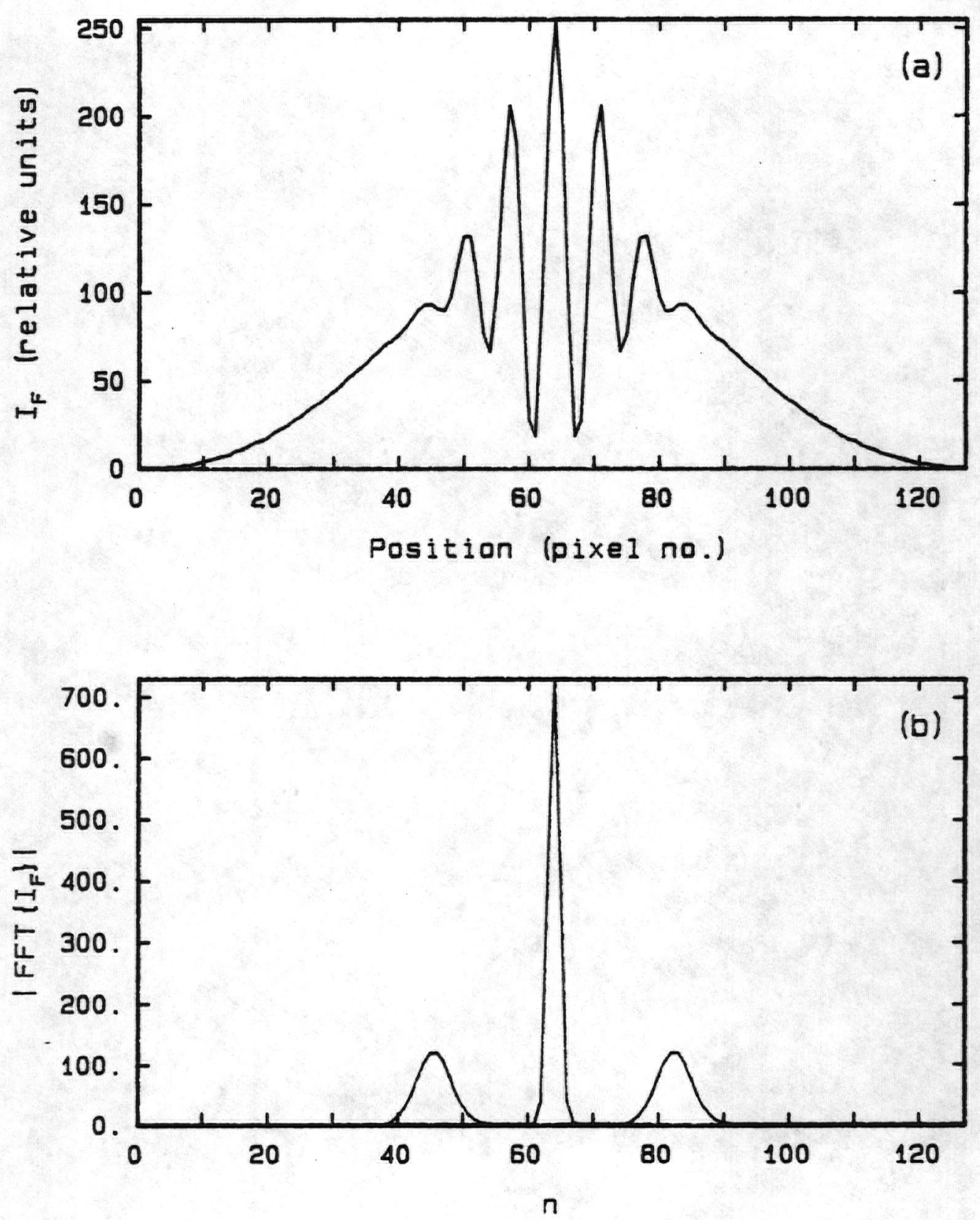

Fig. 3.4. (a) Plot of the theoretical intensity profile in the transform plane of a double-exposure hologram. (b) Magnitude of the FFT of the intensity profile vs. discrete transform variable $n$. 

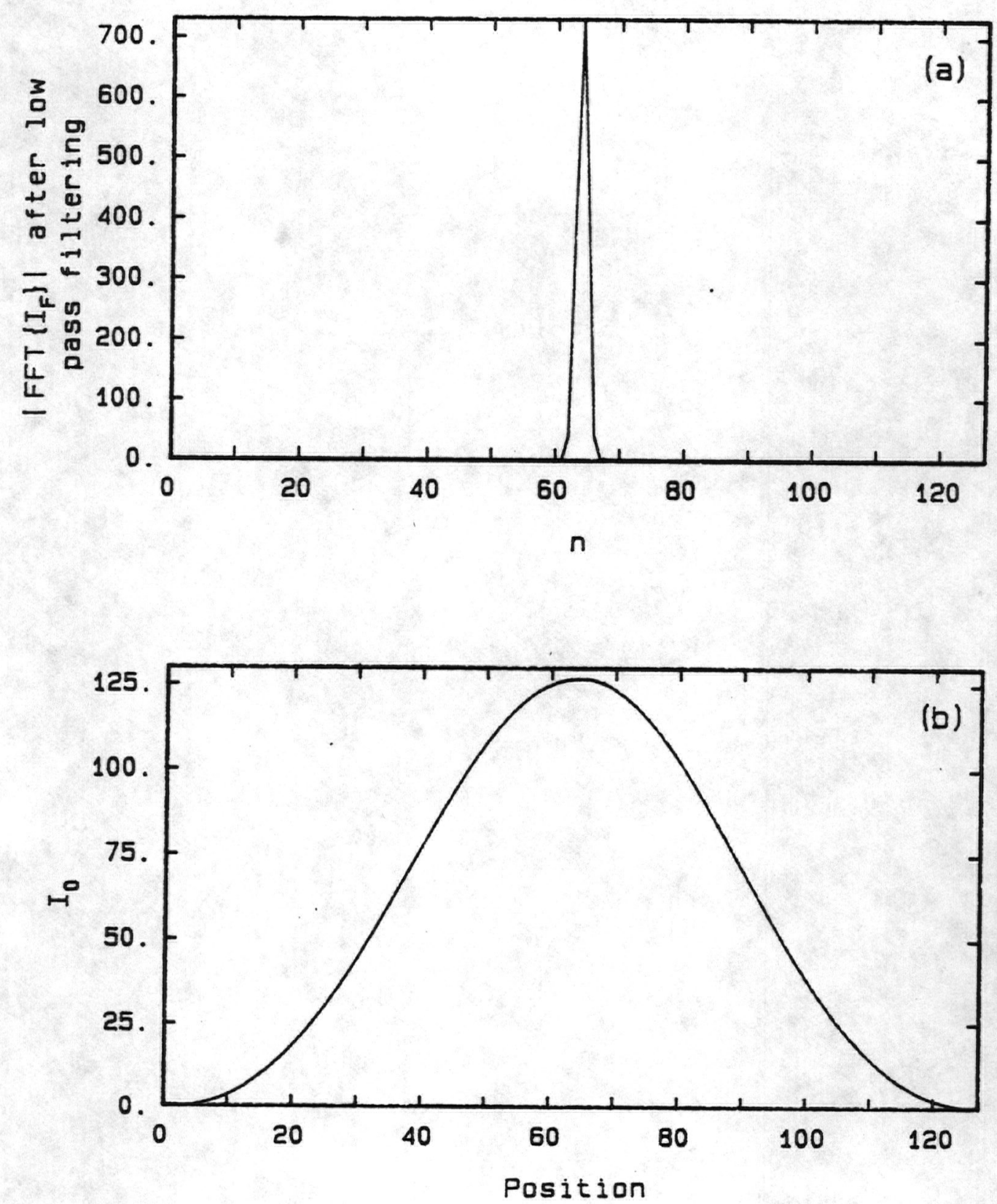

Fig. 3.5. (a) Plot of $\mid$ FFT $\left\{I_{F}\right\} \mid$ vs. $n$ after low pass filtering. (b) The envelope function $I_{0}$ is obtained by forming the inverse FFT of the data in (a). 

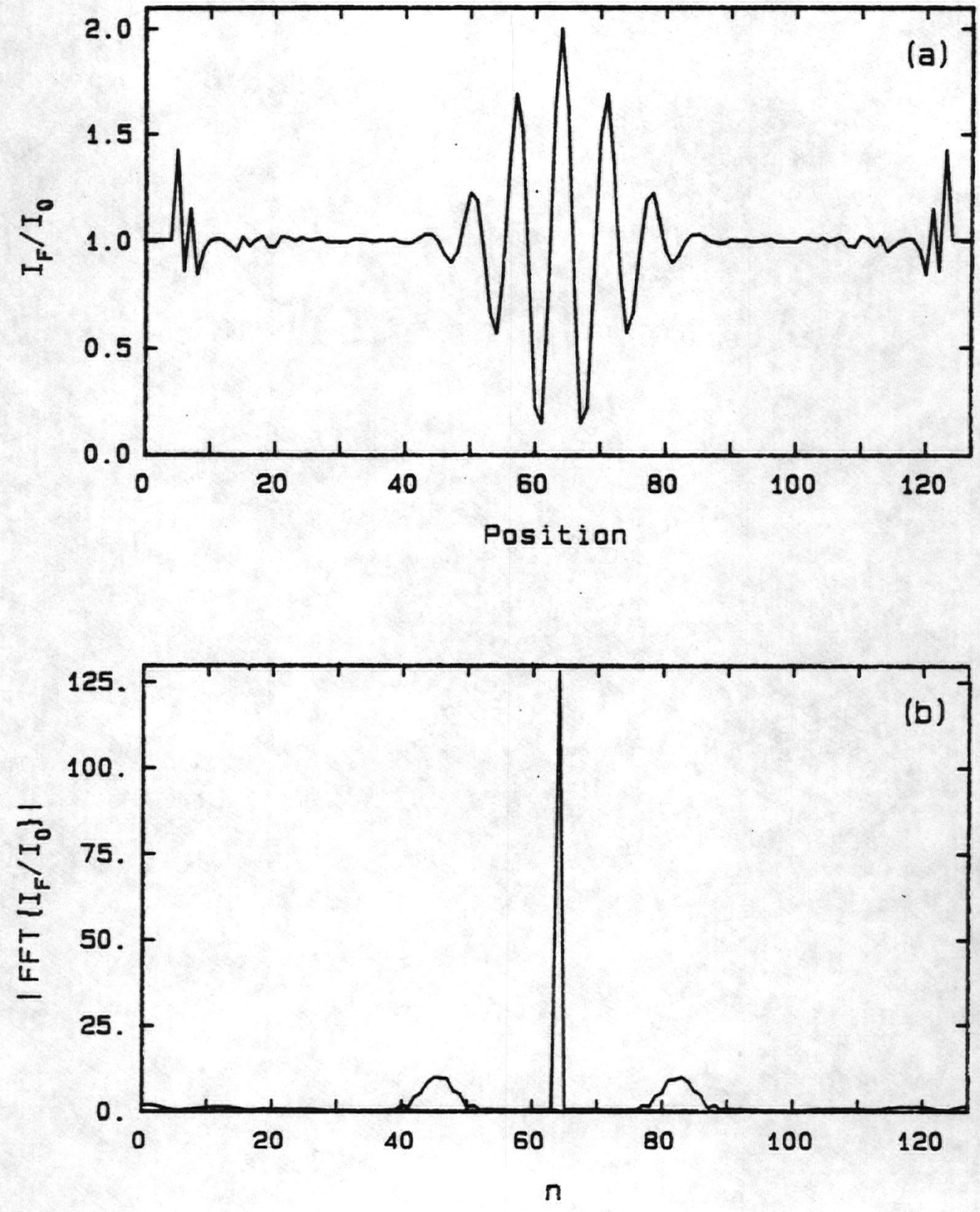

Fig. 3.6. (a) Plot of $I_{F} / I_{0}$ vs. position. The fluctuations near $n=$ 5 and $n=120$ are due to the fact that $I_{F}$ was assumed to be obtained with an 8-bit digitizer (see accompanying text). (b) Plot of |FFT $\left\{I_{F} / I_{0}\right\} \mid$ vs. discrete transform variable $n$. 

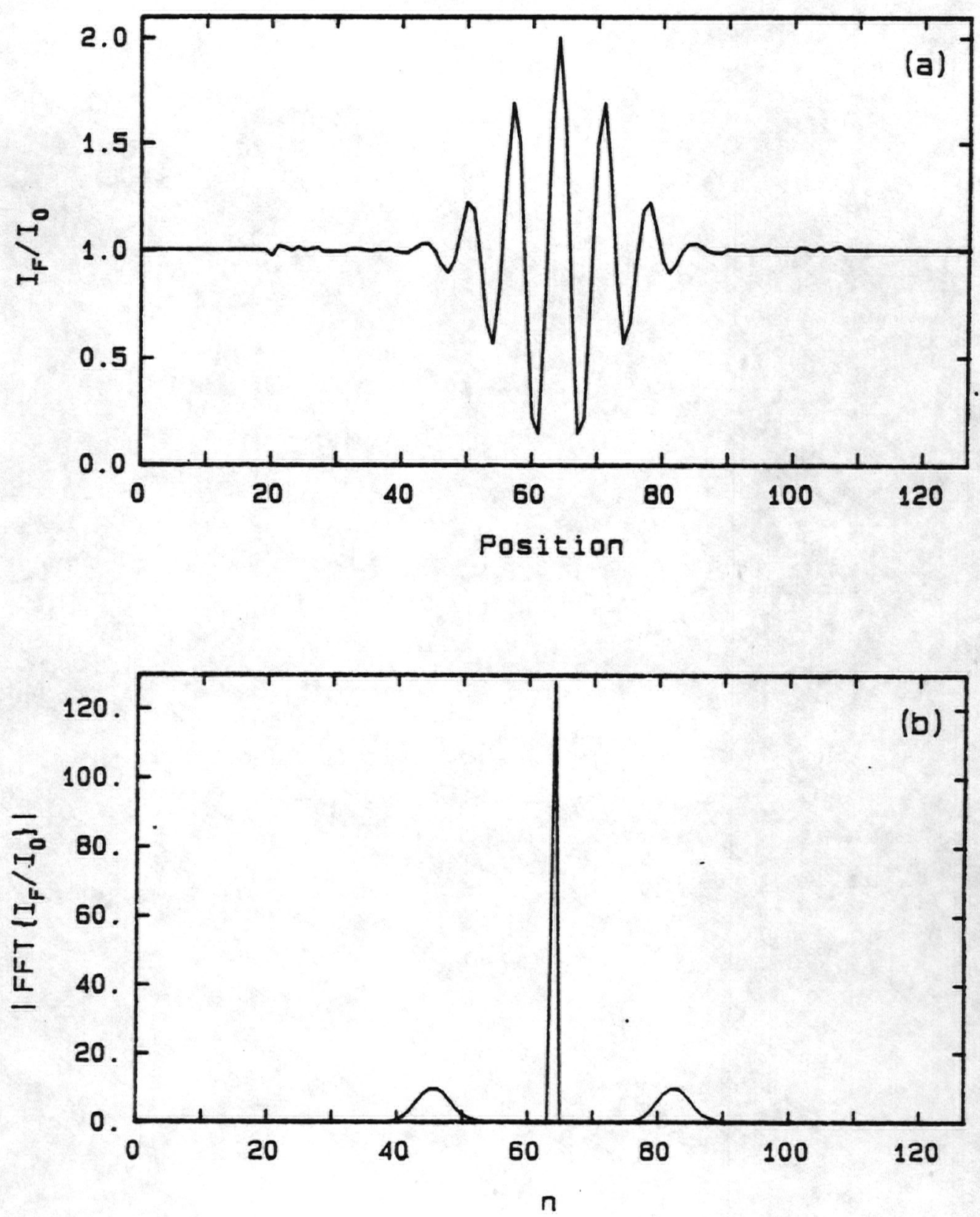

Fig. 3.7. (a) Same as Fig. 3.6a except the fluctuations near $n=5$ and $n=120$ were eliminated by setting $I_{F} / I_{0}$ to unity at these points. (b) Magnitude of the FFT of the curve in (a). 

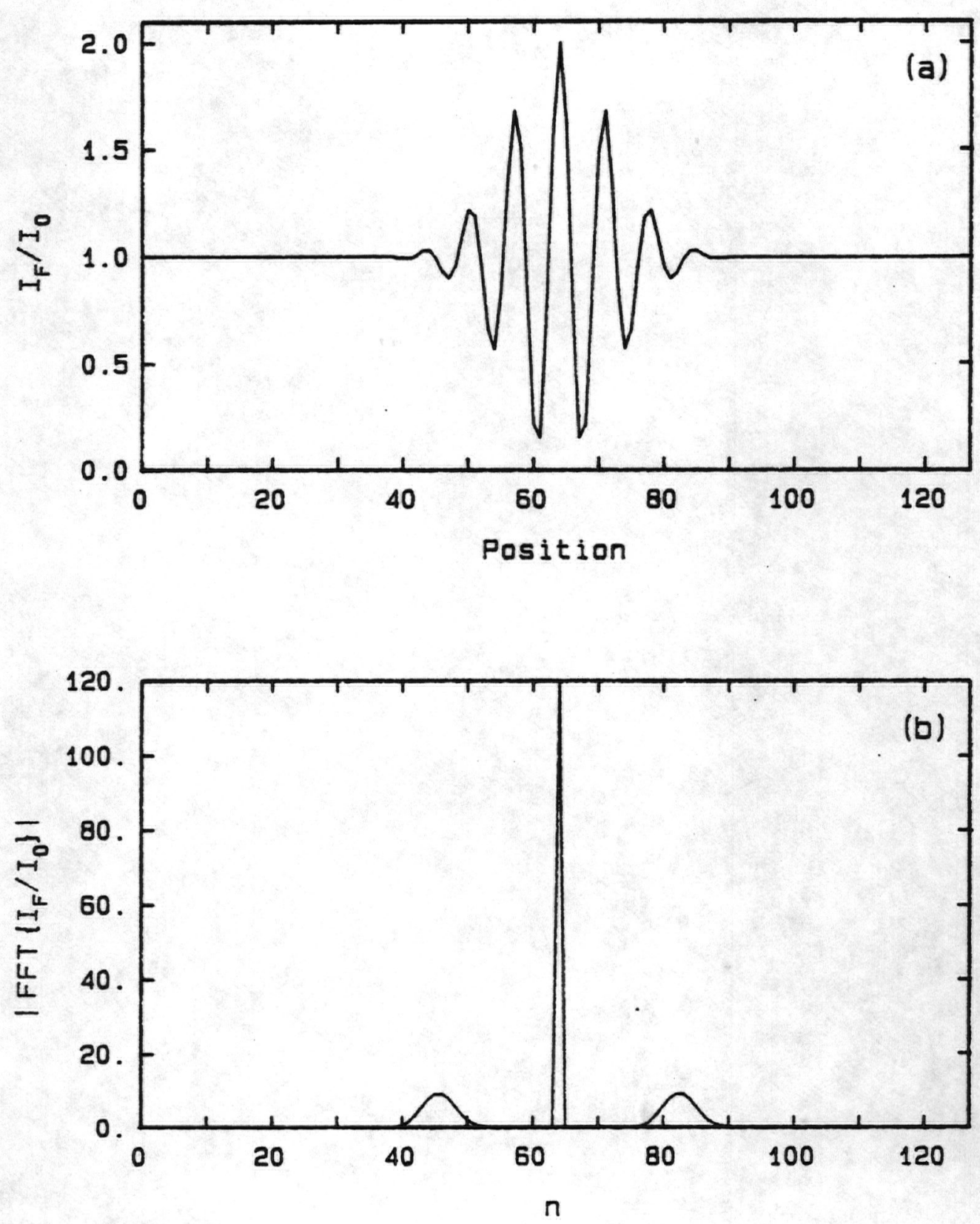

Fig. 3.8. (a) Theoretical plot of $I_{F} / I_{0}$. (b) Magnitude of the FFT of the curve in (a). 
In addition, the coordinate $v$ was assumed to be constant (specifically, $v=2 \mathrm{~mm}$ ) thereby simulating the results of a onedimensional scan taken at a distance of $2 \mathrm{~mm}$ from the origin of the transform plane. Figure 3.4 a was obtained by evaluating $\left\langle I_{F}(u, v)\right\rangle$ at 128 evenly spaced values between $u=-6.5 \mathrm{~mm}$ and $u=6.5 \mathrm{~mm}$ to simulate a measurement made by a 128-element array. It should be observed that the $u$-coordinate in Fig. $3.4 \mathrm{a}$ is specified by integer values which correspond to individual array elements (or pixels) to indicate that the intensity is sampled in a discrete fashion. Furthermore, the intensity measured by a given pixel will be digitized to 8 bits (i.e., 256 gray-levels) when the actual experiment is carried out. Hence, if all 256 gray-levels are utilized, the intensity measured by a given pixel will be represented by an integer ranging from 0 to 255 . The effects of the discrete intensity levels were incorporated into the computer simulation by scaling the calculated values of $\left\langle I_{F}(u, v)\right\rangle$ such that zero intensity is represented by the integer 0 and maximum intensity is represented by 255. By simulating the detector array and accounting for the discrete levels of intensity, the calculated intensity profile is similar in form to the data which would be obtained from an actual experiment. This set of data must then be processed to yield the density function that characterizes the particle displacements.

It should be recalled that the first step in obtaining the required density function is to divide the intensity profile by the 
envelope function (i.e., $I_{0}(\cdot)$ in Equation (3.27)). In this particular example, the envelope function is of the form $J_{1}(\cdot) /(\cdot)$ (see Equation (3.31)) thus, it may readily be removed from the intensity profile by division. However, the form of the envelope function is usually not known in advance; thus, a general method for obtaining it is clearly required. In fact, the envelope may be obtained directly from the measured intensity data by recognizing that the envelope itself is rather slowly varying while the term carrying the displacement information is rapidly varying. In other words, the envelope primarily consists of low frequency components whereas the term carrying the displacement information consists of relatively high frequency components. Hence, if the measured intensity profile is passed through a suitable low pass filter, the output would be the envelope function. The filtering operation is demonstrated in Figs. 3.4b - 3.5b. Clearly, the first step in the filtering operation is to evaluate the fast Fourier transform (FFT) of the intensity profile given in Fig. 3.4a; Fig. $3.4 \mathrm{~b}$ shows the magnitude of the FFT as a function of the discrete transform variable $n$. The origin of the frequency axis in Fig. $3.4 \mathrm{~b}$ was shifted from $n=0$ to $n=64$ to produce a symmetrical plot, hence, the low frequency information is centered about the point $n=64$. The filtering is performed by setting the magnitude of the FFT to zero for all points except those in an interval about $n=64$; the values of the FFT within this interval remain unchanged (see Fig. $3.5 \mathrm{a}$ ). The inverse 
transform of the filtered data is then conputed, yielding the envelope function (Fig. 3.5b).

As mentioned, once the envelope (denoted by $I_{0}$ ) is obtained, the next step in determining the required density function is to compute $I_{F} / I_{0}$ where $I_{F}$ represents the measured intensity profile. The result of performing this division is shown in Fig. 3.6a. When $I_{0}$ is zero (in the vicinity of $n=0$ and $n=127$ in Fig. 3.5a) the quantity $I_{F} / I_{0}$ clearly cannot be evaluated; instead, it is arbitrarily set to unity as shown in Fig. 3.6a. Furthermore, in regions where both $I_{F}$ and $I_{0}$ assume small, non-zero values, a small oscillation in $I_{F}$ will be greatly amplified when the quantity $I_{F} / I_{0}$ is computed. Thus, the plot of $I_{F} / I_{0}$ vs. position (Fig. 3.6a) exhibits rather large fluctuations near $n=5$ and $n=120$. The fluctuations in $I_{F}$ can be attributed to the fact that the intensity levels were assumed to be discrete. It should be recalled that as a result of digitization, the intensity $I_{F}$ is represented by an integer ranging from 0 to 255. Thus, if the actual intensity registered 1.4 on a scale of 0 to 255 , after digitization it would be rounded to a value of 1 which corresponds to a relative error of $29 \%$. However, only a small relative error is introduced at larger intensites. The net result, of course, is that $I_{F} / I_{0}$ exhibits large fluctuations in regions where $I_{F}$ and $I_{0}$ assume small values.

When the FFT of $I_{F} / I_{0}$ is computed to obtain the density function (it should be remembered that the transforming operation is the last step in obtaining the required density function from the 
intensity data), the fluctuations present in the function $I_{F} / I_{0}$ do, indeed, have an effect on the measured density function (see Fig. 3.6b). It is observed from Fig. $3.6 \mathrm{~b}$ that the portions of the curve corresponding to the density function do not exhibit a smooth, - Gaussian shape as expected. However, if the large fluctuations near $n=5$ and $n=120$ in Fig. 3.6 a are eliminated by setting the values of $I_{F} / I_{0}$ to unity at these points (see Fig. $3.7 \mathrm{a}$ ), the resulting FFT does yield the expected result (see Fig. 3.7b). For comparison, a theoretical plot of $I_{F} / I_{0}$ is given in Fig. 3.8a. This curve was obtained by plotting the term carrying the particle displacement information (i.e., the term in braces in Equation (3.31)) over the same interval as in Fig. 3.4a. The magnitude of the corresponding FFT is given in Fig. 3.8b. It is observed that there is good agreement between Figs. $3.7 \mathrm{~b}$ and $3.8 \mathrm{~b}$; however, it is necessary to eliminate the large fluctuations introduced when $I_{F} / I_{0}$ is computed in order to obtain good results. In practice, there is no problem with eliminating these fluctuations provided that the width of the envelope function (which is the Fourier transform of a typical particle's cross-sectional shape) exceeds the width of the Fourier transform of the density function.

\subsection{The effects of a limited number of particles}

The method outlined in the previous section for obtaining the density function was based on the assumption that the sample under study consists of a large number of particles; that is, large enough 
such that the summation of cosines in Equation (3.6) can be replaced by an integral expression (see Equations (3.7) - (3.13)). Unfortunately, it is difficult in general to determine the number of particles required before the summation can accurately be replaced by the integral. However, if a specific distribution of particle displacements is assumed, the value of the summation term may be computed for various values of $N$ (the number of particles) and the results can then be compared to the corresponding result obtained by evaluating the integral expression. Even though the results obtained in this manner are not valid the in general case, they can provide some insight on the ability to measure displacement information when a limited number of particles are present.

An example to illustrate the effects of a limited number of particles is presented; in particular, it is assumed that the particle displacements are one-dimensional and described by a Gaussian density function. As mentioned, the summation term in Equation (3.6) is the quantity of interest since it contains the displacement information. For the one-dimensional case, the summation term reduces to

$$
I^{\prime}{ }_{N}(u)=1+\frac{1}{N} \sum_{n=1}^{N} \cos \left(\frac{2 \pi u \Delta \xi_{n}}{\lambda f}\right)
$$

where a factor of $2 N$ was omitted for convenience. As the number of particles approaches infinity the above expression converges to (see Section 3.2) 


$$
I^{\prime}(u)=1+\int_{-\infty}^{+\infty} p\left(s_{1}\right) \cos \left(\frac{2 \pi u s_{1}}{\lambda f}\right) d s_{1}
$$

For the specific case where $p\left(s_{1}\right)$ is a Gaussian density function, this equation becomes

$$
I^{\prime}(u)=1+\exp \left[-\frac{2 \pi^{2} \sigma^{2} u^{2}}{\lambda^{2} f^{2}}\right] \cos \left(\frac{2 \pi \mu u}{\lambda f}\right)
$$

It should be recalled that a plot of the above expression was given in the previous section (Fig. 3.8a). The Fourier transform of Equation (3.34) yields the required density function, thus the FFT of the function shown in Fig. $3.8 \mathrm{a}$ was evaluated and is given in Fig. 3.8b. The plots shown in Figs. $3.8 \mathrm{a}$ and $\mathrm{b}$ represent results which occur in the limiting case as $N$ approaches infinity. For finite values of $N$, Equation (3.32) may be evaluated and the results obtained may then be compared to those obtained in the limiting case, thereby giving some indication as to when the summation may be replaced by the integral. Fig. $3.9 \mathrm{a}$ shows $I^{\prime}{ }_{N}$ plotted as function of position for $N=100$. In calculating $I_{N}^{\prime}$, a Gaussian random number generator was used to determine the individual particle displacements, $\Delta \xi_{n}$. The remaining parameters required to evaluate $I_{N}^{\prime}$ (i.e., $\sigma, \mu, \lambda$, and $f$ ) were chosen to agree with those used in the previous section to obtain Fig. 3.8. The FFT of $I_{N}^{\prime}$ was then calculated and a plot of $\mid$ FFT $\left\{I^{\prime}{ }_{N}\right\} \mid$ vs. the transform variable, $n$, is given in Fig. 3.9b. Plots of $I_{N}^{\prime}$ vs. position for 

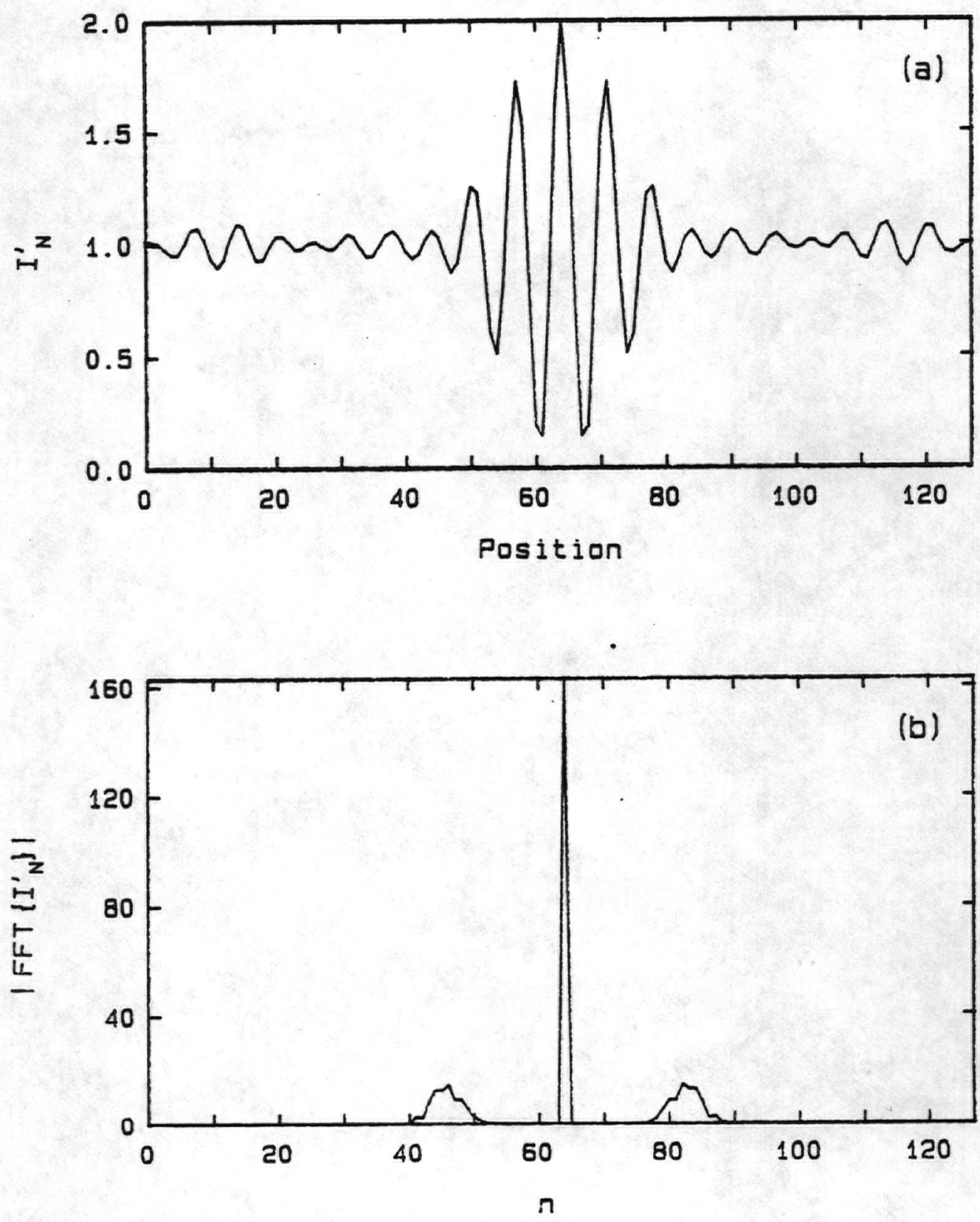

Fig. 3.9. (a) Plot of $I^{\prime}, v$ vs. Position for $N=100$. (b) $\mid$ FFT $\left\{I^{\prime}{ }_{N}\right\} \mid$ vs. $n$ (the transform variable). 

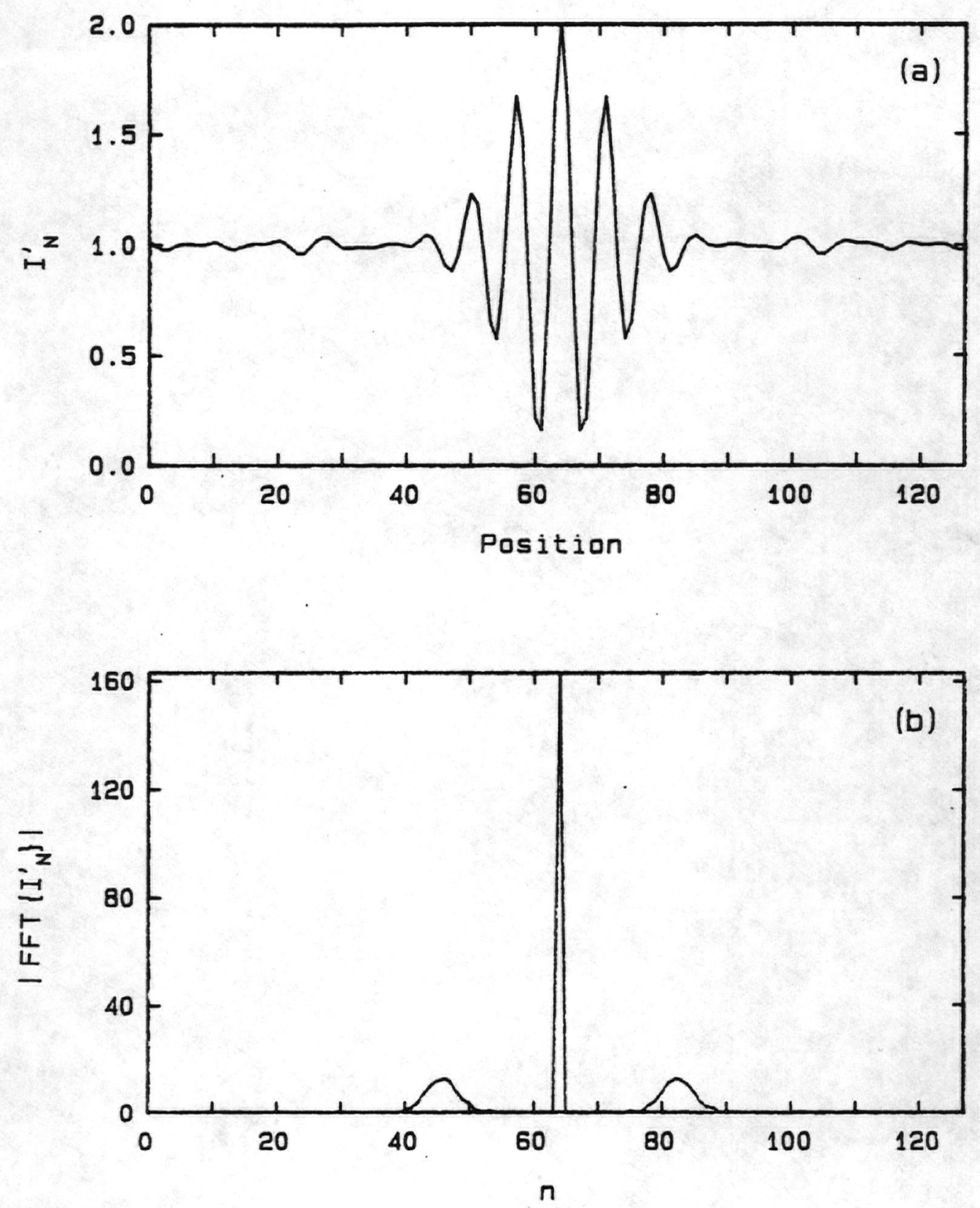

Fig. 3.10. (a) Plot of $I^{\prime}{ }_{\text {V }}$ vs. Position for $N=500$. (b) $\left|\operatorname{FFT}\left\{I^{\prime}{ }_{N}\right\}\right|$ vs. $n$. 

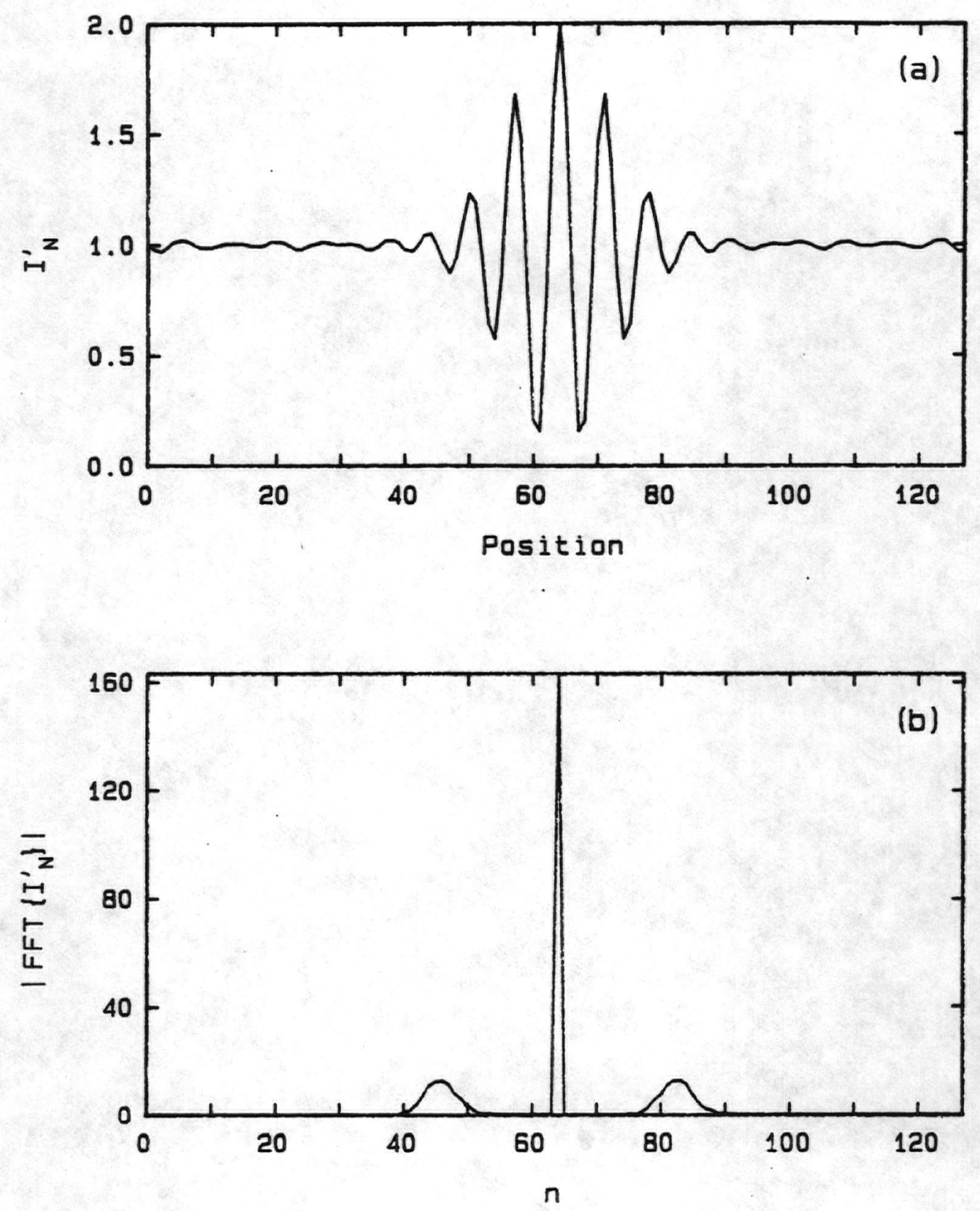

Fig. 3.11. (a) Plot of $I^{\prime}{ }_{N}$ vs. Position for $N=1000$. (b) $\left|\operatorname{FFT}\left\{I^{\prime},{ }^{\prime}\right\}\right|$ vs. $n$. 
$N=500$ and $N=1000$ are given in Fig. 3.10 and 3.11 , respectively (the corresponding FFT's are also included). Upon comparing Figs. 3.9 - 3.11 with 3.8 , it is observed that once the sample size approaches 500 particles, Equation (3.34) becomes a good approximation to Equation (3.32).

3.7 Measuring two-dimensional velocity and displacement distributions in the transform plane of a double-exposure hologram

If the particles of interest are characterized by a twodimensional distribution of displacements (where the particle motion is confined to a plane parallel to the hologram plane), the displacement information is displayed in the optical transform plane in accordance with Equation (3.11). Thus, it appears that a twodimensional distribution of displacements may be measured by an appropriate generalization of the one-dimensional data processing method described in Section 3.5. However, as the following analysis will show, there are difficulties associated with obtaining the twodimensional density function in this manner.

As mentioned, the intensity of the transform plane of a double-exposure far-field hologram is given by Equation (3.11). In analogy with the procedure for obtaining a one-dimensional distribution of displacements, the envelope function $I_{0}(u, v)$ is first removed by division yielding

$$
I^{\prime}(u, v)=\frac{<I_{F}(u, v)>}{I_{0}(u, v)}=1+\iint_{-\infty}^{+\infty} p\left(s_{1}, s_{2}\right) \cos \left[\frac{2 \pi}{\lambda f}\left(u s_{1}+v s_{2}\right)\right] d s_{1} d s_{2}
$$


To obtain the required density function, the two-dimensional Fourier transform of the above expression is then evaluated; i.e.,

$$
\begin{aligned}
F\left\{I^{\prime}(u, v)\right\} & =\iint_{-\infty}^{+\infty} I^{\prime}(u, v) \exp [-i 2 \pi(a u+\beta v)] d u d v \\
& =\delta(\alpha, \beta)+\frac{(\lambda f)^{2}}{2} p(-\lambda f a,-\lambda f \beta)+\frac{(\lambda f)^{2}}{2} p(\lambda f a, \lambda f \beta)
\end{aligned}
$$

where $a$ and $\beta$ are the transform variables. Hence, if $\left\langle I_{F}(u, v)\right\rangle$ is known, the density function that characterizes the particle displacements may be determined. However, the quantity $\left\langle I_{F}(u, v)\right\rangle$ does not represent the expected value of intensity at all points in the transform plane; it should be remembered that there will be an intense distribution of light near the origin due to light that is undiffracted by the hologram. In practice, the undiffracted light reduces the information available in the transform plane in two respects: First, the intensity near the origin is relatively large, causing the corresponding detector elements to saturate. Second, light scattered from the region about the origin to other portions of the transform plane tends to reduce the contrast of the fringes which contain the particle displacement information. Thus, it is usually necessary to use a stop in the transform plane to block out the intense light in the vicinity of the origin. As a result, the quantity $I^{\prime}(u, v)$ in Equation (3.35) is not available in practice; the quantity actually obtained is more closely represented by 


$$
I_{a c t}^{\prime}(u, v)=\left[1-\operatorname{circ} \frac{\rho}{r_{0}}\right] I^{\prime}(u, v),
$$

where $\rho^{2}=u^{2}+v^{2}$ and $r_{0}$ is the width of the stop. Evaluating the Fourier transform of the above expression in an attempt to obtain the required density function yields

$$
F\left\{I_{a c t}^{\prime}(u, v)\right\}=\left[\delta(a, \beta)-\pi r_{0}^{2} \frac{2 J_{1}\left(2 \pi\left[a^{2}+\left.\beta^{2}\right|^{1 / 2} r_{0}\right)\right.}{2 \pi\left[a^{2}+\beta^{2}\right]^{1 / 2} r_{0}} \mid * F\left\{I^{\prime}(u, v)\right\}\right.
$$

where * deontes a convolution and $F\left\{I^{\prime}(u, v)\right\}$ is given in Equation (3.36). Hence, the particle displacement information is somewhat obscured as a result of the convolution.

A computer simulation was carried out to better illustrate the consequences of using a stop to block out the undiffracted light. In performing the simulation, it was assumed that the random variables, $\Delta \xi_{n}$ and $\Delta \eta_{n}$ (which describe the displacement of the $n^{\text {th }}$ particle), are jointly normal and independent. Thus, the intensity in the transform plane of the corresponding far-field hologram is given by Equation (3.22) for the ideal case with no stop present. (The stop may be included in the manner indicated in Equation (3.37)). Dividing both sides of Equation (3.22) by the envelope $I_{0}(u, v)$ yields

$$
I^{\prime}(u, v)=1+\exp \left[-\frac{2 \pi^{2}}{\lambda^{2} f^{2}}\left(\sigma_{1}^{2} u^{2}+\sigma_{2}^{2} v^{2}\right)\right] \cos \left[\frac{2 \pi}{\lambda f}\left(\mu_{1} u+\mu_{2} v\right)\right]
$$


The values used for the various parameters in the above expression are given below:

$$
\begin{aligned}
& \sigma_{1}=\sigma_{2}=45 \mu, \\
& \mu_{1}=700 \mu, \\
& \mu_{2}=0, \\
& \lambda=5145 \AA, \\
& f=0.5 \mathrm{~m} .
\end{aligned}
$$

To simulate a $128 \times 128$-element detector array, $I^{\prime}(u, v)$ was calculated at the appropriate points in the following region of the $u, v-p l a n e:$ $-6.7 \mathrm{~mm}<u<6.7 \mathrm{~mm},-6.7 \mathrm{~mm}<v<6.7 \mathrm{~mm}$. The sampled version of $I^{\prime}(u, v)$ is denoted by $I_{k l}^{\prime}$. Computing the two-dimensional FFT of $I_{k l}^{\prime}$ will clearly yield the desired density function if no stop is present (see Fig. 3.12a). If a stop having a diameter of $1.2 \mathrm{~mm}$ is used, the function shown in Fig. $3.12 \mathrm{~b}$ is obtained instead of the desired density function. An additional comparison between the results obtained is given in Fig. 3.13 which shows an axial slice of the two-dimensional FFT of $I_{k l}^{\prime}$. It is observed that the twodimensional "density function" which is computed from the available intensity data is quite different from the actual density function. However, it will be shown in the next section that the twodimensional density function can be accurately determined if the horizontal and vertical components of displacement are statistically independent. 

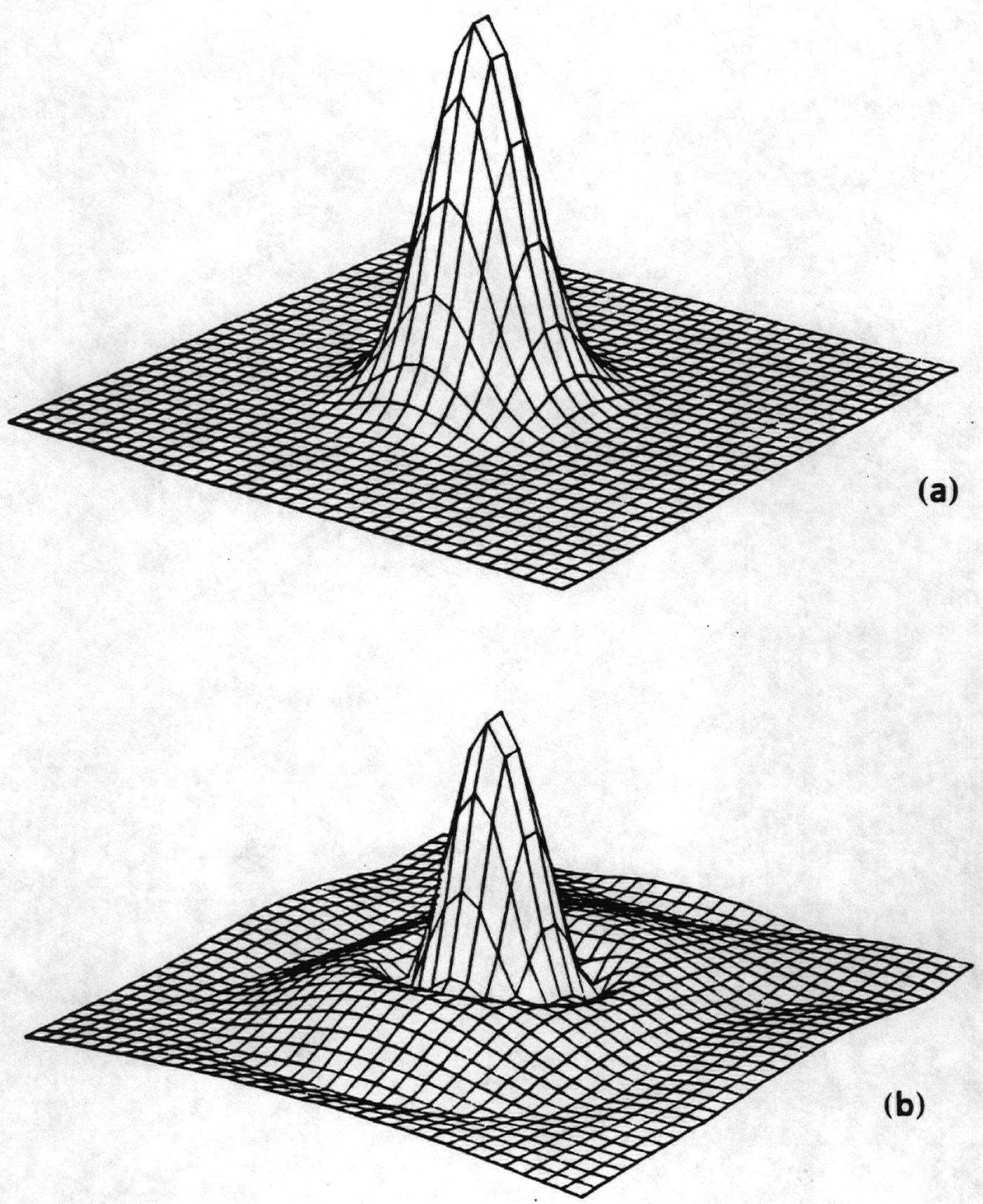

Fig. 3.12. (a) Two-dimensional density function obtained by a computer simulation assuming no stop is present in the optical transform plane. (b) The "density function" obtained when the stop is present. 


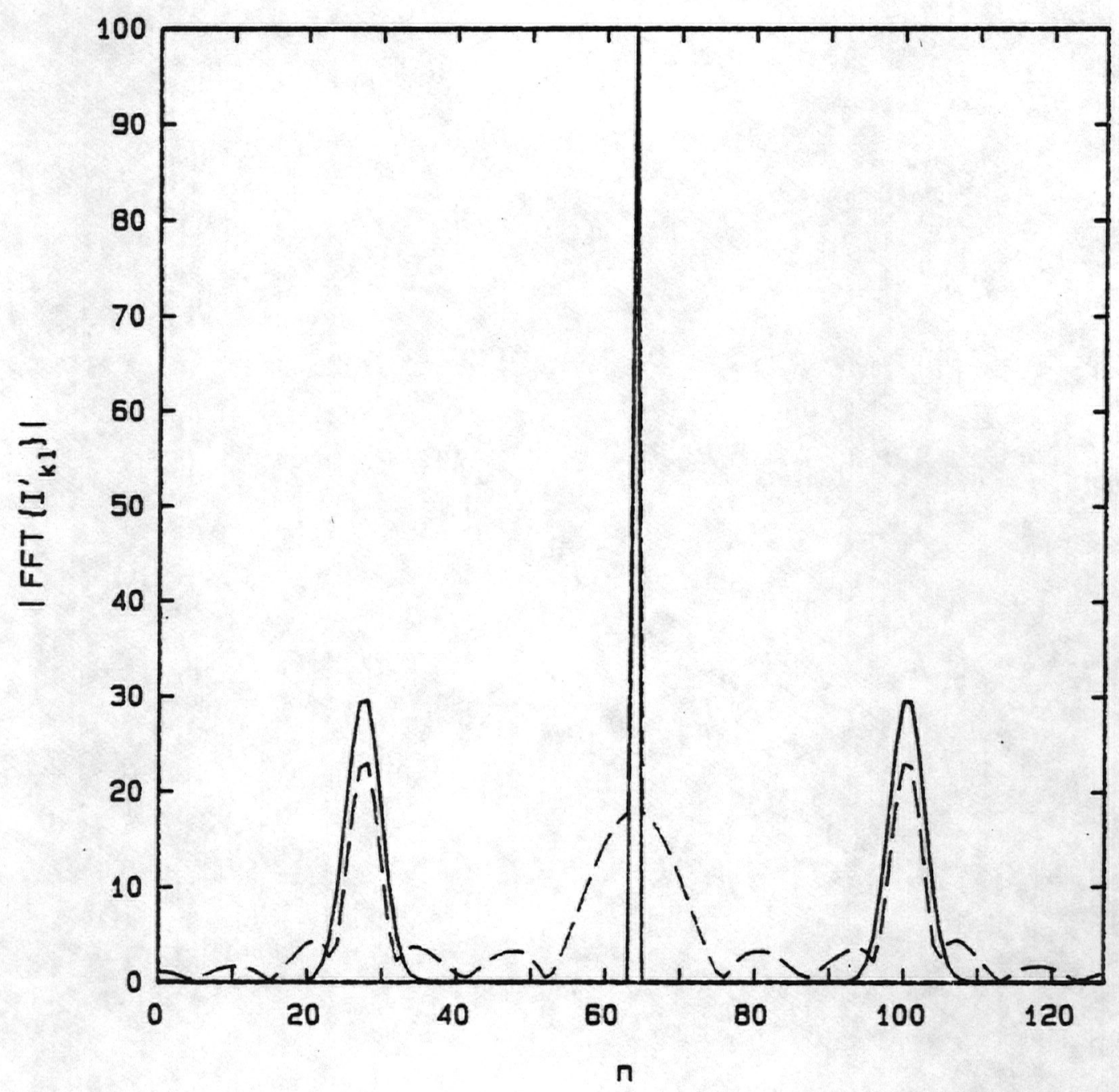

Fig. 3.13. Plot of an axial slice of the two-dimensional FFT of $I_{k l}^{\prime}$. The solid curve illustrates the result obtained in the ideal case assuming no stop is present in the optical transform plane. The dotted curve illustrates the result obtained with the stop present. 
3.8 Measuring two-dimensional velocity and displacement distributions when the horizontal and vertical components of displacement are independent

If the horizontal and vertical components of particle displacement are statistically independent, the corresponding joint density function may be expressed as a product of the marginal density functions (Davenport and Root [1958]), i.e.

$$
p\left(s_{1}, s_{2}\right)=p_{1}\left(s_{1}\right) p_{2}\left(s_{2}\right)
$$

Substituting the above result into the expression for the intensity in the transform plane of a doubly exposed far-field hologram (Equation (3.11)) yields, after dividing both sides of Equation (3.11) by $I_{0}(u, v)$,

$$
I^{\prime}(u, v)=1+\iint_{-\infty}^{+\infty} p_{1}\left(s_{1}\right) p_{2}\left(s_{2}\right) \cos \left[\frac{2 \pi}{\lambda f}\left(u s_{1}+v s_{2}\right)\right] d s_{1} d s_{2} .
$$

If the intensity pattern is sampled along a line parallel to the $u$ axis and at a distance $v_{0}$ from the origin, the above expression becomes

$$
I^{\prime}\left(u, v_{0}\right)=1+\iint_{-\infty}^{+\infty} p_{1}\left(s_{1}\right) p_{2}\left(s_{2}\right) \cos \left[\frac{2 \pi}{\lambda f}\left(u s_{1}+v_{0} s_{2}\right)\right] d s_{1} d s_{2}
$$

(By scanning the intensity pattern away from the origin, the problems associated with blocking out the intense light near the origin are virtually eliminated.) Transforming the above equation with respect to the variable $u$ yields 


$$
F\left\{I^{\prime}\left(u, v_{0}\right)\right\}=\delta(a)+\frac{\lambda f}{2}\left[p_{1}(-\lambda f a)+p_{1}(\lambda f a)\right] \int_{-\infty}^{+\infty} p_{2}\left(s_{2}\right) \cos \frac{2 \pi v_{0} s_{2}}{\lambda f} d s_{2},
$$

where $a$ is the transform variable. Since $v_{0}$ is a constant, the integral in Equation (3.43) evaluates to a constant. Thus, the above equation indicates that the marginal density function $p_{1}(\cdot)$ may be determined (to within a constant factor) from data obtained in a one-dimensional scan of the intensity pattern. The ambiguity introduced by the unknown constant factor may be resolved by using the fact that the area under the density function is equal to unity by definition.

In a similar fashion, $p_{2}(\cdot)$ may then be determined by procesing the data obtained from a scan parallel to the $v$-axis at a distance $u_{0}$ from the origin. Hence, when the horizontal and vertical components of particle displacement are statistically independent, determining the joint density function reduces to the problem of obtaining two, one-dimensional density functions.

\subsection{Summary}

The theoretical aspects of double-exposure holograms and the corresponding Fourier transforms were examined. It was shown that the individual particle displacements are represented in the transform plane by a summation of cosinusoids. In particular, the frequency and orientation of a given cosinusoid is directly related to the magnitude and direction, respectively, of a given particle's 
displacement. The advantage of using the optical Fourier transform of the hologram, rather than the reconstructed images, is that the particle displacement information displayed in the transform plane is well suited for digital processing.

A procedure was developed for obtaining the displacement information from intensity data measured in the transform plane. It was shown that the probability density function that characterizes the particle displacements may be determined by: 1) Measuring the intensity in the transform plane of a double-exposure hologram, 2) Obtaining the particle-size dependent envelope from the measured intensity data by digital filtering, 3) Dividing the measured intensity profile by the envelope function, and 4 ) Computing the FFT of the quantity obtained by dividing the intensity profile by the envelope. Computer simulations were carried out, indicating that one-dimensional distributions may be measured successfully. Twodimensional distributions are difficult to measure in general, because a portion of the required intensity data is inaccessible as a result of the strong component of undiffracted light. However, it was shown that a two-dimensional density function can be accurately determined if the horizontal and vertical components of displacement are statistically independent.

Multiple-exposure holography may also be used for measuring particle displacements and velocities, and this topic is briefly discussed in Appendix B. 


\section{Chapter IV}

\section{Measuring Particle Displacements in the Transform Plane of a Double-Exposure Hologram: Experimental Results}

\subsection{Introduction}

In the previous chapter, the theoretical aspects of doubly exposed holograms were examined. A method was then developed for obtaining the displacement information from intensity data measured in the transform plane. In this chapter, experimentally obtained results are presented, indicating that the procedure described in Chapter 3 may indeed be used in practice to determine particle displacement information from doubly exposed holograms.

\subsection{Data acquisition}

Since the intensity data in the transform plane of a doubleexposure hologram are to be processed digitally, this set of data must be made available to a computer. Thus, a system consisting of a TV camera, video recorder, image digitizer and a computer was used for the data acquisition (see Fig. 4.1).

As shown in Fig. 4.1, acquiring the data is a two-step process. First, the optical Fourier transform of a double-exposure hologram is detected by a TV camera and the output of the camera is recorded on video tape (see Fig. 4.1a). In the second step (Fig. 

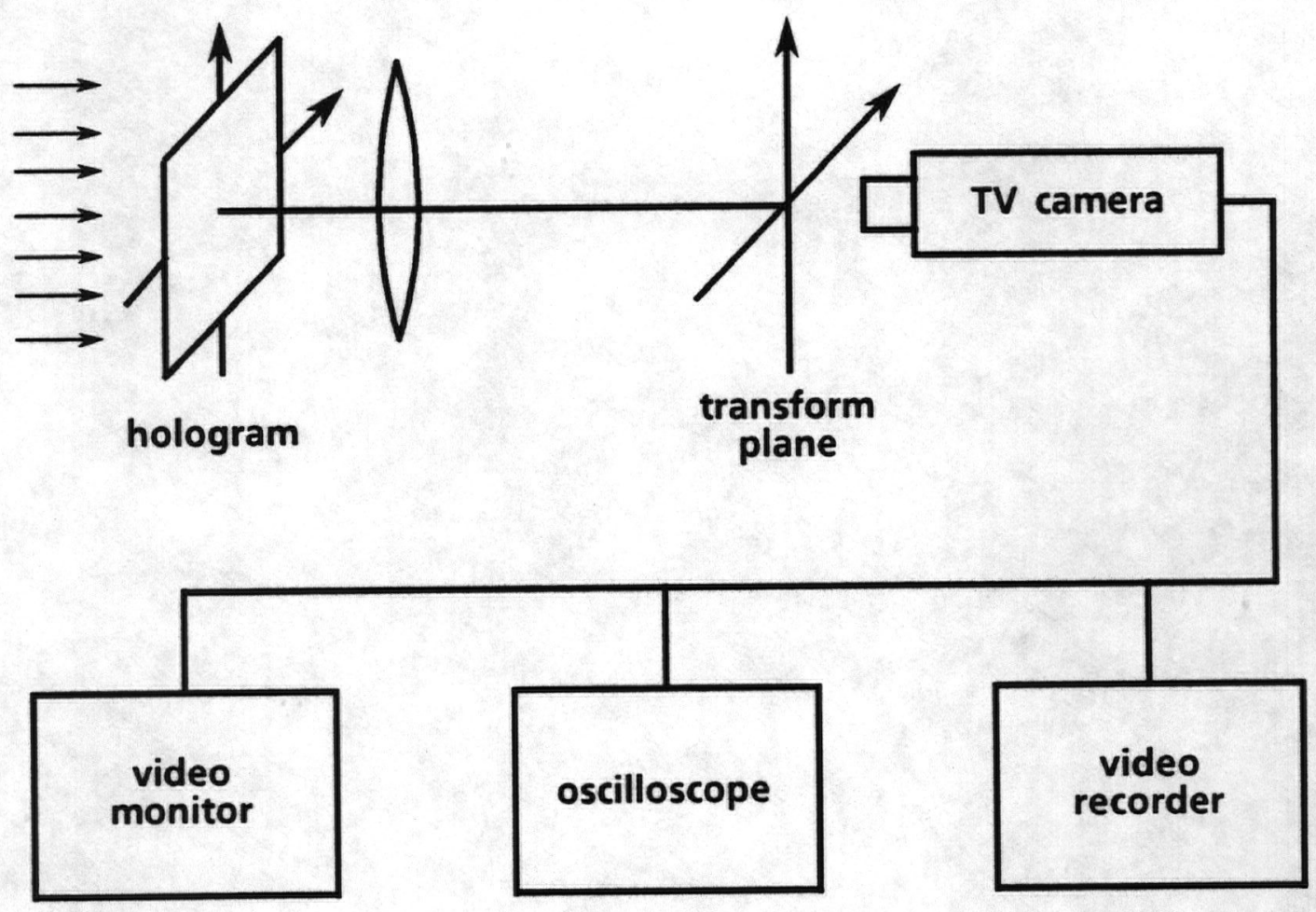

(a)

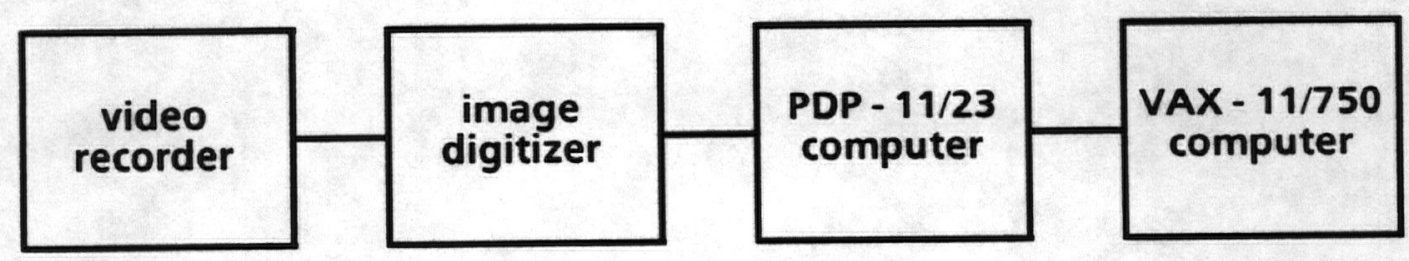

(b)

Fig. 4.1. Optical-digital system used for data acquisiton. (a) System for recording and monitoring the video signal. (b) Interfacing the video signal to the computer. 
$4.1 \mathrm{~b})$, the recorded video signal is digitized and is then sent to a computer which performs the required digital processing. The video recorder can, of course, be eliminated by connecting the output of the TV camera directly to the digitizer. However, the digitizer was located in a separate laboratory, making it more convenient to use the video recorder rather than a direct cable between the TV camera and digitizer.

\subsubsection{The data acquisition system in detail}

The previous section presented an overview of the data acquisition system; a more complete description of the individual system components is given below.

An MTI Series 68 instrumentation grade camera (manufactured by Dage/MTI, Inc.) equipped with a Newvicon image tube was used to detect the optical Fourier transform pattern of the doubly exposed hologram. The Newvicon tube was selected because it is better than a conventional vidicon when quantitative measurements are required. Namely, the gamma of the Newvicon is nearly unity and it exhibits minimal drift, thereby making calibration relatively easy. The output of the TV camera is a composite video signal which is fed directly to a JVC CR-6600V video cassette recorder.

An oscilloscope (Tektronix 2215) was used to monitor the camera output in addition to a standard video monitor (see Fig. 4.2a). The oscilloscope is particularly useful since it allows any horizontal line of the video field to be viewed. That is, the 
intensity profile corresponding to a single horizontal scan line is displayed directly on the screen. By observing this intensity profile, it is easy to determine whether or not the video signal is being clipped. If clipping occurs, the intensity of the laser used to illuminate the hologram must be decreased to an appropriate level. Furthermore, it is desirable to utilize the entire dynamic range of the video system. Thus, the laser intensity should ideally be set to produce a video signal whose peak amplitude is just below the clipping level. Clearly, the laser intensity may be properly adjusted by monitoring the video signal with the oscilloscope.

To make the video information available to the computer, the output signal from the video recorder is fed to an image digitizer (Video Frame Store 274D, manufactured by Colorado Video, Inc.). The digitizer converts a given frame of analog video information to a digitized image consisting of $512 \times 512$ pixels with 256 gray-levels (i.e., 8 bits per pixel). The digitized image is retained in the memory of the frame store and the computer (PDP-11/23) may then be used to access any desired pixel or group of pixels from the frame store. Once the data is acquired by the PDP-11/23 it is then sent to a VAX-11/750 which was programmed to perform the required processing -

4.3 Recording holograms with known particle displacements

It would be desirable to record a hologram of a particle sample for which the velocity distribution of the particles is known 
in advance since this hologram may then be used as a test input to the optical-digital system shown in Fig. 4.1. The particle displacement information determined by the hybrid system may be compared to the known displacements, thereby indicating whether or not the system is performing as expected. Unfortunately, it is difficult in practice to produce a double-exposure hologram of a dynamic sample for which the distribution of particle velocities is known a priori. As a result, a method for simulating doubleexposure holograms was developed so that the performance of the optical-digital system may be evaluated.

The first step in producing such a hologram was to plot a random array of dots using a graphics plotter operating under computer control. The random array consisted of dot-pairs which were uniformly distributed over the plotting area; the spacing between the dots comprising a given dot-pair was determined by a preselected random number generator. For example, to simulate a Gaussian distribution of particle displacements, a Gaussian random number generator would be used to determine the spacing between dots. A dot is formed each time the plotter's pen makes contact with the surface of the paper; hence, the thickness of the pen determines the diameter of the dot. In our case the dots were approximately $0.4 \mathrm{~mm}$ in diameter. The random array of dots was then photo-reduced to $1 / 10$ of its original size. As a result, the demagnified array measured $3.5 \mathrm{~cm}$ in diameter while a typical dot had a diameter of approximately $40 \mu \mathrm{m}$. The demagnified array was 
recorded onto a Kodak High Resolution Plate, Type $1 \mathrm{~A}$ which was subsequently developed using a reversal process. Thus a positive transparency of the array of dots is obtained. (A similar procedure for producing a random array of dots has recently been reported by Hinsch etal. [1984] in connection with the study of fringe visibility in speckle velocimetry.) The resulting black discs on the otherwise transparent plate form diffracting objects which simulate particles. A far-field hologram of the simulated particles may then be recorded to provide the required test input to the hybrid system shown in Fig. 4.1.

\subsubsection{Recording a transparency of the random array of dots}

As mentioned above, the random array of dots produced by the graphics plotter is recorded onto a photographic plate and developed using a reversal process. A detailed description of the recording and developing procedure is presented below.

The array of dots was photographed using a Polaroid MP-4 camera with a film holder modified to accept a $2^{\prime \prime} \times 2^{\prime \prime}$ glass plate. Kodak High Resolution Plates, Type 1A were selected because the high resolving power (greater than 2000 lines per $\mathrm{mm}$ ), high contrast and fine grain make them suitable for recording the small $(40 \mu \mathrm{m})$ dots that result when the original array is demagnified. After exposure, the plates were then processed according to the following procedure:

1. Develop in Kodak D-8

2. Rinse in water

3. Bleach in Kodak Bleach Bath $\mathrm{R}-9$
5 min.

$2 \min$.

2 min. 


$\begin{array}{lr}\text { 4. Rinse in water } & 1 \mathrm{~min} . \\ \text { 5. Clear in Kodak Clearing Bath CB-6 } & 3 \mathrm{~min} . \\ \text { 6. Rinse in water } & 4 \mathrm{~min} \text {. } \\ \text { 7. Presoak in Kodak HRP developer } & 30 \mathrm{sec} \text {. } \\ \text { 8. Reversal exposure ( } 30 \text { footcandles) } & 30 \mathrm{sec} . \\ \text { 9. Develop in Kodak HRP developer } & 5 \mathrm{~min} . \\ \text { 10. Fix in Kodak Rapid Fixer } & 2 \mathrm{~min} . \\ \text { 11. Rinse in Water } & 5 \mathrm{~min} .\end{array}$

(This is the recommended procedure for reversal processing of Kodak High Resolution plates as given by Kodak Publication No. P-47: KODAK High Resolution Plates.) Although the chemical baths and processing times 1 isted are specific to Kodak High Resolution Plates, the above procedure represents a rather general method for reversal processing of silver halide emulsions. Namely, the exposed plate is initially placed in a developer which produces a negative image. After development, the plate is immersed in a bleach bath that removes the negative image while leaving behind the unexposed grains of silver halide. The bleach bath typically contains potassium dichromate which imparts a yellow stain to the emulsion; this stain is removed by the clearing bath. The plate is then illuminated with white light to expose the remaining silver halide, consequently, a positive image is obtained upon re-developing the plate. 
4.4 Experimentally obtained measurements of one-dimensional displacement and velocity distributions

It should be recalled from Chapter 3 that the probability density function that characterizes the particle displacements may be determined by: 1) measuring the intensity in the transform plane of a double-exposure hologram, 2) obtaining the particle-sizedependent envelope from the measured intensity data by digital filtering, 3) dividing the measured intensity profile by the envelope function, and 4) computing the FFT of the quantity obtained by dividing the intensity profile by the envelope. In the following subsections, experimental results obtained by using this technique are presented. The first set of data (given in Section 4.4.1) is the result of using far-field holograms of random arrays of opaque discs as input to the hybrid processing system. As noted earlier, random arrays with known statistical properties were fabricated so that the system output may be compared with the known density function. The second set of data (given in Section 4.4.2) illustrates a real application of the holographic technique for measuring a distribution of particle velocities. In this particular example, the velocity distribution of $80 \mu \mathrm{m}$ particles falling in water is determined.

4.4.1 Results obtained by using holograms of random arrays of opaque discs as input to the hybrid processing system

Far-field holograms of the random arrays were recorded in the manner indicated in Fig. 1.1; in particular, the illumination was 
provided by an Argon-ion laser operating at $5145 \AA$ and the holograms were recorded onto Kodak High Resolution Plates, Type 1A. The plates were developed in Kodak D-19 followed by a stop bath and a fixing bath.

The separation between the object plane (i.e., the plane containing the random array) and the recording plane must be carefully chosen when the optical Fourier transform of the hologram is of interest. It should be recalled that a zone lens appears in the transform plane, and the frequency of the zone lens is directly proportional to the object-to-hologram separation. As noted earlier, it is necessary to remove the zone lens in effect by increasing its frequency beyond the resolution limit of the TV camera; in carrying out the experiments, it was found that a recording distance of $36 \mathrm{~cm}$ was adequate in this respect. (Since the opaque discs that comprise the random array are approximately $40 \mu \mathrm{m}$ in diameter, the $36 \mathrm{~cm}$ separation corresponds to a far-field number, $N$, of roughly 120 where $N=\lambda z / d^{2}$ )

Holograms of several random arrays consisting of $40 \mu$ discs were recorded; each array contained 5000 disc-pairs uniformly distributed over a circular region $3.5 \mathrm{~cm}$ in diameter, corresponding to 10 discs per $\mathrm{mm}^{2}$. That is, $3.1 \%$ of the $3.5 \mathrm{~cm}$-wide region is covered by the opaque discs. While the exact number of particles (or discs) is not critical, there are two factors which establish a lower limit on the number of particles required. First, there must be a sufficient number of particles present so that the distribution 
of displacements can be measured using the technique described in Chapter 3 (see Section 3.6). Secondly, the intensity pattern in the transform plane of the hologram contains a strong zero-frequency component of light which tends to scatter throughout a significant portion of the transform plane. It is therefore necessary that the sample contains a sufficient number of particles per unit area, since the relative intensity of the particle-dependent component of light with respect to the zero-frequency component is dependent on the number of particles per unit area (for a given particle size). It has been observed from experiments carried out herein that 5000 disc-pairs are more than adequate to produce a diffraction pattern of sufficient intensity when using the array-size and dot-size given above.

Experimental results obtained by applying far-field holograms of the random arrays as input to the hybrid system are presented in Figs. 4.2 - 4.14. The data in each case consist of a sequence of plots beginning with the measured intensity profile in the transform plane of a far-field hologram and ending with a plot of the computed density function. In particular, the sequence consisting of Figs. 4.2 - 4.5 contains all of the intermediate results (low-pass filtering, envelope removal, etc.) and will be used to provide a detailed illustration of the data-processing technique.

Figure $4.2 \mathrm{a}$ shows the intensity profile in the transform plane of a far-field hologram of a random array of opaque discs. In this example, the separation between disc-pairs is characterized by a 

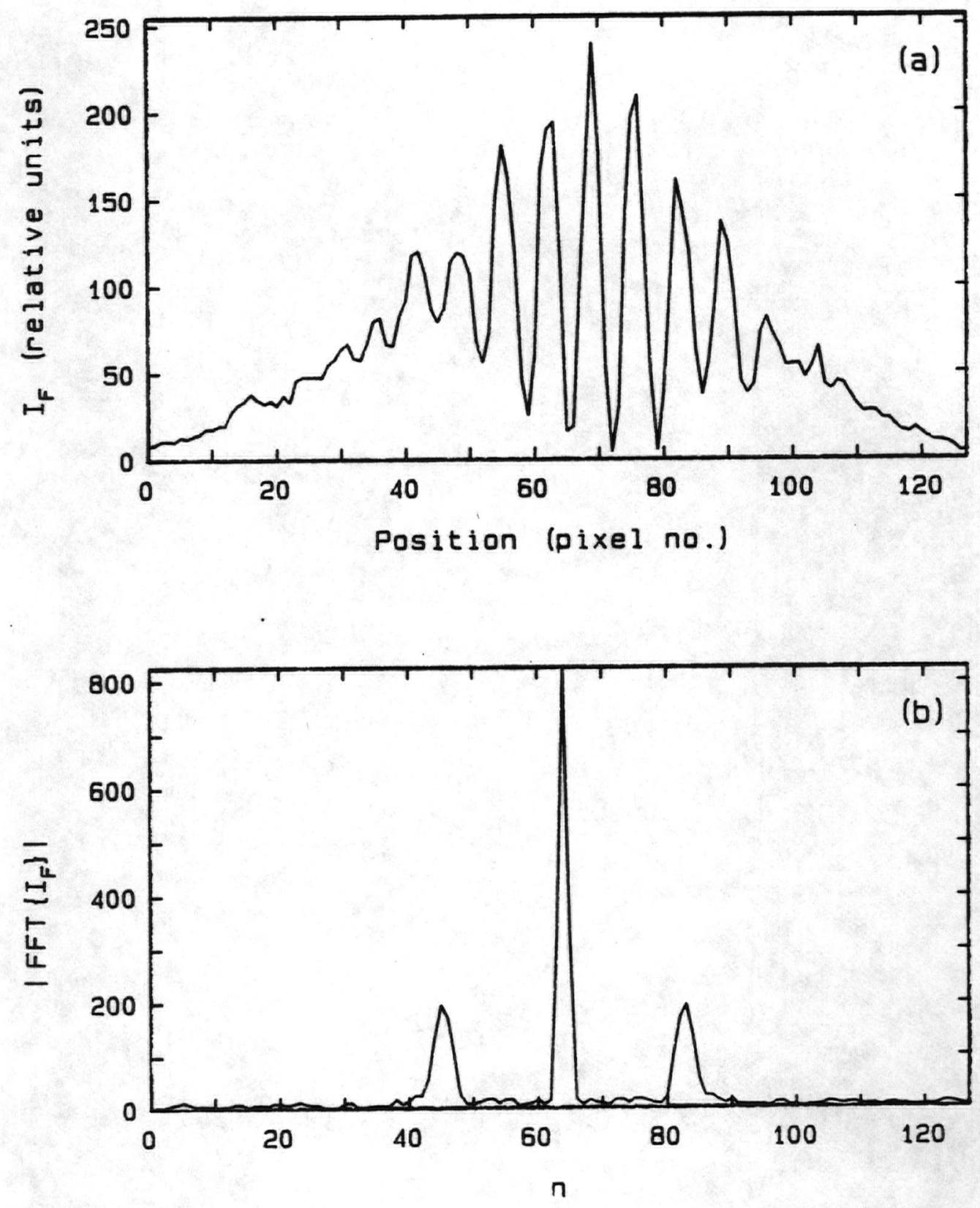

Fig. 4.2. (a) Plot of the intensity profile in the transform plane of a far-field hologram of a random array of opaque discs. The separation between disc-pairs is characterized by a Gaussian probability density function (pdf); $\mu=460 \mu \mathrm{m}$ and $\sigma=30 \mu \mathrm{m}$. (b) Magnitude of the FFT of the intensity profile vs. the discrete transform variable $n$. 

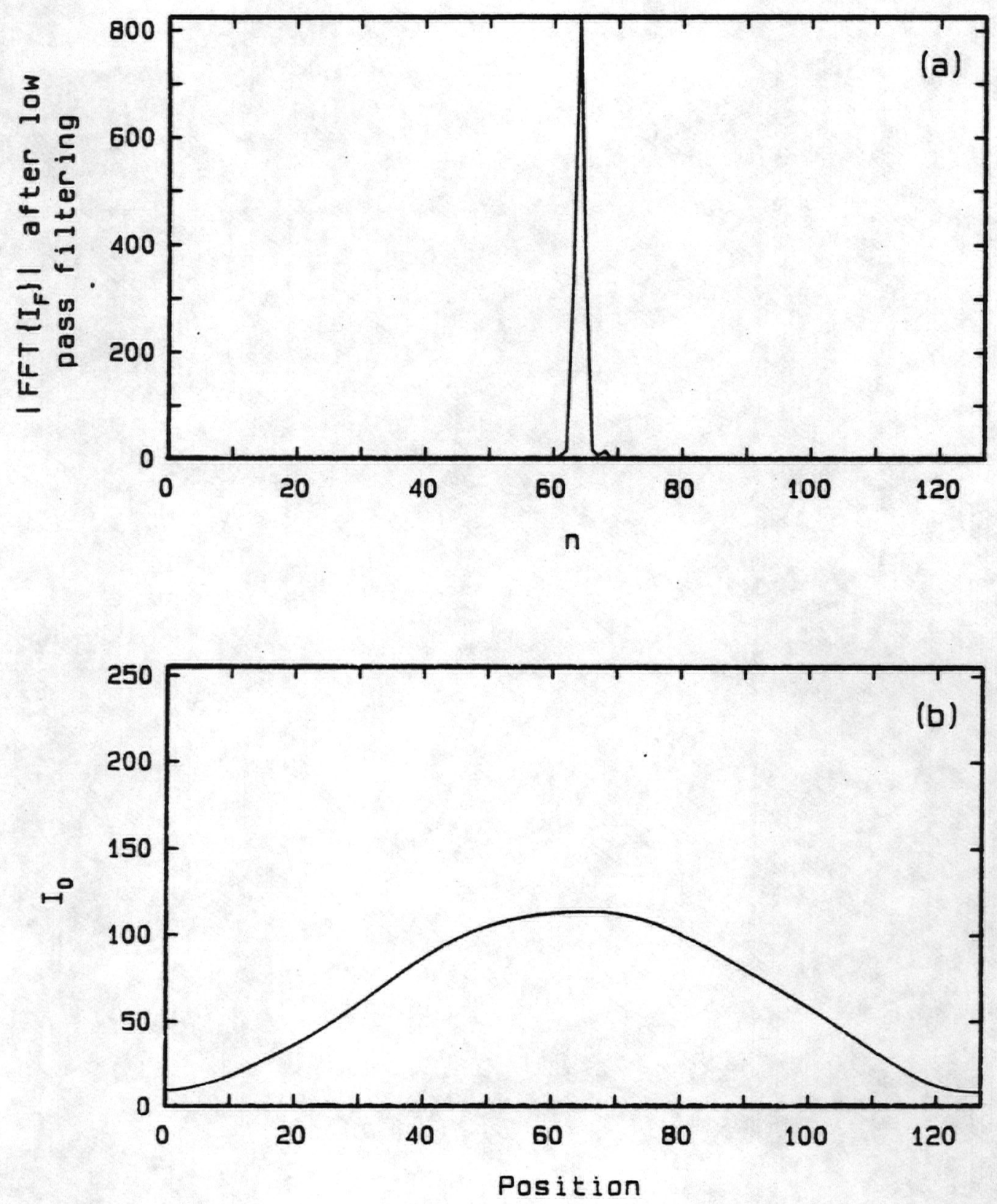

Fig. 4.3. (a) Plot of $\mid$ FFT $\left\{I_{F}\right\} \mid$ vs. $n$ after low pass filtering. (b) The envelope function $I_{0}$ is obtained by computing the inverse FFT of the data in (a). 

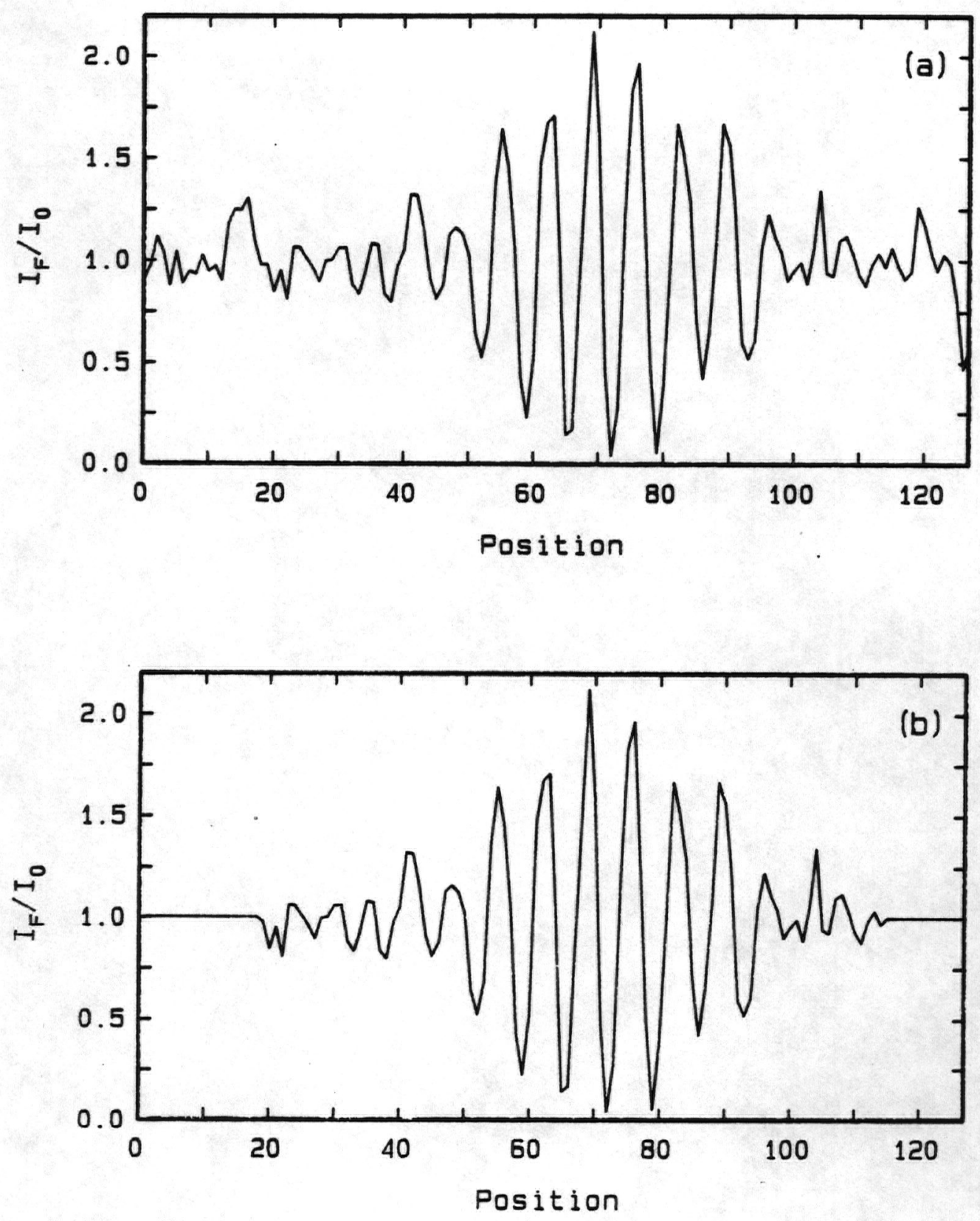

Fig. 4.4. (a) Plot of $I_{F} / I_{0}$ vs. position. (b) Same as (a) except the random fluctuations at the left and right portions of the curve were removed by setting $I_{F} / I_{0}$ to unity. 

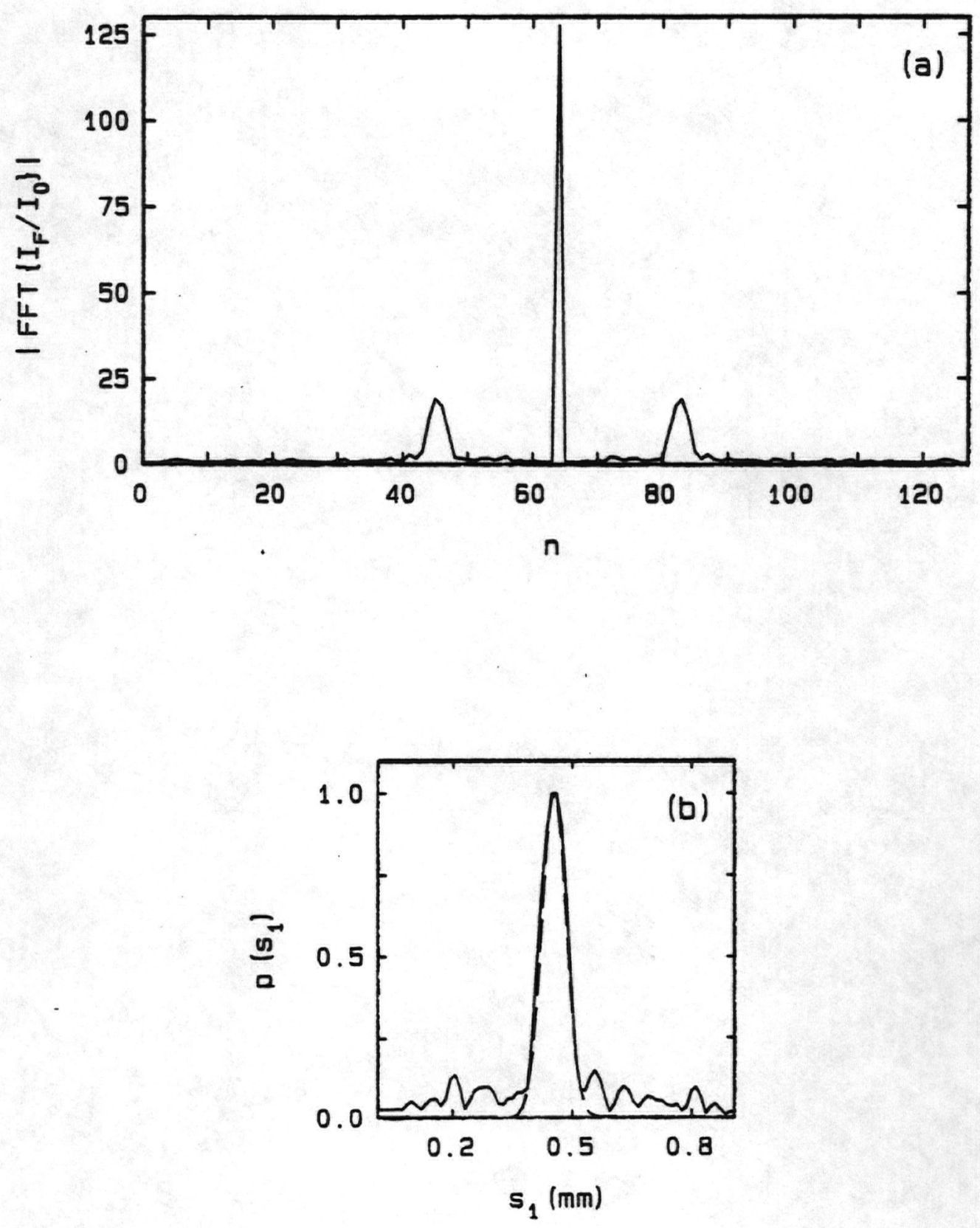

Fig. 4.5. (a) Magnitude of FFT $\left\{I_{F} / I_{0}\right\}$ vs. the discrete transform variable $n$. (b) Plot of the computed ( - ) density function and the theoretical (--) density function vs. displacement. 

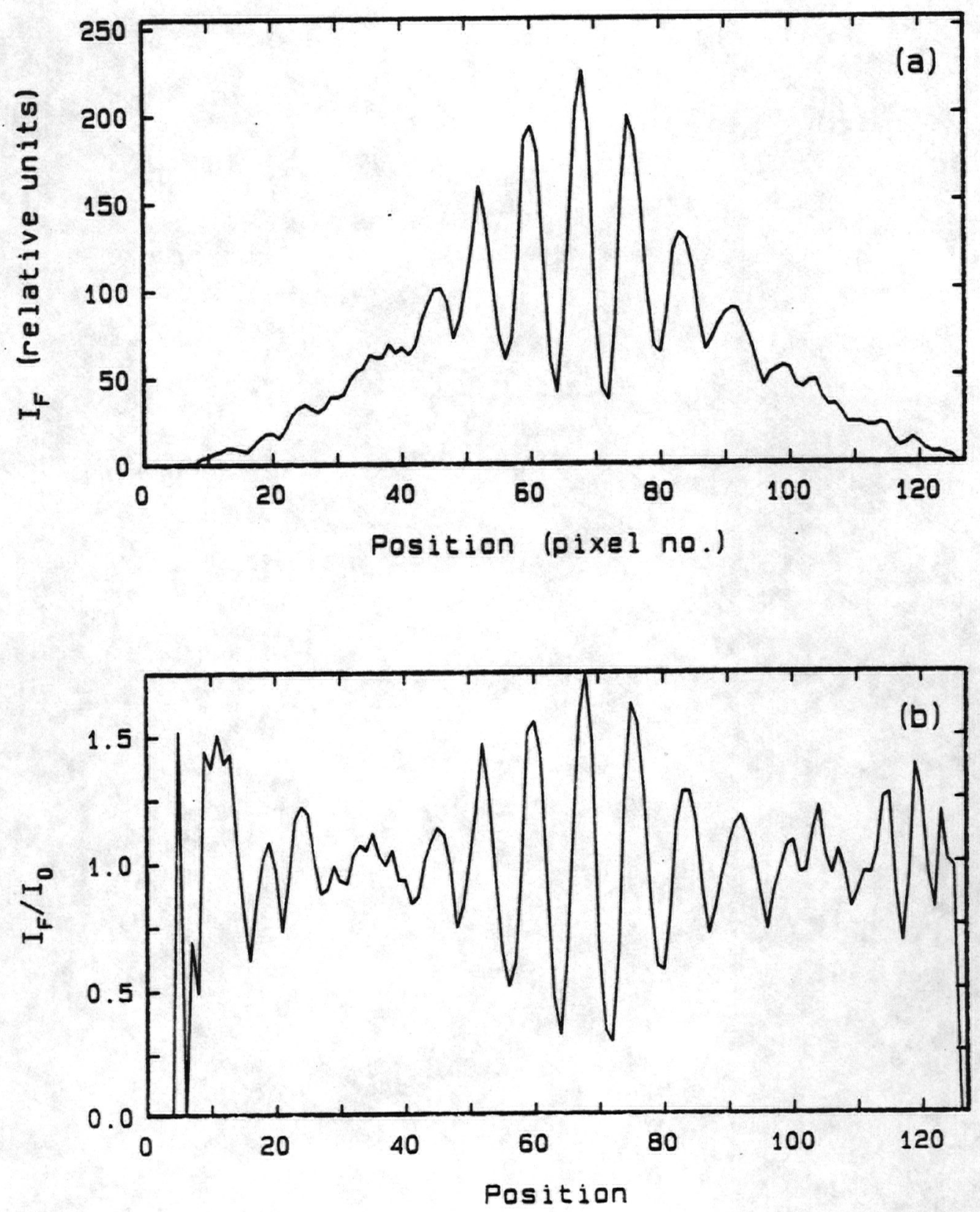

Fig. 4.6. (a) Plot of the intensity profile in the transform plane of a far-field hologram of a random array of opaque discs. The separation between disc-pairs is characterized by a Gaussian pdf; $\mu$ $=420 \mu \mathrm{m}$ and $\sigma=40 \mu \mathrm{m}$. (b) Plot of the intensity profile, $I_{F}$, divided by the envelope $I_{0}$. 

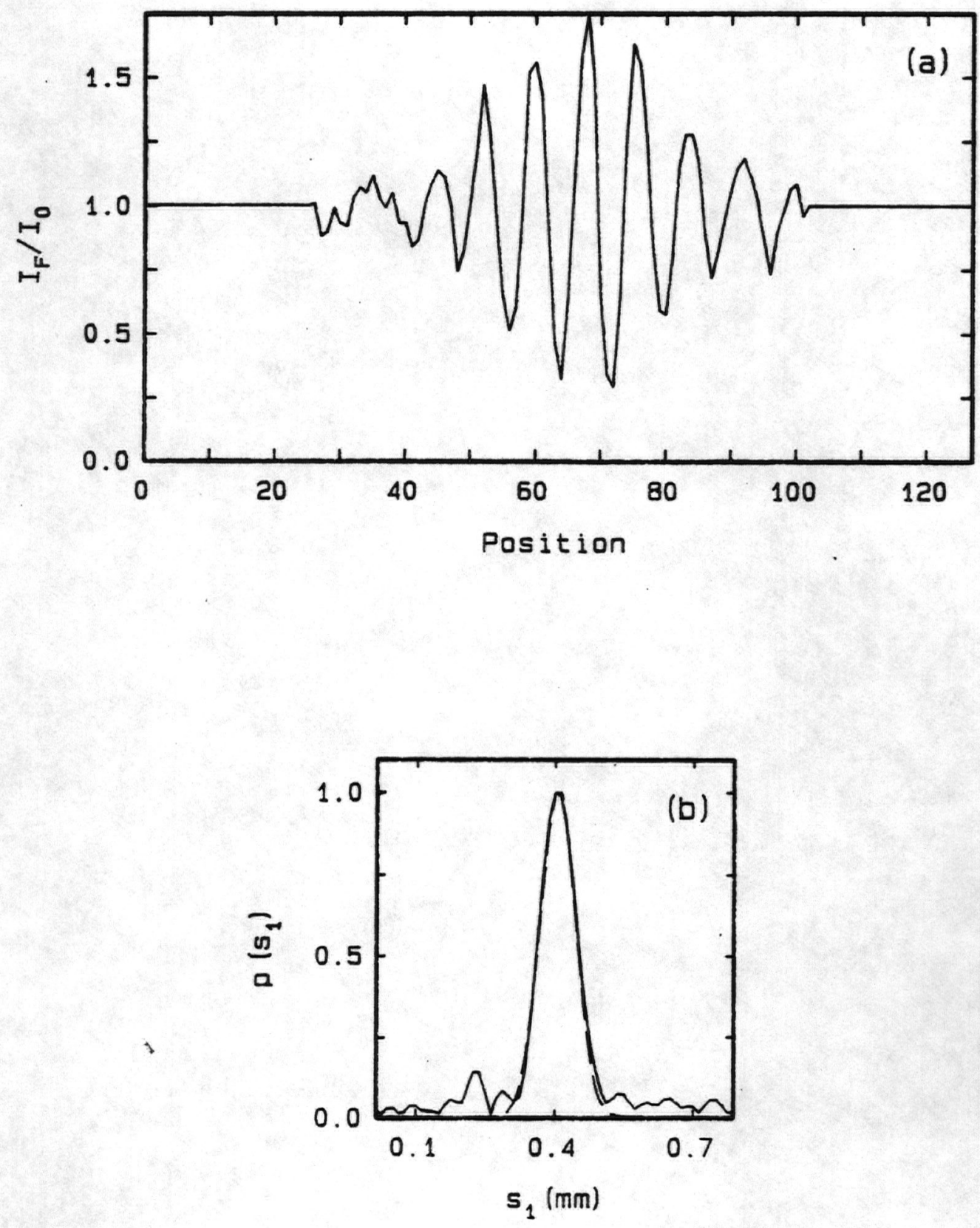

Fig. 4.7. (a) Same as $4.6 \mathrm{~b}$ except the random fluctuations at the left and right portions of the curve were removed. (b) Plot of the computed $(-)$ density function and the theoretical (--) density function vs. displacement. 

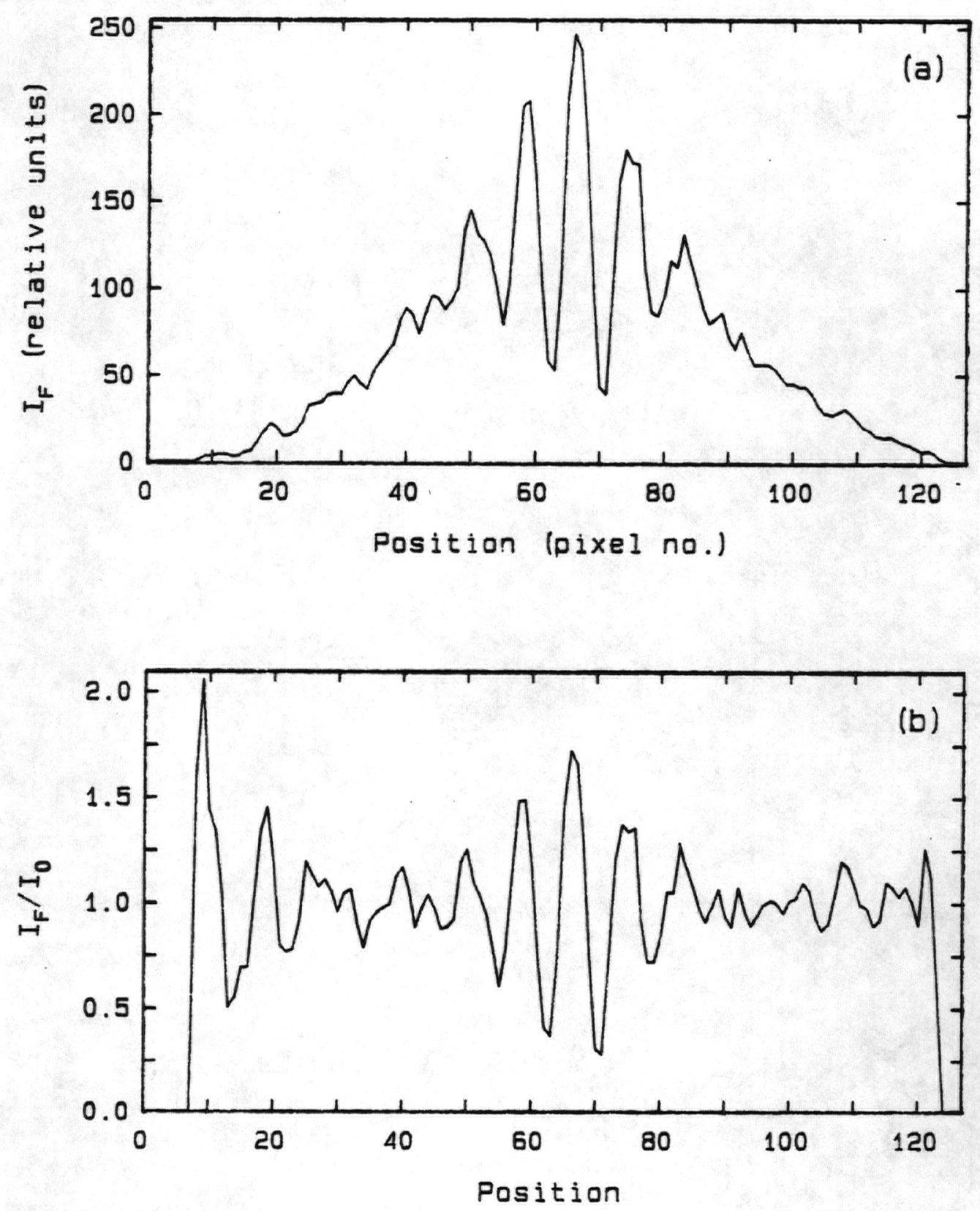

Fig. 4.8. (a) Plot of the intensity profile in the transform plane of a far-field hologram of a random array of opaque discs. The separation between disc-pairs is characterized by a Gaussian pdf; $\mu$ $=400 \mu \mathrm{m}$ and $\sigma=50 \mu \mathrm{m}$. (b) Plot of the intensity profile, $I_{F}$, divided by the envelope $I_{0}$. 

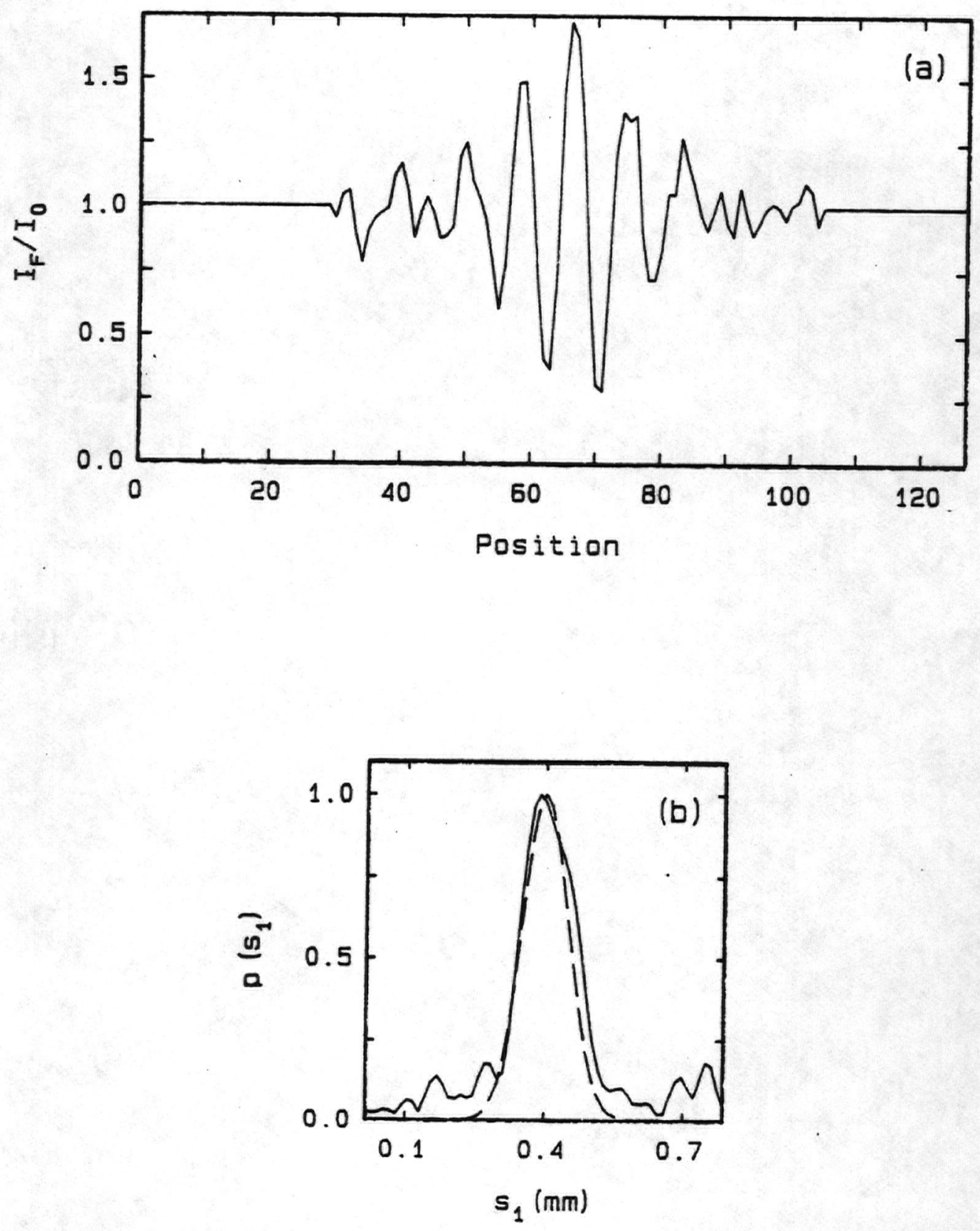

Fig. 4.9. (a) Same as $4.8 \mathrm{~b}$ except the random fluctuations at the left and right portions of the curve were removed. (b) Plot of the computed (-) density function and the theoretical (-) density function vs. displacement. 

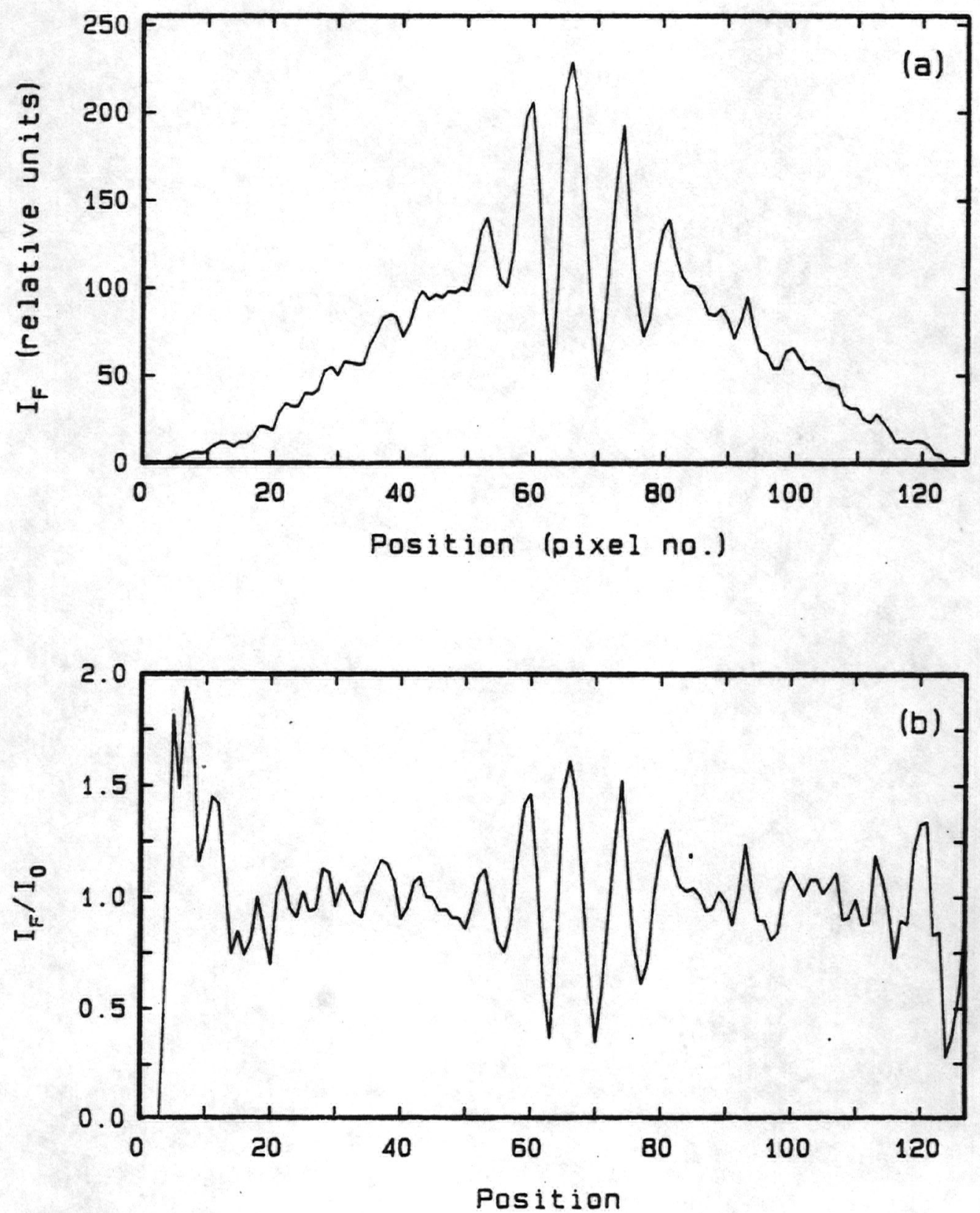

Fig. 4.10. (a) Plot of the intensity profile in the transform plane of a far-field hologram of a random array of opaque discs. The separation between disc-pairs is characterized by a Gaussian pdf; $\mu$ $=460 \mu \mathrm{m}$ and $\sigma=60 \mu \mathrm{m}$. (b) Plot of the intensity profile, $I_{F}$, divided by the envelope $I_{0}$. 

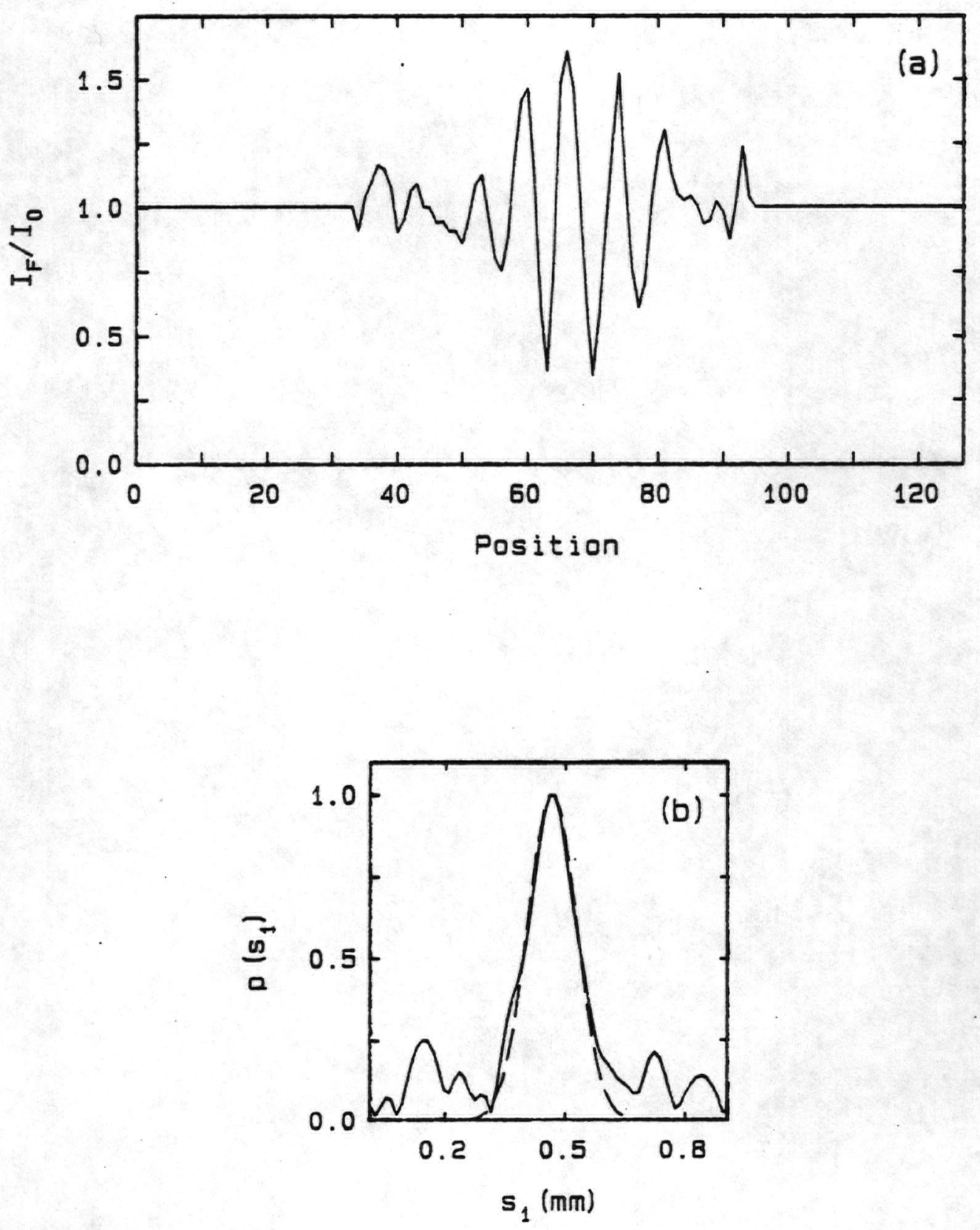

Fig. 4.11. (a) Same as $4.10 \mathrm{~b}$ except the random fluctuations at the left and right portions of the curve were removed. (b) Plot of the computed ( $(-)$ density function and the theoretical (--) density function vs. displacement. 

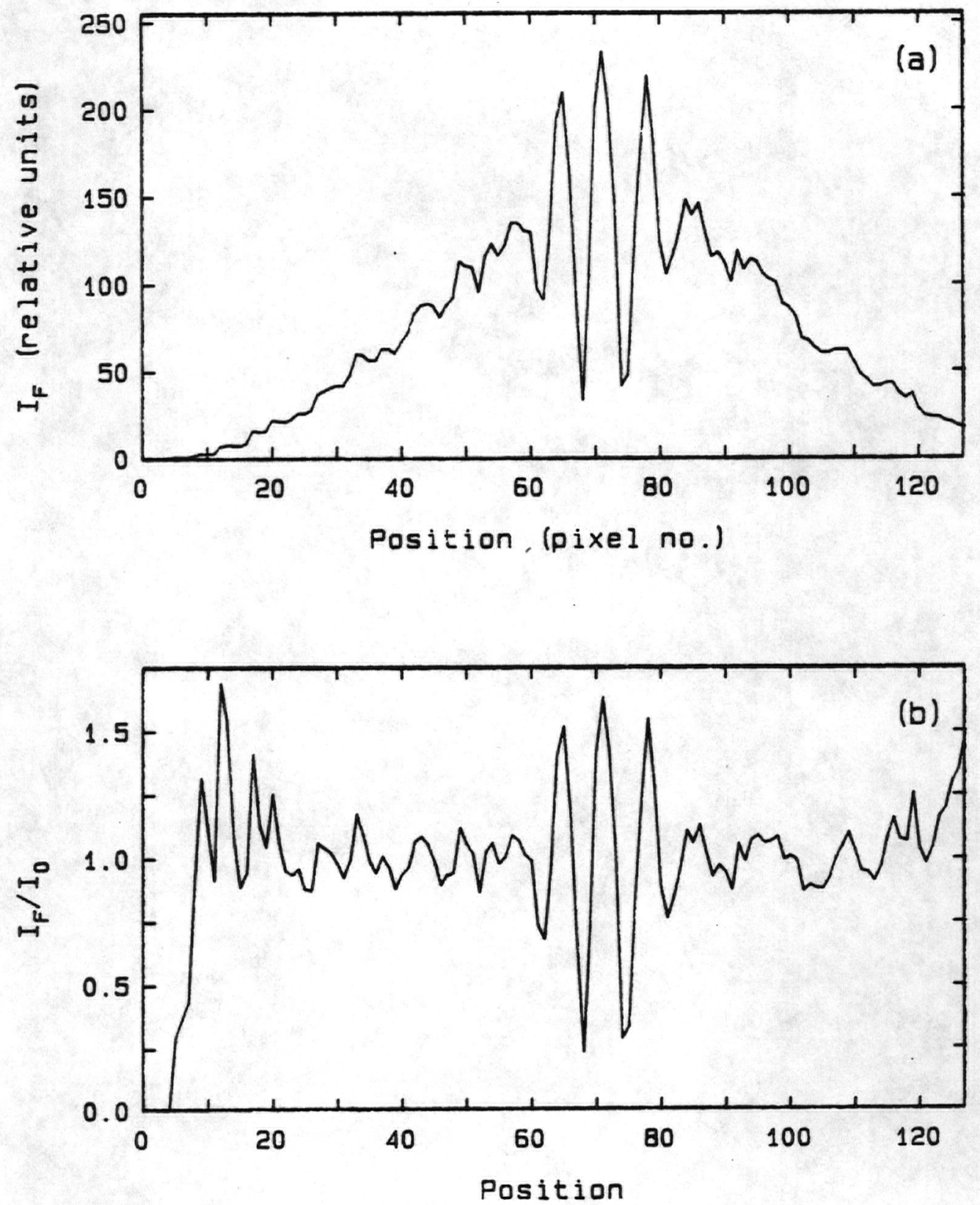

Fig. 4.12. (a) Plot of the intensity profile in the transform plane of a far-field hologram of a random array of opaque discs. The separation between disc-pairs is characterized by a Gaussian pdf; $\mu$ $=490 \mu \mathrm{m}$ and $\sigma=70 \mu \mathrm{m}$. ( (b) Plot of the intensity profile, $I_{F}$, divided by the envleope $I_{0}$. 

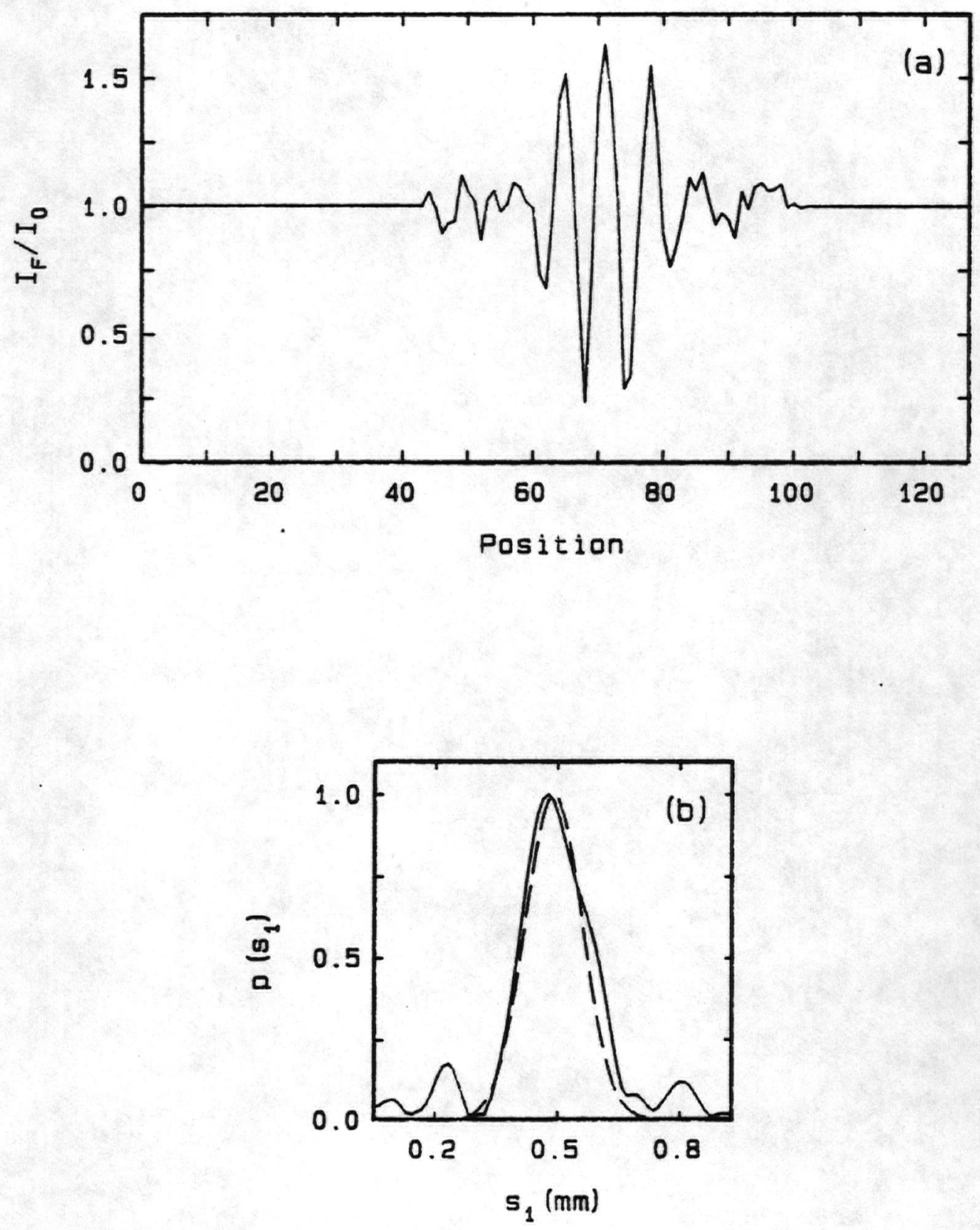

Fig. 4.13. (a) Same as $4.12 \mathrm{~b}$ except the random fluctuations at the left and right portions of the curve were removed. (b) Plot of the computed ( $(-)$ density function and the theoretical (--) density function vs. displacement. 

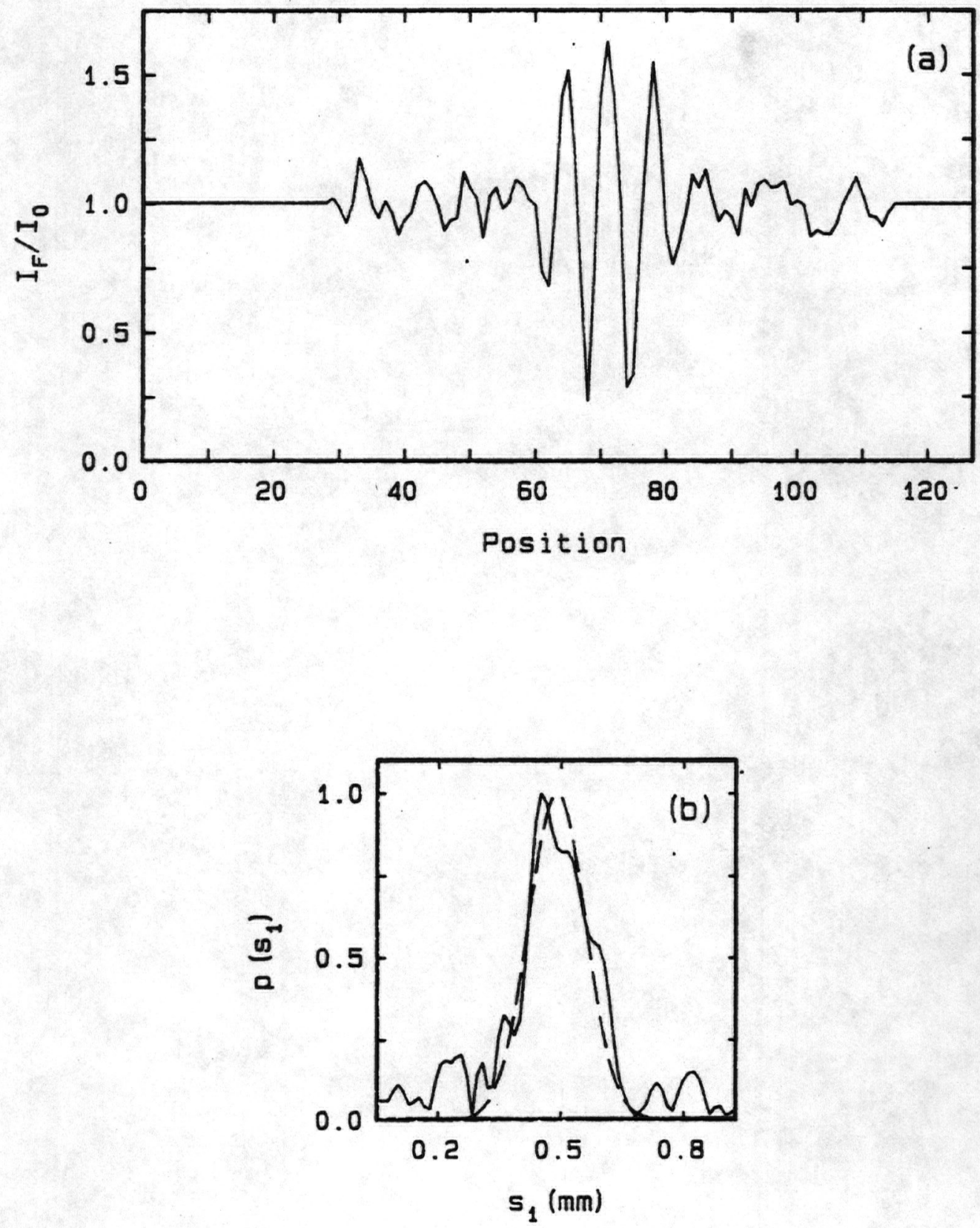

Fig. 4.14. (a) Same as 4.12 b except a relatively small portion of the random fluctuations at the left and right extremes of the curve were removed. (b) Plot of the computed (-) density function and the theoretical (--) density function vs. displacement. 
Gaussian density function having a mean of $460 \mu \mathrm{m}$ and a standard deviation of $30 \mu \mathrm{m}$. The intensity is plotted on a relative scale of 0 to 255 , corresponding to the 256 gray-levels provided by the image digitizer. Furthermore, the intensity pattern was sampled at 128 intervals along a direction perpendicular to the fringe pattern. Thus, the position along the horizontal axis in Fig. 4.2a is expressed in terms of integers ranging from 0 to 127 where each integer corresponds to a particular pixel; the actual width of the portion of the diffraction pattern depicted in Fig. 42.a is approximately $14 \mathrm{~mm}$. It should also be mentioned that 10 realizations of the measured intensity profile were averaged to reduce random noise introduced by the detector and associated electronics. (Each realization represents the measured intensity profile obtained from a single frame of video data.) In addition, 4 adjacent lines of video data were averaged to reduce fluctuations due to speckle noise.

It should be recalled from the previous chapter that once the intensity profile is obtained, a digital low-pass filter is applied to this data to determine the particle-size-dependent envelope. The filtering process is illustrated in Figs. 4.2b - 4.3b: Fig. 4.2b shows the magnitude of the FFT of the intensity profile vs. the discrete transform variable $n$, Fig. $4.3 \mathrm{a}$ shows the magnitude of the FFT after low-pass filtering and finally, the inverse transform is computed to obtain the envelope function (Fig. 4.3b). 
The results of the remaining steps in the data processing algorithm are shown in Figs. 4.4 - 4.5. Dividing the measured intensity profile, $I_{F}$, by the envelope function, $I_{0}$, yields the curve shown in Fig. 4.4a. As discussed in Section 3.5, it is necessary to remove the random fluctuations at the left and right extremes of this curve; therefore, $I_{F} / I_{0}$ is set to unity over the intervals shown in Fig. 4.4b. Finally, the FFT of the truncated version of $I_{F} / I_{0}$ is evaluated to obtain the required density function (Fig. 4.5a). The portion of the curve in Fig. $4.5 \mathrm{a}$ that represents the density function is redrawn in Fig. $4.5 \mathrm{~b}$ where the peak value of this curve is normalized to unity for convenience. To compare the shape of the experimentally obtained curve with the theoretical curve, the theoretical Gaussian density function was plotted so that its peak value coincides with that of the curve obtained experimentally (see Fig. 4.5b). Upon comparing the two curves, it is observed that there is excellent agreement except for a relatively small region near each of the tails of the Gaussian. In this region, the amplitude of the Gaussian is low with respect to the mean noise level, hence, the tails are not observed.

The results shown in Figs. 4.6 - 4.14 were obtained by applying far-field holograms of various random arrays as input to the hybrid system and processing the data as outlined above. In each case, the separation between the disc-pairs comprising the array was determined by a Gaussian random number generator. 
However, the standard deviation, $\sigma$, was increased in each successive example to demonstrate the ability to measure wider distributions.

Figures $4.6-4.7$ show the results of a Gaussian distribution with $\sigma=40 \mu \mathrm{m}$ and $\mu=420 \mu \mathrm{m}$. The intensity profile in the transform plane of the hologram is given in Fig. $4.6 \mathrm{a}$ and the result of dividing the intensity profile, $I_{F}$, by the envelope function, $I_{0}$, is given in Fig. 4.6b. (Since the filtering operation used to obtain the envelope function is so similar from example to example, it is not shown here or in the examples to follow.) The result of truncating the noisy portion of the $I_{F} / I_{0}$-curve is shown in $4.7 \mathrm{a}$ and the computed density function is plotted in Fig. $4.7 \mathrm{~b}$ along with the theoretical curve. Once again, the agreement between the two curves is quite good. In addition, similar results are given as follows: Figs. 4.8 - 4.9 pertain to a Gaussian distribution with $\sigma=50 \mu \mathrm{m}$ and $\mu=400 \mu \mathrm{m}$; Figs. $4.10-4.11$ pertain to a Gaussian distribution with $\sigma=60 \mu \mathrm{m}$ and $\mu=460 \mu \mathrm{m}$ and Figs. $4.12-4.13$ pertain to a Gaussian distribution with $\sigma=70 \mu \mathrm{m}$ and $\mu=490 \mu \mathrm{m}$.

It is observed from Figs. 4.2 - 4.12 that there exists a certain interval in the optical transform plane over which displacement-dependent fringes are visible; as $\sigma$ increases, the width of this interval steadily decreases. (Clearly, this result is expected from the theoretical model developed in Chapter 3.) Outside of this interval, random fluctuations primarily due to speckle noise are observed (see, for example, Fig. 4.12b). As demonstrated in Figs. 4.13 and 4.14 , it is necessary to remove the 
fluctuations which lie outside the aforementioned interval to accurately obtain the desired density function. In Fig. 4.13a, a significant portion of the fluctuations were removed and, as a result, the computed density function is in good agreement with the theoretical density function (see Fig. 4.13b). However, in Fig. 4.14a, a relatively small portion of the random fluctuations were removed, and the computed density function that results (Fig. 4.14b) does not agree very well with the theoretical density function. Nevertheless, if semi-automatic data processing is used, the operator may easily determine when the fringe visibility in the transform plane approaches zero by visually inspecting the transform pattern on the video monitor. As a result, the appropriate intervals for truncating the random fluctuations may readily be determined and the required density function may be accurately obtained.

The final set of data presented in this subsection (Figs.4.15 - 4.16) was derived from a far-field hologram of a random array of discs in which the separation between disc-pairs was determined by a uniform random number generator. Specifically, the separation between disc pairs is uniformly distributed between $385 \mu \mathrm{m}$ and 535 $\mu \mathrm{m}$. The intensity profile in the transform plane of this hologram is shown in Fig. 4.15a. Obtaining the envelope function in the manner indicated earlier and dividing the intensity profile by the envelope yields the curve shown in Fig. 4.15b. Truncating the random fluctuations near the left and right portions of the curve 

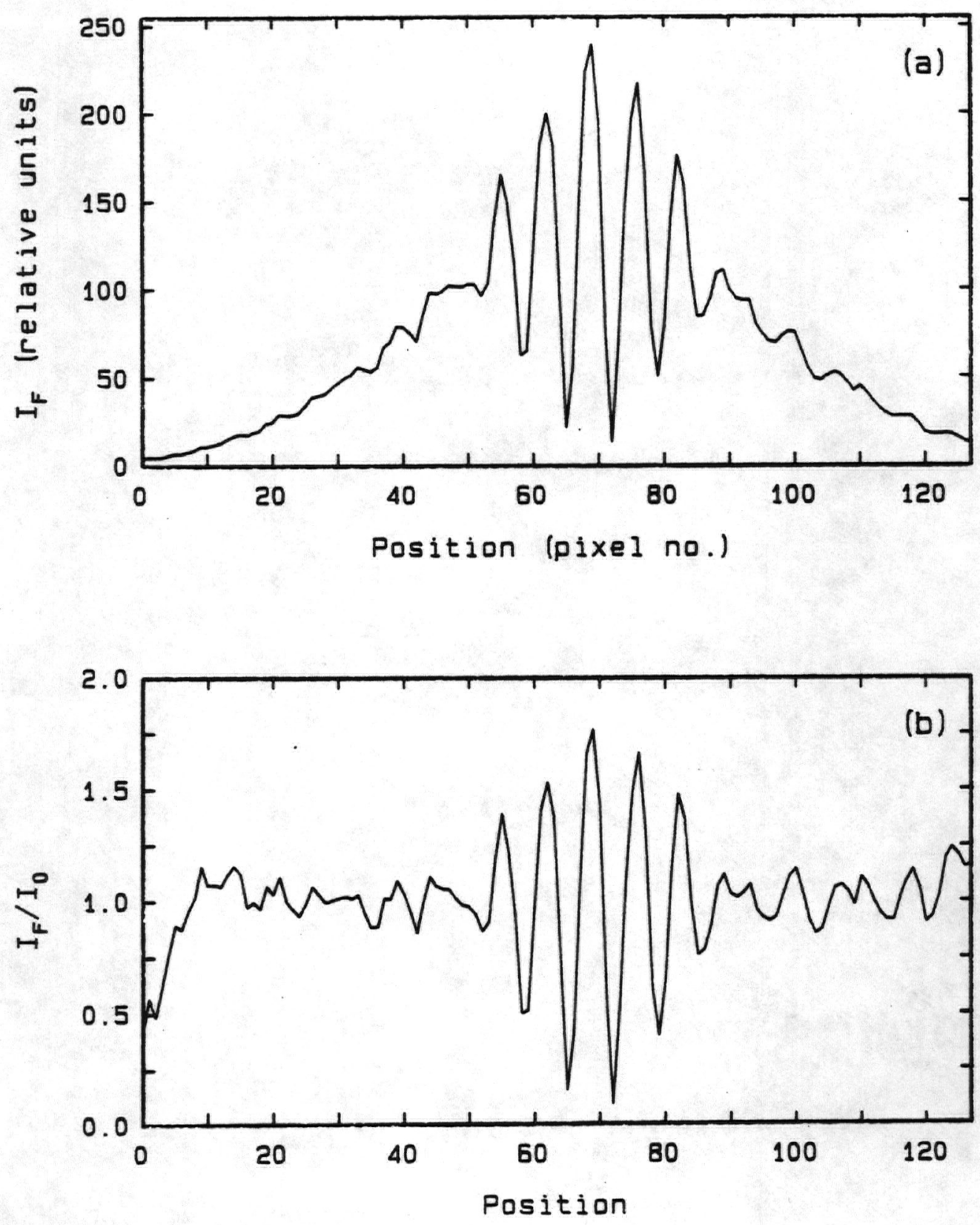

Fig. 4.15. (a) Plot of the intensity profile in the transform plane of a far-field hologram of a random array of opaque discs. The separation between disc-pairs is uniformly distributed between $385 \mu \mathrm{m}$ and $535 \mu \mathrm{m}$. (b) Plot of the intensity profile, $I_{F}$, divided by the envelope $I_{0}$. 

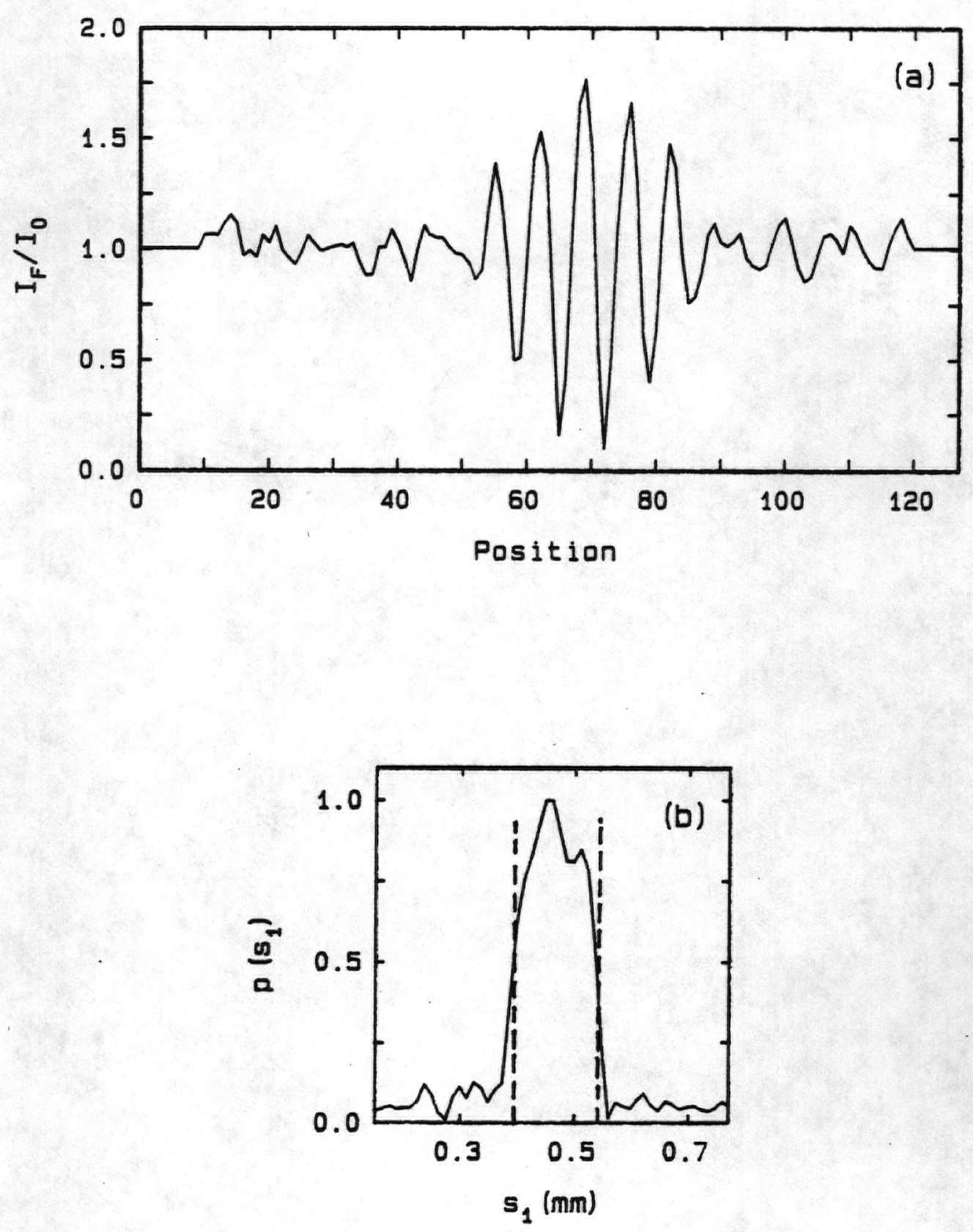

Fig. 4.16. (a) Same as $4.15 \mathrm{~b}$ except the random fluctuations at the left and right portions of the curve were removed. (b) Plot of the computed ( $(-)$ density function and the theoretical (--) density function vs. displacement. 
in Fig. 4.15b gives the result shown in Fig. 4.16a. Finally, the computed density function together with the theoretical density function is shown in Fig. 4.16b. The computed density function is in reasonably close agreement with the theoretical curve although not as close as the corresponding curves obtained in the case of a Gaussian distribution (see Figs. 4.2 - 4.14). This result is expected and may be explained by considering the theoretical expressions that describe the intensity pattern in the optical transform plane for each of the respective distributions. It should be recalled from the previous chapter (Section 3.3.3) that the intensity in the transform plane of a double-exposure hologram for which the particles have a Gaussian distribution displacements is given by:

$$
<I_{F}(u, v)>=I_{0}(u, v)\left\{1+\exp \left[-\frac{2 \pi^{2} \sigma^{2} u^{2}}{\lambda^{2} f^{2}}\right] \cos \left(\frac{2 \pi \mu u}{\lambda f}\right)\right\}
$$

For a uniform distribution of displacements (see Section 3.3.2), the corresponding expression is

$$
<I_{F}(u, v)>=I_{0}(u, v)\left\{1+\operatorname{sinc}\left(\frac{2 u}{\lambda f} \frac{(c-b)}{2}\right) \cos \left(\frac{2 \pi \mu}{\lambda f} \frac{(c+b)}{2}\right)\right\},
$$

where $b$ and $c$ denote the minimum and maximum particle displacements, respectively. Thus, the visibility of the fringes falls off as $\exp \left(-u^{2}\right)$ for the Gaussian distribution whereas the visibility falls off as $1 / u$ for the uniform distribution. Clearly, the negative 
exponential decays much more rapidly than $1 / u$, and hence, the fringe pattern observed in the case of the Gaussian distribution is effectively confined to a relatively narrow interval in the transform plane when compared to the fringe pattern observed in the case of the uniform distribution (for a given distribution width). Since $1 / u$ gradually approaches zero, a large number of low contrast fringes must be detected to compute the required density function. However, the low contrast fringes are difficult to detect, primarily because they generally occur in the region of the optical transform plane where the envelope function, $I_{0}(u, v)$, tends to 0 . In addition, the data are further degraded by the random fluctuations in the intensity pattern due to speckle. As a result, it is difficult to accurately determine the fringe pattern over the interval required, consequently, it is difficult to compute the required density function with high accuracy. In practice, however, actual particles in motion are more likely to acquire a Gaussian or a Gaussian-like distribution rather than a uniform distribution of velocity.

\subsubsection{Measuring the velocity distribution of particles falling in water}

An example to demonstrate that the hybrid system for automated readout may be applied in practice is presented in this subsection. In particular, a velocity distribution was obtained by allowing $79 \mu \mathrm{m}$ pollen particles (corn pollen prepared by Coulter Electronics, Ltd.) to fall in water. The resulting particle motion was slow enough to allow the use of a chopped cw laser beam for recording the 
hologram. (Particle motion during exposure is addressed in Appendix C.) Specifically, a double-exposure far-field hologram was recorded onto a Kodak High Speed Holographic Plate, Type 131-02 using an Argon-ion laser operating at $5145 \AA$. A mechanical shutter was used to produce two pulses, each having a duration of $1 \mathrm{msec}$; the separation between pulses was $33 \mathrm{msec}$. The intensity profile in the transform plane of the resulting hologram is shown in Fig. 4.17a. Processing the data in the prescribed manner yields the intermediate results shown in Figs. $4.17 \mathrm{~b}$ and $4.18 \mathrm{a}$. Finally, the density function which characterizes the velocity distribution is given in Fig. $4.18 \mathrm{~b}$ where it is observed that the distribution is Gaussianlike with a mean speed of approximately $2.5 \mathrm{~mm} / \mathrm{sec}$.

It is interesting to note that the falling particles acquire a distribution of velocities rather than acquiring a constant terminal velocity. This is apparently dependent on the separation between particles and hence the number density. When visually observing a small number of well-separated particles, the individual particles appear to reach a constant velocity soon after being released in the water. However, when many particles are allowed to fall in the liquid, the motion of any given particle is influenced by the motion of the surrounding particles since a moving particle will disturb the liquid immediately surrounding it. The net result is that the particles assume a distribution of velocities. 

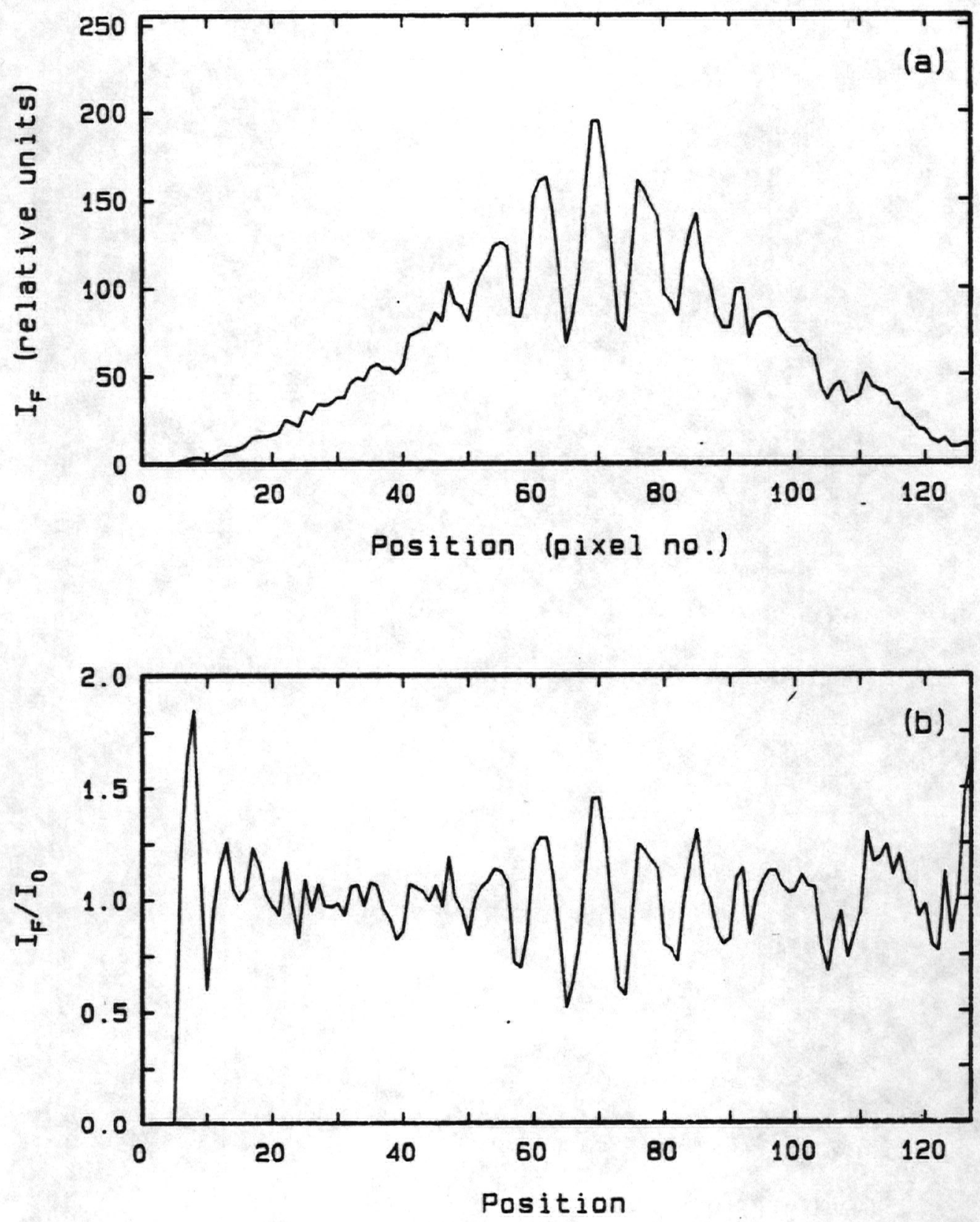

Fig. 4.17. (a) Intensity profile in the transform plane of a double-exposure far-field hologram of $79 \mu \mathrm{m}$ particles falling in water. (b) Plot of the intensity profile, $I_{F}$, divided by the envelope $I_{0}$. 

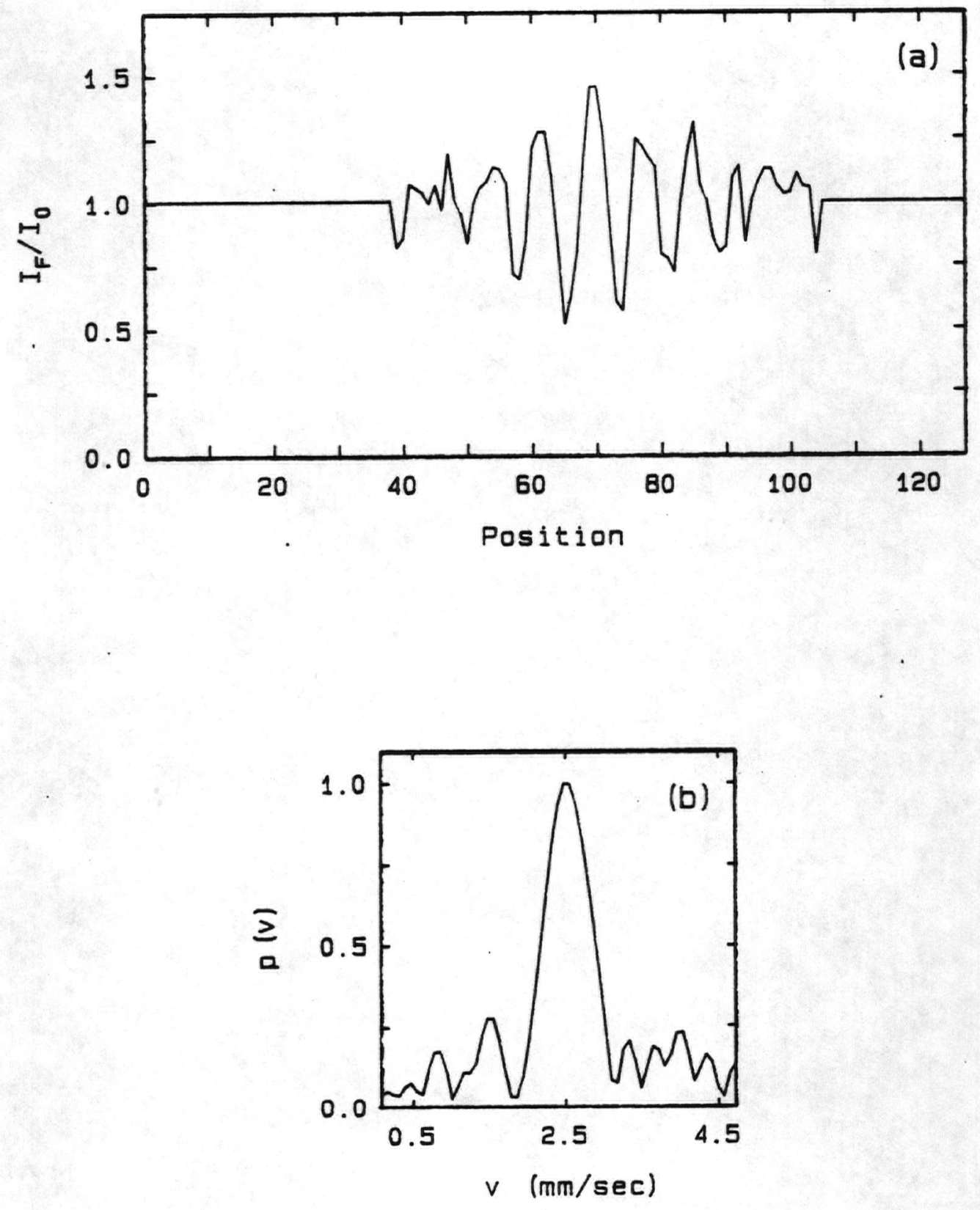

Fig. 4.18. (a) Same as 4.17 b except the random fluctuations at the left and right portions of the curve were removed. (b) Plot of the experimentally obtained density function which characterizes the velocity distribution. 


\subsection{Two-dimensional distributions: measuring the marginal density function}

It was shown in the previous chapter (Section 3.8 ) that in the case of a two-dimensional distribution of particle displacements, the marginal density functions may be obtained if the horizontal and vertical components of displacement are statistically independent. It will be recalled that each of the marginal density functions may be obtained by measuring the intensity profile in the transform plane in two orthogonal directions. Namely, one scan is taken in a direction parallel to the horizontal axis and another is taken parallel to the vertical axis in the transform plane. Processing each of the arrays of data (in the same manner as data corresponding to a one-dimensional distribution) will yield the marginal density functions. If desired, the joint density function that characterizes the particle displacements in two dimensions may then be obtained by forming the product of the two marginal density functions.

To demonstrate that the marginal density function may indeed be measured, a random array of opaque discs was once again used to simulate particles in motion. In this case, however, the displacement between the discs which comprise a typical disc-pair was random in two dimensions. That is, the horizontal and vertical components of displacement were random, and each component was determined by a Gaussian random number generator. In particular, the mean and standard deviation of the horizontal component of 
displacement is denoted by $\mu_{1}$ and $\sigma_{1}$, respectively, where $\mu_{1}=450 \mu \mathrm{m}$ and $\sigma_{1}=35 \mu \mathrm{m}$. Similarly, the parameters for the vertical component are given by $\mu_{2}$ and $\sigma_{2}$ where $\mu_{2}=\mu_{1}=450 \mu \mathrm{m}$ and $\sigma_{2}=\sigma_{1}=35 \mu \mathrm{m}$. The resulting intensity profile in the transform plane of a hologram of the random array is shown in Fig. 4.19a. As expected, the fringe visibility is considerably lower than that exhibited by a onedimensional distribution ( $c$. Fig. 4.2a). Processing the data in the prescribed manner yields the results shown in Figs. 4.19b - 4.20. The intensity profile with the envelope function removed is shown in Fig.4.19b. Removing the random fluctuations at the left and right portions of the curve in Fig. $4.19 \mathrm{~b}$ yields the curve in Fig. $4.20 \mathrm{a}$, and finally, the measured density function together with the theoretical density function is shown in Fig. 4.20b. The agreement between theory and experiment is very good. However, the mean noise level with respect is considerably higher than that of a onedimensional distribution of comparable width (see Fig. 4.5b) as a result of the relatively low-contrast fringes in the transform plane.

\subsection{Limitations on the width of the velocity distribution}

The results presented in the previous sections clearly indicate that double-exposure holography coupled with the hybrid readout system can be a useful tool for measuring particle velocity distributions. There are, however, limitations to this technique 

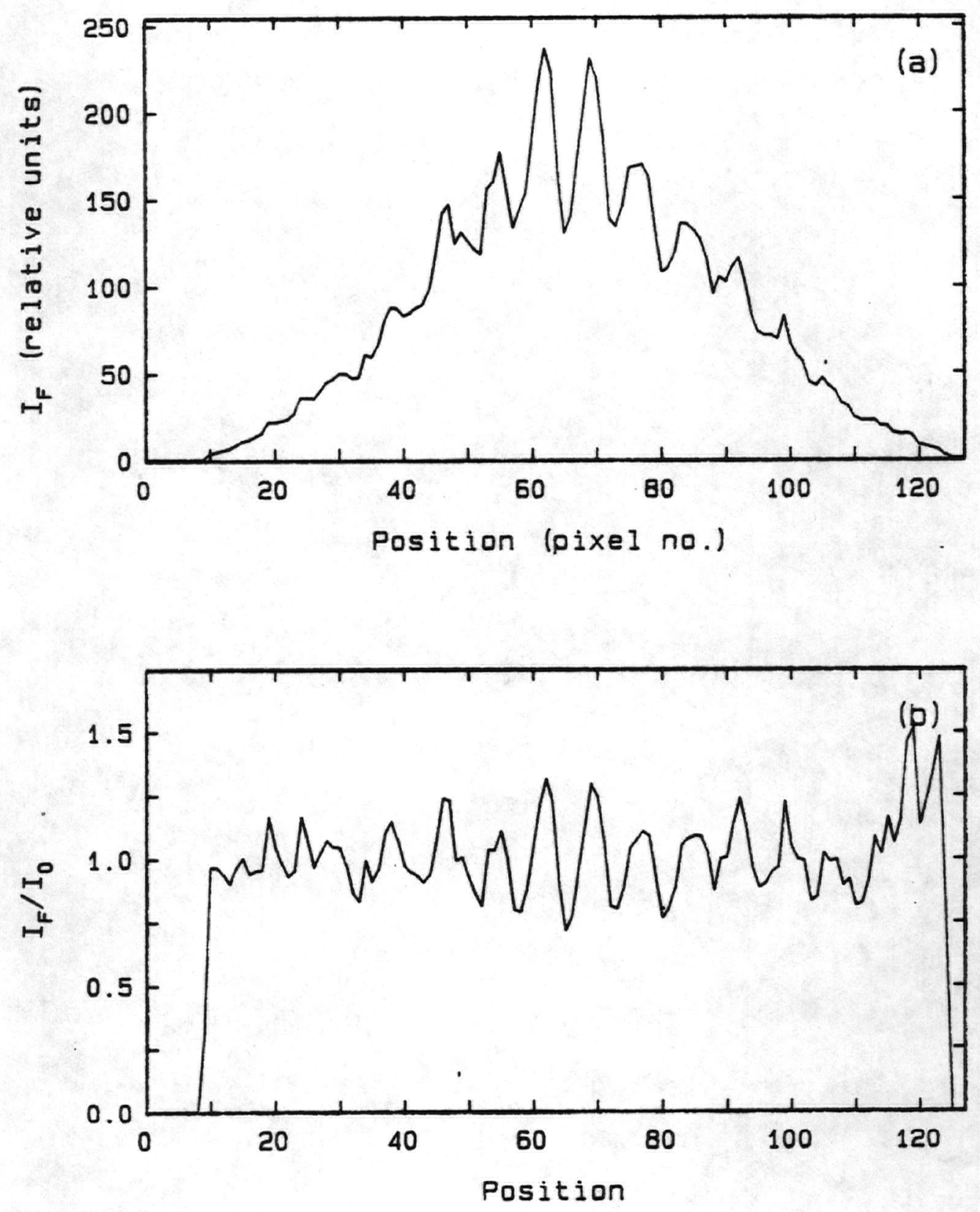

Fig. 4.19. (a) Plot of the intensity profile in the transform plane of a far-field hologram of a random array of opaque discs. The separation between disc pairs is Gaussian in two-dimensions; $\mu_{1}=\mu_{2}=$ $450 \mu \mathrm{m}$ and $\sigma_{1}=\sigma_{2}=35 \mu \mathrm{m}$. (b) Plot of the intensity profile, $I_{F}$, divided by the envelope, $I_{0}$. 

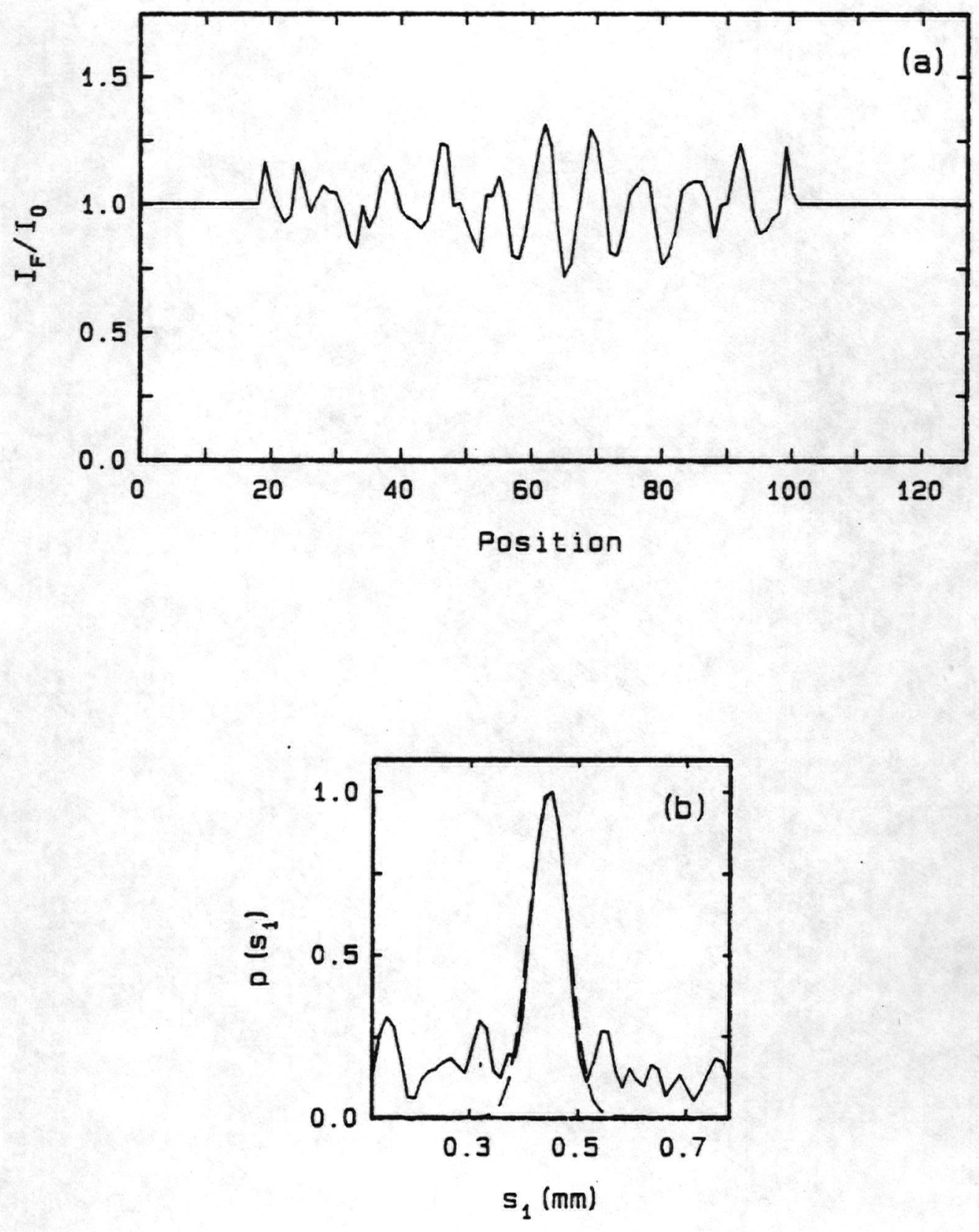

Fig. 4.20. (a) Same as $4.19 \mathrm{~b}$ except the random fluctuations at the left and right portions of the curve were removed. (b) Plot of the computed ( $(-)$ marginal density function and the theoretical (-) density function vs. displacement. 
since a distribution which is either too wide or too narrow cannot be accurately measured. (See also Section 3.3.5.)

The narrowest distribution which may be measured is determined by the size of the particles contained in the sample volume. To illustrate, the particles are assumed to be spherical in shape and the distribution at displacements is assumed to be Gaussian. In this case, the intensity in the transform plane is given by Equation (3.31) which is rewritten below:

$$
<I_{F}(u, v)>=\pi a^{2}\left[\frac{2 J_{1}\left(\frac{2 \pi a \rho}{\lambda f}\right)}{\frac{2 \pi a \rho}{\lambda f}}\right]\left\{1+\exp \left[-\frac{2 \pi^{2} \sigma^{2} u^{2}}{\lambda^{2} f^{2}}\right] \cos \left(\frac{2 \pi \mu u}{\lambda f}\right)\right\} \cdot(4 \cdot 3)
$$

where $a$ denotes the radius of a typical particle; the remaining terms are defined in Section 3.5 and the zone lens term was omitted for convenience. In practice, the intensity profile in the transform plane is sampled over an interval which extends to the first zero of the Bessel function, $\dot{J}_{1}($.$) . Since the argument of the$ Bessel function is dependent on the quantity $a$ (the particle radius), the width of the sampling interval is inversely proportional to $a$. To obtain the required density function from the measured intensity profile, the value of the exponential term in Equation (4.3) must approach zero within the sampling interval. That is, the width of the sampling interval must exceed the effective width of the exponential term, otherwise, the measured intensity profile will not contain sufficient information to permit 
an accurate calculation of the density function. Therefore, decreasing the particle diameter allows narrower and narrower distributions to be measured. However, as the particle size decreases, it becomes difficult to record a good hologram of the particle (see Dunn and Thompson [1982]). As a result, very narrow distributions cannot be accurately measured with this technique.

It is also difficult to measure very wide distributions using the hybrid system for automated readout since the exponential term in Equation ( 4.3 ) decays rapidly when the velocity distribution is wide (i.e., $\sigma$ is large). That is, the number of fringes visible in the transform plane steadily decreases as $\sigma$ increases. As the number of fringes approaches zero, the velocity distribution clearly cannot be determined from data available in the transform plane. However, the standard deviation itself is not necessarily the limiting factor; rather, it is necessary to consider the value of $\sigma$ relative to the mean, $\mu$. If $\sigma$ is large with respect to $\mu$, fringes will not be visible in the transform plane because the cosine term in Equation (4.3) oscillates slowly in comparison to the rate at which the exponential decays. The mean particle displacement, $\mu$, may be increased in effect by translating the holographic plate between exposures (Ewan [1979]), thereby increasing the number of fringes observed. Hence, adding a constant bias to the mean displacement allows the distribution to be measured from data obtained in the transform plane. Nevertheless, the mean cannot be increased without limit. As the mean increases, the spatial period 
of the fringes decreases. However, the fringe period must clearly remain larger than the average speckle size in the transform plane if meaningful data is to be obtained.

\subsection{Summary}

Experiments were carried out to demonstrate that particle displacement and velocity distributions may be determined by properly processing intensity data obtained in the optical transform plane of a double-exposure hologram. A system consisting of a TV camera and an image digitizer was used for data acquisition, and the resulting data was then digitally processed using the algorithm developed in Chapter 3.

Holograms with known "particle displacements" were recorded to evaluate the performance of the optical-digital system. These test holograms were actually holograms of computer-generated arrays of discs in which the spacing between the individual disc-pairs was determined by a random number generator. The disc-pairs, of course, simulate moving particles which are recorded at two points in time. Therefore, using such a hologram as input to the optical-digital system allows a direct comparison between the system output (i.e., the calculated probability density function that characterizes the distribution of displacements) and the known density function. A series of holograms, in which the separation between disc-pairs is described by a Gaussian random variable, was used as input to the system and very good agreement between theory and experiment was 
obtained (see Figs. 4.2 - 4.13). As discussed in Section 4.4.1, a uniform distribution is inherently more difficult to measure with the hybrid system than a Gaussian of comparable width. Fortunately, Gaussian or Gaussian-like distributions are more likely to be encountered in practice.

Applying the velocity measurement technique to study actual particles in motion was also demonstrated. Namely, a doubleexposure hologram of $79 \mu \mathrm{m}$ particles falling in water was recorded. This hologram was then used as input to the hybrid system and the velocity distribution was obtained.

Finally, particle motion in two dimensions was considered. By using a hologram of a random array of discs as input to the opticaldigital system, it was demonstrated that the marginal density functions may be obtained if the horizontal and vertical components of displacement are statistically independent. 


\section{Chapter V}

\section{Conclusions and Suggestions for Further Study}

It is clear that holography, and far-field holography in particular, is a valuable tool in the field of particle analysis. As with any method of particulate characterization, there are certain problems and limitations associated with the holographic technique. A problem frequently encountered in practice is the tediousness involved with manually analyzing the reconstructed images to obtain the required information (i.e., particle size, shape and/or displacement). For example, Bexon et al. [1976] maintain that "...at best 400 particles can be sized in 7 hours and there is evidence to suggest that the operator becomes erratic as time passes by." Automatic analysis is clearly required to realize the full potential of the holographic technique, especially when samples consisting of many particles are to be studied.

Particle velocity and displacement information is of interest in a wide variety of technological areas; hence the development of a method for automatically determining the velocity information from a double-exposure hologram was well-motivated. In many instances, automated readout may be the only feasible means of obtaining the required information, since it can be difficult for the human observer to determine which particle images are related by 
translation when examiming the reconstruction of a doubly exposed hologram. It should be recalled that the operation of the opticaldigital system for automated readout depends on the correlation between the hologram recorded during the first exposure and the hologram recorded during the second expsoure. Unlike manual readout, it is not necessary to determine which images are related by translation.

It is interesting to consider the time savings that can be achieved when using the optical-digital readout system to obtain particle velocity information. For example, the holograms of the random arrays of discs (described in Chapter 4) consisted of 5000 disc-pairs. Assuming the displacements of 400 disc-pairs can be determined manually in 7 hours implies that approximately 88 hours will be required to measure the displacements for the entire sample. Using the optical-digital system depicted in Fig. 4.1 and processing the resulting data as indicated in Chapter 4 requires roughly 30 minutes to obtain the distribution of displacements. Of course, the time required by the computer to perform the required calculations is significantly less than 30 minutes. Most of the time is spent on the following steps: a) recording the optical transform pattern on video tape, b) playing back the video tape and c) properly setting the black level and video level on the image digitizer to make full use of the 256 available gray levels. If the output signal of the TV camera is connected directly to the image digitizer, the video 
recorder may be eliminated, thus reducing the total data processing time.

It should be recalled from Chapters 3 and 4 that the probability density function that characterizes the particle displacements is determined by: 1) measuring the intensity profile (denoted by $I_{F}$ ) in the optical Fourier transform plane of a doubleexposure hologram, 2) obtaining the particle-size-dependent envelope (denoted by $I_{0}$ ) from the measured intensity data by digital filtering, 3) dividing the measured intensity profile, $I_{F}$, by the envelope, $I_{0}$, and 4 ) computing the FFT of $I_{F} / I_{0}$. In many cases, a computational savings can be realized by eliminating steps 2,3 and 4 above. Instead, the FFT of the measured intensity profile can be used directly to provide a good approximation to the actual density function. The result of using this abbreviated procedure as compared to the usual 4-step process is illustrated in Fig. 5.1. Figure 5.1a shows the density function obtained by using the 4-step process described above. In fact, this plot is identical to Fig. 4.7b which shows the system output (i.e., the computed density function) for a hologram of a random array of discs. The spacing between the disc-pairs is characterized by a Gaussian density function with $\mu=420 \mu \mathrm{m}$ and $\sigma=40 \mu \mathrm{m}$. Figure $5.1 \mathrm{~b}$ shows the "density function" obtained by simply forming the FFT of the intensity profile measured in the optical transform plane of the same hologram used to obtain Fig. 5.1a. (The peak value of each of the curves is normalized to unity for convenience.) In both graphs, 

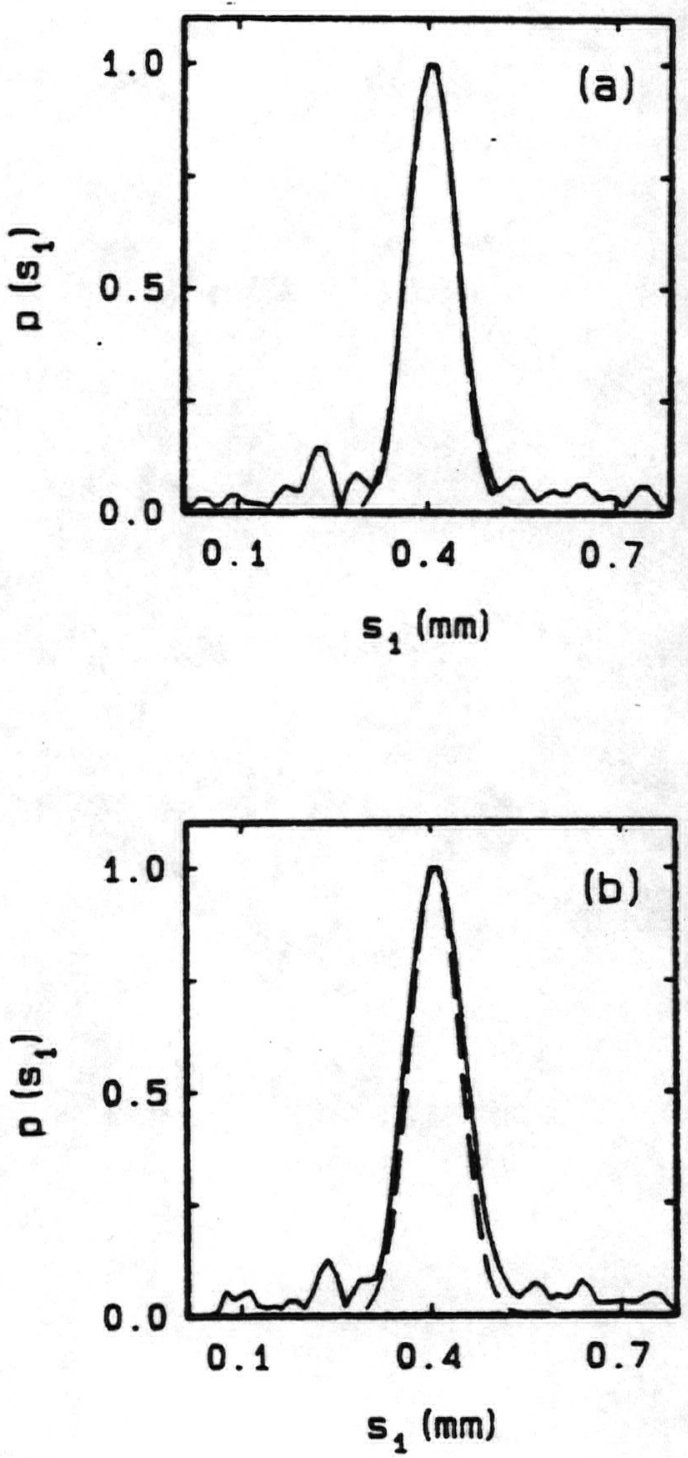

Fig. 5.1. (a) Plot of the computed ( - ) density function and the theoretical (--) density function vs. displacement. This plot was obtained by using the procedure described in Chapter 4. (b) Plot of the approximate (_) density function obtained by computing the FFT of the of the intensity profile in the transform plane of a doubly exposed hologram. (The theoretical (--) density function is also included.) 
the theoretical density function is given by the dotted line. of course, the curve shown in Fig. 5.1b actually depicts the required density function convolved with the FFT of $I_{0}$. Thus, the curve shown in Fig. 5.1b is slightly broader than that in Fig. 5.1a. Since the FFT of $I_{0}$ is relatively narrow, the convolution does not broaden the density function excessively. Hence, if extremely rapid data processing is required, this technique can be employed at the expense of a slight reduction in accuracy.

As discussed in the previous chapter, certain limitations are inherent to the optical-digital method of measuring particle velocity distributions. Specifically, if the flow is "excessively" random, the velocity distribution cannot be measured. As mentioned, the formation of fringes in the transform plane depends on the degree of correlation between the hologram recorded during the first exposure and that recorded during the second exposure. Therefore, if the particle motion between exposures is "excessively" random (i.e., the distribution is characterized by a very wide probability density function), fringes will not be observed in the transform plane and the velocity distribution cannot be determined. However, it should be possible to handle relatively wide distributions by dividing the hologram into sections. Each section may then by analyzed individually, presuming that the distribution represented by each section is sufficiently narrow.

The optical-digital readout system clearly provides a means for rapidly determining the particle velocity distribution from a 
double-exposure hologram. However, the spatial information (i.e.,. the individual particle locations) cannot be obtained with this readout technique. Thus, the rapid acquisition of velocity information is traded against a loss in spatial information. Since the hologram itself contains the displacement information as well as the particle location information, the spatial information can be obtained if necessary. However, using manual readout to obtain the individual particle locations and displacements can be impractical for samples containing many particles. In such applications, automatic readout based on digital image processing techniques (see Section 1.2 .3 and the references contained therein) may be of some value. However, it appears that any of the current digital readout schemes would encounter the same difficulty as the human observer in determining which image pairs are related by translation. Further study is clearly needed in this area.

It should be noted that throughout this thesis, the particles of interest were assumed to be opaque, in which case, the light scattered by the particles may be analyzed in terms of scalar diffraction theory. Of course, many particles encountered in practice are transparent (e.g., aerosol droplets and glass microspheres). Strictly speaking, scattering from transparent objects must be analyzed in terms of electromagnetic theory (see, for example, van de Hulst [1981] and Bohren and Huffman [1983]). However, an approximate solution to the scattering problem is possible when the transparent particles of interest are large 
compared to the wavelength of light. In this case, van de Hulst [1981] maintains that the scattered radiation arises from two distinct phenomena. First, rays striking the particle's surface will be refracted in accordance with Snell's law. (In addition, refracted rays may emerge after several internal reflections.) Second, the boundary of the particle presents a discontinuity to the incident illumination, and hence, diffraction will occur. For a large transparent object, scalar diffraction theory is adequate to describe the diffracted component of the scattered illumination (van de Hulst [1981]). Thus, apart from the refracted component, the scattered field from a transparent object is the same as the scattered field from an opaque particle, assuming both particles are identical in size and shape. In a typical situation (a water droplet in air, for example) the refractive index of the particle is sufficiently different from that of the surrounding medium, and hence, the refracted component of 1 ight will diverge rapidly in comparison to the diffracted component. As a result, the magnitude of the diffracted component is larger than the refracted component over a small region about the forward-scattering direction. To a good approximation, the scattered field in this region is represented by the diffracted component only. Thus our assumption of opaque particles is usually not a serious restriction in practice. It should, however, be noted that holographic images of transparent objects may exhibit structure which is not observed with opaque objects. For example, Fig. 5.2a shows a photograph of images 


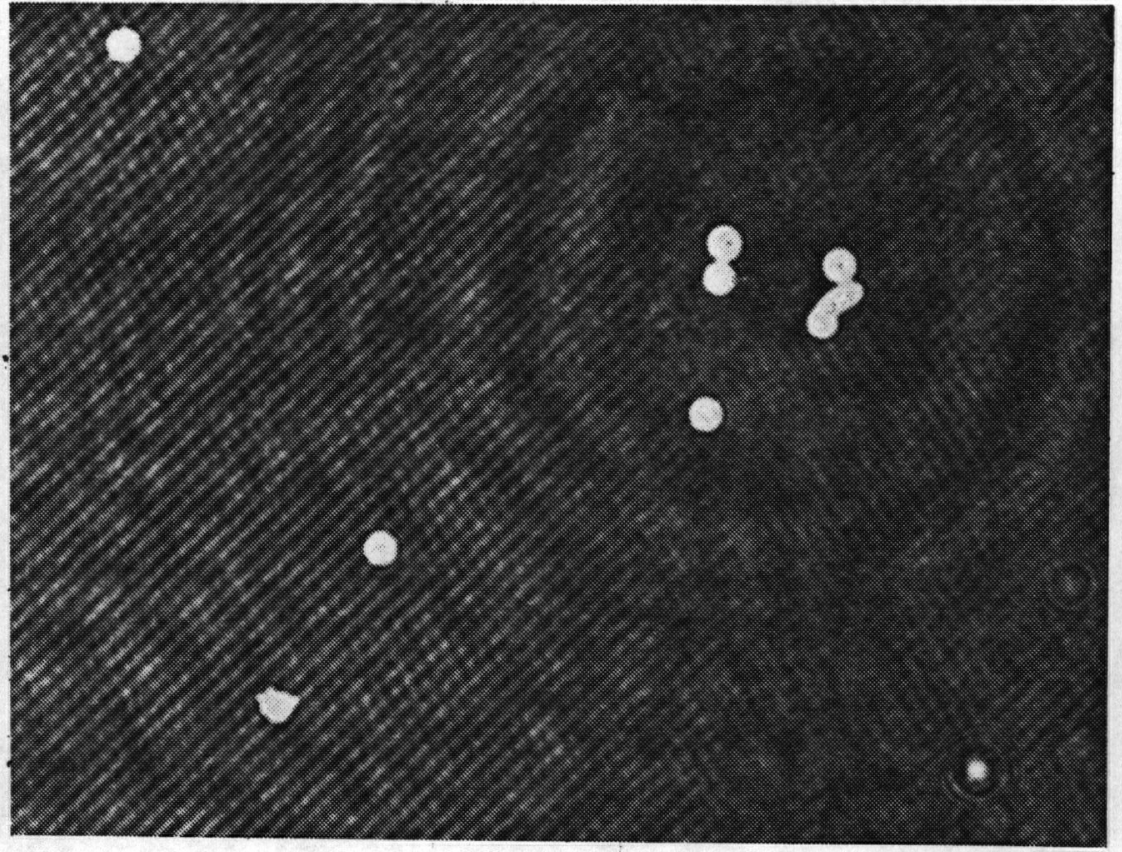

(a)

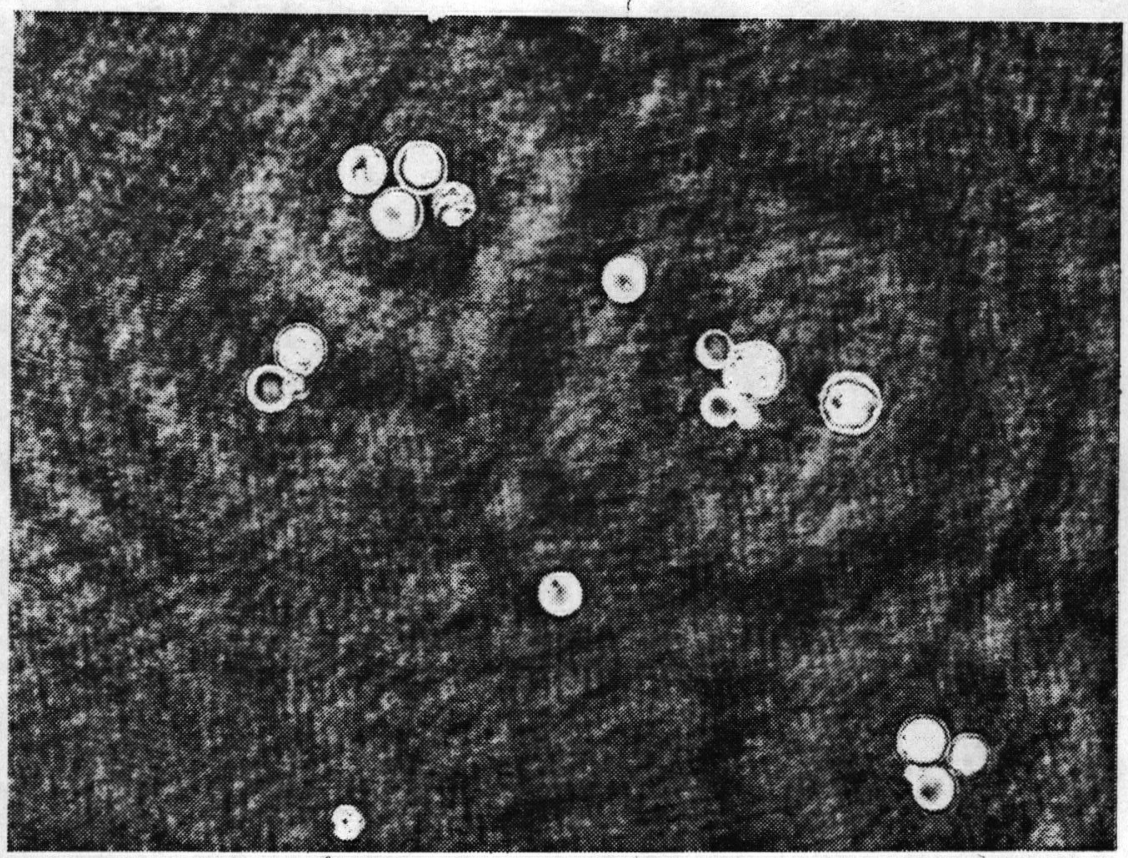

(b)

Fig. 5.2. (a) Real images obtained from a far-field hologram of glass microspheres. (b) Real images obtained from a far-field hologram of glass microballoons. Note that the images of the microballoons exhibit interference fringes. 
obtained from a far-field hologram of $50 \mu \mathrm{m}$ glass microspheres; Fig. $5.2 b$ shows images obtained from a far-field hologram of glass microballoons (ranging in size from approximately $80 \mu \mathrm{m}$ to $150 \mu \mathrm{m}$ ). The images obtained from the microspheres are similar in nature to images obtained from opaque spheres; however, the images of the microballoons exhibit interference fringes. Although the fringes are probably dependent on the diameter and wall thickness of the microballoons, the exact significance of the fringe structure is not presently known. In a similar context, Lomas [1969] has observed interference fringes in the reconstructed image of a far-field hologram of a tapered glass fiber. Although other authors have studied far-field holograms of transparent objects and semitransparent objects (see, for example, Cartwright et al. [1980], Hickling [1969] and Prikryl and Vest [1982]) the characteristics of the resulting images are not well understood in general and further study in this area is required. 


\section{Bibliography}

Abramowitz, M. and I.A. Stegun, eds., Handbook of Mathematical Functions, Dover Publications, New York (1972).

Anderson, W.L., "Particle identification and counting by Fourieroptical pattern recognition," in Applicatons of Optical Fourier Transforms, H. Stark, ed., Academic Press, New York (1982).

Anderson, W.L. and R.E. Beissner, "Counting and classifying small objects by far-field light scattering," Appl. Opt. 10 (7) 15031508 (1971).

Bachalo, W.D. and M.J. Houser, "Phase/Doppler spray analyzer for simultaneous measurements of drop size and velocity distributions," Opt. Eng. 23 (5) 583-590 (1984).

Bexon, R., "Magnification in aerosol sizing by holography," J. Phys. E: Sci Inst. $\underline{6}$ 245-247 (1973).

Bexon, R., J. Gibbs and G.D. Bishop, "Automatic assessment of aerosol holograms," J. Aerosol Sci. I 397-407 (1976).

Boettner, E.A. and B.J. Thompson, "Multiple exposure holography of moving fibrous particulate matter in the respiratory range," Opt. Eng. 12 (2) 56-59 (1973).

Bohren, C.F. and D.R. Huffman, "Absorption and Scattering of Light by Small Particles, Wiley-Interscience, New York (1983).

Born, M. and E. Wolf, Principle of Optics, 5th Edition, Pergamon Press, Oxford (1975).

Bryngdah1, 0. and A. Lohmann, "Nonlinear effects in holography," J. Opt. Soc. Am. $\underline{58}$ (10) 1325-1334 (1968).

Carder, K.L., "Holographic microvelocimeter for use in studying ocean particle dynamics," Opt. Eng. 18 (5) 524-525 (1979).

Cartwright, S.L., Ph.D. Thesis, Aspects of Far-Field Holography, The Institute of Optics, University of Rochester, Rochester, New York (1980).

Cartwright, S.L., P. Dunn and B.J. Thompson, "Particle sizing using far-field holography: new developments," Opt. Eng. $\underline{19}$ (5) 727$733(1980)$.

Collier, R.J., C.B. Burckhardt and L.H. Lin, Optical Holography, Academic, New York (1971). 
Cornillault, J., "Particle size analyzer," Appl. Opt. 11 (2) 265-268 (1972).

Crane, J., P. Dunn, P.H. Malyak and B.J. Thompson, "Particulate velocity and size measurements using holographic and optical processing methods," in Proc. 15th Int. Cong. High Speed Photography and Photonics SPIE vol. 348, 634-642 (1982).

Davenport, W.B., Jr. and W.L. Root, An Introduction to the Theory of Random Signals and Noise, McGraw-Hill Book Company, New York (1958).

Dodge, L.G., "Calibration of the Malvern particle sizer," Appl. Opt. $\underline{23}$ (14) 2415-2419 (1984).

Dudderar, T.D. and P.G. Simpkins, "Laser speckle photography in a fluid medium," Nature 270 45-47 (1977).

Dunn, P. and B.J. Thompson, "Object shape, fringe visibility and resolution in far-field holography," Opt. Eng. 21 (2) 327-332 (1982).

Durst, F., A. Melling and J.H. Whitelaw, Principles and Practice of Laser-Doppler Anemometry, Academic Press, London (1976).

Ewan, B.C.R., "Holographic particle velocity measurement in the Fraunhofer plane," Appl. Opt. 18 (5) 623-626 (1979a).

Ewan, B.C.R., "Particle velocity distribution measurement by holography," Appl. Opt. 18 (18) 3156-3160 (1979b).

Ewan, B.C.R., "Fraunhofer plane analysis of particle field holograms," Appl. Opt. $\underline{19}$ (8) 1368-1372 (1980).

Ewan, B.C.R., J. Swithenbank and C. Sorusbay, "Measurement of transient spray size distributions," Opt. Eng. $\underline{23}$ (5) 620-625 (1984).

Farmer, W.M., "Measurement of particle size, number density and velocity using a laser interferometer," Appl. Opt. 11 (11) 26032612 (1972).

Fourney, M.E., J.H. Matkin and A.P. Waggoner, "Aerosol size and velocity determination via holography," Rev. Sci. Instrum. 40 (2) 205-213 (1969).

Goodman, J.W., Introduction to Fourier Optics, McGraw-Hill, New York (1968). 
Goodman, J.W., "Statistical Properties of Laser Speckle," in Laser Speckle and Related Phenomena, 2nd Edition, J.C. Dainty, ed., Springer-Verlag, Berlin (1984).

Goodman, J.W. and G.R. Knight, "Effects of film nonlinearities on wavefront reconstruction images of diffuse objects," J. Opt. Soc. Am. $\underline{58}$ (9) 1276-2383 (1968).

Grabowski, W., "Measurement of the size and position of aerosol droplets using holography," Optics and Laser Technology 15 (4) 199-205 (1983).

Haussman, G. and W. Lauterborn, "Determination of size and position of fast moving gas bubbles in liquids by digital 3-D image processing of hologram reconstructions," Appl. Opt. 19 (20) 3529-3535 (1980).

Hickling, R., "Holography of liquid droplets," J. Opt. Soc. Am. $\underline{59}$ (10) $1334-1339$ (1969).

Hinsch, K., F. Bader and W. Lauterborn, "The dynamics of cavitation bubble fields studied by double-pulse holography," in Finite Amplitude Wave Effects in Fluids, L. Bjorno, ed. IPC Science and Technology Press, Guilford UK, 240-244 (1974).

Hinsch, K., W. Schipper and D. Mach, "Fringe visibility in speckle velocimetry and the analysis of random flow components," Appl. Opt. $\underline{23}$ (24) 4460-4462 (1984).

Kozma, A. "Analysis of the film non-linearities in hologram recording," Optica Acta 15 (6) 527-551 (1968).

LeBaron, E.I. and E.A. Boettner, "Fiber motion analysis by two-pulse holography," Appl. Opt. 19 (6) 891-894 (1980).

Lomas, G.M., "Interference phenomena in Fraunhofer holograms and in their reconstructions of tapered glass fibers," Appl. Opt. 8 (10) 2037-2041 (1969).

Malyak, P.H. and B. J. Thompson, "Particulate velocity measurement using holographic methods," Proc. SPIE vol. 427, 172-179 (1983).

Menzel R. and F.M. Shofner, "An investigation of Fraunhofer holography for velocimetry applications," Appl. Opt. 9 (9) 20732079 (1970). 
Murakami, T. and M. Ishikawa, "Holographic measurements of velocity distribution of particles accelerated by a shock wave," Proc. 13 th Int. Cong. on High Speed Photography and Photonics, S. Hyodo, Ed., Japan Soc. Precision Eng. SPIE vol. 189, 326-329 (1978).

Payne, P.R., K.L. Carder and R.G. Steward, "Image analysis techniques for holograms of dynamic oceanic particles," Appl. Opt. $\underline{23}$ (2) 204-210 (1984).

Prikryl, I. and C.M. Vest, "Holographic imaging of semitransparent droplets or particles," Appl. Opt. 21 (14) 2541-2547 (1982).

Shofner, F.M., R.W. Menzel, T.H. Gee and R.O. Webb, "Processing holographic velocimetry data," Proc. Int. Congress on Instrumentation in Aerospace Simulation, Farmingdale, New York, 136-144 (1969).

Silverman, B.A., B.J. Thompson and J.H. Ward, "A laser fog disdrometer," J. Appl. Meteorology $\underline{3}$ (6) 792-801 (1964).

Simpkins, P.G. and T.D. Dudderar, "Laser speckle measurement of transient Benard convection," J. Fluid Mech. $\underline{89}$ (4) 665-671 (1978).

Slimani, F., G. Grehan, G. Gouesbet and D. Allano, "Near-field Lorentz-Mie theory and its application to microholography," App1. Opt. 23 (22) 4140-4148 (1984).

Stanton, A.C., H.J. Caulfield and G.W. Steward, "An approach for automated anlaysis of particle holograms," Opt. Eng. $\underline{23}$ (5) 577582 (1984).

Stark, H., "Theory and measurement of the optical Fourier transform," in Applications of Optical Fourier Transforms, H. Stark, ed., Academic, New York (1982).

Stark, H., D. Lee and B. Dimitriadis, "Smoothing of irradiance spectra with finite bandwidth windows with applications to particle size analysis," J. Opt. Soc. Am. $\underline{65}$ (12) 1436-1442 (1975).

Stevenson, W.H., "Principles of laser velocimetry," in Experimental Diagnostics in Gas-Phase Combustion Systems: AIAA Progess in Astronautics and Aeronautics, B.T. Zinn, ed., AIAA 53 307-337 (1977). 
Swithenbank, J., J.M. Beer, D.S. Taylor, D. Abbott and G.C. McCreath, "A laser diagnostic technique for the measurement of droplet and particle size distribution," in Experimental Diagnostics in Gas-Phase Combustion Systems: AIAA Progress in Astronautics and Aeronautics, B.T. Zinn, ed., AIAA 53, 421-447 (1977).

Thompson, B.J., "Holographic particle sizing techniques," J. Phys. E. $\underline{7} 781-788$ (1974).

Thompson, B.J., "Holographic methods of dynamic particulate measurements - current status," in Proc. 15th Int. Cong. High Speed Photography and Photonics SPIE vol. 348, 626-632 (1982).

Thompson, B.J., "Droplet characterization with conventional and holographic imaging techniques," Liquid Particle Size Measurements Techniques, ASTM STP 848 J.M. Tishkoff, R.D. Ingebo, and J.B. Kennedy, Eds., American Society for Testing and Materials, 111-122 (1984).

Thompson, B.J. and P. Dunn, "Advances in far-field holography theory and applications," Proc. SPIE vol. 215, 102-111 (1980).

Thompson, B.J., G.B. Parrent, J.H. Ward and B. Justh, "A readout technique for the laser fog disdrometer," J. Appl. Meterology $\underline{5}$ (3) 343-348 (1966).

Trolinger, J.D., "Particle field holography," Opt. Eng. 14 (5) 383$392(1975)$.

Trolinger, J.D., R.A. Belz and W.M. Farmer, "Holographic techniques for the study of dynamic particle fields," Appl. Opt. $\underline{8}$ (5) 957961 (1969).

Trolinger, J.D., W.M. Farmer and R.A. Belz, "Multiple exposure holography of time varying three-dimensional fields," Appl. Opt. $\underline{7}$ (8) $1640-1641$ (1968).

Tyler, G.A. and B.J. Thompson, "Fraunhofer holography applied to particle size analysis - a reassessment," Optica Acta 23 (9) 685-700 (1976).

Uyemura, T., Y. Yamamoto and K. Tenjinbayashi, "Application of pulse laser holography," Proc. 13th Int. Cong. on High Speed Photography and Photonics, S. Hyodo, Ed., Japan Soc. Precision Eng., SPIE vol. 189, 346-349 (1978).

van de Hulst, H.C., Light Scattering by Small Particles, Dover Publications, New York (1981). 
Vikram, C.S. and M.L. Billet, "Gaussian beam effects in far-field in-line holography," Appl. Opt. 22 (18) 2830-2835 (1983).

Vikram, C.S. and M.L. Billet, "Far-field holography at non-image planes for size analysis of small particles," Appl. Phys. B 33 149-153 (1984a).

Vikram, C.S. and M.L. Billet, "Optimizing image-to-background irradiance ratio in far-field in-line holography," Appl. Opt. 23 (12) 1995-1998 (1984b).

Wertheimer, A.L., H.N. Frock, E.C. Muly, "Light scattering instrumentation for particulate measurements in processes," Proc. SPIE vol. 129, 49-58 (1978).

Woodward, P.M., Probability and Information Theory with Applications to Radar, Pergamon Press, Oxford (1953). 


\section{Appendix A}

\section{The Effects of Intermodulation Terms in the Transform Plane of an In-Line Far-Field Hologram}

In calculating the optical Fourier transform of an in-line far-field hologram (see Chapter 2), it was assumed that the contributions from the intermodulation terms are small, and therefore, may be ignored. The following analysis will demonstrate that these contributions are negligible.

It is assumed that the particle sample consists of $N$ identical objects located in a plane parallel to the hologram plane. As usual, $\xi$ and $\eta$ denote the coordinates in the object plane and $x$ and $y$ denote the coordinates in the hologram plane (see Fig. 2.1a). The location of the $n^{\text {th }}$ particle is denoted by $\xi_{n}$ and $\eta_{n}$. The contribution of the $n^{\text {th }}$ particle to the total complex field distribution in the hologram plane is determined by the HuygensFresnel Principle (see Section 2.2.1) and is given by

$$
\begin{aligned}
\Psi_{H, n}(x, y) & =-\frac{i}{\lambda z} \exp [i k z] \\
& \times \iint_{-\infty}^{+\infty}\left[1-A\left(\xi-\xi_{n}, \eta-\eta_{n}\right)\right] \exp \left\{i \frac{k}{2 z}\left[(x-\xi)^{2}+(y-\eta)^{2}\right]\right\} d \xi d \eta
\end{aligned}
$$

The particle is assumed to be opaque, thus, the function $A(\xi, \eta)$ 
describes the particle's cross-sectional shape. Making the change of variables, $\xi^{\prime}=\xi-\xi_{n}$ and $\eta^{\prime}=\eta-\eta_{n}$, and applying the far-field approximation yields, after a straightforward calculation,

$$
\begin{aligned}
\Psi_{H, n}(x, y) & =\exp [i k z]\left\{1+\frac{i}{\lambda z} \exp \left[i \frac{\pi}{\lambda z}\left(\left(x-\xi_{n}\right)^{2}+\left(y-\eta_{n}\right)^{2}\right)\right]\right. \\
& \left.\times \iint_{-\infty}^{+\infty} A\left(\xi^{\prime}, \eta^{\prime}\right) \exp \left[-i \frac{2 \pi}{\lambda z}\left(\left(x-\xi_{n}\right) \xi^{\prime}+\left(y-\eta_{n}\right) \eta^{\prime}\right)\right] d \xi^{\prime} d \eta^{\prime}\right\} .
\end{aligned}
$$

The complex field distribution produced by a sample containing $N$ identical particles may be obtained by adding together all of the individual contributions, i.e.,

$$
\Psi_{H}(x, y)=\sum_{n=1}^{N} \Psi_{H, n}(x, y)
$$

Taking the squared modulus of the above expression yields the intensity distribution in the hologram plane:

$$
I_{H}(x, y)=\sum_{m=1}^{N} \sum_{n=1}^{N} \Psi_{H, m}^{*}(x, y) \Psi_{H, n}(x, y)
$$

Substituting Equation (A.2) into (A.4) yields

$$
I_{H}(x, y)=1+I_{H}^{(1)}(x, y)+I_{H}^{(2)}(x, y)+I_{H}^{(3)}(x, y),
$$

where 


$$
\begin{aligned}
I_{H}^{(1)}(x, y)= & -\frac{i}{\lambda z} \sum_{n=1}^{N} \exp \left[-i \frac{\pi}{\lambda z}\left(\left(x-\xi_{n}\right)^{2}+\left(y-\eta_{n}\right)^{2}\right)\right] \\
& \times \iint_{-\infty}^{+\infty} A\left(\xi^{\prime}, \eta^{\prime}\right) \exp \left[i \frac{2 \pi}{\lambda z}\left(\left(x-\xi_{n}\right) \xi^{\prime}+\left(y-\eta_{n}\right) \eta^{\prime}\right)\right] d \xi^{\prime} d \eta^{\prime}, \\
I_{H}^{(2)}(x, y)= & \frac{i}{\lambda z} \sum_{n=1}^{N} \exp \left[i \frac{\pi}{\lambda z}\left(\left(x-\xi_{n}\right)^{2}+\left(y-\eta_{n}\right)^{2}\right)\right] \\
& \times \iint_{-\infty}^{+\infty} A\left(\xi, \eta^{\prime}\right) \exp \left[-i \frac{2 \pi}{\lambda z}\left(\left(x-\xi_{n}\right) \xi^{\prime}+\left(y-\eta_{n}\right) \eta^{\prime}\right)\right] d \xi^{\prime} d \eta^{\prime},
\end{aligned}
$$

$$
\begin{aligned}
& I_{H}^{(3)}(x, y)=\frac{i}{\lambda^{2} z^{2}} \sum_{m=1}^{N} \sum_{n=1}^{N} \exp \left[i \frac{\pi}{\lambda z}\left(\left(x-\xi_{n}\right)^{2}-\left(x-\xi_{m}\right)^{2}+\left(y-\eta_{n}\right)^{2}-\left(y-\eta_{m}\right)^{2}\right)\right] \\
& \quad \times \iint_{-\infty}^{+\infty} A(\xi, \eta) A\left(\xi, \eta^{\prime}\right) \\
& \quad \times \exp \left[-i \frac{2 \pi}{\lambda z}\left(\left(x-\xi_{n}\right) \xi-\left(x-\xi_{m}\right) \xi^{\prime}+\left(y-\eta_{n}\right) \eta-\left(y-\eta_{m}\right) \eta^{\prime}\right)\right] d \xi d \xi d \eta d \eta^{\prime}
\end{aligned}
$$

The terms in Equation (A.5d) for which $m=n$ are clearly the selfmodulation terms. The remaining terms (i.e., those corresponding to $m \neq n$ ) are the intermodulation terms; their effect in the transform plane of the hologram will now be examined.

In evaluating the expression for the field distribution in the optical transform plane, it is assumed that the transmittance of the 
hologram is directly proportional to the intensity distribution $I_{H}(x, y)$, i.e.,

$$
t(x, y)=b I_{H}(x, y),
$$

where the constant of proportionality, $b$, is set equal to unity for convenience. The Fourier transform is to be performed optically as shown in Fig. 2.1b; thus, the complex field distribution in the transform plane is given by Equation (2.5) which is rewritten below:

$$
\Psi_{F}(u, v)=\frac{\exp \left[i \frac{k}{2 f}\left(1-\frac{d}{f}\right)\left(u^{2}+v^{2}\right)\right]}{i \lambda f} \iint_{-\infty}^{+\infty} t(x, y) \exp \left[-i \frac{2 \pi}{\lambda f}(u x+v y)\right] d y d x, \text { (A.7) }
$$

As indicated in Fig. 2.1b, $u$ and $v$ are the coordinates in the transform plane, $d$ is the separation between the hologram and the transform lens and $f$ is the focal length of the transform lens. For convenience, it will be assumed that $d=f$; hence, the exponential multiplying the integral in Equation (2.5) assumes a value of unity. To present the analysis in a tractable manner, the contribution of a single intermodulation term to the total field distribution in the transform plane will be examined. From Equations (A.5d) and (A.6), it follows that a typical intermodulation term is given by 


$$
\begin{aligned}
& t_{m n}(x, y)=\frac{1}{\lambda^{2} z^{2}} \exp \left[i \frac{\pi}{\lambda z}\left(\left(x-\xi_{n}\right)^{2}-\left(x-\xi_{m}\right)^{2}+\left(y-\eta_{n}\right)^{2}-\left(y-\eta_{m}\right)^{2}\right)\right] \\
& \times \iint_{-\infty}^{+\infty} A(\xi, \eta) A\left(\xi, \eta^{\prime}\right) \\
& \times \exp \left[-i \frac{2 \pi}{\lambda z}\left(\left(x-\xi_{n}\right) \xi-\left(x-\xi_{m}\right) \xi^{\prime}+\left(y-\eta_{n}\right) \eta-\left(y-\eta_{m}\right) \eta^{\prime}\right)\right] d \xi d \xi d \eta d \eta^{\prime}
\end{aligned}
$$

where $m \neq n$. Hence, its contribution to the total field distribution in the transform plane is obtained by evaluating the following expression:

$$
\Psi_{F, m n}(u, v)=\frac{1}{i \lambda f} \iint_{-\infty}^{+\infty} t_{m n}(x, y) \exp \left[-i \frac{2 \pi}{\lambda f}(u x+v y)\right] d y d x
$$

After a lengthy, but straightforward, calculation Equations (A.8) and (A.9) can be combined to yield

$$
\begin{aligned}
\Psi_{F, m n}(u, v) & =\frac{1}{i \lambda f} \exp \left[i \frac{\pi}{\lambda z}\left(\xi_{m}-\xi_{n}\right)^{2}+\left(\eta_{m}-\eta_{n}\right)^{2}-\frac{2 z \xi_{m} u}{f}-\frac{2 z \eta_{m} v}{f}\right] \\
& \times \iint_{-\infty}^{+\infty} A(\xi, \eta) A\left(\xi-\xi_{m}+\xi_{n}+\frac{z u}{f}, \eta-\eta_{m}+\eta_{n}+\frac{z v}{f}\right) \\
& \times \exp \left[-i \frac{2 \pi}{\lambda z}\left(\left(\xi_{m}-\xi_{n}\right) \xi+\left(\eta_{m}-\eta_{n}\right) \eta\right)\right] d \xi d \eta
\end{aligned}
$$


It should be recognized that $I_{H}{ }^{(1)}(x, y)$ and $I_{H}{ }^{(2)}(x, y)$ (see Equations (A.5b) and (A.5C)) are the image forming terms which, in the transform plane, yield a contribution that corresponds to the total diffraction pattern of the particle sample. Hence, the quantity of interest in the transform plane is the contribution that results from $I_{H}^{(1)}(x, y)$ and $I_{H}^{(2)}(x, y)$. For the total diffraction pattern of an actual particle sample to be equivalent to the Fourier transform of a far-field hologram of the same sample, the contributions from the intermodulation terms must be smaller than the contribution from $I_{H}^{(1)}(x, y)$ and $I_{H}{ }^{(2)}(x, y)$. To compare the respective contributions, it will be convenient to assume a shape for the particles so that the integral in Equation (A.10) may be evaluated in closed form. For convenience, the particles are assumed to have a square cross-sectional shape where the width of a typical particle denoted by $2 a$. Therefore, the object function is given by

$$
A(\xi, \eta)=\operatorname{rect}\left(\frac{\xi}{2 a}\right) \operatorname{rect}\left(\frac{\eta}{2 a}\right),
$$

where $\operatorname{rect}(x)=1$ for $-1 / 2<x<1 / 2$ and is zero otherwise. Substituting the above expression into Equation (A.10) yields 


$$
\begin{aligned}
&\left|\Psi_{F, m n}(u, v)\right|=\frac{1}{\lambda f}\left|\frac{\sin \left[\frac{\pi}{\lambda z} \Delta \xi_{m n}\left(\Delta \xi_{m n}-\frac{z u}{f} \pm 2 a\right)\right]}{\frac{\pi}{\lambda z} \Delta \xi_{m n}}\right| \\
& \times\left|\frac{\sin \left[\frac{\pi}{\lambda z} \Delta \eta_{m n}\left(\Delta \eta_{m n}-\frac{z v}{f} \pm 2 a\right)\right]}{\frac{\pi}{\lambda z} \Delta \eta_{m n}}\right|
\end{aligned}
$$

for $-2 a<\left(\Delta \xi_{m n}-z u / f\right)<2 a$ and $-2 a<\left(\Delta \eta_{m n}-z v / f\right)<2 a$. Outside of this region, $\Psi_{F, m n}$ is identically zero. The quantities $\Delta \xi_{m n}$ and $\Delta \eta_{m n}$ denote the horizontal and vertical separation between the $m^{\text {th }}$ and $n^{\text {th }}$ particles, respectively, i.e., $\Delta \xi_{m n}=\xi_{m}-\xi_{n}$ and $\Delta \eta_{m n}=\eta_{m}-\eta_{n}$. The positive sign is used in the first line of Equation (A.12) when $-2 a<\left(\Delta \xi_{m n}-z u / f\right)<0$; the negative sign is used when $0<\left(\Delta \xi_{m n}-z u / f\right)<2 a$. Similarly, the positive sign is used in the second line of Equation (A.12) when $-2 a<\left(\Delta \eta_{m n}-z u / f\right)<0$; the negative sign is used when $0<\left(\Delta \eta_{m n}-z u / f\right)<2 a$.

Several observations may be made from Equation (A.12) and the inequalities that follow it: (1) The contribution $\Psi_{F, m n}$ from a typical intermodulation term is confined to a square area ( $4 a \times 4 a)$ in the transform plane. If the particles are randomly distributed throughout the sample, then there will be little, if any, overlap between $\Psi_{F, m n}$ and $\Psi_{F, m^{\prime} n^{\prime}}$ (where $m \neq m^{\prime}$ if $n=n^{\prime}$ and $n \neq n^{\prime}$ if $m=m^{\prime}$ ). (2) The magnitude of $\Psi_{F, m n}$ decreases as the separation between the 
$m^{\text {th }}$ and $n^{\text {th }}$ particles increases (i.e., $\left|\Psi_{F, m n}\right|$ decreases as $\Delta \xi_{m n}$ or $\Delta n_{m n}$ increases in magnitude). This result is clearly expected since increasing the particle separation will cause less interaction between the light diffracted by the given particles. (3) As the observation point in the transform plane moves away from the origin, $\Psi_{F, m n}$ will decrease in magnitude. That is, $\Psi_{F, m n}$ decreases as the coordinates $u$ and $v$ increase in magnitude. This may be demonstrated by considering the inequalities following Equation (A.12). For example, the inequality $-2 a<\left(\Delta \xi_{m n}-z u / f\right)<2 a$ must be satisfied whenever $\Psi_{F, m n}$ is nonzero. If $u$ is large, then $\Delta \xi_{m n}$ must be large enough to satisfy the above inequality. However, as stated in (2) above, the magnitude of $\Psi_{F, m n}$ decreases as $\Delta \xi_{m n}$ or $\Delta \eta_{m n}$ increases. Thus, $\left|\Psi_{F, m n}\right|$ must also decrease as $u$ increases.

The dependence of $\left|\Psi_{F, m n}\right|$ on $u$ and $v$ may be written in an explicit form when the observation point in the transform plane is a large distance from the origin (i.e., the distance between the origin and the observaton point is much greater than the particle width). In this case, the inequalities following Equation (A.12) indicate that

$$
\Delta \xi_{m n} \approx \frac{z u}{f}
$$

and

$$
\Delta \eta_{m n} \approx \frac{z v}{f}
$$


Substituting the above results into the denominator of Equation (A.12) yields

$$
\begin{aligned}
&\left|\Psi_{F, m n}(u, v)\right|=\frac{1}{\lambda f}\left|\frac{\sin \left[\frac{\pi}{\lambda z} \Delta \xi_{m n}\left(\Delta \xi_{m n}-\frac{z u}{f} \pm 2 a\right)\right]}{\frac{\pi}{\lambda f} u}\right| \\
& \times\left|\frac{\sin \left[\frac{\pi}{\lambda z} \Delta \eta_{m n}\left(\Delta \eta_{m n}-\frac{z v}{f} \pm 2 a\right)\right]}{\frac{\pi}{\lambda f} v}\right|
\end{aligned}
$$

for $-2 a<\left(\Delta \xi_{m n}-z u / f\right)<2 a$ and $-2 a<\left(\Delta \eta_{m n}-z v / f\right)<2 a$. Outside of this region, the quantity $\Psi_{F, m n}$ is identically zero.

It should be recalled from Chapter 2 that the contribution from the image forming terms (denoted by $\Psi_{F, I}$ in the present discussion) is random since the individual particle locations are random. As a result, it is of interest to consider the expected value of $\Psi_{F, I^{*}}$ An expression for $\left\langle\Psi_{F, I} \Psi_{F, I}{ }^{*}\right\rangle$ was obtained in Section 2.2.2; in order to compare this quantity to $\left|\Psi_{F, m n}\right|$, we define the quantity $\left|\Psi_{F, I}\right|_{r m s}$ where $\left|\Psi_{F, I}\right|_{r m s}=\left[\left\langle\Psi_{F, I} \Psi_{F, I}{ }^{*}\right]^{1 / 2}\right.$. Combining this definition with the results of Section $\mathbf{2 . 2 . 2}$ yields

$$
\left|\Psi_{F, I}(u, v)\right|_{r m s}=\frac{2}{\lambda f} N^{1 / 2}\left|\tilde{A}\left(\frac{u}{\lambda f}, \frac{v}{\lambda f}\right) \cos \left(\frac{\pi z \rho^{2}}{\lambda f^{2}}\right)\right|
$$

The above expression then reduces to 


$$
\left|\Psi_{F I}(u, v)\right|_{r m s}=\frac{2}{\lambda f} N^{1 / 2}\left|4 a^{2} \operatorname{sinc}\left(\frac{2 u a}{\lambda f}\right) \operatorname{sinc}\left(\frac{2 v a}{\lambda f}\right) \cos \left(\frac{\pi z \rho^{2}}{\lambda f^{2}}\right)\right|
$$

for the object function given in Equation (A.11).

The contribution from the image forming terms will now be compared to the contribution from the intermodulation terms. At the origin of the transform plane, the expression for $\left|\Psi_{F, I}\right|_{r m s}$ becomes

$$
\left|\Psi_{F I}(0,0)\right|_{r m s}=\frac{2}{\lambda f} N^{1 / 2} 4 a^{2}
$$

Also, the inequalities following Equation (A.12) reduce to $-2 a<\Delta \xi_{m n}<2 a$ and $-2 a<\Delta \eta_{m n}<2 a$ when $u=v=0$. Hence, $\Delta \xi_{m n}$ and $\Delta \eta_{m n}$ are small and the approximation $\sin x \approx x$ may be applied in the numerator of Equation (A.12). As a result, the expression for $\left|\Psi_{F, m n}\right|$ evaluated at the origin is

$$
\left|\Psi_{F, m n}(0,0)\right|=\frac{1}{\lambda f}\left(\Delta \xi_{m n} \pm 2 a\right)\left(\Delta \eta_{m n} \pm 2 a\right)
$$

The maximum value of the above expression occurs when $\Delta \xi_{m n}=\Delta \eta_{m n}=0$ i.e.,

$$
\left|\Psi_{F, m n}(0,0)\right|=\frac{1}{\lambda f} 4 a^{2}
$$

Thus, for a typical sample containing 10,000 particles,

$$
\left|\Psi_{F, I}(0,0)\right|_{r m s} \gg\left|\Psi_{F, m n}(0,0)\right|
$$


Additionally, it is observed from Equations (A.15) and (A.17) that both $\left|\Psi_{F, m n}\right|$ and $\left|\Psi_{F, I}\right|$ are decreasing functions of $u$ and $v$ since each of these equations contain terms proportional to $u$ and $v$ in their respective denominators. Therefore, the above inequality remains valid at points away from the origin of the transfrom plane (except when $\left|\Psi_{F, I}\right|_{r m s}$ approaches zero). Hence, it is permissible to neglect the intermodulation terms in computing the optical Fourier transform of an in-line hologram. 


\section{Appendix B}

\section{Multiple-Exposure Holography}

Multiple-exposure holography may, of course, be useful in measuring particle displacements and velocities (see also Crane etal. [1982] and Malyak and Thompson [1983]). To illustrate, a fourexposure hologram of $80 \mu \mathrm{m}$ pollen particles was recorded and the reconstructed image from the resulting hologram is shown in Fig. B.1. (Particle motion was simulated by placing the pollen on a glass plate and translating the plate between exposures.) It should be noted that if the particles of interest are non-spherical, this method can provide information on the rate of particle rotation in addition to velocity and size information.

The optical Fourier transform of a multiple-exposure hologram will clearly display velocity-dependent fringes; however, the fringes observed will be sharper than those obtained from a doubleexposure hologram. The fringes are sharper since multiple beam interference fringes are sharper than dual beam interference fringes. This is illustrated in Fig. B.2. Figure B.2a shows the transform of a double-exposure hologram for which all particles had the same displacement between exposures. A similar hologram, using 4 exposures, was also recorded and its transform is shown in Fig. B.2b. The use of digital processing to evaluate the resulting 


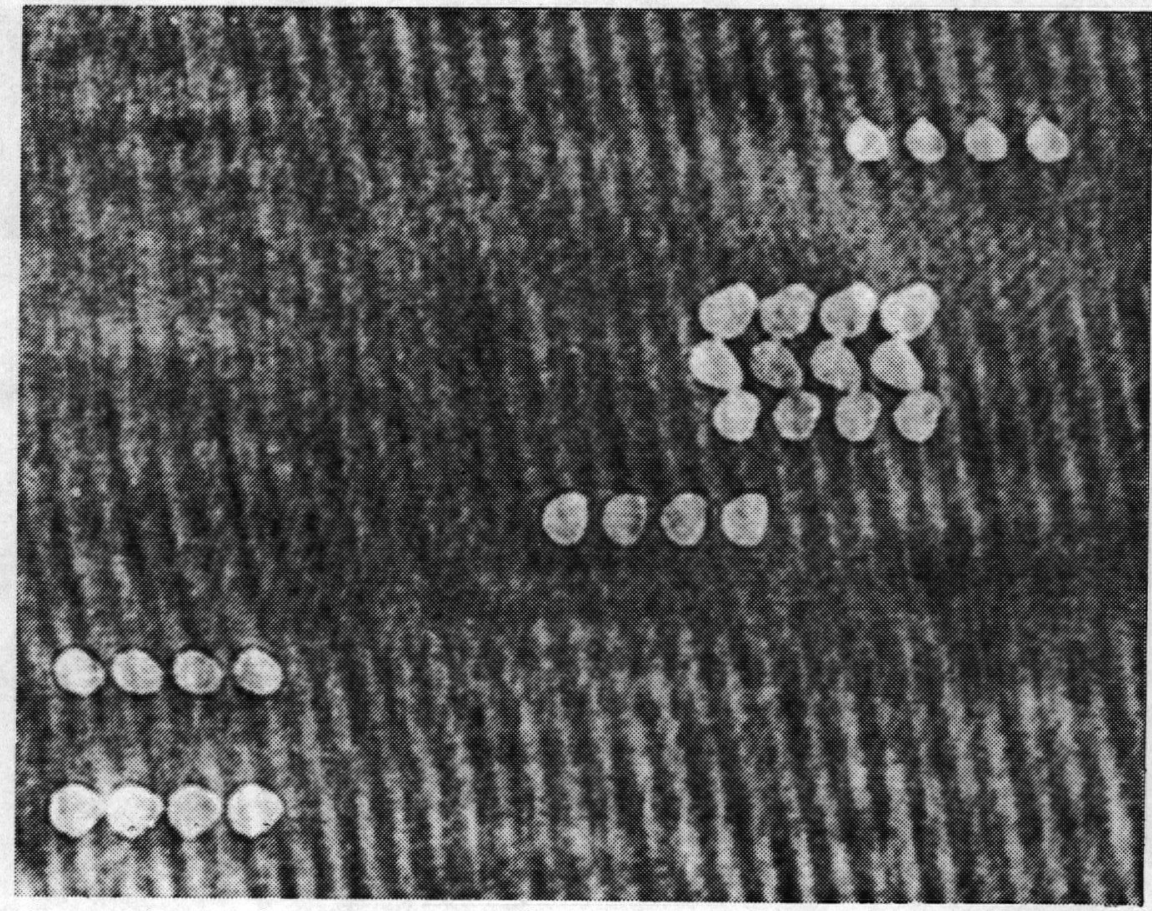

Fig. B.1. Reconstructed images obtained from a four-exposure hologram of $80 \mu \mathrm{m}$ pollen particles. 


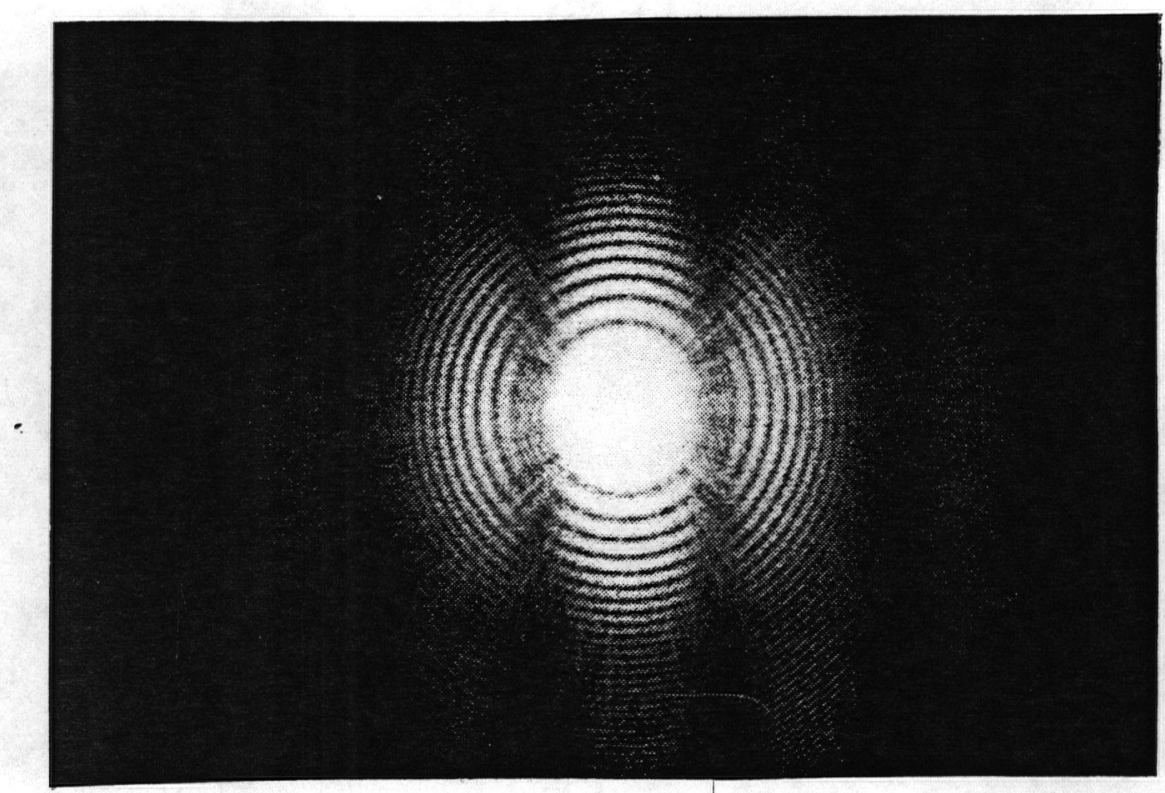

(a)

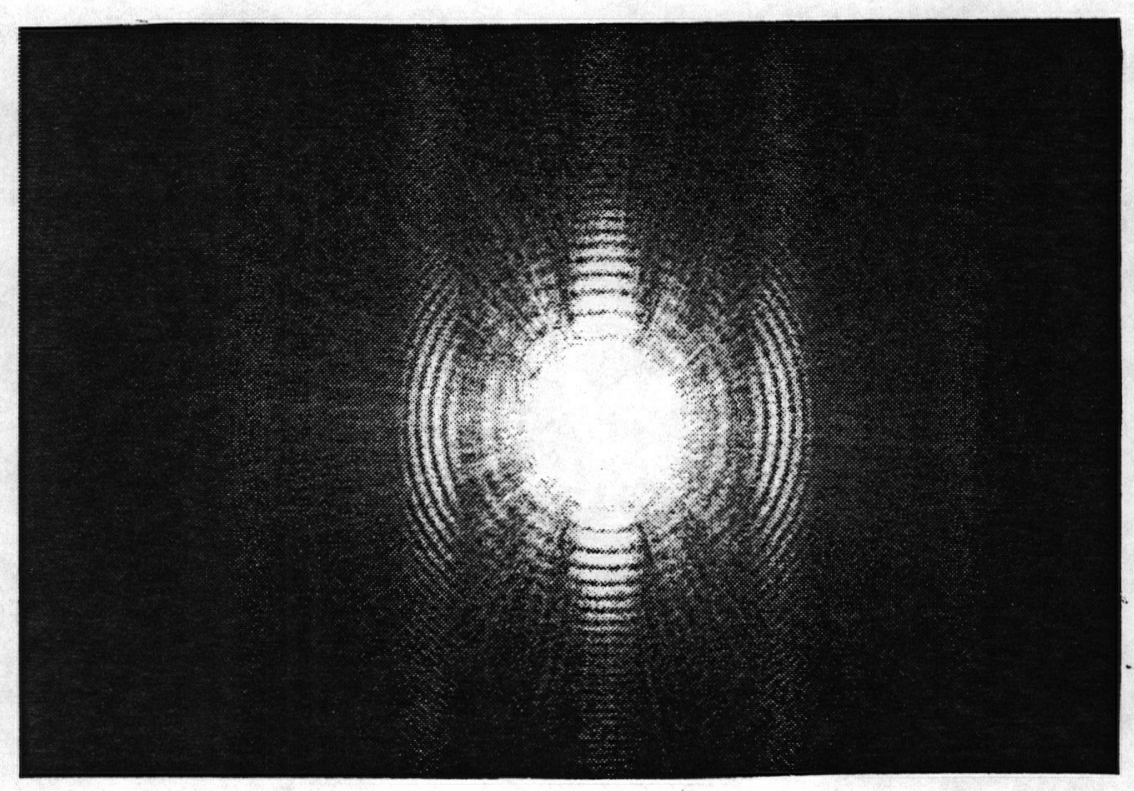

(b)

Fig. B.2. (a) Optical Fourier transform of a double-exposure hologram. (b) Optical Fourier transform of a four-exposure hologram. 
fringe pattern has not been investigated. However, the sharper fringes resulting from multiple-exposure holography appear to be of greater advantage when the transform is to be interpreted visually rather than electronically. 


\section{Appendix C}

\section{Object Motion During Exposure}

In recording a hologram of a dynamic sample, the particles of interest will move a certain distance during the time of exposure. An excessive amount of particle motion will yield a badly degraded image, or no image at all, when the resulting hologram is played back. Thus it is necessary to establish a criterion for the amount of particle motion allowed during exposure. (Thompson [1974] has suggested a tenth of a diameter as a useful criterion.)

The amount of particle motion which can be tolerated is readily established experimentally (see also Crane etal. [1982]). If a series of holograms is recorded, each with a different amount of object translation during exposure, the resulting images may be examined, and hence, an upper limit on particle motion can be determined. Such a series of holgrams was recorded using the inline geometry shown in Fig. 2.1a. The particle sample consisted of $50 \mu \mathrm{m}$ glass microspheres supported on a glass plate; the glass plate was moving at a constant speed for the duration of the exposure. Figures C.la, C.Ib, C.2a and C.2b show the reconstructed images for particle motion of $d / 10, d / 5, d / 2$, and $d$, respectively, where $d$ denotes the particle diameter.

The results shown indicate that a translation of $d$ during 


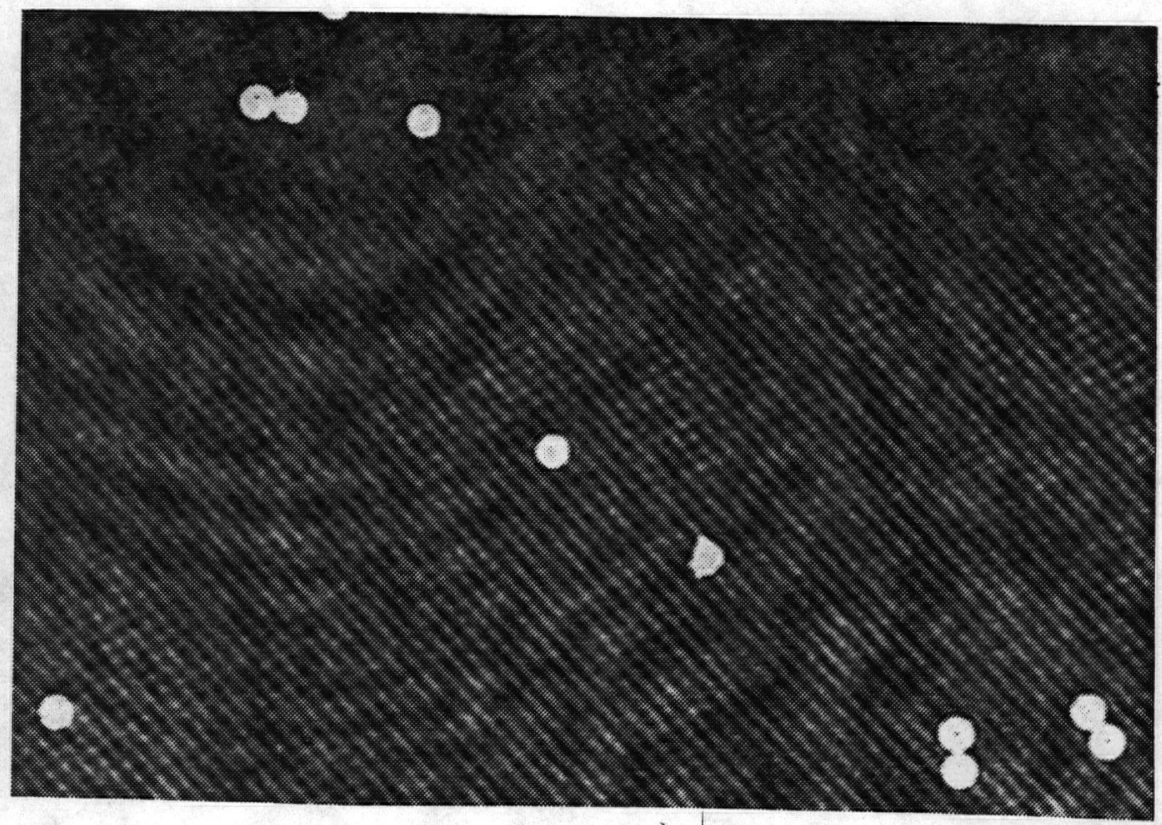

(a)

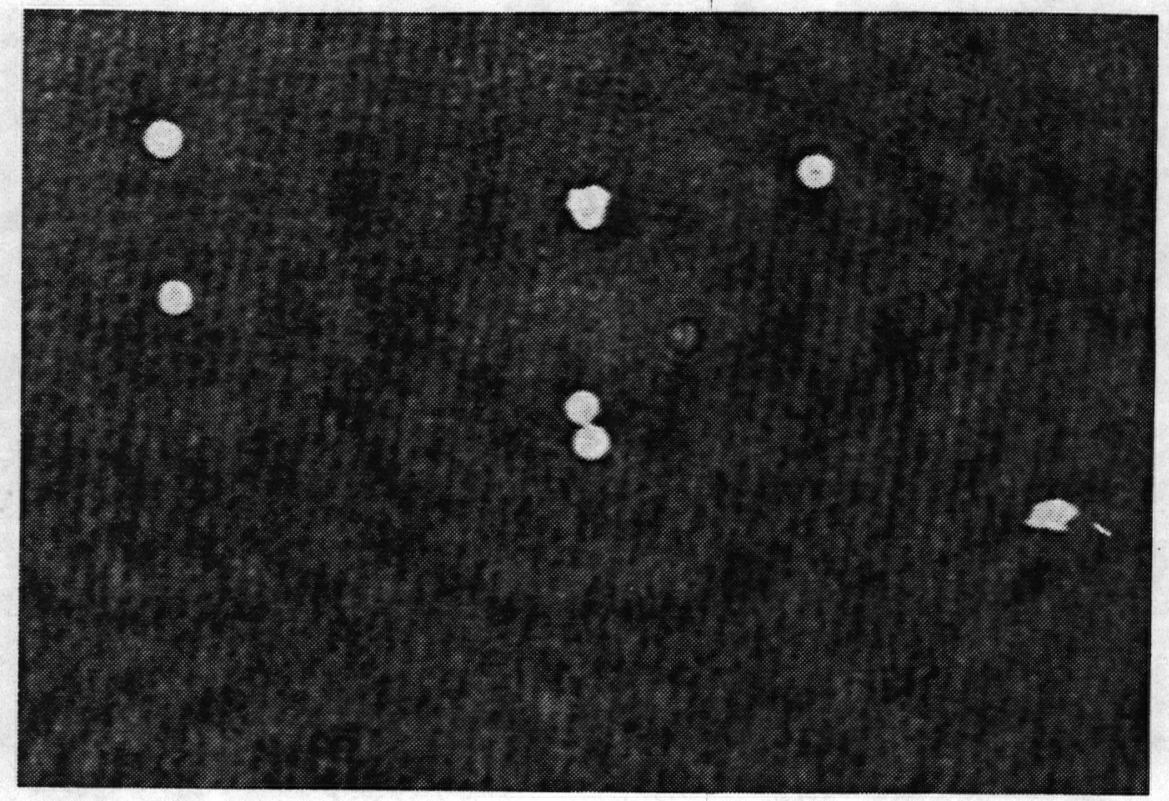

(b)

Fig. C.1. (a) Reconstructed images obtained from a hologram of $50 \mu \mathrm{m}$ glass microspheres. The particles were translated horizontally by $d / 10$ during exposure. (b) Same as (a) except the particle translation was $d / 5$. 


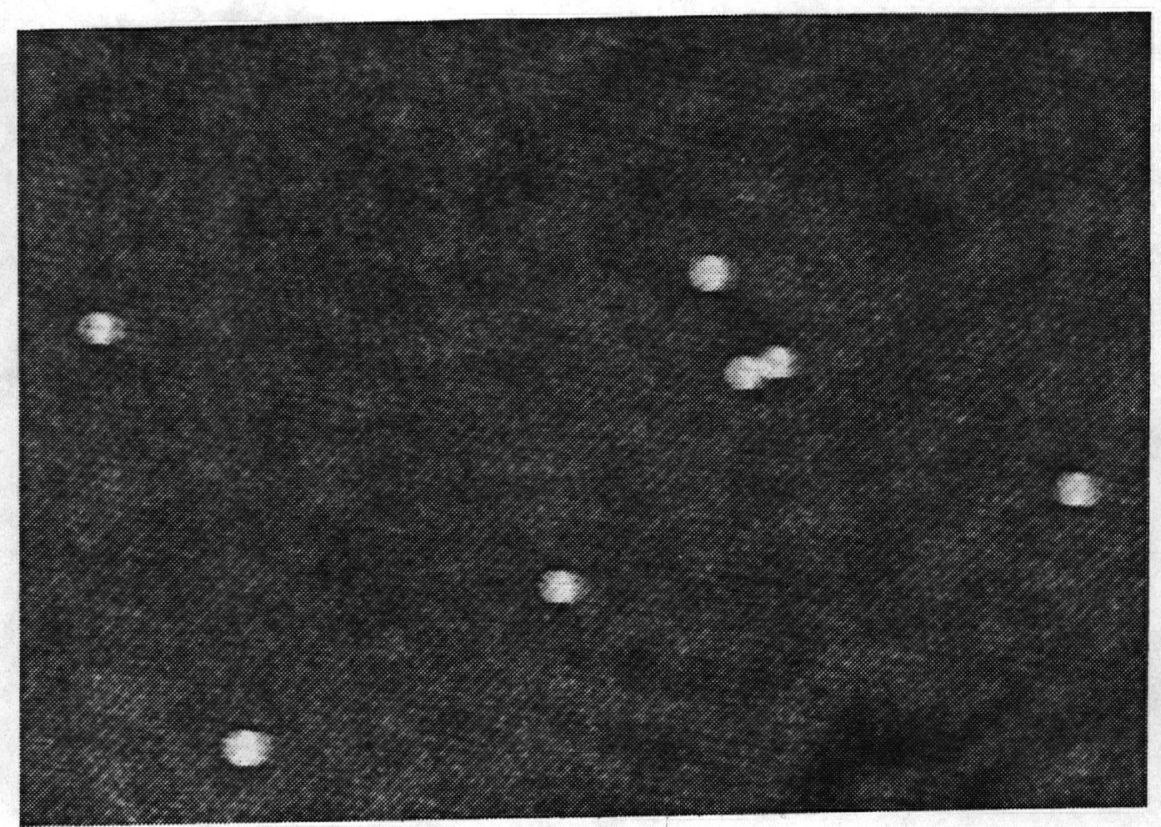

(a)

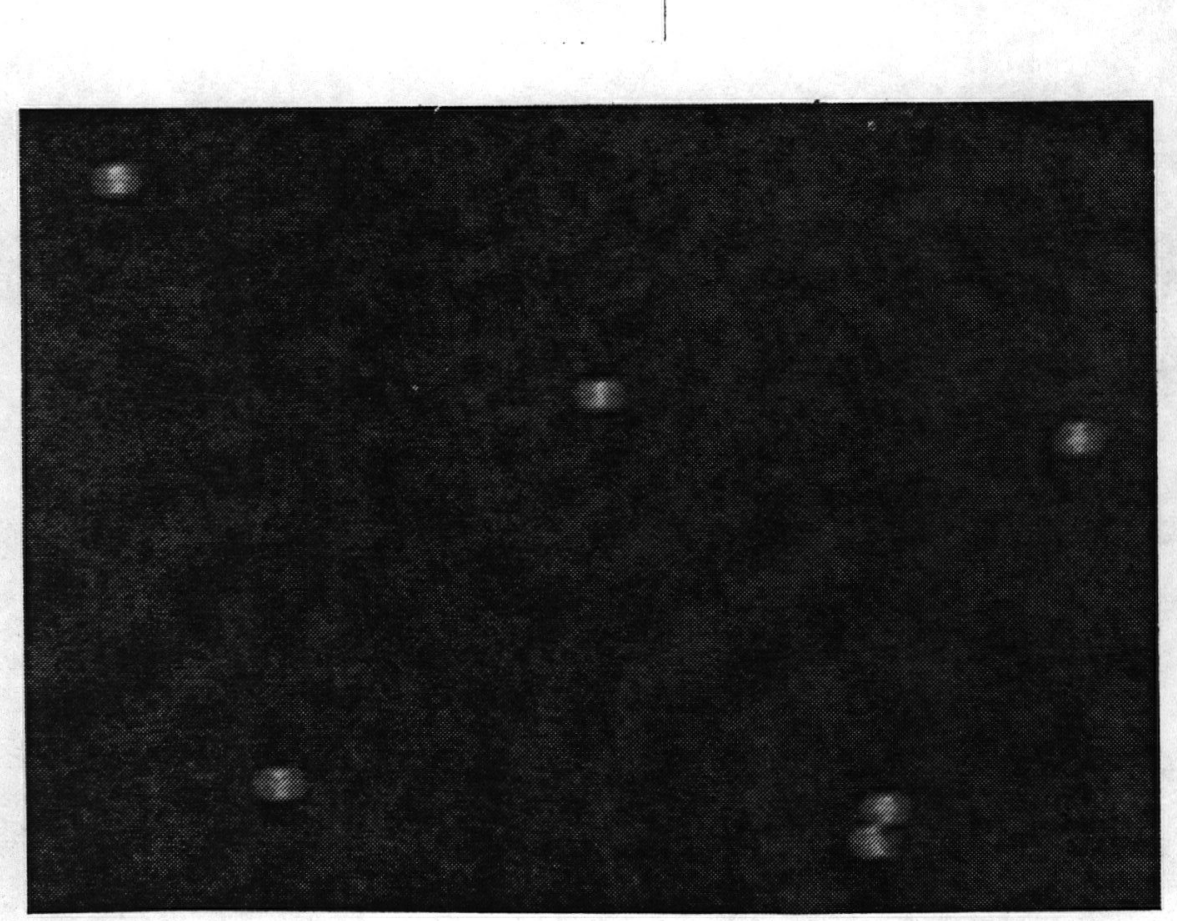

(b)

Fig. C.2. Same as Fig. C.1 except the particle translation was (a) $d / 2$ and (b) $d$. 
exposure still yields a reconstructed image. However, edge definition is degraded. Also, the particle shape is obscured; the reconstructed images are elongated in the direction of particle motion. As the object motion increases, the reconstructed image becomes progessively worse until no image is obtained. Therefore, for the particles considered here, a translation of $d / 10$ during exposure effectively freezes the object motion in time. However, an exposure time allowing particle translation of the order of one diameter may be acceptable if a somewhat degraded image can be tolerated. 